\title{
MADCapHorse
}

A Revised Checklist and Nomenclatural Guide to the Vascular Plants of the Niagara Frontier Region:

Flora of the Niagara Frontier Region, Third Supplement

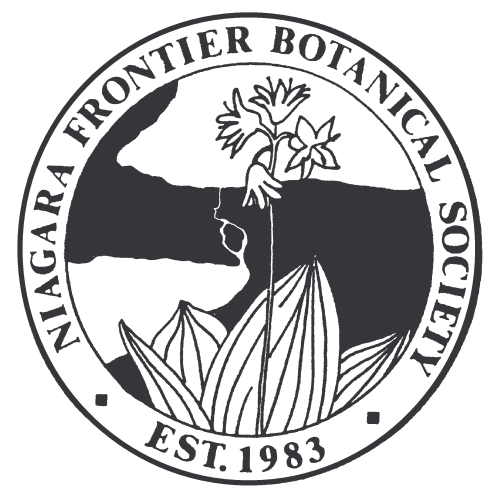

P. M. ECKEL

Research Scientist, Missouri Botanical Garden, St. Louis, Missouri Research Associate, Buffalo Museum of Science, Buffalo, New York

Published by

The Niagara Frontier Botanical Society

with the major support of

Kenneth W. Brandes in remembrance of Barbara A. Brandes

The Buffalo Audubon Society

The Buffalo Museum of Science

The Central/Western New York Chapter of the Nature Conservancy

The New York Flora Association

The Nature Sanctuary Society of Western New York

BULLETIN OF THE BUFFALO SOCIETY OF NATURAL SCIENCES

VOLUME 16 (SUPPLEMENT 3) 2005 


\section{The Buffalo Society of Natural Sciences}

The Buffalo Museum of Science

1020 Humboldt Parkway

Buffalo, New York 14211-1293 USA

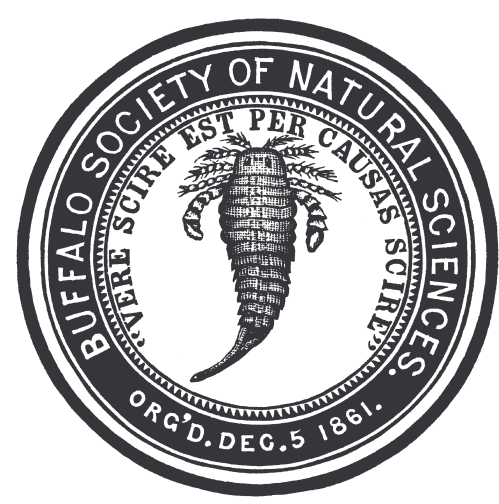

This work is dedicated to Richard H. Zander.

The cover photograph of Clintonia borealis is by Lincoln Nutting, and is used with permission.

MADCAPHORSE, A Revised Checklist of the Vascular Plants of the Niagara Frontier Region: Flora of the Niagara Frontier Region, Third Supplement Bulletin of the Buffalo Society of Natural Sciences, Vol. 16(3).

ISSN 0096-4131; ISBN 0944032-57-5.

Copyright (C) 2005 by Patricia M. Eckel. All rights reserved. 


\section{MADCapHorse}

\section{INTRODUCTION}

The mnemonic device MADCapHorse is a handy guide used by field botanists. It aides in identifying tree and shrub species with opposite leaves (Maple, Ash, Dogwood, CAPrifoliaceae and HORSEchestnut). It is not fully discriminative as many of the herbaceous families and genera presented here also have opposite leaves. Most conspicuously, all Mint species (family Lamiaceae) have opposite, simple (but not compound) leaves as do several important genera in the Rubiaceae.

This is a list of the species of vascular plants known to occur in the Niagara Frontier region. This is traditionally a circular area with the radius of 50 miles having its central point located in the City of Buffalo, New York, specifically "determined from the Telephone Building" (Zenkert 1934). There, the waters of Lake Erie gather themselves into the head of the Niagara River, a strait connecting Erie and Lake Ontario. The Niagara Frontier region comprises an area of about 7,850 square miles.

The Niagara River has probably formed a critical link in the migration of flora and fauna between lakes Erie and Ontario, and generally the Great Lakes system as a whole. Species have migrated through the system through incremental advancement or through long-distance dispersal, for instance, as seeds in the bellies of birds. Other pathways include human-mediated movement along the various canals that bypass the great obstruction of the cataracts at Niagara Falls as well as canals up- and downstream from our area. One curious feature of plant migration regionally is that although the water flows from west to east from Lake Superior to the St. Lawrence River, the migration of species has tended to be from east to west, as with shipping in the Rideau Canal that connects Kingston, Ontario and the Ottawa river to points upstream.

Other sources of regional plant introduction derive from western centers and may increase as the growing season is extended with warming annual temperatures. Extension of the growing season in recent times has also contributed to the firm establishment of species that were once considered ephemeral, not persisting beyond their first winter, or unable to reproduce through absence of, for example, a pollinator. Areas with shallow soils over limestone in the lowlands associated with the lakes are particularly likely to support introduced species and enable their populations to flourish. Introductions once commonly arriving along railroad vectors now seem to favor the salty corridors beside ever-expanding highway systems. Always, wherever crops are grown, associated weeds become established on field margins. With the realestate explosion of the past two decades exotic species associated with nurseries and imported soil are increasingly established. Hybridization events or artifacts may contribute taxa, for instance, in the spread of Lonicera morrowii $\times$ tatarica.

Happily, not all newly discovered taxa are foreign. Several recent discoveries of indigenous taxa have been made through vigorous exploration by field botanists who specialize 
in recording the localities of rare plants. A strong amateur interest has also produced many new discoveries of native and exotic species.

George W. Clinton, Superior Court Judge and first President of the Buffalo Society of Natural Sciences published, in 1863, the first checklist of the "Plants of Buffalo and its Vicinity," which also included references to collections from Chautauqua County, Rochester in Monroe County, and Portage in Livingston County. David F. Day, in 1882, determined the present areal definition of the flora in his new checklist. In spite of its artificiality, this range circumscription is superior to publications that restrict their field of study to political limits and treat only New York or Ontario. The floral region includes one of the most phytogeographically interesting areas in the Province of Ontario: the Niagara Peninsula (Regional Municipality of Niagara), as well as all or most of the eight westernmost counties of New York State.

The extensive early history of collecting and reporting in the Niagara region is provided by Zenkert (1934). It is well recognized that there has been a surge of botanical interest in the region beginning in the early 1980s, especially with the establishment of the Niagara Frontier Botanical Society in 1983 in Buffalo, New York. Many floristic reports and articles may be found in the society's newsletter Clintonia. The present list is an attempt to summarize all reports, to adjust the nomenclature of Zander and Pierce's (1979) list to the nomenclature of the standard checklist of New York State plants by Richard Mitchell and Gordon Tucker (1997), and to give local collectors a guide to what is already known and, by extension, what is yet to be discovered in the ever-changing flora of our region.

The plant names of the Checklist are primarily based on specimens curated in the Clinton Herbarium (BUF) of the Buffalo Museum of Science. The online Web database of the New York Flora Association indicates that the Association's distribution reports are based on specimens and such reports are therefore cited here. Where a report occurs in literature only, the bibliographic reference is given.

Botanical activity leading to additions to the Checklist has been one of the central services that the Buffalo Society of Natural Sciences has provided the people of the Niagara Frontier Region, in New York and Ontario alike, since the time of the Civil War. The Checklist, in its 1863, 1882-83, 1934, 1975, 1979, and present 2005 versions, has always guided and stimulated botanical activity in the Niagara Region, and it is hoped this update will be no exception.

P. M. Eckel, Research Scientist, Missouri Botanical Garden, St. Louis, Missouri 


\section{ABBREVIATIONS AND CODES}

To streamline citations, certain data flags were provided. Genus names are in BOLDFACE CAPITALS. Species names in boldface are accepted as well-established members of the flora, though some may be extirpated at present. Species names with an asterisk $(*)$ are those thought to be alien invaders, adventives or introduced. Names preceded by an equal sign (=) are synonyms of the boldfaced name above them, being names previously used by students of the flora but now superceded by names considered correct according to the formal botanical nomenclatural rules, or are much-used but mistaken identifications and are identified as such. Names in Roman lightface are cross-indexed synonyms, alphabetized for ease in finding them.

\section{RARE, THREATENED AND ENDANGERED SPECIES}

The list of rare, threatened and endangered species changes periodically with research results. The New York State Natural Heritage Program may be consulted as to the most recent official designations.

\section{EXTIRPATED SPECIES}

Many uncommon species are under pressure from human development and other changing conditions. Some have not been located for decades while others are only suspected of being lost to the flora. Though one cannot prove absence from the flora, certain species listed here are doubtless entirely gone from our flora. None is extinct, however, as they are present in other areas.

\section{ADVENTIVES AND GARDEN WEEDS}

To qualify as a member of a flora it is generally necessary that the species establish persistent populations. Locally, a minimum requirement for this includes survival of low winter temperatures, shortness of growing season, and competition with impinging species populations. The chance seed that sprouts but which would have died out for various reasons does not qualify for membership, even though the plant may have made its way into a herbarium collection. Most of our horticultural and agricultural species are not persistent and hence are not considered members of the flora.

It is remarkable, then, the large number of taxa that have been recently reported as "new" to our flora. Undoubtedly populations of species once eliminated by the rigors of the regional environment are now persistent for a variety of reasons, such as the extension of the growing season, the amelioration of the lowest of winter temperatures and their duration, the spread of pollination vectors (insects), a possible increase in the concentration of carbon dioxide in the atmosphere, and other factors enhancing the vigor of certain species.

The present author can vouch for several garden species that appear to have spread, especially in disturbed areas. Catalpa (Catalpa speciosa), with its magnificent, orchid-like 
flowers, is not only established, but looks likely to become as vigorous a weed as Box Elder (Acer negundo) or White Mulberry (Morus alba). This is also true for Sycamore Maple (Acer pseudoplatanus), which springs up along the curbs of the streets of Buffalo.

For more information on new discoveries, see the list of "Names New to the Flora Since the Second Supplement" given on page 151 as Appendix 1.

\section{ONLINE RESOURCES}

The World Wide Web has many features that allow the updating of information in this checklist, and provide images and additional information not available in print.

An early, online version of this publication, with some images, is at Res Botanica: http: //www.mobot.org/plantscience/ResBot/flor/WNY-Niag/WNYCheck.htm

The New York Flora Association provides a plant atlas for the state: http: //www.nyflora.org/atlas/atlas.htm

The Niagara Frontier Botanical Society is a regional resource promoting study and appreciation of field botany:

http: //www.acsu.buffalo.edu/ insrisg/botany/

The Buffalo Audubon Society supports and popularizes nature conservation and study: http: //www.buffaloaudubon.com/

The Buffalo Museum of Science houses the Clinton Herbarium and has exhibits on botany. This is also an alternative name for the Buffalo Society of Natural Sciences:

http: //www.sciencebuff.org/welcome.php

The Central/Western New York Chapter of the Nature Conservancy helps preserve the diversity of life in the region through its well-known stewardship activities:

http: //nature.org/

The New York State Natural Heritage Program facilitates conservation of New York's rare animals, rare plants, and significant ecosystems:

http://www.dec.state.ny.us/website/dfwmr/heritage/

The Nature Sanctuary Society of Western New York has for many decades maintained sanctuaries for the preservation of regional plant and animal life:

http: //www.nfwhc.org/naturesanctuary.htm

Michael J. Oldham has created an online database for the "Natural Heritage Resources of Ontario: Rare Vascular Plants" (see bibliography) with periodic updates:

http://www.mnr.gov.on.ca/MNR/nhic/species/lists/rarevascular.pdf 


\section{ACKNOWLEDGEMENTS}

My greatest debt of gratitude is to Richard Zander, who provided essential help with problems in taxonomy throughout the course of this effort. I thank James Battaglia for convincing forward-looking financial supporters, listed on the title page and below, of the need for publication of the printed version of the Checklist. The fine cover photograph was taken by Lincoln Nutting and is used with appreciation. Michael Smith, former Director of the Buffalo Museum of Science, graciously provided computer access to make the first version of this checklist public. John Grehan, Director of Science and Collections, and Kathryn Leacock, Curator of Collections, of the Buffalo Museum of Science, kindly provided facilities at the Museum for completing this checklist. I appreciate the facility support of the Missouri Botanical Garden, Robert E. Magill, Director of Research. Carol Sweeney, of Niagara University, James Battaglia, and Joanne Schlegel provided useful criticism and helpful format guidance in the preparation of this work. Steven Clemants provided access to information on the rare vascular plants of Ontario. Grateful acknowledgement is also made to Irene Wingerter and Steven Zenger of Partner's Press, who provided crucial last minute assistance to the author.

Special appreciation is also extended to the following persons for their support in

publishing this Revised Checklist and Nomenclatural Guide to the Vascular Plants of the Niagara Frontier Region:

Dr. John Hodson, Joanne Schlegel (in memory of Blake Reeves), Michael Siuta, Dr. Carol Sweeney, Dr. Alfred Stein, and Mary Alice Tocke. 


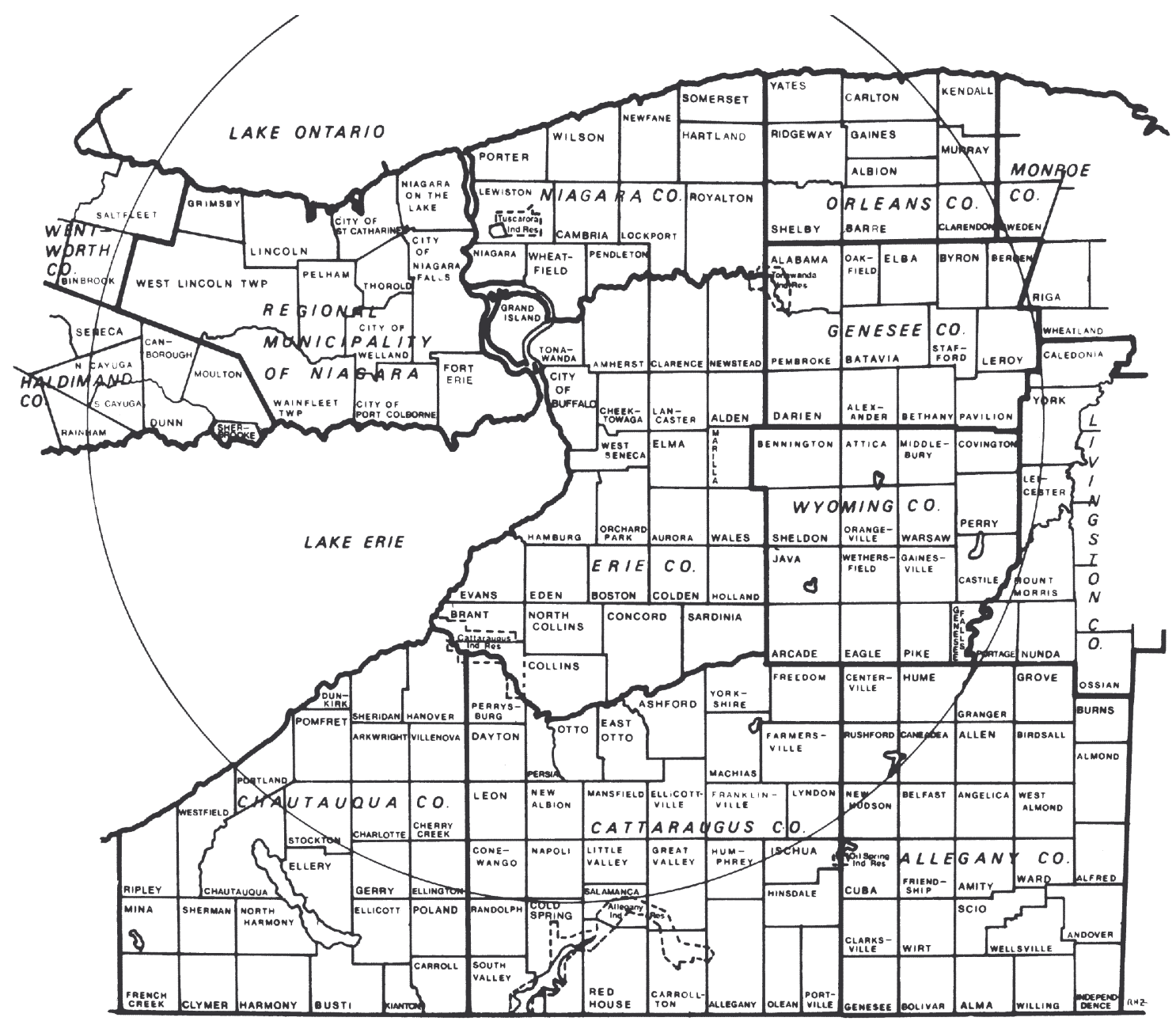

Major political divisions in western New York State and adjoining Canada. County subdivisions are "towns" (equivalent to "townships" in other states) unless otherwise noted. The circle gives the area of the Flora, the area within a fifty-mile radius around Buffalo. 


\section{THE CHECKLIST}

ABIES Mill. (Pinaceae)

Abies alba Michx. = Picea glauca (Moench) Voss

A. balsamea (L.) Mill., Balsam Fir, Canada Balsam, Blisters

Abies canadensis Michx. $=$ Tsuga canadensis (L.) Carr.

Abies nigra Poir. of Clinton's Journal (1864) = Picea mariana (Mill.) BSP

\section{ABUTILON Mill. (Malvaceae)}

Abutilon abutilon (L.) Rusby $=$ Abutilon theophrasti Medik.

*A. theophrasti Medik., Velvet-leaf, Butter-print, Pie-marker, Indian Mallow

$=$ A. avicennae Gaertn.

= A. abutilon (L.) Rusby

$=$ Sida abutilon L.

Abutilon avicennae Gaertn. $=$ Abutilon theophrasti Medik.

ACALYPHA L. (Euphorbiaceae)

Acalypha rhomboidea Raf. $=$ A. virginica var. rhomboidea (Raf.) Cooperrider

A. virginica L., Three-seeded Mercury var. rhomboidea (Raf.) Cooperrider $=$ A. rhomboidea Raf. var. virginica

ACER L. (Aceraceae)

*A. campestre L., Hedge-maple

Acer dasycarpum Ehrh. = Acer saccharinum L.

Acer eriocarpum Michx. $=$ Acer saccharinum L.

*A. ginnala Maxim., Amur Maple = A. tataricum L. var. ginnala Maxim.

Acer glaucum Marsh. = Acer saccarinum L.

*A. negundo L., Box Elder, Ash-leaf Maple, Manitoba Maple

$=$ A. negundo var. violaceum Mill.

= Negundo negundo (L.) Karst.

A. nigrum Michx.f., Black Maple

= A. saccharum var. nigrum (Michx.f.) Britt.

A. pensylvanicum L., Striped Maple, Moosewood, Green-striped Maple, Whistlewood

*A. platanoides L., Norway Maple

*A. pseudoplatanus L., Sycamore-maple
A. rubrum L., Red Maple, Swamp Maple, Scarlet Maple

var. rubrum

var. trilobum Torr. \& Gray ex Koch, Threelobed red maple.

$=$ A. rubrum var. tridens Wood

A. rubrum $\times$ saccharinum $=$ A. $\times$ freemanii Murr., Soft Maple, Hybrid Swamp Maple

A. saccharinum L., Silver Maple, White Maple, River Maple

$=$ A. dasycarpum Ehrh.

= A. eriocarpum Michx.

= A. glaucum Marsh.

Acer saccharinum Wang. = Acer saccharum Marsh.

A. saccharum Marsh., Sugar Maple, Rock Maple, Hard Maple

= A. saccharum var. schneckii Rehd.

$=$ A. saccharinum Wang.

A. spicatum Lam., Mountain Maple

Acer tataricum L. var. ginnala Maxim. = Acer ginnala Maxim.

Acerates viridiflora (Raf.) Eat. $=$ Asclepias viridiflora $\mathrm{L}$.

ACHILLEA L. (Asteraceae)

*A. millefolium L., Common Yarrow, Milfoil

*var. millefolium

*var. occidentalis DC., Woolly Milfoil

$=$ A. asplenifolia of authors, not Vent.

$=$ A. millefolium ssp. lanulosa (Nutt.) Piper

= A. lanulosa Nutt.

= A. occidentalis (DC.) Ref. ex Rydb.

$=$ A. tomentosa Pursh

*A. ptarmica L., White Tansy Sneezeweed

Achillea tomentosa Pursh $=$ Achillea millefolium var. occidentalis DC.

ACINOS Mill. (Lamiaceae)

*A. arvensis (Lam.) Dandy, Mother of Thyme $=$ Clinopodium acinos (L.) Kuntze

$=$ Satureja acinos (L.) Scheele

ACORUS L. (Araceae)

A. americanus (Raf.) Raf., Calamus, Sweet Flag, Flagroot 
= A. calamus of American authors, not L.

Acrostichum thelypteris L. $=$ Thelypteris palustris Schott

ACTAEA L. (Ranunculaceae)

A. pachypoda Ell., White Baneberry, Doll's Eyes, White Cohosh

= A. alba of American authors, not (L.) Mill.

$=$ A. spicata var. alba L.

A. pachypoda $\times$ spicata $\mathrm{ssp}$. rubra, Baneberry

$=$ A. $\times$ ludovicii Boivin

$=\mathrm{A}$. alba $\times$ rubra

$=\mathrm{A}$. pachypoda $\times$ rubra

A. spicata L., Red Baneberry, Baneberry, Black Cohosh, Snakeberry

ssp. rubra (Ait.) Hultén

$=$ A. rubra (Ait.) Willd.

= A. spicata var. rubra Ait.

Actaea spicata var. alba L. = Actaea pachypoda Ell.

Actinomeris alternifolia (L.) DC. = Verbesina alternifolia (L.) Britt.

ADIANTUM L. (Pteridaceae)

A. pedatum L., Maidenhair Fern

Adicia fontana Lunell = Pilea fontana (Lunnell) Rydb.

Adicia pumila (L.) Raf. = Pilea pumila (L.) A. Gray

ADLUMIA Raf. (Fumariaceae)

A. fungosa (Ait.) Greene ex BSP., Allegheny

Vine, Mountain Fringe, Climbing

Fumitory

$=$ A. asiatica Ohwi

= A. cirrhosa Raf.

$=$ Fumaria fungosa Ait.

$=$ F. recta Raf.

AEGILOPS L. (Poaceae)

*A. cylindrica Host, Jointed Goatgrass

AEGOPODIUM L. (Apiaceae)

*A. podagraria L., Goutweed, Goat's-foot

AESCULUS L. (Hippocastanaceae)

*A. hippocastanum L., Horse-chestnut

*A. glabra Willd., Ohio Buckeye
AETHUSA L. (Apiaceae)

*A. cynapium L., Fool's Parsley

AGALINIS Raf. (Scrophulariaceae)

A. paupercula (Gray) Britt., Small-flowered Purple Gerardia, False-foxglove, Gerardia var. borealis Pennell

$=$ Gerardia paupercula var. borealis (Pennell) Deam $=\mathrm{G}$. purpurea var. parviflora Benth.

A. tenuifolia (Vahl) Raf., Slender Gerardia var. parviflora (Nutt.) Pennell

$=$ Gerardia tenuifolia var. parviflora Nutt. var. tenuifolia

$=$ Gerardia tenuifolia Vahl

AGASTACHE Clayt. ex Gronov. (Lamiaceae)

A. nepetoides (L.) Kuntze, Yellow Giant Hyssop $=$ Lophanthus nepetoides (L.) Benth.

A. scrophulariifolia (Willd.) Kuntze, Purple Giant Hyssop

$=$ A. scrophulariifolia var. mollis (Fern.) Heller

= A. urticifolia sensu House (1924), not (Benth.) Kuntze

$=$ Lophanthus scrophulariaefolius (Willd.) Benth.

Ageratina altissima (L.) R. King \& H. Robinson = Eupatorium rugosum Houtt.

AGRIMONIA L. (Rosaceae)

Agrimonia bicknellii of NY reports, not (Kearney)

Rydb. ex Bickn. $=$ Agrimonia pubescens

Wallr.

*A. eupatoria L., Common Agrimony, Cocklebur Agrimonia eupatoria of Ontario reports, not L. = Agrimonia striata Michx. (q.v.)

A. gyrosepala Wallr., Tall Hairy Agrimony, Agrimony, Cocklebur, Harvest Lice, Stickseed

A. parviflora Solander ex Ait., Small-flowered Agrimony, Agrimony, Cocklebur

A. pubescens Wallr., Soft Agrimony

$=$ A.bicknellii of NY reports, not (Kearney) Rydb. ex Bickn.

$=$ A. mollis (Torr. \& A. Gray) Britt.

A. rostellata Wallr., Agrimony

A. striata Michx., Grooved Agrimony, Britton's Agrimony, Harvest Lice, Cocklebur 
= A. eupatoria of Ontario reports, not L.

AGROPYRON Gaertn. (Poaceae)

Agropyron caninum (L.) Beauv. = Elymus

trachycaulus (Link) Gould ex Shinners ssp.

subsecundus (Link) Barkworth \& D.

Dewey

Agropyron caninum var. tenerum (Vasey) Pease \&

Moore $=$ Elymus trachycaulus (Link)

Gould ex Shinners ssp. trachycaulus

*A. cristatum L., Crested Wheatgrass

$=$ A. desertorum (Fisch. ex Link) Schultes

Agropyron desertorum (Fisch. ex Link) Schultes =

Agropyron cristatum L.

Agropyron repens (L.) Beauv. = Elytrigia repens

(L.) Nevski

Agropyron repens (L.) Beauv. var. littoreum

Anderss. = Elytrigia repens (L.) Nevski

Agropyron repens (L.) Beauv. var. nemorale

Anderss. $=$ Elytrigia repens $($ L. $)$ Nevski

Agropyron repens (L.) Beauv. var. pilosum Scribn.

$=$ Elytrigia repens $($ L.) Nevski

Agropyron smithii Rydb. = Pascopyrum smithii

(Rydb.) Á. Löve

Agropyron tenerum Vasey $=$ Elymus trachycaulus

(Link) Gould ex Shinners ssp. trachycaulus

Agropyron trachycaulum (Link) Malte ex Lewis =

Elymus trachycaulus (Link) Gould ex

Shinners ssp. trachycaulus

Agropyron trachycaulum var. glaucum (Pease \&

Moore) Malte $=$ Elymus trachycaulus

(Link) Gould ex Shinners ssp. subsecundus

(Link) Barkworth \& D. Dewey

Agropyron trachycaulum var. majus (Vasey) Fern.

= Elymus trachycaulus (Link) Gould ex

Shinners ssp. subsecundus (Link)

Barkworth \& D. Dewey

Agropyron trachycaulum var. novae-angliae

(Scribn. ex Brainerd) Fern. = Elymus

trachycaulus (Link) Gould ex Shinners ssp.

trachycaulus

Agropyron trachycaulum var. unilaterale (Vasey)

Malte $=$ Elymus trachycaulus (Link) Gould ex Shinners ssp. trachycaulus

Agrypyron violaceum (Hornem.) Lange $=$ Elymus trachycaulus (Link) Gould ex Shinners ssp. trachycaulus

AGROSTEMMA L. (Caryophyllaceae)
Agrostemma coronaria L. = Lychnis coronaria (L.) Desr.

*A. githago L., Corn-Cockle, Corn-rose, Purple Cockle

= Lychnis githago (L.) Scop.

AGROSTIS L. (Poaceae)

Agrostis alba of authors, not L. = Agrostis gigantea Roth

Agrostis alba var. maritima (Lam.) G.F.W. Mey. = Agrostis stolonifera L. var. palustris

(Huds.) Farw.

Agrostis alba var. palustris (Huds.) Pers. = Agrostis stolonifera L. var. palustris (Huds.) Farw.

*A. capillaris L., Rhode Island Bent, Colonial Bent

$=$ A. tenuis Sibth.

$=\mathrm{A}$. vulgaris With.

Agrostis geminata Trin.$=$ Agrostis scabra Willd .

*A. gigantea Roth, Redtop, Black Bent

= A. gigantea var. dispar (Michx.) Philipson

= A. stolonifera var. major (Gaud.) Farw.

$=\mathrm{A}$. alba of authors, not L.

A. hyemalis (Walt.) BSP., Hair-grass, Southern Hairgrass, Ticklegrass

Agrostis maritima Lam. = Agrostis stolonifera var. palustris (Huds.) Farw.

Agrostis palustris Huds. $=$ Agrostis stolonifera L . var. palustris (Huds.) Farw.

A. perennans (Walt.) Tuckerm., Upland Bent, Autumn

$=$ A. schweinitzii Trin.

A. scabra Willd., Hairgrass, Fly-away Grass, Ticklegrass

$=$ A. geminata Trin.

$=\mathrm{A}$. hyemalis var. tenuis (Tuckerman) Gleason

$=$ A. peckii House

$=$ A. scabra var. geminata (Trin.) Swallen

Agrostis schweinitzii Trin. $=$ Agrostis perennans (Walt.) Tuckerm.

*A. stolonifera L., Creeping Bent, Carpet Bent var. palustris (Huds.) Farw.

= A. alba var. maritima (Lam.) G.F.W. Mey.

$=$ A. alba var. palustris (Huds.) Pers.

= A. maritima Lam.

$=\mathrm{A}$. palustris Huds.

$=\mathrm{A}$. palustris var. stricta 
= A. stolonifera var. compacta Hartm.

var. stolonifera

Agrostis tenuis Sibth. $=$ Agrostis capillaris L.

AILANTHUS Desf. (Simaroubaceae):

*A. altissima (Mill.) Swingle, Tree of Heaven, Ailanthus, Copal Tree

$=$ A. glandulosa Desf.

Aira cespitosa L. = Deschampsia cespitosa (L.)

Beauv.

Aira flexuosa L. = Deschampsia flexuosa (L.) Trin.

ALCEA L. (Malvaceae)

*A. rosea L., Garden Hollyhock

$=$ Althaea rosea $(\mathrm{L}$.$) Cav.$

ALCHEMILLA L. (Rosaceae)

*A. monticola Opiz, Lady's Mantle

$=\mathrm{A}$. vulgaris of $\mathrm{NY}$ reports, not $\mathrm{L}$.

ALISMA L. (Alismataceae)

Alisma geyeri Torr. = Alisma gramineum Lej.

A. gramineum Lej., Water-plantain, Grassyleaved Water-plantain

$=\mathrm{A}$. geyeri Torr.

A. subcordatum Raf., Water-plantain

$=$ A. plantago-aquatica of American authors in part, not L.; sensu Zenkert, 1934 p. 88.

$=$ A. plantago-aquatica var. parviflorum

(Pursh) Torrey (of Eaton \& Schrot 1987 p.124).

$=$ A. plantago-aquatica ssp. subcordatum (Raf.) Hultén

ALLIARIA Heist. ex Fabr. (Brassicaceae)

*A. petiolata (Bieb.) Cav. \& Grande, Garlic

Mustard, Hedge-garlic

$=$ A. alliaria (L.) Britt.

$=$ A. officinalis Andrz. ex Bieb.

$=$ Erysimum alliaria L.

Allionia nyctaginea Michx. = Mirabilis nyctaginea (Michx.) MacM.

ALLIUM L. (Liliaceae)

A. burdickii (Hanes) A. Jones, Wild Leek

$=\mathrm{A}$. tricoccum var. burdickii Hanes

A. canadense L., Wild Garlic, Wild Onion

*A. cepa L., Garden Onion

A. cernuum Roth, Wild Onion
A. tricoccum Ait., Wild Leek, Ramp

Allium tricoccum var. burdickii $=$ Allium burdickii (Hanes) A. Jones

*A. vineale L., Field Garlic, Scallion

Allosorus atropurpureus (L.) Kunze ex Presl. = Pellaea atropurpurea (L.) Link

ALNUS Mill. (Betulaceae)

*A. glutinosa (L.) Gaertn., Black Alder, European Alder

$=$ A. alnus (L.) Britt.

$=\mathrm{A}$. vulgaris Hill

A. incana (L.) Moench, Speckled Alder, Hoary Alder

ssp. rugosa (DuRoi) Clausen, Hazel Alder

$=$ A. rugosa (DuRoi) Spreng.

$=$ A. serrulata (of Day, 1882)

ALOPECURUS L. (Poaceae)

A. aequalis Sobol, Short-awned Foxtail

$=\mathrm{A}$. aristulatus Michx.

$=$ A. geniculatus (of Day, 1882)

*A. geniculatus L., Marsh-foxtail, Water-foxtail

$=$ A. pallescens Piper

Alopecurus pallescens Piper $=$ Alopecurus geniculatus L.

*Alopecurus pratensis L., Meadow Foxtail

Alsine aquatica (L.) Britt. = Myosoton aquaticum (L.) Moench

Alsine marginata (DC. ex Lam. \& DC.) Reichenb. = Spergularia media (L.) C. Presl ex Griseb.

Alsine media (L.) Druce $=$ Spergularia media (L.) C. Presl ex Griseb.

Alsine rubra (L.) Crantz = Spergularia rubra (L.) J. \& C. Presl

ALTHAEA L. (Malvaceae)

*A. officinalis L., Marsh Mallow, White Mallow

Althaea rosea (L.) Cav. = Alcea rosea L.

ALYSSUM L. (Brassicaceae)

*A. alyssoides (L.) L., Yellow Alyssum $=$ A. calycinum $\mathrm{L}$.

= Clypeola allyssoides L.

Alyssum incanum L. = Berteroa incana (L.) DC.

AMARANTHUS L. (Amaranthaceae)

*A. albus L., Tumbleweed, Pale Amaranth 
= A. graecizans of some authors, not L.

A. blitoides S. Wats., Tumbleweed, Prostrate Amaranth

$=\mathrm{A}$. graecizans of some authors, not $\mathrm{L}$.

*A. blitum L., Purplish Amaranth, Livid Amaranth

$=\mathrm{A}$. ascendens Loisel

$=\mathrm{A}$. lividus $\mathrm{L}$.

Amaranthus chlorostachys Willd. $=$ Amaranthus hybridus L.

[*Amaranthus cruentus L., Blood Amaranth, Purple Amaranth, Zander and Pierce (1979) report this species as a synonym of A. hybridus L. Clemants (1992) recognized it as a species derived "probably through selective cultivation of A. hybridus." He does not, however, offer a record of its occurrence in western New York: excluded.]

Amaranthus graecizans of American authors in part, not L. = Amaranthus albus L.

Amaranthus graecizans of American authors in part, not L. = Amaranthus blitoides S. Wats.

*A. hybridus L., Green Amaranth, Pigweed, Wild Beet, Prince's-Feather

$=$ A. chlorostachys Willd.

= A. incurvatus Ti ex Gren. \& Godr.

$=$ A. patulus Berol.

*A. hypochondriacus L., Prince's Feather

Amaranthus incurvatus Ti ex Gren. \& Godr. = Amaranthus hybridus L.

Amaranthus lividus L. = Amaranthus blitum L.

Amaranthus patulus Berol. $=$ Amaranthus hybridus L.

*A. powellii S. Wats., Amaranth = A. retroflexus L. var. powellii (S. Wats.) Boivin

*A. retroflexus L., Green Amaranth, Pigweed, Wild Beet, Redroot Amaranth

Amaranthus retroflexus L. var. powellii (S. Wats.) Boivin = Amaranthus powellii S. Wats.

*A. rudis Sauer, Water-hemp = Acnida tamariscinus Nutt. $=$ Amaranthus tamariscinus (Nutt.) Wood

*A. spinosus L., Spiny Amaranth, Thorny Amaranth

*A. tuberculatus (Moq. ex DC.) Sauer, Roughfruited Water-hemp, Tall Water-hemp
$=$ A. altissimus Riddell

$=$ Montelia tamariscina (Nutt.) A. Gray

AMBROSIA L. (Asteraceae)

A. artemisiifolia L., Common Ragweed, Ragweed, Hogweed, Bitterweed

$=\mathrm{A}$. artemisiifolia varieties

= A. elatior L. var. artemisiifolia (L.) House

*A. coronopifolia Torr. \& A. Gray, Western

Ragweed

$=$ Ambrosia psilostachya DC.

= Ambrosia psilostachya DC. var. coronopifolia (Torr. \& A. Gray) Farw.

Ambrosia elatior L. var. artemisiifolia (L.) House = Ambrosia artemisiifolia L.

Ambrosia psilostachya DC. $=$ Ambrosia coronopifolia Torr. \& A. Gray

A. trifida L., Great Ragweed, Giant Ragweed, Great Ragweed, Buffalo-weed

$=$ A. trifida var. integrifolia (Muhl.) Torr. $\&$

A. Gray

AMELANCHIER Medik. (Rosaceae)

Amelanchier amabilis Wieg. $=$ Amelanchier sanguinea (Pursh) DC.

A. arborea (Michx.f.) Fern., Tree Shadbush, Serviceberry

Amelanchier arborea var. laevis (Wieg.) Ahles = Amelanchier laevis Wieg.

A. canadensis (L.) Medik., Canadian Shadbush, Serviceberry, Shadbush, Juneberry

= A. canadensis var. botryapium Torr. \& A . Gray, sec. House 1924.

= A. canadensis var. oblongifolia Torr. \& A. Gray

= A. oblongifolia (Torr. \& A. Gray) Roemer Amelanchier canadensis var. rotundifolia Torr. \& A. Gray, sec. House 1924 = Amelanchier sanguinea (Pursh.) DC.

A. humilis Wieg., Bush Juneberry

A. intermedia Spach, Serviceberry $=$ A. arborea $\times$ canadensis

A. laevis Wieg., Smooth Shadbush = A. arborea var. laevis (Wieg.) Ahles

Amelanchier oblongifolia (Torr. \& A. Gray) Roemer $=$ Amelanchier canadensis $(\mathrm{L}$. Medik.

A. sanguinea (Pursh.) DC., Round-leaved Juneberry 
= A. amabilis Wieg.

= A. canadensis var. rotundifolia Torr. \& A. Gray, sec. House 1924.

$=$ A. sanguinea var. grandiflora (Wieg.) Rehd.

Amelanchier sanguinea var. grandiflora (Wieg.) Rehd. $=$ Amelanchier sanguinea (Pursh) DC.

A. stolonifera Wieg., Serviceberry

$=$ A. spicata (Lam.) Koch in part

AMMOPHILA Host (Poaceae)

Ammophila arenaria (of authors) $=$ Ammophila breviligulata Fern.

A. breviligulata Fern., Beach Grass, Dunegrass $=$ A. arenaria (of authors)

[Note: Calamagrostis arenaria Roth. of Gray's Manual ed. 5 (1862) has Arundo L. and Psamma Beauv. as generic synonyms (p. 616). House (1924) gives Psamma as an earlier synonym of Ammophila, hence Ammophila arenaria Am. Auth., not A. arenaria (L.) Link, of Europe = Ammophila breviligulata Fernald, according to House.]

AMORPHA L. (Fabaceae)

*A. fruticosa L., False Indigo, Indigo-bush (Regl. M. Niag. Oldham 1999).

Ampelamus albidus (Nutt.) Britt. = Cynanchum laeve (Michx.) Pers.

AMPELOPSIS Michx. (Vitaceae)

*A. brevipedunculata (Maxim.) Trautv., Porcelain Berry

Ampelopsis quinquefolia (L.) Michx. = Parthenocissus quinquefolia (L.) Planch ex DC.

Amphicarpa of authors $=$ Amphicarpaea

AMPHICARPAEA Ell. ex Nutt. (Fabaceae) Amphicarpaea bracteata var. comosa (L.) Fern. = Amphicarpaea bracteata (L.) Rickett \& Stafleu

Amphicarpaea comosa (L.) G. Don = Amphicarpaea bracteata (L.) Rickett \& Stafleu
Amphicarpaea monoica (L.) Ell. = Amphicarpaea bracteata (L.) Rickett \& Stafleu

A. bracteata (L.) Rickett \& Stafleu, Hog Peanut $=$ A. monoica (L.) Ell.

$=$ A. bracteata var. comosa (L.) Fern.

= A. comosa (L.) G. Don

$=$ Falcata comosa (L.) Kuntze

$=$ Glycine bracteata $\mathrm{L}$.

$=\mathrm{G}$. comosa $\mathrm{L}$.

Amygdalus persica L. $=$ Prunus persicaria (L.) Batsch

Anacharis canadensis (Rich. ex Michx.) Planch. = Elodea canadensis Rich. ex Michx.

Anacharis planchonii (Casp.) Rydb. = Elodea canadensis Rich. ex Michx.

ANAGALLIS L. (Primulaceae)

*A. arvensis L., Scarlet Pimpernel, Poor-man'sWeatherglass, Shepherd's-clock

ANAPHALIS DC. (Asteraceae)

A. margaritacea (L.) Benth. \& Hook.f. ex Clarke, Pearly Everlasting

$=$ Antennaria margaritacea (L.) R. Br.

$=$ A. margaritacea subspecies and varieties

Anchistea virginica (L.) Presl $=$ Woodwardia virginica (L.) Sm.

ANCHUSA L. (Boraginaceae)

*A. arvensis (L.) Bieb., Small Bugloss = Lycopsis arvensis L. of Zenkert (1934), Zander \& Pierce (1979), Gleason (1952)

Anchusa myosotidiflora Lehm. = Brunnera macrophylla (Adams) I. M. Johnston

ANDROMEDA L. (Ericaceae)

Andromeda baccata Wang = Gaylussacia baccata (Wang.) Koch

Andromeda calyculata L. = Chamaedaphne calyculata (L.) Moench

Andromeda canescens Small $=$ Andromeda glaucophylla Link.

A. glaucophylla Link., Bog Rosemary, Moorwort $=$ A. canescens Small

= A. polifolia L. ssp. glaucophylla (Link) Hultén

= A. polifolia L. var. angustifolia Ait.

= A. polifolia L. var. glaucophylla (Link.) DC. 
= A. polifolia L. var. latifolia Ait.

= A. rosmarinifolia Pursh, not Gilib.

Andromeda rosmarinifolia Pursh, not Gilib. = Andromeda glaucophylla Link.

ANDROPOGON L. (Poaceae)

Andropogon furcatus Muhl. $=$ Andropogon gerardii Vitm.

A. gerardii Vitm., Big Bluestem, Turkey-foot $=$ A. furcatus Muhl. of Zenkert 1934

Andropogon scoparius Michx. var. scoparius = Schizachyrium scoparium (Michx.) Nash ssp. scoparium

Andropogon scoparius var. frequens Hubbard $=$ Schizachyrium scoparium (Michx.) Nash ssp. scoparium

Andropogon scoparius var. glaucescens House = Schizachyrium scoparium (Michx.) Nash ssp. scoparium

Andropogon scoparius var. septentrionalis Fern. \& Grisc. $=$ Schizachyrium scoparium

(Michx.) Nash ssp. scoparium

A. virginicus L., Broomsedge

ANEMONE L. (Ranunculaceae)

Anemone aconitifolia Michx. $=$ Anemone canadensis $\mathrm{L}$.

Anemone acutiloba (DC.) Lawson = Hepatica nobilis Mill. var. acuta (Pursh) Steyerm.

A. canadensis L., Canada Anemone, Wind-Flower $=$ A. aconitifolia Michx.

$=$ A. pennsylvanica $\mathrm{L}$.

$=$ A. dichotoma (of authors)

A. cylindrica Gray, Long-fruited Anemone, Thimbleweed, Thimble-head Anemone

Anemone dichotoma (of authors) $=$ Anemone canadensis L.

Anemone groenlandica Oed. $=$ Coptis trifolia $(\mathrm{L}$. Salisb.

Anemone hepatica (of authors) $=$ Hepatica nobilis Mill. var. obtusa (Pursh) Steyerm.

Anemone nemorosa var. quinquefolia (L.) Pursh = Anemone quinquefolia L.

Anemone pennsylvanica L. = Anemone canadensis L.

A. quinquefolia L., Wood Anemone, Snowdrops $=$ A. nemorosa var. quinquefolia (L.) Pursh

Anemone thalictroides L. $=$ Thalictrum thalictroides (L.) Eames \& Boivin
A. virginiana L., Thimbleweed, Tall Anemone var. alba (Oakes) Wood

$=$ A. riparia Fern.

= A. virginiana var. riparia (Fern.) Boivin var. virginiana

Anemonella thalictroides (L.) Spach = Thalictrum thalictroides (L.) Eames \& Boivin

Anethum in part $=$ Foeniculum according to A. Wood (no date) The American Botanist and Florist, Part IV

ANETHUM L. (Apiaceae)

Anethum foeniculum of Clinton's Journal, 1862, Oct. 9 = Foeniculum vulgare Mill.

*A. graveolens L., Dill

ANGELICA L. (Apiaceae)

A. atropurpurea L., Purple-stemmed Angelica, Alexanders

$=$ A. atropurpurea var. occidentalis Fassett

= Archangelica. atropurpurea Hoffm.

A. venenosa (Greenway) Fern., Hairy Angelica, Deadly Angelica

$=$ A. villosa (Walt.) BSP

$=$ Archangelica hirsuta Torr. \& A. Gray

Annona triloba L. = Asimina triloba (L.) Dunal

ANTENNARIA Gaertn. (Asteraceae)

Antennaria arnoglossa Greene $=$ Antennaria plantaginifolia (L.) Richards.

Antennaria brainerdii Fern. = Antennaria plantaginifolia (L.) Richards.

Antennaria canadensis Greene $=$ Antennaria neglecta Greene

Antennaria fallax Greene and var. ambigens $($ Greene $)$ Fern. $=$ Antennaria plantaginifolia (L.) Richards.

Antennaria howellii Greene, including ssp. canadensis (Greene) Bayer, ssp. petaloidea (Fern.) Bayer and ssp. neodioica (Greene) Bayer $=$ Antennaria neglecta Greene

Antennaria margaritacea (L.) R. Br. = Anaphalis margaritacea (L.) Benth.\& Hook.f. ex Clarke

Antennaria munda Fern. $=$ Antennaria plantaginifolia (L.) Richards.

A. neglecta Greene, Everlasting, Pussy's-toes

$=\mathrm{A}$. canadensis Greene

$=$ A. grandis (Fern.) House 
$=\mathrm{A}$. howellii Greene

$=$ A. howellii Greene ssp. canadensis (Greene) Bayer

$=$ A. howellii Greene ssp. neodioica (Greene) Bayer

$=$ A. howellii Greene ssp. petaloidea (Fern.) Bayer

= A. neglecta ssp. neodioica (Greene) Bayer

= A. neglecta var. attenuata (Fern.) Cronq.

= A. neglecta var. howellii (Greene) Cronq.

= A. neglecta var. randii (Fern.) Cronq.

$=$ A. neglecta var. simplex Peck

$=$ A. neodioica Greene

= A. neodioica Greene ssp. petaloidea (Fern.)

Bayer \& Stebbins

$=$ A. neodioica Greene var. chlorophylla Fern.

$=$ A. oneidica Greene

$=$ A. petaloidea Fern.

$=$ A. petaloidea Fern. var. novaboracensis Fern.

$=\mathrm{A}$. randii Fern.

$=$ A. virginica of New York reports, not Stebbins

"Note: "Under the International Rules of 1981, new combinations will have to be made here at the varietal level" (Mitchell 1986).

Antennaria neodioica Greene, including both ssp. petaloidea (Fern.) Bayer \& Stebbins and var. chlorophylla Fern. = Antennaria neglecta Greene

Antennaria occidentalis Greene $=$ Antennaria plantaginifolia (L.) Richards.

Antennaria oneidica Greene $=$ Antennaria neglecta Greene

Antennaria parlinii Fern. and ssp. fallax (Greene)

Bayer \& Stebbins $=$ Antennaria

plantaginifolia (L.) Richards.

Antennaria parlinii var. arnoglossa (Greene) Fern. $=$ Antennaria plantaginifolia (L.) Richards.

Antennaria petaloidea Fern. and var. novaboracensis Fern. $=$ Antenaria neglecta Greene

Antennaria petiolata Fern. $=$ Antennaria plantaginifolia (L.) Richards.

A. plantaginifolia (L.) Richards., Everlasting, Pussy's-toes, Lady's Tobacco

= A. arnoglossa Greene

$=\mathrm{A}$. brainerdii Fern.
= A. fallax Greene

$=$ A. fallax Greene var. ambigens (Greene) Fern.

$=$ A. munda Fern.

$=$ A. occidentalis Greene

= A. parlinii Fern. (ssp. parlinii)

= A. parlinii Fern. ssp. fallax (Greene) Bayer \& Stebbins

$=$ A. petiolata Fern.

= A. plantaginifolia var. ambigens (Greene) Cronq.

$=$ A. plantaginifolia var. arnoglossa (Greene) Cronq.

$=$ A. plantaginifolia var. petiolata (Fern.) Heller

$=$ A. solitaria Rydb.

"Note: Under the International Rules of 1981, new combinations will have to be made here at the varietal level," (Mitchell 1986).

Antennaria randii Fern. $=$ Antennaria neglecta Greene

Antennaria solitaria Rydb. $=$ Antennaria plantaginifolia (L.) Richards.

Antennaria virginica of New York reports, not Stebbins $=$ Antennaria neglecta Greene

ANTHEMIS L. (Asteraceae)

*A. arvensis L., Corn Chamomile, Dog Fennel $=\mathrm{A}$. arvensis var. agrestis (Wallr.) DC. $=$ Chamemelum arvense (L.) Schreb.

*A. cotula L., Mayweed, Stinking Chamomile, Stinkweed, Dogweed, Dog Fennel = Maruta cotula (L.) DC.

*A. tinctoria L., Yellow Chamomile, Dyer's Chamomile

$=$ Chamemelum tinctorium (L.) Schreb .

$=$ Cota tinctoria $($ L.) Gay

ANTHOXANTHUM L. (Poaceae)

*A. odoratum L., Sweet Vernal Grass

ANTHRISCUS Pers. emend. Hoffm. (Apiaceae)

*A. cerefolium (L.) Hoffm., Chervil

*A. sylvestris (L.) Hoffm., Wild Chervil

= Chaerophyllum sylvestre L.

Anychia divaricata Raf. $=$ Paronychia fastigiata (Raf.) Fern.

Anychia polygonoides Raf. $=$ Paronychia fastigiata (Raf.) Fern. 
Apalanthe schweinitzii Planch. = Elodea canadensis Rich. ex Michx.

Apargia hispida (L.) Willd. = Leontodon hispidus L.

Apargia nudicaulis (of authors) $=$ Leontodon taraxacoides (Vill.) Merat

Aphyllon uniflorum (L.) Torrey \& Asa Gray ex Asa Gray $=$ Orobanche uniflora L.

APIOS Fabr. (Fabaceae)

A. americana Medik., Groundnut, Wild Bean, Potato-bean, Creek-potato

$=$ A. tuberosa Moench

$=$ Glycine apios L.

Apios tuberosa Moench $=$ Apios americana Medik .

APLECTRUM (Nutt.) Torrey (Orchidaceae)

A. hyemale (Muhl. ex Willd.) Nutt., Putty-root Orchid, Adam-and-Eve

APOCYNUM L. (Apocynaceae)

A. androsaemifolium L., Spreading Dogbane, Pink Dogbane, Indian Hemp

$=\mathrm{A}$. androsaemifolium var. incanum A. DC.

$=$ A. scopulorum Greene ex Rydb.

A. androsaemifolium $\times$ cannabinum $=$ A. $\times$ floribundum Greene, Intermediate Dogbane, Indian Hemp

= A. medium Greene of Zenkert 1934

$=\mathrm{A} . \times$ medium Greene

$=$ A. milleri Britt.

A. cannabinum L., Clasping-leaved Dogbane, Indian Hemp, Dogbane var. cannabinum

= A. cannabinum var. glaberrimum A. DC.

= A. cannabinum var. pubescens (Mitchell ex R. Br.) A. DC.

$=\mathrm{A}$. pubescens Mitchell ex R. Br.

var. hypericifolium Gray

$=$ A. sibiricum Jacq.

Apocynum medium Greene of Zenkert $1934=$

[Apocynum androsaemifolium $\times$ cannabinum $=\mathrm{A} . \times$ floribundum Greene $]$

Apocynum pubescens Mitchell ex R. Br. = Apocynum cannabinum L.

Apocynum scopulorum Greene ex Rydb. = Apocynum androsaemifolium L.

Apocynum sibiricum Jacq. = Apocynum cannabinum L. var. hypericifolium Gray
AQUILEGIA L. (Ranunculaceae)

A. canadensis L., Wild Columbine, Red Columbine, Rock-Bells, Meetinghouses 3

$=$ A. canadensis var. coccinea (Small) Munz

$=\mathrm{A}$. coccinea Small

Aquilegia coccinea Small = Aquilegia canadensis L.

*A. vulgaris L., Garden Columbine, Blue or Purple Columbine

ARABIDOPSIS Heynh. (Brassicaceae)

Arabidopsis lyrata (L.) O'Kane \& Al-Shehbaz ssp. lyrata $=$ Arabis lyrata L.

*A. thaliana (L.) Heynh., Mouse-ear Cress

$=$ Arabis thaliana $\mathrm{L}$.

$=$ Arabidopsis thaliana (L.) Britt. ex Britt. \& Brown

$=$ Sisymbrium thalianum (L.) J. Gay \& Monn.

ARABIS L. (Brassicaceae)

Arabis billardieri DC. $=$ Arabis caucasica Schlect. ex Willd.

Arabis brachycarpa (Torrey \& A. Gray) Britt. = [Arabis drummondii $\times$ holboellii $=$ A. $\times$ divaricarpa A. Nels.]

Arabis bulbosa Schreb. ex Muhl. = Cardamine bulbosa (Schreb. ex Muhl.) BSP

A. canadensis L., Sickle-pod

$=$ A. falcata Michx.

= Boerchera Canadensis (L.) Al-Shehbaz

*A. caucasica Schlect. ex Willd., Wall Rock-cress, Arrowleaf

= A. albida Steven ex Bieb.

$=$ A. alpina L. in part

$=\mathrm{A}$. billardieri DC.

Arabis confinis "as to [Ontario] reports in part, not of S. Watson" is either A. divaricarpa, considered here a hybrid [Arabis drummondii $\times$ holboellii $=$ A. $\times$ divaricarpa A. Nels] or A. drummondii A. Gray according to Morton and Venn (1990).

Arabis dentata (Torr.) Torr. \& A. Gray = Arabis shortii (Fern.) Gleason

Arabis divaricarpa A. Nels. $=[$ Arabis drummondii $\times$ holboellii $=$ A. $\times$ divaricarpa A. Nels.]

A. drummondii A. Gray, Drummond's Rock Cress 
= Boechera drummondii (A. Gray) Á. \& D. Löve

$=$ Turritis stricta Graham, not Arabis stricta Huds. according to House, 1924 p.365

A. drummondii $\times$ holboellii $=$ A. $\times$ divaricarpa A. Nels., Purple Rock-cress

= A. brachycarpa (Torrey \& A. Gray) Britt. Arabis falcata Michx. = Arabis canadensis L.

A. glabra (L.) Bernh., Tower Cress, TowerMustard

$=$ A. perfoliata Lam.

$=$ Turritis glabra L.

A. hirsuta (L.) Scop., Hairy Rock Cress

var. pycnocarpa (Hopk.) Rollins

= A. hirsuta ssp. pycnocarpa (Hopk.) Hultén

= A. pycnocarpa Hopk.

A. laevigata (Muhl. ex Willd.) Poir. ex Lam., Smooth Rock Cress

$=$ Boerchera laevigata (Muhl. ex Willd.) AlShehbaz

A. lyrata L., Lyre-leaved Rock Cress

= Arabidopsis lyrata (L.) O'Kane \& Al-

Shehbaz ssp. lyrata

$=$ Arabis lyrata var. kamschatica Fisch. ex DC.

$=$ Cardamine spathulata Michx.

$=$ Cardaminopsis lyrata (L.) Hiitonen.

Arabis perfoliata Lam. $=$ Arabis glabra (L.) Bernh.

Arabis perstellata var. shortii Fern. $=$ Arabis shortii (Fern.) Gleason

Arabis pycnocarpa Hopk. $=$ Arabis hirsuta (L.)

Scop. var. pycnocarpa (Hopk.) Rollins

Arabis rhomboidea Pers. $=$ Cardamine bulbosa

(Schreb. ex Muhl.) BSP

A. shortii (Fern.) Gleason, Short's Rock Cress,

Toothed Rock-Cress

= A. dentata (Torr.) Torr. \& A. Gray

$=$ A. perstellata var. shortii Fern.

Arabis stricta Huds., see Arabis drummondii A. Gray

Arabis thaliana L. $=$ Arabidopsis thaliana (L.)

Heynh.

ARALIA L. (Araliaceae)

A. hispida Vent., Bristly Sarsaparilla, Dwarf-Elder

A. nudicaulis L., Wild Sarsaparilla

A. racemosa L., Spikenard, Petty-Morrel, Life-ofMan
A. spinosa L., Hercules' Club, Devil's Walking-

Stick, Angelica-Tree, Prickly-Ash

ARCEUTHOBIUM Bieb. (Viscaceae)

A. pusillum C. Peck, Dwarf Mistletoe

$=$ Razoumofskya pusilla (C. Peck) Kuntze

Archangelica atropurpurea Hoffm. = Angelica atropurpurea L.

Archangelica hirsuta Torr. \& A. Gray = Angelica venenosa (Greenway) Fern.

ARCTIUM L. (Asteraceae)

*A. lappa L., Great Burdock = A. major Gaertn.

Arctium lappa var. tomentosa (Mill.) Gray $=$ Arctium tomentosum Mill. excluded (Zander \& Pierce 1979).

Arctium major Gaertn. = Arctium lappa L.

*A. minus (Hill.) Bernh., Common Burdock = A. nemorosum of authors, not Lej. \& Court.

Arctium nemorosum of authors not Lej. \& Court. $=$ Arctium minus (Hill) Bernh.

Arctium tomentosum Mill., excluded (Zander \& Pierce 1979).

ARCTOSTAPHYLOS L. (Ericaceae)

A. uva-ursi (L.) Spreng., Bearberry, Sandberry, Hog-Cranberry, Mealberry, Kinnikinnik, Mountain Box

$=$ A. uva-ursi varieties

ARENARIA L. (Caryophyllaceae)

Arenaria angustifolia Regel $=$ Moehringia lateriflora (L.) Fenzl

Arenaria lateriflora L. = Moehringia lateriflora (L.) Fenzl

Arenaria leptoclados Reichenb. ex Guss. = Arenaria serpyllifolia L.

Arenaria marginata DC. ex Lam. \& DC. = Spergularia media (L.) C. Presl ex Griseb.

Arenaria maritima Pall. $=$ Spergularia media $($ L.)

C. Presl ex Griseb.

Arenaria media L. = Spergularia media (L.) C.

Presl ex Griseb.

Arenaria rubra L. $=$ Spergularia rubra (L.) J. \& C.

Presl

*A. serpyllifolia L., Thyme-leaved Sandwort $=$ A. leptoclados Reichenb. ex Guss. 
= A. serpyllifolia ssp. leptoclados (Reichenb. ex Guss.) Nymer

= A. serpyllifolia var. tenuior Mert. \& Koch

$=$ A. tenuior (Mert. \& Koch) Gürke

Arenaria serpyllifolia ssp. leptoclados (Reichenb. ex Guss.) Nymer and var. tenuior Mert. \& Koch $=$ Arenaria serpyllifolia L .

Arenaria tenuior (Mert. \& Koch) Gürke = Arenaria serpyllifolia L.

ARETHUSA L. (Orchidaceae)

A. bulbosa L., Dragon's Mouth Orchid, Swamp Pink

Argentina anserina (L.) Rydb. = Potentilla anserina L. ssp. anserina

Argentina babcockiana Rydb. = Potentilla anserina L. ssp. anserina

ARISAEMA Mart. (Araceae)

A. dracontium (L.) Schott ex Schott \& Endl., Green Dragon, Dragon-root

$=$ Arum dracontium L.

= Muricauda dracontium (L.) Kunth

A. triphyllum (L.) Schott ex Schott \& Endl., Jackin-the-Pulpit, Indian-Turnip

ssp. stewardsonii (Britt.) Huttleston

Stewardson's Jack-in-the-Pulpit

$=$ A. stewardsonii Britt.

$=$ A. triphyllum var. stewardsonii (Britt.)

Stevens.

ssp. triphyllum

$=$ A. atrorubens (Ait.) Blume

$=$ Arum triphyllum L.

ARISTIDIA L. (Poaceae)

*A. dichotoma L., Poverty Grass; Three-awn

*A. oligantha Michx., Prairie Three-awn

ARMORACIA Gaertn., Meyer \& Scherb.

(Brassicaceae)

Armoracia aquatica (Eaton) Wieg. = Armoracia lacustris (A. Gray) Al-Shehbaz \& V. Bates

Armoracia armoracia (L.) Cockerell $=$ Armoracia rusticana (Lam.) Gaertn., Meyer \& Scherb.

A. lacustris (A. Gray) Al-Shehbaz \& V. Bates, Lake Cress, River-Cress

$=$ A. aquatica (Eaton) Wieg.

= Nasturtium lacustre A. Gray

$=$ Neobeckia aquatica (Eaton) Greene
$=$ Rorippa aquatica (Eaton) E. J. Palmer \& Steyermark

Armoracia lapathifolia Gilib. = Armoracia rusticana (Lam.) Gaertn., Mey. \& Scherb.

*A. rusticana (Lam.) Gaertn., Meyer \& Scherb., Horse-radish

$=$ A. armoracia (L.) Cockerell

= A. lapathifolia Gilib.

$=$ Nasturtium armoracia (L.) Fries

Arnoglossum atriplicifolium (L.) H. Robinson = Cacalia atriplicifolia $\mathrm{L}$.

ARONIA Medik. (Rosaceae)

A. arbutifolia (L.) Pers., Red Chokeberry

$=$ Mespilus arbutifolia L.

$=$ Photinia pyrifolia (Lam.) Robertson $\&$ Phipps

$=$ Pyrus arbutifolia (L.) L.f.

$=\mathrm{P}$. arbutifolia var. erythrocarpa Michx.

Aronia arbutifolia var. atropurpurea (Britt.) Seym. $=[$ Aronia arbutifolia $\times$ melanocarpa $=\mathrm{A}$. $\times$ prunifolia (Marsh.) Rehd.]

Aronia arbutifolia var. nigra Willd. $=$ Aronia melanocarpa (Michx.) Ell.

A. melanocarpa (Michx.) Ell., Black Chokeberry $=$ A. arbutifolia var. melanocarpa (Michx.) Torr.

$=$ A. arbutifolia var. nigra Willd.

$=$ A. nigra (Willd.) Köhne

= Mespilus arbutifolia var. melanocarpa Michx.

$=$ Photinia melanocarpa (Michx.) Robertson \& Phipps

$=$ Pyrus arbutifolia var. melanocarpa Michx.

$=$ P. melanocarpa (Michx.) Willd.

Aronia nigra (Willd.) Köhne $=$ Aronia melanocarpa (Michx.) Ell.

A. arbutifolia $\times$ melanocarpa $=$ A. $\times$ prunifolia (Marsh.) Rehd., Purple Chokeberry

$=$ A. arbutifolia var. atropurpurea (Britt.) Seym.

$=$ A. atropurpurea Britt.

$=$ A. prunifolia (Marsh.) Rehd.

$=$ Mespilus prunifolia Marsh.

$=$ Photina floribunda (Lindl.) Robertson \& Phipps

$=$ Pyrus arbutifolia var. atropurpurea (Britt.)

B. Robinson

$=$ P. floribunda Lindl. 
Aronia atropurpurea Britt. $=[$ Aronia arbutifolia $\times$ melanocarpa $=$ A. $\times$ prunifolia (Marsh.) Rehd.]

Aronia prunifolia $($ Marsh. $)$ Rehder $=[$ Aronia arbutifolia $\times$ melanocarpa $=$ A. $\times$ prunifolia (Marsh.) Rehd.]

ARRHENATHERUM Beauv. (Poaceae)

Arrhenatherum avenaceum Beauv., nom. nud. $=$ Arrhenatherum elatius (L.) Beauv. ex Presl \& Presl

*A. elatius (L.) Beauv. ex Presl \& Presl, Tall Oatgrass

$=$ A. avenaceum Beauv., nom. nud.

$=$ A. elatius var. bulbosum (Willd.) Spenner

ARTEMISIA L. (Asteraceae)

*A. abrotanum L., Southernwood, Old-Man $=$ A. procera Willd.

*A. absinthium L., Wormwood, Absinthe, Absinthium

*A. biennis Willd., Biennial Wormwood, SageWeed

A. campestris L., Tall Wormwood, Wild Sage ssp. caudata (Michx.) Hall \& Clem.

= A. caudata Michx.

= A. canadensis of NY reports, not Michx. (and of Day, 1882)

Artemisia canadensis of NY reports, not Michx. (and of Day, 1882) $=$ Artemisia campestris L. ssp. caudata (Michx.) Hall \& Clem.

Artemisia caudata Michx. $=$ Artemisia campestris ssp. caudata (Michx.) Hall \& Clem.

Artemisia gnaphaloides Nutt. $=$ Artemisia ludoviciana Nutt. ssp. ludoviciana

*A. ludoviciana Nutt., Western Mugwort, White Sage, Western Mugwort, Cudweed

*ssp. ludoviciana

$=$ A. ludoviciana varieties including A. gnaphaloides Nutt.

Artemisia procera Willd. $=$ Artemisia abrotanum L.

*A. vulgaris L., Common Mugwort, Felon-Herb

$=\mathrm{A}$. vulgaris varieties

Arum dracontium L. = Arisaema dracontium (L.)

Schott ex Schott \& Endl.

Arum triphyllum L. = Arisaema triphyllum (L.)

Schott ex Schott \& Endl.

ASARUM L. (Aristolochiaceae)
A. canadense L., Wild ginger

= A. acuminatum (Ashe) Bickn.

= A. canadense var. acuminatum Ashe

= A. canadense var. ambiguum (Bickn.) Farw.

= A. canadense var. reflexum (Bickn.) B. Robinson

$=$ A. reflexum Bickn.

ASCLEPIAS L. (Asclepiadaceae)

Asclepias bicknellii Vail = Asclepias exaltata L .

A. exaltata L., Poke Milkweed

$=\mathrm{A}$. bicknellii Vail

$=$ A. phytolaccoides Pursh

A. incarnata L., Swamp Milkweed

Asclepias intermedia Vail = Asclepias syriaca L .

Asclepias phytolaccoides Pursh $=$ Asclepias exaltata L.

A. quadrifolia Jacq., Four-leaved Milkweed

A. syriaca L., Common Milkweed

= A. cornuti Dec. in DC.

$=\mathrm{A}$. intermedia Vail

A. tuberosa L., Butterfly-weed, Pleurisy-Root, Orange Milkweed, Tuber-Root, Chigger-

Flower, Indian-Paintbrush

var. interior (Woodson) Shinners

= A. tuberosa ssp. interior Woodson

= A. tuberosa var. tuberosa of NY reports

A. verticillata L., Whorled Milkweed

A. viridiflora L., Green Milkweed

$=$ Acerates viridiflora (Raf.) Eat.

ASIMINA Adams (Annonaceae)

A. triloba (L.) Dunal, Pawpaw

$=$ Annona triloba $\mathrm{L}$.

ASPARAGUS L. (Liliaceae)

*A. officinalis L., Asparagus

Asperula odorata L. = Galium odoratum (L.) Scop.

Aspidium acrostichoides Sw. = Polystichum acrostichoides (Michx.) Schott.

Aspidium boottii Tuckerm. $=[$ Dryopteris cristata $\times$ intermedia $=$ D. $\times$ boottii (Tuckerm.) Underw.

Aspidium campyloptera Kunze $=$ Dryopteris campyloptera (Kunze) Clarkson

Aspidium cristatum (L.) Sw. = Dryopteris cristata (L.) A. Gray 
Aspidium cristatum var. clintonianum D.C. Eat. = Dryopteris clintoniana (D. Eat. ex A. Gray) Dowell

Aspidium goldianum Hook. ex Goldie $=$ Dryopteris goldiana (Hook. ex Goldie) A. Gray

Aspidium intermedium Muhl. ex Willd. = Dryopteris intermedia (Muhl. ex Willd.) A. Gray

Aspidium marginale (L.) Sw. = Dryopteris marginalis (L.) A. Gray

Aspidium noveboracense (L.) Sw. = Thelypteris noveboracensis (L.) Nieuwl.

Aspidium spinulosum (O.F. Muell.) Watt $=$ Dryopteris carthusiana (Vill.) Fuchs

Aspidium spinulosum var. intermedium (Muhl.) D.C. Eaton $=$ Dryopteris intermedia (Muhl. ex Willd.) A. Gray

Aspidium thelypteris (L.) Sw. = Thelypteris palustris Schott

ASPLENIUM L. (Aspleniaceae)

Asplenium acrostichoides Sw. = Deparia acrostichoides (Sw.) Kato

Asplenium angustifolium (of authors) = Diplazium pycnocarpon (Sprengel) M. Broun

Asplenium ebeneum Ait. $=$ Asplenium platyneuron (L.) BSP.

Asplenium filix-femina of Gray's Manual, ed. $7=$ Athyrium filix-femina (L.) Roth ex Mertens var. angustum (Willd.) Lawson

A. platyneuron (L.) BSP., Ebony Spleenwort $=$ A. ebeneum Ait.

$=$ A. platyneuron var. incisum (Howe ex Peck) B. Robinson

Asplenium pycnocarpon (Sprengel) Tidestr. $=$ Diplazium pycnocapon (Sprengel) M. Broun

A. rhizophyllum L., Walking Fern $=$ A. rhizophyllum of $\mathrm{L}$. and other authors $=$ Camptosorus rhizophyllus (L.) Link.

A. scolopendrium L., Hart's-tongue Fern var. americanum (Fern.) Kartesz \& Gandhi $=$ Phyllitis scolopendrium (L.) Newm. $=\mathrm{P}$. scolopendrium var. americanum Fern.

Asplenium thelypteroides Michx. = Deparia acrostichoides (Sw.) Kato

A. trichomanes L., Maidenhair Spleenwort $=\mathrm{A}$. trichomanes var. quadrivalens D. Meyer
Asprella hystrix (L.) Humb. = Elymus hystrix L. var. hystrix

Asprella hystrix var. bigelowiana Fern. = Elymus hystrix L. var. bigeloviana (Fern.) Bowden

ASTER L. (Asteraceae)

A. acuminatus Michx., Whorled Aster, Mountain Aster, Wood Aster

= Oclemena acuminata (Michx.) Greene

Aster aestivus Aiton was a synonym of A. longifolius Lam. according to House $(1924)=$ Aster praealtus Poir., q.v.

Aster angustus (Lindl.) Torr. \& A. Gray = Brachyactis ciliata (Ledeb.) Ledeb. ssp. angusta (Lindl.) A. Jones

Aster azureus Lindl. ex Hook. $=$ Aster oolentangiensis Ridd.

A. borealis (Torr. \& A. Gray) Prov., Rush Aster, Bog Aster

$=$ A. junciformis Rydb.

$=$ A. tenuifolius (of Day, 1882), not Mitchell \& Tucker (1997).

$=$ Symphyotrichum boreale $($ Torr. $\&$ A . Gray) Á. \& D. Löve

Aster brachyactis Blake $=$ Brachyactis ciliata (Ledeb.) Ledeb. ssp. angusta (Lindl.) A. Jones

Aster carmesinus Burgess ex Britt. \& Brown $=$ Aster divaricatus L.

Aster castaneus Burgess ex Small $=$ Aster divaricatus $\mathrm{L}$.

Aster claytonii Burgess = Aster divaricatus L .

Aster concinnus Willd. = Aster laevis L var. concinnus (Willd.) House

A. cordifolius L., Heart-leaved Aster, Blue Wood Aster

$=\mathrm{A}$. cordifolius var. polycephalus Porter

$=$ A. cordifolius var. racemiflorus Fern.

= Symphyotrichum cordifolium (L.) Nesom

Aster cordifolius var. incisus Britt. $=$ Aster lowrieanus Porter

Aster cordifolius var. laevigatus Porter $=$ Aster lowrieanus Porter

Aster cordifolius var. lanceolatus Porter $=$ Aster lowrieanus Porter

Aster cordifolius var. sagittifolius (Wedem. ex Willd.) A. Jones $=$ Aster sagittifolius Wedem. ex Willd.

Aster corymbosus Ait. $=$ Aster divaricatus L . 
Aster crenifolius (Fern.) Cronq. = Aster novi-belgii L. var. tardiflorus (L.) A. Jones

A. divaricatus L., White Wood Aster

$=$ A. carmesinus Burgess ex Britt. \& Brown

$=$ A . castaneus Burgess ex Small

$=\mathrm{A}$. claytonii Burgess

$=\mathrm{A}$. corymbosus Ait.

$=\mathrm{A}$. olivaceus Burgess

$=$ A. scutiformis Burgess

$=\mathrm{A}$. tenebrosus Burgess

$=$ Eurybia divaricata (L.) Nesom

A. dumosus L., Bushy Aster, Rice-button Aster $=$ A. dumosus var. coridifolius [sic] (Michx.)

Torr. \& A. Gray

= A. dumosus var. var. strictior Torr. \& A. Gray

Aster eminens Willd. = Aster longifolius Lam.

A. ericoides L., White Heath Aster

$=\mathrm{A}$. ericoides var. prostratus (Kuntze) Blake

$=$ A. multiflorus Ait.

$=$ A. multiflorus Ait. var. exiguus Fern.

$=$ Lasallea ericoides (L.) Semple \& Brouillet

$=$ Symphyotrichum ericoides (L.) Nesom

$=$ Virgulus ericoides (L.) Reveal

Aster ericoides $\times$ novae-angliae $=$ A. $\times$

amethystinus Nutt., Amethyst Wreath

Aster

Aster ericoides var. pringlei A Gray $=$ Aster

pilosus Willd. var. pringlei (Gray) Blake

Aster faxonii Porter $=$ Aster pilosus Willd. var. pringlei (Gray) Blake

Aster hirsuticaulis Lindl. ex DC. $=$ Aster lateriflorus (L.) Britt. var. hirsuticaulis (Lindl. ex DC.) Porter

A. infirmus Michx., Corn-leaved Aster, Cornelleaved Aster

$=$ Doellingeria infirma (Michx.) Greene

Aster interior Wieg. = Aster lanceolatus Willd. var. interior (Wieg.) Semple \& Chmiel.

Aster junceus Ait. = Aster longifolius Lam.

Aster junciformis Rydb. = Aster borealis (Torr. \& A. Gray) Prov.

A. laevis L., Smooth Aster

var. laevis

= A. laevis var. amplifolius Porter

$=$ A. laevis var. cyaneus (Hoffm.) Patterson

= Symphyotrichum leave (L.) Á. \& D. Löve

Aster laevis var. cyaneus (Hoffm.) Patterson $=$ Aster laevis L.
A. lanceolatus Willd., Tall White Aster

var. interior (Wieg.) Semple \& Chmielewski $=\mathrm{A}$. interior Wieg.

$=$ A. lanceolatus $\times$ tradescantii of authors

= A. simplex var. interior (Wieg.) Cronq.

var. lanceolatus

= A. paniculatus Lam.

= Symphiotrichum lanceolatum (Willd.)

Nesom var. lanceolatum

A. lateriflorus (L.) Britt., Starved Aster, Calico

Aster, White Woodland Aster

var. hirsuticaulis (Lindl. ex DC.) Porter

$=$ A. hirsuticaulis Lindl. ex DC.

$=\mathrm{A}$. tradescantii L.

= A. tradescantii L. var. saxatilis (Fern.)

House

$=$ Symphyotrichum tradescantii (L.) Nesom

var. lateriflorus

= A. lateriflorus var. augustifolius Wieg.

= A. lateriflorus var. horizontalis (Desf.)

Farw.

$=$ A. miser Nutt.

= A. vimineus Lam.

$=$ Solidago lateriflora L.

= Symphyotrichum lateriflorum (L.) Á. \& D. Löve

Aster longifolius of some NY reports, not Lam. = Aster praealtus Poir.

A. lowrieanus Porter, Lowrie's Aster

$=$ A. cordifolius var. incisus Britt.

$=\mathrm{A}$. cordifolius var. laevigatus Porter

$=$ A . cordifolius var. lanceolatus Porter

$=\mathrm{A}$. lowrieanus var. incisus (Britt.) Porter

$=\mathrm{A}$. lowrieanus var. lanceolatus Porter

$=$ A. plumarius Burgess ex Britt. \& Brown

$=$ Symphyotrichum lowrieanum (Porter)

Nesom

Aster lucidulus (A. Gray) Wieg. = Aster puniceus var. firmus (Nees) Torr. \& A. Gray

A. macrophyllus L., Large-leaved Aster var. macrophyllus

$=$ A. roscidus Burgess ex Britt. \& Brown

= A. violaris Burgess ex Britt. \& Brown

$=$ Eurybia macrophylla (L.) Cass.

Aster miser Nutt. $=$ Aster lateriflorus (L.) Britt.

Aster multiflorus Ait. $=$ Aster ericoides L .

Aster multiflorus var. exiguus Fern. $=$ Aster ericoides L.

A. nemoralis Ait., Bog Aster 
$=$ Oclemena nemoralis (Ait.) Greene

A. novae-angliae L., New England Aster

$=$ A. novae-angliae f. geneseensis House

$=$ A. novae-angliae var. roseus DC.

$=$ A. roseus Desf. of Gray's Manual, 5th Ed. p. 235

= Lasallea novae-angliae (L.) Semple \&

Brouillet

=Symphyotrichum novae-angliae (L.) Nesom

$=$ Virgulus novae-angliae (L.) Reveal \& Keener

A. novi-belgii L., New York Aster var. tardiflorus (L.) A. Jones, Late-flowering Aster

$=$ A. crenifolius (Fern.) Cronq.

$=$ A. tardiflorus L.

$=$ Symphyotrichum novi-belgii (L.) Nesom var. crenifolium (Fern.) J. Labrecque \& L. Brouillet

Aster olivaceus Burgess = Aster divaricatus L.

A. ontarionis Wieg., Ontario Aster

A. oolentangiensis Ridd., Sky-blue Aster

$=$ A. azureus Lindl. ex Hook

$=\mathrm{A}$. shortii of NY reports, not Lindl.

$=$ Symphyotrichum oolentangiensis (Riddell)

Nesom var. oolentangiensis

Aster paniculatus Lam. $=$ Aster lanceolatus Willd . var. lanceolatus

A. patens Ait., Late Purple Aster

$=$ A. patens var. phlogifolius (Muhl.) Nees

Aster patens var. phlogifolius (Muhl.) Nees = Aster patens Ait.

A. paternus Cronq., White-topped Aster

$=$ Conyza asteroides L.

= Sericocarpus asteroides (L.) BSP.

A. pilosus Willd., Hairy Aster, Heath Aster var. pilosus

$=\mathrm{A}$. ericoides of some authors, not L.

$=$ A. ericoides var. villosus (Michx.) Torr. \& A. Gray

$=$ Symphyotrichum pilosum (Willd.) Nesom var. pilosum

var. pringlei (Gray) Blake

$=$ A. ericoides var. pringlei A. Gray

$=$ A. faxonii Porter

$=$ A. pilosus var. demotus Blake

= A. pringlei (Gray) Britt. ex Britt. \& Brown

Aster plumarius Burgess ex Britt. \& Brown $=$ Aster lowrieanus Porter
A. praealtus Poir., Willow-leaved Aster

$=\mathrm{A}$. aestivus Aiton was a synonym of A. longifolius Lam. according to House (1924)

= A. longifolius of some NY reports, not Lam.

= A. salicifolius Ait., not Lam. (of Zenkert, 1934)

= Symphyotrichum praealtum (Poir.) Nesom var. praealtum

A. prenanthoides Muhl. ex Willd., Crookedstemmed Aster

$=$ Aster prenanthoides var. porrectifolius Porter

$=$ Symphyotrichum prenanthoides (Muhl. ex Willd.) Nesom

Aster pringlei (A. Gray) Britt. ex Britt. \& Brown = Aster pilosus Willd. var. pringlei (A. Gray) Blake

Aster ptarmicoides (Nees) Torr. \& A. Gray = Solidago ptarmicoides (Nees) Boiv.

A. puniceus L., Purple-stemmed Aster

NOTE: Aster vimineus Willd. = Aster puniceus var. vimineus Gray in Gray's Manual of Botany, 5th edition p. 234, thought to be a shade form of A. puniceus.

var. firmus (Nees) Torr. \& A. Gray, Cornel-leaf Aster

$=$ A. firmus Nees.

= A. lucidulus (A. Gray) Wieg.

var. puniceus

$=$ A. puniceus var. compactus Fern.

$=$ A. puniceus var. demissus Lindl.

= Symphyotrichum puniceum (L.) Á. \& D. Löve

Aster roscidus Burgess ex Britt. \& Brown $=$ Aster macrophyllus L. var. macrophyllus

Aster roseus Desf. of Gray's Manual, 5th Ed. p. 235 = Aster novae-angliae L.

A. sagittifolius Wedem. ex Willd., Arrow-leaved Aster

$=\mathrm{A}$. cordifolius var. sagittifolius (Wedem. ex Willd.) A. Jones

$=$ A. hirtellus Lindl., according to NYFA July 2005

= A. urophyllus of NY reports, not Lindl., "fide A. Cronquist, who saw the type specimens," (Mitchell \& Tucker 1997) 
$=$ Symphyotrichum urophyllum (Lindl.) Nesom (the accepted name sec. NYFA July 2005)

Aster salicifolius Ait., not Lam. (of Zenkert, 1934) $=$ Aster praealtus Poir.

Aster salicifolius Willd. not Lam. = Aster longifolius Lam.

A. schreberi Nees, Large-leaf Aster, Schreber's Aster

$=$ A. curvescens Burgess ex Britt. \& Brown

$=$ A. glomeratus (Bernh. ex Nees) Burgess ex Britt. \& Brown

Aster scutiformis Burgess $=$ Aster divaricatus L.

Aster shortii Lindl. $=$ Aster oolentangiensis Ridd .

(Note in Ontario these are separate

(Morton \& Venn, 1990)

Aster simplex Willd. = Aster lanceolatus Willd. var. simplex (Willd.) A. Jones

Aster simplex var. interior (Wieg.) Cronq. $=$ Aster lanceolatus var. interior (Wieg.) Semple \& Chmiel.

Aster tardiflorus L. = Aster novi-belgii L. var. tardiflorus (L.) A. Jones; there is an Aster $\times$ tardiflorus L. $=$ A. cordifolius $\times$ A. puniceus reported for Ontario by Morton and Venn (1990) "reject., reports need confirmation."

Aster tenebrosus Burgess $=$ Aster divaricatus L.

Aster tenuifolius (of Day, 1882) = Aster borealis (Torr. \& A. Gray) Prov.

Aster tradescantii L. $=$ Aster lateriflorus (L.) Britt. var. hirsuticaulis (Lindl. ex DC.) Porter

Aster tradescantii L. var. saxatilis (Fern.) House = Aster lateriflorus (L.) Britt. var. hirsuticaulis (Lindl. ex DC.) Porter

A. umbellatus Mill., Flat-topped White Aster var. umbellatus

$=$ Doellingeria umbellata (Mill.) Nees

$=$ D. umbellata var. flexicaulis House

$=$ D. umbellata var. oneidica House

A. undulatus L., Wavy-leaved Aster

$=\mathrm{A}$. undulatus var. loriformis Burgess ex

Britt. \& Brown

Aster urophyllus Lindl. $=$ Aster sagittifolius Wedem. ex Willd.

Aster vimineus Lam. $=$ Aster lateriflorus (L.) Britt. "Note: The type specimen [of A. vimineus] is apparently a garden hybrid, so this binomial will not stand for our native plants," (Mitchell 1986).

Aster vimineus Lam. is treated as a synonym of A. lateriflorus by Semple and Heard (1987) and Morton and Venn (1990).

Aster vimineus Willd. = Aster puniceus var. vimineus Gray in Gray's Manual of Botany, 5th edition p. 234.

Aster violaris Burgess ex Britt. \& Brown $=$ Aster macrophyllus L. var. macrophyllus

ASTRAGALUS L. (Fabaceae)

A. canadensis L., Canadian Milkvetch $=\mathrm{A}$. carolinianus of authors, not L.

$=$ Phaca canadensis (L.) Kuntze

Astragalus cooperi A. Gray = Astragalus neglectus (Torr. \& A. Gray) Sheldon

*A. glycyphyllos L., European Milk-vetch, Fitsroot

A. neglectus (Torr. \& A. Gray) Sheldon, Cooper's Milk-vetch,

$=$ A. cooperi A. Gray

= Phaca neglecta Torr. \& A. Gray

ATHYRIUM Roth (Dryopteridaceae)

Athyrium acrostichoides (Sw.) Diels = Deparia acrostichoides (Sw.) Kato

Athyrium angustifolium (Michx.) Milde $=$ Diplazium pycnocarpon (Sprengel) M. Broun

Athyrium angustum (Willd.) C. Presl = Athyrium filix-femina var. angustum (Willd.) Lawson

Athyrium asplenioides (Michx.) Desv. of authors in part $=$ Athyrium filix-femina var. angustum (Willd.) Lawson

Athyrium filix-femina (L.) Roth ex Mertens var. angustum (Willd.) Lawson, Northern Lady-fern, Upland Lady-fern

$=$ Aspidium angustum Willd.

= Athyrium angustum (Willd.) C. Presl

$=$ A. asplenioides (Michx.) Desv. of authors in part

$=$ A. filix-femina (L.) Roth var. asplenioides (Michx.) Farw.

$=$ A. filix-femina var. michauxii (Sprengel) Farwell 
Athyrium filix-femina var. michauxii (Sprengel)

Farwell $=$ Athyrium filix-femina var. angustum (Willd.) Lawson

Athyrium filix-femina (L.) Roth var. asplenioides (Michx.) Farw. = Athyrium filix-femina var. angustum (Willd.) Lawson

Athyrium asplenioides (Michx.) Desv. (not Eat.) = Athyrium filix-femina var. asplenioides (Michx.) Farw (excluded)

Athyrium filix-femina (L.) Roth. var. michauxii Mett. $=$ Athyrium filia-femina var. angustum (Willd.) Lawson

Athyrium pycnocarpon $($ Sprengel $)$ Tidestr. = Diplazium pycnocarpon (Sprengel) M. Broun

Athyrium thelypteroides (Michx.) Desv. $=$ Deparia acrostichoides (Sw.) Kato

Atragene americana Sims. $=$ Clematis occidentalis (Hornem.) DC.

ATRIPLEX L. (Chenopodiaceae)

Atriplex angustifolia Sm. = Atriplex patula L.

Atriplex deltoides Babington $=$ Atriplex prostrata

Boucher ex DC. in Lam. \& DC.

Atriplex hastata sensu Aellen, not L. = Atriplex prostrata Boucher ex DC. in Lam. \& DC.

Atriplex littoralis L. = Atriplex patula L.

Atriplex oppositifolia DC. $=$ Atriplex prostrata Boucher ex DC. in Lam. \& DC.

A. patula L., Spreading Orache, Seaside Orach or Atriplex, Spearscale

$=$ A. angustifolia $\mathrm{Sm}$.

$=\mathrm{A}$. littoralis L.

= A. patula var. hastata A. Gray

= A. patula var. littoralis (L.) A. Gray

*A. prostrata Boucher ex DC. in Lam. \& DC., Orach, Spearscale

$=$ A. deltoides Babington

= A. hastata sensu Aellen, not L.

= A. oppositifolia DC.

$=$ A. triangulais Willd.

*A. rosea L., Red Orach, Rosy Orach, Tumbleweed, Tumbling Orach

= A. spatiosa A. Nelson

Atriplex spatiosa A. Nelson = Atriplex rosea L .

Atriplex triangulais Willd. $=$ Atriplex prostrata

Boucher ex DC. in Lam. \& DC.

Atropa pysalodes L. = Nicandra physalodes (L.) Gaertn.
AUREOLARIA Raf. (Scrophulariaceae)

A. flava (L.) Farw., Yellow False Foxglove var. flava

= A. glauca (Eddy) Raf.

$=$ Gerardia flava $\mathrm{L}$.

$=$ G. integrifolia A. Gray

$=\mathrm{G}$. quercifolia Pursh

var. macrantha Pennell, Large-flowered False Foxglove

$=$ Gerardia flava var. macrantha (Pennell) Fern.

A. pedicularia (L.) Raf., Lousewort False Foxglove, Cutleaf False-Foxglove $=$ A. pedicularia var. intercedens Pennell $=$ Gerardia pedicularia L.

Aureolaria pedicularia var. intercedens Pennell $=$ Aureolaria pedicularia (L.) Raf.

A. virginica (L.) Pennell, Downy False Foxglove $=$ Gerardia virginica (L.) BSP.

AVENA L. (Poaceae)

*A. fatua L., Oats

*ssp. fatua, Wild Oats

*ssp. sativa (L.) Thell.

= A. sativa $\mathrm{L}$.

Avena sativa L. = Avena fatua L. ssp. sativa (L.) Thell.

Avena striata (of authors) $=$ Schizachne purpurascens (Torr.) Swallen

Azalea nudiflora L. $=$ Rhododendron periclymenoides (Michx.) Shinners

Azalea periclymenoides Michx. = Rhododendron periclymenoides (Michx.) Shinners

AZOLLA Lam. (Azollaceae)

Azolla filiculoides of NY reports, not Lam. ex Lam. et al. = Azolla caroliniana Willd.

A. caroliniana Willd., Mosquito-fern, Water-fern $=$ Azolla filiculoides of NY reports, not Lam. ex Lam. et al.

BALSAMITA Mill. (Asteraceae)

*B. major Desf., Costmary, Mint-Geranium $=\mathrm{B}$. vulgaris Willd.

= Chrysanthemum balsamita (L.) Baill.

$=\mathrm{C}$. balsamita var. tanacetoides Boiss

$=$ Tanacetum balsamita L.

Balsamita vulgaris Willd. = Balsamita major Desf . 
BAPTISIA L. (Fabaceae)

B. lactea (Raf.) Thieret, Prairie False-Indigo $=$ B. leucantha Torr. \& A. Gray

Baptisia leucantha Torr. \& A. Gray = Baptisia lactea (Raf.) Thieret

B. tinctoria (L.) Vent., Wild Indigo, Rattleweed, Horsefly-weed

$=\mathrm{B}$. tinctoria var. crebra Fern.

$=$ B. tinctoria var. projecta Fern.

$=$ Sophora tinctoria L.

BARBAREA R.Br. ex Ait. (Brassicaceae)

*B. verna (Mill.) Aschers, Early Wintercress, Winter Belle-Isle, Land-Cress

= Campe verna (Mill.) Heller

$=$ Erysimum vernum Mill.

*B. vulgaris R. Br., Winter Cress, Yellow Rocket, Creesy-Greens

= B. stricta Andrz. ex Bess.

$=$ B. taurica DC.

$=$ B. vulgaris var. arcuata $($ Opiz \& Presl $)$ Fries

$=\mathrm{B}$. vulgaris var. stricta A. Gray

= Campe barbarea (L.) Wight

$=$ C. barbarea (L.) Wight var. hirsuta

(Weihe.) House

= C. stricta (Andrz. ex Bess.) Wight

$=$ Erysimum barbarea L.

BARTONIA Muhl. ex Willd. (Gentianaceae)

B. virginica (L.) BSP., Yellow Bartonia, Screwstem

BASSIA All. (Chenopodiaceae)

*B. scoparia (L.) A. Scott, Kochia, Summer Cypress, Mexican Fireweed

= Kochia scoparia (L.) Roth ex Schrad.

Batrachium longirostre (Godr.) F. Schultz = Ranunculus longirostris Godr.

Batrachium trichophyllum (Chaix ex Vill.) Bosch. $=$ Ranunculus trichophyllus Chaix ex Vill.

BECKMANNIA Host (Poaceae)

Beckmannia eruciformis (of American authors) $=$ Beckmannia syzigachne ssp. baicalensis (Kusn.) Koy. \& Kaw.

*B. syzigachne (Steud.) Fern. Beckman's Grass, Slough-Grass ssp. baicalensis (Kusn.) Koy. \& Kaw. $=$ B. eruciformis $($ L. $)$ Host

BELLIS L. (Asteraceae)

*B. perennis L., English Daisy, Lawn Daisy

Benzoin aestivale (L.) Nees = Lindera benzoin (L.) Blume

Benzoin odoriferum Nees \& ed 2 of Gray's Manual $=$ Lindera benzoin $(\mathrm{L}$.) Blume

BERBERIS L. (Berberidaceae)

Berberis aquilifolium Pursh. $=$ Mahonia aquilifolium (Pursh) Nutt.

*B. thunbergii DC., Japanese Barberry

*B. vulgaris L., Common Barberry

BERTEROA DC. (Brassicaceae)

*B. incana (L.) DC., Hoary Alyssum

$=$ Alyssum incanum L.

= Berteroa obliqua of NY reports, not (Sibth. \& Sm.) DC.

$=$ B. mutabilis of NY reports, not (Vent.) DC.

BERULA Koch (Apiaceae)

B. erecta (Huds.) Cov., Wild Parsnip, Waterparsnip

$=$ B. erecta (Huds.) Cov. var. incisa (Torr.) Cronq.

$=$ B. pusilla (Nutt.) Fern.

$=\mathrm{B}$. angustifolia (of American authors)

BETULA L. (Betulaceae)

Betula alba of authors in part = Betula pubescens Ehrh.

Betula alba var. populifolia (Marsh.) Spach = Betula populifolia Marsh.

B. alleghaniensis Britt., Yellow Birch

$=$ B. alleghanensis ("corrected spelling by Britton in Britt. \& Brown, 1903"), Mitchell \& Tucker 1997.

$=$ B. alleghaniensis var. macrolepis (Fern.) Brayshaw $=$ B. carpinifolia Michx.

$=$ B. excelsa Poir

$=\mathrm{B}$. lutea Michx. f.

$=\mathrm{B}$. lutea var. alleghaniensis (Britt.) Ashe

Betula carpinifolia Michx. $=$ Betula alleghaniensis Britt.

Betula excelsa Poir $=$ Betula alleghaniensis Britt. 
Betula glandulosa var. glandulifera (Regel)

Gleason $=$ Betula pumila L .

B. lenta L., Black Birch, Sweet Birch, Cherry Birch

Betula lutea Michx.f. = Betula alleghaniensis Britt.

Betula lutea Michx. f. var. alleghaniensis (Britt.) Ashe $=$ Betula alleghaniensis Britt.

B. nigra L., River Birch, Red Birch

Betula odorata Bechst. $=$ Betula pubescens Ehrh.

B. papyrifera Marsh., Paper Birch, Canoe Birch, White Birch 3

= B. papyrifera var. commutata (Regel) Fern.

$=$ B. papyrifera var. pensilis Fern.

B. populifolia Marsh., Gray Birch, Gire Birch, Old Field Birch, White Birch

$=$ B. alba var. populifolia (Marsh.) Spach

*B. pubescens Ehrh., Downy Birch

$=\mathrm{B}$. alba of authors in part

$=$ B. odorata Bechst.

B. pumila L., Swamp Birch

$=$ B. glandulosa var. glandulifera (Regel) Gleason

$=$ B. pumila var. glandulifera Regel

BIDENS L. (Asteraceae)

*B. aristosa (Michx.) Britt., Western Tickseed, Beggar-Ticks, Sticktights

$=$ B. aristosa var. retrorsa (Sherff) Wund.

$=$ B. polylepis Blake

Bidens beckii Torr. ex Spreng. = Megalodonta beckii (Torr. ex Spreng.) Greene

*B. bipinnata L., Spanish Needles

B. cernua L., Nodding Sticktight, Bur-Marigold $=\mathrm{B}$. cernua varieties

Bidens chrysanthemoides Michx. $=$ Bidens laevis (L.) BSP

Bidens comosa (Gray) Wieg. = Bidens tripartita L.

B. connata Muhl. ex Willd., Beggar-ticks, Sticktights

= B. connata var. petiolata (Nutt.) Farw.

B. coronata (L.) Britt., Tickseed Sunflower

$=\mathrm{B}$. coronata varieties

= B. coronata var. trichosperma (Michx.) Fern.

$=\mathrm{B}$. trichosperma (Michx.) Britt.

$=\mathrm{B}$. trichosperma var. tenuiloba (A. Gray) Britt.

$=$ Coreopsis trichosperma Michx.

B. frondosa L., Common Beggar-ticks
$=\mathrm{B}$. frondosa varieties

B. laevis (L.) BSP., Smooth Beggar-ticks

$=\mathrm{B}$. chrysanthemoides Michx.

Bidens polylepis Blake $=$ Bidens aristosa $($ Michx . Britt.

Bidens trichosperma (Michx.) Britt. = Bidens coronata (L.) Britt.

Bidens trichosperma var. tenuiloba (A. Gray) Britt. $=$ Bidens coronata $(\mathrm{L}$.$) Britt.$

B. tripartita L., Swamp Beggar-ticks $=$ B. $\operatorname{comosa}$ (A. Gray) Wieg.

B. vulgata Greene, Tall Beggar-ticks

Bilderdykia cilinode $($ Michx. $)$ Greene $=$ Polygonum cilinode Michx.

Bilderdykia convolvulus (L.) Dumort. = Polygonum convolvulus L.

Blechnum virginicum L. = Woodwardia virginica (L.) $\mathrm{Sm}$.

Blephariglottis blephariglottis (Willd.) Rydb. ex Britt. = Platanthera blephariglottis (Willd.) Lindl.

Blephariglottis grandiflora (Bigel.) Rydb. ex Britt. = Platanthera grandiflora (Bigel.) Lindl.

Blephariglottis leucophaea (Nutt.) Farw. = Platanthera leucophaea (Nutt.) Lindl.

Blephariglottis psycodes (L.) Rybd. ex Britt. = Platanthera psycodes (L.) Lindl.

BLEPHILIA Raf. (Lamiaceae)

B. hirsuta (Pursh) Benth., Wood Mint

Blitum bonus-henricus Reichenb. $=$ Chenopodium bonus-henricus L.

Blitum capitatum L. $=$ Chenopodium capitatum (L.) Aschers.

Blitum maritimum Nutt. $=$ Chenopodium rubrum L.

Boebera papposa (Vent.) Hitchc. = Dyssodia papposa (Vent.) Hitchc.

Boechera canadensis (L.) Al-Shehbaz $=$ Arabis canadensis L.

Boechera drummondii (A. Gray) Á. \& D. Löve = Arabis drummondii A. Gray

Boerchera laevigata (Muhl. ex Willd.) Al-Shehbaz = Arabis laevigata (Muhl. ex Willd.) Poir. ex Lam.

BOEHMERIA Jacq. (Urticaceae)

B. cylindrica (L.) Sw., False Nettle 
$=\mathrm{B}$. cylindrica var. drummondiana (Wedd.)

Wedd.

Bolboschoenus fluviatilis (Torr.) Soják = Scirpus fluviatilis (Torrey) A. Gray

Bolboschoenus maritimus (L.) Palla ssp. paludosus (A. Nelson) T. Koyama $=$ Scirpus maritimus L.

BORAGO L. (Boraginaceae)

*B. officinalis L., Borage

BOTRYCHIUM Sw. (Ophioglossaceae)

Botrychium ternatum var. lunarioides (Michx.)

D.C. Eat. $=$ Botrychium lunarioides

(Michx.) Sw. (excluded by Zander \& Pierce 1979)

Botrychium angustisegmentum (Pease \& Moore) Fern. = Botrychium lanceolatum var. angustisegmentum Pease \& Moore

B. dissectum Spreng., Dissected Grape-fern, Cutleaf Grape-fern; see note below under Botrychium lunarioides.

$=$ B. dissectum var. elongatum Gilb. \& Haberer

$=\mathrm{B}$. dissectum var. obliquum (Muhl. ex Willd.) Clute

= B. ternatum Sw. var. dissectum (Spreng.)

D. Eaton

$=$ B. obliquum Muhl.

= B. obliquum var. elongatum Gilbert $\&$ Haberer

= B. obliquum var. dissectum (Spreng.) Prantl

Botrychium dissectum $\times$ multifidum $=$ Botrychium oneidense (Gilbert) House

Botrychium dissectum var. obliquum (Muhl. ex Willd.) Clute $=$ Botrychium dissectum Spreng.

Botrychium dissectum var. oneidense (Gilb.)

CLute $=$ Botychium oneidense (Gilb.)

House

B. lanceolatum (Gmel.) Aongstr., Lance-leaved Grape Fern

var. angustisegmentum Pease \& Moore

$=$ Botrychium angustisegmentum (Pease \& Moore) Fern.

B. lunaria (L.) Sw., Moonwort $=\mathrm{B}$. lunaria var. onondagense (Underw.) House
$=\mathrm{B}$. onondagense Underw.

Botrychium lunaria var. onondagense (Underw.) House $=$ Botrychium lunaria (L.) Sw. Botrychium matricariae (Schrank) Spreng. = Botrychium multifidum (Gmel.) Rupr.

Botrychium lunarioides Swartz of Gray's 5th edition of the manual has two varieties: var. obliquum (= Botychium obliquum Muhl.) and var. dissectum (= Botrychium dissectum Muhl.) (p.672). George Clinton used these names in his collecting diary. These ferns today are probably Botrychium dissectum Spreng.

B. matricariifolium (A.Br. ex Doell) A. Br. ex Koch, Matricary Grape-fern, Daisy-Leaf Grape-fern, Chamomile Grape-fern $=\mathrm{B}$. neglectum Wood

B. multifidum (Gmel.) Rupr., Intermediate Leather Grape Fern, Leather-leaved Grapefern

= B. matricariae (Schrank) Spreng.

$=$ B. multifidum var. intermedium (D. Eat.) Farw.

$=$ B. silaifolium Presl

= B. ternatum var. intermedium D.C. Eat.

Botrychium multifidum var. oneidense (Gilb.) Farw. $=$ Botrychium oneidense (Gilbert) House

Botrychium neglectum Wood = Botrychium matricariifolium (A.Br. ex Doell) A. Br. ex Koch

Botrychium obliquum Muhl. = Botrychium dissectum Spreng.

Botrychium obliquum var. dissectum (Spreng.) Prantl $=$ Botrychium dissectum Spreng.

Botrychium obliquum var. elongatum Gilbert \& Haberer $=$ Botrychium dissectum Spreng.

Botrychium obliquum var. oneidense (Gilbert) Waters $=$ Botrychium oneidense (Gilbert) Waters

B. oneidense (Gilbert) House, Oneida Grape Fern $=$ B. dissectum var. oneidense (Gilb.) Clute

$=\mathrm{B}$. dissectum $\times$ multifidum

= B. multifidum var. oneidense (Gilb.) Farw.

= B. obliquum var. oneidense (Gilbert) Waters

Botrychium onondagense Underw. $=$ Botrychium lunaria (L.) Sw. 
Botrychium silaifolium Presl $=$ Botrychium multifidum (Gmel.) Rupr.

B. simplex E. Hitchc., Little Grape Fern, Least Moonwort, Dwarf Grape-fern

= B. simplex var. laxifolium Clausen, not (Clausen) Fern.

$=$ B. simplex var. tenebrosum (A. Eaton) Clausen

$=\mathrm{B}$. tenebrosum A. Eaton

Botrychium tenebrosum A. Eaton = Botrychium simplex E. Hitchc.

Botrychium ternatum var. dissectum (Spreng) D.C. Eat. $=$ Botrychium dissectum Spreng.

Botrychium ternatum var. intermedium D.C. Eat. = Botrychium multifidum (Gmel.) Rupr.

B. virginianum (L.) Sw., Rattlesnake Fern

BRACHYACTIS Ledeb. (Asteraceae)

*B. ciliata (Ledeb.) Ledeb., Rayless Aster ssp. angusta (Lindl.) A. Jones

$=$ Aster brachyactis Blake

$=$ Brachyactis angusta (Lindl.) Britt.

= Symphyotrichum ciliatum (Ledeb.) Nesom

BRACHYELYTRUM Beauv. (Poaceae)

Brachyelytrum aristatum (Pers.) R.\& S. = Brachyelytrum erectum (Schreb.) Beauv.

Brachyelytrum aristatum Beauv. of Gray's 5th ed.

$=$ Brachyelytrum erectum (Schreb.) Beauv.

Brachyelytrum aristosum (Michx.) Trel. =

Brachyelytrum erectum (Schreb.) Beauv.

B. erectum (Schreb.) Beauv., Bearded Short-husk,

Brachyelytrum

$=$ Dilepyrum erectum (Schreb.) Farw.

$=$ Muhlenbergia erecta Schreb. in Roth ex

Spreng.

$=$ Brachyelytrum aristatum (Pers.) R.\& S.

$=$ B. aristatum Beauv. of Gray's 5th ed

$=\mathrm{B}$. $\operatorname{aristosum}($ Michx.) Trel.

Brachyelytrum erectum var. glabratum (Vasey ex

Millsp.) T. Koyama \& Kawano =

Brachyelytrum septentrionale (Babel) G.

Tucker

Brachyelytrum erectum var. septentrionale Babel =

Brachyelytrum septentrionale (Babel) G.

Tucker

B. septentrionale (Babel) G. Tucker, Northern

Shorthusk

$=\mathrm{B}$. erectum var. septentrionale Babel
= B. erectum var. glabratum (Vasey ex

Millsp.) T. Koyama \& Kawano

BRASENIA Schreb. (Cabombaceae)

Brasenia peltata Pursh = Brasenia schreberi Gmel.

B. schreberi Gmel., Water-shield, Purple Wen-

dock

$=$ B. peltata Pursh

BRASSICA L. (Brassicaceae)

Brassica arvensis (L.) Rabenh. $=$ Sinapis arvensis L.

Brassica arvensis var. schkuhriana (Reichenb.) Wheeler $=$ Sinapis arvensis L.

Brassica campestris L. = Brassica rapa L.

Brassica eruca L. = Eruca vesicaria (L.) Cav. ssp. sativa (Mill.) Thell.

*B. juncea (L.) Czern., Indian Mustard, Brown Mustard, Chinese Mustard, Curly Mustard

$=$ B. japonica Thunb.

$=\mathrm{B}$. juncea varieties

Brassica kaber (DC.) L. Wheeler $=$ Sinapis arvensis L.

Brassica kaber var. pinnatifida (Stokes) Wheeler $=$ Sinapis arvensis L.

*B. nigra (L.) Koch, Black Mustard $=$ Sinapis nigra $\mathrm{L}$.

*B. oleracea L., Cabbage, Kale, Broccoli, Cauliflower, Kohlrabi, Brussels Sprouts $=$ B. sylvestris $(\mathrm{L}$.) Mill.

*B. rapa L., Field Mustard, Turnip, Bok Choy, Chinese Cabbage

$=$ B. campestris $\mathrm{L}$

= B. rapa ssp. oleifera DC.

Brassica sinapistrum Boiss. $=$ Sinapis arvensis L.

Brassica sylvestris (L.) Mill. = Brassica oleracea L.

BRIZA L. (Poaceae)

*B. media L., Quaking Grass

Bromelica striata (Michx.) Farw. $=$ Schizachne purpurascens (Torr.) Swallen

BROMUS L. (Poaceae)

Bromus altissimus Pursh $=$ Bromus latiglumis (Shear) Hitchc.

B. ciliatus L., Fringed Brome-grass

Bromus ciliatus var. purgans (L.) Gray = Bromus pubescens Muhl. ex Willd. 
*B. commutatus Schrad., Fringed Brome, Hairy Chess

*B. erectus Huds., Upright Brome-grass, Hungarian Brome

*B. hordeaceus L., Soft Chess

$=\mathrm{B}$. mollis of authors, not L.

Bromus incanus (Shear) Hitchc. $=$ Bromus latiglumis (Shear) Hitchc.

*B. inermis Leyss., Hungarian Brome-grass, Smooth Brome, Awnless Brome $=\mathrm{B}$. inermis var. divaricatus Rohl.

*B. japonicus Thunb. ex Murr., Japanese Bromegrass, Japanese Chess

B. kalmii Gray, Kalm's Brome-grass

B. latiglumis (Shear) Hitchc., Canada Brome, Tall Wood-chess

$=$ Bromus altissimus Pursh

$=$ Bromus incanus (Shear) Hitchc.

Bromus mollis of authors, not L. $=$ Bromus hordeaceus L.

Bromus nottowayanus Fern. reported for southern Ontario by Wagnon (1952) "with mapped records from the Niagara Peninsula [Regional Municipality of Niagara] and at the eastern end of Lake Ontario (probably within New York State)" is said to be closely related to B. pubescens, the sheaths densely pubescent at the summit, "but has a 5-nerved upper glume and may be the result of hybridization of that species with B. altissimus or even B. kalmii" (Dore \& McNeill 1980). Morton and Venn (1990) indicate that B. nottowayanus is probably B. pubescens as does Voss (1972). Not noted for New York State by Mitchell and Tucker (1997).

*B. pubescens Muhl. ex Willd., Canada Brome Grass

$=$ B. ciliatus var. purgans (L.) Gray

$=\mathrm{B}$. purgans of authors, not L.

Bromus purgans L. $=$ Bromus pubescens Muhl. ex Willd.

*B. racemosus L., Smooth Brome-grass, Soft Chess

*B. secalinus L., Cheat, Chess, Rye-brome

*B. sterilis L., Barren Brome-grass, Povertybrome

*B. tectorum L., Downy Brome-grass, Cheatgrass
Brunella (orth. var.) $=$ Prunella L.

BRUNNERA Stev. (Boraginaceae)

*B. macrophylla (Adams) I. M. Johnston, Siberian Bugloss, Garden Forget-me-not $=$ Anchusa myosotidiflora Lehm. = Myosotis macrophylla Adams

BUCHNERA L. (Scrophulariaceae)

B. americana L., Bluehearts

BUDDLEJA L. (Buddlejaceae)

*B. davidii Franch., Summer Lilac, Butterflybush, Buddleja

BUGLOSSOIDES Moench (Boraginaceae)

*B. arvense (L.) Johnst., Corn Gromwell, Bastard Alkanet

$=$ Lithospermum arvense $\mathrm{L}$.

BULBOSTYLIS Kunth (Cyperaceae)

B. capillaris (L.) Clarke, Sand-rush

$=$ Fimbristylis capillaris A. Gray

$=$ Stenophyllus capillaris (L.) Britt.

$=\mathrm{S}$. capillaris var. cryptostachys Fern.

Bursa bursa-pastoris (L.) Britt. = Capsella bursapastoris (L.) Medik.

BUTOMUS L. (Butomaceae)

*B. umbellatus L., Flowering Rush

CACALIA L. (Asteraceae)

C. atriplicifolia L., Orache-leaved Indian-plantain, Pale Indian-plantain, Wild Collard

= Arnoglossum atriplicifolium (L.) H. Robinson

C. suaveolens L., Indian Plantain, Sweet-scented Indian-plantain

CAKILE Mill. (Brassicaceae)

Cakile americana Nutt. (of local authors) $=$ Cakile edentula (Bigel.) Hook. ssp. edentula

C. edentula (Bigel.) Hook., Sea-rocket, Shorerocket

ssp. edentula

ssp. lacustris Fern.

= C. edentula ssp. lacustris (Fern.) Hultén

CALAMAGROSTIS Adans. (Poaceae) 
Calamagrostis arenaria Roth. of Gray's Manual ed. 5 = Ammophila breviligulata Fernald, q.v.

C. canadensis (Michx.) Beauv., Bluejoint Grass, Canada Bluejoint Grass

var. canadensis

$=\mathrm{C}$. canadensis var. robusta Vasey

$=\mathrm{C}$. hyperborea Lange

Calamagrostis hyperborea Lange $=$ Calamagrostis canadensis (Michx.) Beauv. var. canadensis

CALAMINTHA Mill. (Lamiaceae)

C. arkansana (Nutt.) Shinners, Low Calamint, Calamint

$=\mathrm{C}$. nuttallii Benth.

$=$ Clinopodium arkansanum (Nutt.) House

$=$ Hedeoma arkansana Nutt.

$=$ Micromeria glabella of Ontario reports, not of Benth.

$=$ Satureja arkansana (Nutt.) Briq.

= S. glabella var. angustifolia (Torr.) Svenson

$=\mathrm{S}$. glabella of Ontario reports, not of

(Michaux) Briq.

$=$ S. glabra (Nutt.) Fern.

$=$ Calamintha glabella var. nuttallii (Benth.) Gray

Calamintha clinopodium Benth. in DC. = Clinopodium vulgare $\mathrm{L}$.

Calamintha glabella var. nuttallii (Benth.) Gray = Calamintha arkansana (Nutt.) Shinners

Calamintha nuttallii Benth. $=$ Calamintha arkansana (Nutt.) Shinners

CALAMOVILFA (A. Gray) Hack. ex Scribn. \& Southw. (Poaceae)

*C. Iongifolia (Hook.) Scribn., Great Lakes

Sandreed, Dunegrass

var. magna Scribn. \& Merr.

CALLA L. (Araceae)

C. palustris L., Wild Calla, Water-arum

CALLITRICHE L. (Callitrichaceae)

Callitriche anceps Fern. $=$ Callitriche heterophylla Pursh

C. heterophylla Pursh, Large Water-starwort $=\mathrm{C}$. anceps Fern.

C. palustris L., Water-starwort $=\mathrm{C}$. verna $\mathrm{L}$.
Callitriche verna L. $=$ Callitriche palustris L.

CALOPOGON R. Br. ex Ait. (Orchidaceae) Calopogon pulchellus (Salisb.) R. Br. ex Ait. = Calopogon tuberosus (L.) BSP.

C. tuberosus (L.) BSP., Grass Pink Orchid = C. pulchellus (Salisb.) R. Br. ex Ait. $=$ Calthea pulchella (Salisb.) Salisb., not (Willd.) Salisb.

CALTHA L. (Ranunculaceae)

C. palustris L., Marsh Marigold, Cowslip

$=$ C. flabellifolia Pursh

= C. palustris var. flabellifolia (Pursh) Torr.

\& A. Gray

= C. palustris var. radicans (Forst.) Hartm.

$=\mathrm{C}$. radicans Forst.

Calthea pulchella (Salisb.) Salisb., not (Willd.) Salisb. = Calopogon tuberosus (L.) BSP.

CALYPSO Salisb. (Orchidaceae)

Calypso borealis Salisb. $=$ Calypso bulbosa (L.) Oakes

C. bulbosa (L.) Oakes, Calypso Orchid, Fairyslipper

$=$ C. borealis Salisb.

$=$ Cytherea bulbosa (L.) Salisb.

CALYSTEGIA R. Br. (Convolvulaceae)

Calystegia pellita (Ledeb.) G. Don = Calystegia hederacea Wallich

*C. hederacea Wallich, Japanese Bindweed $=$ Calystegia pellita (Ledeb.) G. Don

$=\mathrm{C}$. pubescens Lindl.

$=$ Convolvulus pellitus Ledeb.

$=\mathrm{C}$. japonicus Thunb.

$=$ C. wallichianus Spreng.

Calystegia pubescens Lindl. $=$ Calystegia hederacea Wallich

C. sepium (L.) R. Br., Hedge-bindweed, Wild Morning-glory, Trailing Hedge-bindweed

*ssp. sepium (L.) R. Br. $=$ Convolvulus nashii House

$=$ C. sepium var. communis R. Tryon

= C. sepium var. sepium

C. spithamea (L.) Pursh, Low Bindweed $=$ Convolvulus spithameus $\mathrm{L}$.

CAMELINA Crantz (Brassicaceae) 
*C. microcarpa Andrz. ex DC., Small-fruited False Flax, Littlepod

*C. sativa (L.) Crantz, False Flax, Dutch Flax, Gold-of-Pleasure

CAMPANULA L. (Campanulaceae)

C. americana L., Tall Bellflower = Campanulastrum americanum (L.) Small

C. aparinoides Pursh, Marsh Bellflower

$=\mathrm{C}$. aparinoides var. grandiflora $\mathrm{Holz}$

= C. aparinoides var. uliginosa (Rydb.)

Gleason

$=$ C. uliginosa Rydb.

*Campanula glomerata L., a rare introduction in

New York state is excluded from our flora by Zander and Pierce (1979).

*C. rapunculoides L., Creeping Bellflower $=$ C. rapunculoides var. ucranica (Bess.) Koch

C. rotundifolia L., Harebell, Bluebell

Campanula uliginosa Rydb. = Campanula aparinoides Pursh

Campanulastrum americanum (L.) Small = Campanula americana L.

Campe barbarea (L.) Wight $=$ Barbarea vulgaris R . $\mathrm{Br}$.

Campe barbarea (L.) Wight var. hirsuta (Weihe.) House $=$ Barbarea vulgaris $\mathrm{R}$. Br.

Campe stricta (Andrz. ex Bess.) Wight $=$ Barbarea vulgaris $\mathrm{R}$. Br.

Campe verna (Mill.) Heller = Barbarea verna (Mill.) Aschers

[CAMPSIS Lour. (Bignoniaceae)]

[C. radicans (L.) Seem. ex Bureau, Trumpetcreeper, Trumpet-vine, Trumpet-flower, possibly est. in Regl. M. Niag. Oldham 1999]

Camptosorus rhizophyllus (L.) Link $=$ Asplenium rhizophyllum L.

CANNABIS L. (Cannabaceae)

Cannabis indica Lam. = Cannabis sativa L.

*C. sativa L., Hemp, Marijuana

$=$ C. indica Lam.

= C. sativa ssp. indica (Lam.) Small \& Cronq.

Capnoides flavula (Raf. ex Desv.) Kuntze = Corydalis flavula (Raf. ex Desv.) DC.
Capnoides sempervirens (L.) Borck. ex Roemer = Corydalis sempervirens (L.) Pers.

CAPSELLA Medik. (Brassicaceae)

*C. bursa-pastoris (L.) Medik., Shepherd's Purse, Pick-pocket

$=$ Bursa bursa-pastoris (L.) Britt.

= Capsella bursa-pastoris var. bifida Crépin

$=$ Thlaspi bursa-pastoris L.

Carara didyma (L.) Britt. $=$ Coronopus didymus

(L.) $\mathrm{Sm}$.

CARDAMINE L. (Brassicaceae)

Cardamine arenicola Britt. $=$ Cardamine parviflora L. var. arenicola (Britt.) Schulz

C. bulbosa (Schreb. ex Muhl.) BSP, Bulbous Spring Cress

= Arabis bulbosa Schreb. ex Muhl.

$=$ A. rhomboidea Pers.

= Cardamine rhomboidea (Pers.) DC.

C. concatenata (Michx.) Schwein., Cut-leaved Toothwort

$=$ Dentaria concatenata Michx.

= D. laciniata Muhl. ex Willd.

C. concatenata $\times$ diphylla $=\mathbf{C} . \times$ maxima (Nutt.)

Wood, Large Toothwort

$=$ Cardamine maxima (Nutt.) Wood

$=$ Dentaria diphylla $\times$ laciniata

$=$ D. diphylla $\times$ maxima

$=$ D. $\times$ incisa K. Schum.

$=$ D. $\times$ incisifolia Eames

$=$ D. laciniata $\times$ maxima

$=$ D. maxima Nutt.

C. diphylla (Michx.) Wood, Two-leaved

Toothwort

$=$ Dentaria diphylla Michx.

C. douglassii Britt. (not Torr.), Purple Spring-cress $=$ C. rhomboidea var. purpurea Torr.

*C. flexuosa With., Rock-cress

Cardamine flexuosa of some NY reports, not With. $=$ Cardamine parviflora L. var. arenicola (Britt.) Schulz OR Cardamine pensylvanica Muhl. ex Willd.

*Cardamine hirsuta L. in New York is a rare introduction, excluded by Zander and Pierce (1979).

Cardamine hirsuta sensu Torrey's NY Flora, not L. = Cardamine pensylvanica Muhl. ex Willd. 
Cardamine maxima (Nutt.) Wood $=[$ Cardamine concatenata $\times$ diphylla $=$ C. $\times$ maxima (Nutt.) Wood]

Cardamine parviflora (of American authors) $=$ Cardamine parviflora var. arenicola (Britt.) O. E. Schulz

C. parviflora L., Small-flowered Bitter Cress, Small-flowered Bittercress var. arenicola (Britt.) O.E. Schulz

$=\mathrm{C}$. arenicola Britt.

$=\mathrm{C}$. flexuosa of some NY reports, not With.

$=\mathrm{C}$. parviflora var. parviflora (of American authors)

$=\mathrm{C}$. virginica Michx.

C. pensylvanica Muhl. ex Willd., Pennsylvania Bitter Cress

$=\mathrm{C}$. flexuosa of some NY reports, not With.

$=$ C. hirsuta sensu Torrey's NY Flora, not L.

$=\mathrm{C}$. pensylvanica var. brittoniana Farw.

Cardamine pensylvanica var. brittoniana Farw. = Cardamine pensylvanica Muhl. ex Willd.

C. pratensis L., Cuckoo-flower = C. palustris Peterm. ex Rabenh.

$=$ C. pratensis var. palustris Wimm. \& Graeb.

$=$ C. pratensis ssp. palustris (Wimm. \& Graebn.) Clausen

Cardamine rhomboidea DC.$=$ Cardamine bulbosa (Schreb. ex Muhl.) BSP

Cardamine rhomboidea var. purpurea Torr. $=$ Cardamine douglassii Britt. (not Torr.)

C. rotundifolia Michx., Round-leaved Cress Cardamine spathulata Michx. = Arabis lyrata L.

Cardamine virginica Michx. $=$ Cardamine parviflora L. var. arenicola (Britt.) Schulz

Cardaminopsis lyrata (L.) Hiitonen. = Arabis lyrata L.

CARDARIA Desv. (Brassicaceae)

*Cardaria draba (L.) Desv., Hoary Cress $=$ Lepidium draba $\mathrm{L}$.

CARDUUS L. (Asteraceae)

*C. acanthoides L., Bristle-thistle, Spiny Plumeless-thistle

Carduus mariana L. = Silybum marianum (L.) Gaertn.

*C. nutans L., Nodding Thistle, Musk-thistle

CAREX L. (Cyperaceae)
Carex abacta Bailey $=$ Carex michauxiana Boeckl.

Carex aenea Fern. $=$ Carex argyrantha Tuckerm

C. aestivalis Curtis ex A. Gray, Summer-sedge

C. alata Torr., Broad-winged Sedge

C. albicans Willd. ex Spreng., Sedge var. albicans

= C. artitecta Mackz.

$=\mathrm{C}$. nigromarginata var. muhlenbergii (A. Gray) Gleason

$=\mathrm{C}$. varia of old NY reports, not Muhl. ex Willd.

var. emmonsii (Dewey ex Torrey) Rettig, Emmons' Sedge

$=\mathrm{C}$. emmonsii Dewey ex Torrey

C. albursina Sheldon, White Bear Sedge $=\mathrm{C}$. laxiflora var. latifolia Boott

C. alopecoidea Tuckerm., Fox-sedge

C. amphibola Steud. var. turgida Fern., Gray Sedge

$=\mathrm{C}$. grisea Wahl.

$=\mathrm{C}$. grisea auct. non Wahlenb.

$=\mathrm{C}$. corrugata Fern.

Carex anceps Muhl. $=$ Carex laxiflora Lam. var. laxiflora

Carex angustata Boott, according to Gray's Manual, ed. 5, $1867=$ Carex stricta Lam.

Carex angustior Mackenzie ex Rydb. = Carex echinata Murr.

C. annectens (Bickn.) Bickn.

var. annectens

var. xanthocarpa (Bickn.) Wieg. $=\mathrm{C}$. brachyglossa Mackenzie

C. appalachica Webber \& Ball

= C. radiata (Wahlenberg) Small, "formerly

C. radiata sensu Mackenzie"

$=\mathrm{C}$. rosea var. radiata (Wahlenberg) Dewey

C. aquatilis Wahl., Northern Water-sedge $=\mathrm{C}$. aquatilis var. altior (Rydb.) Fern.

$=$ C. substricta (Kuekenth) Mackenzie ex Rydb.

C. arctata Boott ex Hook., Drooping Wood-sedge

C. argyrantha Tuckerm., Hay-sedge = C. aenea Fern. (sec. NYFA July 2005)

$=\mathrm{C}$. foenea of some old NY reports, not Willd.

= C. siccata Dewey (sec. NYFA July 2005)

Carex artitecta Mackz. $=$ Carex albicans Willd. ex Spreng. var. albicans

Carex asa-grayi Bailey = Carex grayi Carey 
C. atherodes Spreng., Wheat-ear Sedge

C. atlantica Bailey ssp. atlantica, Prickly Bog-sedge

$=$ C. atlantica var. incomperta (Bickn.) Herm.

$=\mathrm{C}$. incomperta Bickn.

ssp. capillacea (Bailey) Reznicek, Howe's Sedge

$=\mathrm{C}$. howei Mackenzie

C. aurea Nutt., Golden-fruited Sedge

Carea aurea Nutt. in part $=$ Carex garberi Fern.

C. backii Boott, Back's Sedge

$=$ C. durifolia Bailey

C. bebbii (Bailey) Olney ex Fern., Bebb's Sedge

Carex bicolor (of authors) $=$ Carex garberi Fern .

C. blanda Dewey, Charming Sedge, Woodland Sedge

$=\mathrm{C}$. laxiflora var. blanda (Dewey) Boott

Carex brachyglossa Mackenzie $=$ Carex annectens

(Bickn.) Bickn. var. xanthocarpa (Bickn.) Wieg.

C. brevior (Dewey) Mackenzie ex Lunell, Common Fescue-sedge

Carex brevior (Dewey) Mackenzie in part $=$ Carex merritt-fernaldii Mackenzie

C. bromoides Schkuhr ex Willd., Brome-like Sedge

C. brunnescens (Pers.) Poir. ex Lam., Brownish

Sedge

var. brunnescens

C. buxbaumii Wahl., Brown Sedge

$=$ C. polygama Schk.

C. canescens L., Silvery Sedge

= C. canescens var. disjuncta Fern.

$=$ C . canescens var. subloliacea Laest.

= C. curta Good., according to Gray, 1967,

Manual ed 5

C. careyana Torr. ex Dewey, Carey's Sedge

Carex cephalantha (Bailey) Bickn. $=$ Carex echinata Murr.

C. cephaloidea (Dewey) Dewey, Thin-leaved Sedge

$=$ C . sparganioides var. cephaloidea (Dewey) Carey

C. cephalophora Muhl. ex Willd., Southern Sedge, Ovel-leaved Sedge

Carex chlorophila Mackenzie $=$ Carex viridula Michx.

C. chordorrhiza Ehrh. ex L.f., Creeping Sedge

C. communis Bailey, Fibrous-rooted Sedge
C. comosa Boott, Bristly Sedge

C. complanata Torr. \& Hook., Northern Hirsute Sedge

$=$ C. hirsuta Willd., see Gray's Manual ed 5 p. 588

$=\mathrm{C}$. triceps (of authors)

C. conoidea Schkuhr ex Willd., Field-sedge

Carex convoluta Mackenzie $=$ Carex rosea Schkuhr ex Willd.

Carex corrugata Fern. $=$ Carex amphibola Steud. var. turgida Fern.

C. crawei Dewey ex Torr., Crawe's Sedge

C. crinita Lam., Sickle-sedge, Fringed Sedge

$=$ C. crinita var. brevicrinis Fern.

$=$ C. crinita var. minor Boott

$=$ C . crinita var. porteri (Olney) Fern.

$=$ C. crinita var. simulans Fern.

$=\mathrm{C}$. nigromarginata var. minor (Boott) Gleason

Carex crinita var. gynandra (Schwein.) Schwein. \& Torr. $=$ Carex gynandra Schwein .

Carex cristata $($ of authors $)=$ Carex cristatella Britt . ex Britt. \& Brown

C. cristatella Britt. ex Britt. \& Brown, Crested Sedge

$=$ C. cristata (of authors)

C. cryptolepis Mackenzie, Small Yellow Sedge

$=$ C. flava var. fertilis M. Peck

$=$ C. oederi Michx. according to House, 1924, p. 195

Carex curta Good., according to Gray, 1967, Manual ed $5=$ Carex canescens L.

C. debilis Michx., Rudge's Sedge, White-edge Sedge

var. rudgei Bailey = C. flexuosa Muhl. ex Willd.

C. deweyana Schwein., Dewey's Sedge

C. diandra Schrank, Lesser Panicled Sedge $=$ C. teretiuscula var. major Koch

C. digitalis Willd., Slender Wood-sedge

Carex dioica L. of authors in part $=$ Carex gynocrates Wormsk. ex Drej.

C. disperma Dewey, Soft-leaved Sedge $=$ C. tenella Schk.

Carex disticha (of Day, 1882) $=$ Carex sartwellii Dewey

Carex durifolia Bailey $=$ Carex backii Boott

C. eburnea Boott ex Hook., Bristle-leaved Sedge

C. echinata Murr., Prickly Sedge 
= C. angustior Mackenzie ex Rydb.

$=$ C. cephalantha (Bailey) Bickn.

=C. leersii Willd. of Zander \& Pierce (1979)

$=$ C. muricata of American authors, not L.

$=$ C. muricata L. var. angustata Carey

= C. muricata L. var. cephalantha (Bailey)

Wieg. \& Eames

Carex elachycarpa Fern. $=$ Carex sterilis Willd.

Carex emmonsii Dewey ex Torr. $=$ Carex albicans var. emmonsii (Dewey ex Torrey) Rettig.

C. emoryi Dewey ex Torrey, Emory's Sedge $=$ C. stricta var. elongata (Bock) Gleason

C. festucacea Schkuhr ex Willd., Fescue-sedge = C. straminea of some old NY reports, not Schkuhr ex Willd.

$=$ C. straminea Willd. var. festucacea Boott

Carex filiformis of authors, not L. $=$ Carex lasiocarpa ssp. americana (Fern.) Hultén

C. flaccosperma Dewey, Sedge var. glaucodea (Tuckerman ex Olney) Kuk., Blue-green Sedge

$=\mathrm{C}$. glaucodea Tuckerman ex Olney

Carex flaccidula Steudel, sec. Macdonald $1990=$ C. radiata (Wahl.) Small (not Dewey)

C. flava L., Yellow Sedge = C. flava var. laxior (Kuk.) Gleason $=$ C. laxior (Kuk.) Mackenzie

Carex flava var. fertilis Peck $=$ Carex cryptolepis Mackenzie

Carex flexuosa Muhl. ex Willd. $=$ Carex debilis Michx. var. rudgei Bailey

Carex foenea (of authors) $=$ Carex argyrantha Tuckerm.

C. folliculata L., Long Sedge

C. formosa Dewey, Handsome Sedge

C. garberi Fern., Garber's Sedge, Elk-sedge $=$ C. aurea Nutt. in part.

$=\mathrm{C}$. bicolor (of authors)

= C. garberi ssp. bifaria (Fern.) Hultén

$=\mathrm{C}$. hassei Bailey

Carex glaucodea Tuckerman ex Olney $=$ Carex flaccosperma Dewey var. glaucodea (Tuckerman ex Olney) Kuk.

C. gracilescens Steud.

C. gracillima Schwein., Graceful Sedge

C. granularis Muhl. ex Willd., Meadow-sedge var. granularis var. haleana (Olney) Porter $=\mathrm{C}$. shriveri Britt.
C. grayi Carey, Gray's Sedge

$=\mathrm{C}$. asa-grayi Bailey

$=$ C. asa-grayi Bailey var. hispidula (A. Gray)

Bailey

$=$ C. grayi var. hispidula A. Gray

Carex grayi var. hispidula A. Gray $=$ Carex grayi Carey

Carex grisea Wahl. $=$ Carex amphibola Steud. var. turgida Fern.

Carex gymnocrates of authors $=$ Carex gynocrates Wormsk. ex Drej.

C. gynandra Schwein., Nodding Sedge, Mountain Fringed Sedge

$=$ C . crinita var. gynandra (Schwein.)

Schwein. \& Torr.

C. gynocrates Wormsk. ex Drej., Northern Bogsedge

$=$ C. dioica L. of authors in part

$=\mathrm{C}$. gymnocrates of authors

Carex hassei Bailey $=$ Carex garberi Fern .

[C. haydenii Dewey, Hayden's Sedge Rarity Status: State; E TNC Rank: G5 S1: according to Young, (1998) this species is of possible occurrence in Niagara Co. due to unconfirmed report, oral, published or otherwise.]

Carex hirsuta Willd., see Gray's Manual ed p. 588 = Carex complanata Torr. \& Hook., q.v.

C. hirsutella Mackenzie, Hairy Green Sedge (Regl. M. Niag. Oldham 1999)

= Carex complanata Torr. \& Hook. var. hirsuta (Bailey) Gleason $=$ C. triceps Mackz.

C. hirtifolia Mackenzie, Pubescent Sedge $=\mathrm{C}$. pubescens Muhl.

C. hitchcockiana Dewey, Hitchcock's Sedge

Carex howei Mackenzie $=$ Carex atlantica Bailey ssp. capillacea (Bailey) Reznicek

[C. hyalinolepis Steud., Sedge There are unconfirmed reports of this species from Cattaraugus Co. (Young 1998).]

C. hystericina Muhl. ex Willd., Porcupine-sedge $=\mathrm{C}$. hystricina of authors

Carex hystricina of authors $=$ Carex hystericina Muhl. ex Willd.

Carex incomperta Bickn. $=$ Carex atlantica Bailey ssp. atlantica

C. interior Bailey, Inland Sedge 
C. intumescens Rudge, Bladder-sedge $=\mathrm{C}$. intumescens var. fernaldii Bailey

Carex irregularis Schwein. $=$ Carex viridula Michx.

C. jamesii Schwein., James' Sedge $=$ C. steudelii Kunth

C. lacustris Willd., Lake-bank Sedge $=\mathrm{C}$. riparia (of authors) $=$ C. riparia var. lacustris (Willd.) Kuekenth

C. laevivaginata (Kuekenth) Mackenzie ex Britt. \& Brown, Smooth-sheathed Sedge

C. lanuginosa Michx., Woolly Sedge $=\mathrm{C}$. lasiocarpa ssp. lanuginosa (Michx.) Clausen \& Wahl. = C. lasiocarpa var. latifolia (Boeck.) Gleason $=\mathrm{C}$. pellita Muhl.

C. lasiocarpa Ehrh., Slender Sedge ssp. americana (Fern.) Hultén

$=$ C. lasiocarpa var. americana Fern. $=\mathrm{C}$. filiformis auct. not of $\mathrm{L}$.

Carex lasiocarpa ssp. lanuginosa (Michx.) Clausen $\&$ Wahl. $=$ Carex lanuginosa Michx.

Carex lasiocarpa var. americana Fern. $=$ Carex lasiocarpa ssp. americana (Fern.) Hultén

Carex lasiocarpa var. latifolia (Boeck.) Gleason = Carex lanuginosa Michx.

C. laxiculmis Schwein., Spreading Sedge $=\mathrm{C}$. retrocurva Dewey in Wood

C. laxiflora Lam., Loose-flowered Sedge var. laxiflora

$=$ C. anceps Willd.

Carex laxiflora var. blanda (Dewey) Boott $=$ Carex blanda Dewey

Carex laxiflora var. latifolia Boott $=$ Carex albursina Sheldon

Carex laxior (Kuk.) Mackenzie $=$ Carex flava L.

Carex leersii Willd. of Zander \& Pierce (1979) = Carex echinata Murr.

C. leptalea Wahl., Bristle-stalked Sedge = C. polytrichoides Muhl. ex Willd.

C. leptonervia (Fern.) Fern., Northern Woodlandsedge

C. limosa L., Mud-sedge

C. lupuliformis Sartwell ex Dewey, Hop-like Sedge

C. Iupulina Muhl. ex Willd., Hop-sedge $=\mathrm{C} . \times$ macounii Dewey $=\mathrm{C}$. lupulina var. pedunculata Gray
Carex lupulina var. pedunculata Gray $=$ Carex lupulina Muhl. ex Willd.

C. lurida Wahl., Sallow Sedge = C. lurida var. exundans Bailey ex Britt. \& Brown

$=$ C. lurida var. pedunculata Gray

$=$ C. tentaculata Muhl. ex Willd.

Carex $\times$ macounii Dewey $=$ Carex lupulina Muhl . ex Willd.

C. meadii Dewey, Mead's Sedge

C. merritt-fernaldii Mackz., Fernald's Sedge $=\mathrm{C}$. brevior (Dewey) Mackz. in part.

C. michauxiana Boeckl., Michaux's Sedge $=$ C. abacta Bailey

Carex miliacea Muhl. ex Willd. $=$ Carex prasina Wahl.

Carex mirabilis Dewey $=$ Carex normalis Mackenzie

C. molesta Mackenzie, Troublesome Sedge

C. muhlenbergii Schkuhr ex Willd., Muhlenberg's Sedge

var. muhlenbergii

Carex muricata of some authors, not L. see Carex spicata Huds.

Carex muricata L. (of Zenkert, 1934) $=$ Carex echinata Murr.

Carex muricata var. angustata Carey $=$ Carex echinata Murr.

Carex muricata L. var. cephalantha (Bailey) Wieg. $\&$ Eames $=$ Carex echinata Murr.

Carex muricata var. sterilis (Willd.) Gleason, not Carey $=$ Carex sterilis Willd.

Carex nigromarginata var. muhlenbergii (A. Gray) Gleason $=$ Carex albicans Willd. ex Spreng. var. albicans

Carex nigromarginata var. minor (Boott) Gleason $=$ Carex crinita Lam. var. crinita

C. normalis Mackenzie, Larger Straw-sedge $=$ C. mirabilis Dewey

C. novae-angliae Schwein., New England Sedge

Carex oederi Michx. according to House, 1924, p. 195 = Carex cryptolepis Mackenzie

Carex oederi var. pumila (Cos. \& Germ.) Fern. = Carex viridula Michx.

C. oligocarpa Schkuhr ex Willd., Few-fruited Sedge

C. oligosperma Michx., Few-seeded Sedge

C. pallescens L., Pale Sedge = C. pallescens var. neogea Fern. 
Carex pallescens var. neogea Fern. $=$ Carex pallescens L.

C. pauciflora Lightf., Few-flowered Sedge

C. paupercula Michx., Bog-sedge

var. irrigua (Wahl.) Fern.

= C. magellanica ssp. irrigua (Wahl.) Hultén

In Zenkert (1934), C. paupercula is a synonym of C. magellanica of Macoun's Cat. of Can. Pl. (p. 110).

var. pallens Fern.

C. pedunculata Muhl. ex Willd., Long-stalked Sedge

Carex pellita Muhl. $=$ Carex lanuginose Michx.

C. pensylvanica Lam., Pennsylvania-sedge

C. plantaginea Lam., Plantain-leaved Sedge

C. platyphylla Carey, Broad-leaved Sedge

Carex polygama Schk. $=$ Carex buxbaumii Wahl.

Carex polytrichoides Muhl. ex Willd. = Carex leptalea Wahl.

C. prairea Dewey ex Wood, Prairie-sedge

C. prasina Wahl., Drooping Sedge

$=$ C. miliacea Muhl. ex Willd.

C. projecta Mackenzie, Necklace Sedge

C. pseudocyperus L., Cyperus-like Sedge

Carex pubescens Muhl. $=$ Carex hirtifolia Mackenzie

C. radiata (Wahl.) Small (not Dewey), Sedge

= C. flaccidula Steudel, sec. Macdonald 1990

$=$ C. rosea of some authors, not Willd.

$=$ C. rosea auct. Ontario reports in large part (Morton \& Venn 1990)

Carex retrocurva Dewey in Wood $=$ Carex laxiculmis Schwein.

C. retroflexa Muhl. ex Willd., Reflexed Sedge

C. retrorsa Schwein., Retrorse Sedge

Carex riparia (of authors) $=$ Carex lacustris Willd.

Carex riparia var. lacustris (Willd.) Kuekenth = Carex lacustris Willd.

C. rosea Schkuhr ex Willd., Rose-sedge

= C. convoluta Mackenzie, "see note under C. radiata"

C. rostrata Stokes ex With., Beaked Sedge var. utriculata (Boott) Bailey $=$ Carex utriculata Boott in Hook.

Carex rugosperma Mackenzie $=$ Carex tonsa

(Fern.) Bickn. var. rugosperma

(Mackenzie) Crins

C. sartwellii Dewey, Sartwell's Sedge

= C. disticha (of Day, 1882)
C. scabrata Schwein., Rough Sedge

C. schweinitzii Dewey ex Schwein., Schweinitz's Sedge

C. scoparia Schkuhr ex Willd., Pointed Broom Sedge

var. scoparia

C. seorsa Howe ex Gord. \& Howe, Sedge $=\mathrm{C}$. rosaeoides Howe ex Gord. \& Howe

Carex setacea Dewey $=$ Carex vulpinoidea Michx.

Carex siccata Dewey (sec. NYFA July 2005) = Carex argyrantha Tuckerm.

Carex shriveri Britt. $=$ Carex granularis Muhl. ex Willd. var. haleana (Olney) Porter

C. sparganioides Muhl. ex Willd., Bur-reed Sedge

Carex sparganioides var. cephaloidea (Dewey) Carey $=$ Carex cephaloidea (Dewey) Dewey

*C. spicata Huds., Spiked Sedge $=$ C. muricata of some authors, not L.

C. sprengelii Dewey ex Sprengel

C. squarrosa L., Squarrose Sedge

Carex stellulata Good. $=$ Carex sterilis Willd.

Carex stellulata var. cephalantha "of Gray's Man., ed 7" cited by Zenkert $(1934)=$ Carex echinata Murr.

C. sterilis Willd., Barren Prickly Sedge

$=$ C. elachycarpa Fern.

$=$ C. stellulata Good.

= C. muricata var. sterilis (Willd.) Gleason, not Carey

Carex steudelii Kunth = Carex jamesii Schw .

C. stipata Muhl. ex Willd., Awl-fruited Sedge

[C. straminea Willd. ex Schkuhr, Straw Sedge. The report of this species by Zenkert and Zander (1975) is based on a published report for Chautauqua Co. by Burgess (1877): Burgess' specimen cannot be located. Specimens of this species, which is rare in New York State, concentrate in the New York City area (House 1924, NYFA 1990). Morton and Venn (1990) state that there are no confirmed reports of this species for Ontario.]

Carex straminea of some old NY reports, not Schkuhr ex Willd. = Carex festucacea Schkuhr ex Willd.

Carex straminea Willd. var. festucacea Boott $=$ Carex festucacea Schkuhr ex Willd.

C. stricta Lam., Tussock-sedge 
$=\mathrm{C}$. angustata Boott, according to Gray's

Manual, ed 5, 1867

$=$ C. stricta var. strictior (Dewey) Carey

$=$ C. strictior Dewey ex Wood

= C. strictior Dewey ex Wood var. angustata

(Boott) Bailey

Carex stricta var. elongata (Bock) Gleason $=$ Carex emoryi Dewey ex Torrey

Carex substricta (Kuekenth) Mackenzie ex Rydb. = Carex aquatilis Wahl.

C. swanii (Fern.) Mackenzie, Swan's Sedge

Carex tenella Schk. $=$ Carex disperma Dewey

C. tenera Dewey, Slender Straw-sedge

= C. tenera var. echioides (Fern.) Wieg.

Carex tentaculata Muhl. ex Willd. $=$ Carex lurida Wahl.

C. tenuiflora Wahl., Sparse-flowered Sedge

Carex teretiuscula var. major Koch $=$ Carex diandra Schrank

C. tetanica Schkuhr ex Willd., Rigid Sedge

Carex tetanica var. woodii (Dewey) Wood $=$ Carex woodii Dewey

C. tonsa (Fern.) Bickn. var. rugosperma (Mackenzie) Crins, Umbelliform Sedge

$=\mathrm{C}$. rugosa Mackenzie

$=$ C. umbellata Schkuhr ex Willd. in part.

C. torta Boott ex Tuckerm., Twisted Sedge

C. tribuloides Wahl., Blunt Broom-sedge

Carex triceps (of authors) $=$ Carex hirsutella Mackz.

C. trichocarpa Schkuhr ex Willd. (not Muhl.), Hairy-fruited Sedge

$=$ C. trichocarpa var. turbinata Dewey

C. trisperma Dewey, Three-seeded Sedge

var. billingsii Knight, Billing's Sedge

var. trisperma

C. tuckermanii Dewey, Tuckerman Sedge

$=$ C. tuckermanii var. niagarensis C.P. Smith

Carex umbellata Schkuhr ex Willd. in part = Carex tonsa (Fern.) Bickn. var. rugosperma (Mackenzie) Crins

Carex utriculata Boott in Hook. $=$ Carex rostrata

Stokes ex With. var. utriculata (Boott)

Bailey

C. vaginata Tausch, Sheathed Sedge

Carex varia of old NY reports, not Muhl. ex Willd. $=$ Carex albicans Willd. ex Spreng. var. albicans

C. vesicaria L., Bladdery Sedge
C. virescens Muhl. ex Willd., Ribbed Sedge

C. viridula Michx., Little Green Sedge

$=$ C . chlorophila Mackenzie

$=$ C. irregularis Schwein.

= C. oederi var. pumila (Cos. \& Germ.) Fern.

C. vulpinoidea Michx., Foxtail Sedge, Fox-sedge

$=$ C. setacea Dewey

C. willdenovii Schkuhr ex Willd., Willdenow's Sedge

C. woodii Dewey, Wood's Sedge

$=$ C. tetanica var. woodii (Dewey) Wood

CARPINUS L. (Betulaceae)

Carpinus americana Michx. $=$ Carpinus caroliniana Walt.

C. caroliniana Walt., American Hornbeam, Blue Beech, Ironwood, Musclewood

ssp. virginiana (Marsh.) Furlow

= C. americana Michx.

$=\mathrm{C}$. betulus virginiana Marsh.

$=$ C. caroliniana var. virginiana (Marsh.) Fern.

Carpinus virginiana Mill. $=$ Ostrya virginiana (Mill.) K. Koch

CARUM L. (Apiaceae)

*C. carvi L., Caraway, Anise

CARYA Nutt. (Juglandaceae)

Carya alba (L.) K. Koch = Carya tomentosa $($ Lam. ex Poir.) Nutt.

Carya alba Nutt. = Carya ovata (Mill.) K. Koch

Carya amara (Michx. f.) Nutt. = Carya cordiformis

(Wang.) K. Koch

C. cordiformis (Wang.) K. Koch, Bitternut

Hickory, Swamp-hickory

= C. amara (Michx. f.) Nutt.

$=$ Hicoria cordiformis (Wang.) Britt. ex Britt. $\&$ Schafer

$=$ C. $\times$ laneyi of NY report, not Sarg.

C. glabra (Mill.) Sweet, Pignut Hickory

= C. glabra var. megacarpa (Sarg.) Sarg.

$=$ C. porcina (Michx. f.) Nutt.

$=$ Hicoria glabra (Mill.) Britt.

C. laciniosa (Michx.f.) G. Don, Big Shell-bark, Kingnut

= C. sulcata Nutt. (sec. House 1924)

Carya $\times$ laneyi of NY report, not Sarg. $=$ Carya cordiformis (Wang.) Koch 
Carya microcarpa Nutt. = Carya ovalis (Wang.) Sarg. according to Fernald: Gray's Manual 1950 p. 530

C. ovalis (Wang.) Sarg., Small-fruited Hickory

$=$ Carya microcarpa Nutt. according to

Fernald: Gray's Manual 1950 p. 530

$=$ Hicoria ovalis (Wang.) Ashe

C. ovata (Mill.) K. Koch, Shag-bark Hickory

= Carya alba Nutt. (sec. House 1924)

= Hicoria alba of some American authors, not

(L.) Nutt. ex Ell.

$=$ H. ovata (Mill.) Britt.

$=$ H. ovata (Mill.) Britt. var. fraxinifolia

(Sarg.) Ashe

$=$ H. ovata (Mill.) Britt. var. nuttallii (Sarg.)

Ashe

Carya porcina (Michx. f.) Nutt. = Carya glabra

(Mill.) Sweet

Carya sulcata Nutt. $=$ Carya laciniosa (Michx.f.) G. Don

C. tomentosa (Poir. ex Lam.) Nutt., Mockernut Hickory, White-Heart Hickory

$=$ C. alba (L.) Nutt. ex Ell. in part

= Hicoria tomentosa (Poir. ex Lam.) Raf.

Cassandra calyculata (L.) D. Don $=$ Chamaedaphne calyculata (L.) Moench

Cassia chamaecrista L. = Chamaecrista fasciculata (Michx.) Greene

Cassia diffusa of NY reports, not DC. $=$ Chamaecrista fasciculata (Michx.) Greene

Cassia fasciculata Michx. $=$ Chamaecrista fasciculata (Michx.) Greene

Cassia hebecarpa Fern. $=$ Senna hebecarpa (Fern.) Irwin \& Barneby

Cassia marylandica of NY reports, not L. $=$ Senna hebecarpa (Fern.) Irwin \& Barneby

Castalia odorata (Dryand ex Ait.) Woodr. \& Wood. $=$ Nymphaea odorata Dryand. ex Ait. ssp. odorata

Castalia lekophylla Small = Nymphaea odorata Dryand. ex Ait. ssp. odorata

Castalia tuberosa (Paine) Greene $=$ Nymphaea odorata Dryand. ex Ait. ssp. tuberosa (Paine) Wiersma \& Hellquist

CASTANEA Hill (Fagaceae)

Castanea americana Raf. $=$ Castanea dentata (Marsh.) Borkh.

C. dentata (Marsh.) Borkh., American Chestnut
$=$ C. americana Raf.

= C. vesca var. americana Michx.

Castanea vesca var. americana Michx. = Castanea dendata (Marsh.) Borkh.

CASTILLEJA Mutis ex L.f. (Scrophulariaceae)

C. coccinea (L.) Spreng., Painted Cup, Indianpaintbrush, Scarlet Paintbrush

CATALPA Scop. (Bignoniaceae)

C. speciosa (Warder ex Barney) Engelm.: Catalpa, Indian-bean, Catawba, Cigar-tree

CAULOPHYLLUM Michx. (Berberidaceae)

C. thalictroides (L.) Michx., Blue Cohosh, Papoose-root

CEANOTHUS L. (Rhamnaceae)

C. americanus L., New Jersey Tea, Redroot

CELASTRUS L. (Celastraceae)

C. orbiculata Thunb., Oriental Bittersweet

$=\mathrm{C}$. orbiculatus of authors

C. scandens L., Climbing Bittersweet, Wax-work, Staffvine, Climbing or False Bittersweet

CELTIS L. (Ulmaceae)

C. occidentalis L., Hackberry

$=$ C. canina Raf.

= C. crassifolia Lam.

$=$ C. occidentalis var. canina (Raf.) Sarg.

$=$ C. occidentalis var. pumila (Pursh) Gray

$=$ C. pumila Pursh

$=$ C. tenuifolia of authors, not Nutt.

CENCHRUS L. (Poaceae)

C. longispinus (Hack.) Fern., Sandbur

$=\mathrm{C}$. pauciflorus Benth.

CENTAUREA L. (Asteraceae)

Centaurea biebersteinii DC. $=$ Centaurea maculosa Lam.

*C. cyanus L., Bachelor's Button, Bluebottle, Corn-flower Bluet

Centaurea dubia of local authors, not Suter $=$ Centaurea nigrescens Willd.

Centaurea dubia ssp. vochinensis (Bernh. ex Reichenb.) Hayek $=$ Centaurea nigrescens Willd. 
*C. jacea L., Brown Knapweed

*C. maculosa Lam., Spotted Knapweed $=\mathrm{C}$. biebersteinii DC.

*C. nigra L., Black Knapweed, Spanish-buttons, Hardheads

$=\mathrm{C}$. nigra var. radiata $\mathrm{DC}$.

$=$ C. jacea var. nigra (L.) Briq.

Centaurea nigra var. radiata $\mathrm{DC} .=$ Centaurea nigra L.

*C. nigrescens Willd., Knapweed

= C. dubia Suter [Voss 1996, p. 513.]

$=$ C. dubia ssp. vochinensis (Bernh. ex

Reichenb.) Hayek

$=\mathrm{C}$. vochinensis Bernh. ex Reichenb.

*C. solstitialis L., Barnaby's Thistle, Yellow Starthistle, Barnaby's Thistle

Centaurea vochinensis Bernh. ex Reichenb. $=$ Centaurea nigrescens Willd.

CENTAURIUM Hill (Gentianaceae)

*C. erythraea Rafn. (not Raf.), European

Centaury

$=$ C. centaurium $(\mathrm{L}$.) Wight

$=$ C. umbellatum Gilib.

$=$ Erythraea centaurium Pers.

CEPHALANTHUS L. (Rubiaceae)

C. occidentalis L., Buttonbush

CERASTIUM L. (Caryophyllaceae)

Cerastium aquaticum L. = Myosoton aquaticum (L.) Moench

Cerastium apetalum Dumort. $=$ Cerastium glomeratum Thuill.

*C. arvense L., Field-chickweed, Meadowchickweed

= C. arvense ssp. strictum (L.) Ugbor.

$=\mathrm{C}$. arvense var. oblongifolium (Torr.)

Hollick \& Britt.

$=$ C. arvense var. villosum (Muhl.) Hollick \&

Britt.

$=$ C. campestre Greene

$=$ C. oblongifolium Torr.

$=$ C. occidentale Greene

= C. pennsylvanicum Hornem. ex DC.

$=\mathrm{C}$. pubescens Goldie

$=\mathrm{C}$. tenuifolium Pursh

$=\mathrm{C}$. velutinum Raf.

= C. villosum Muhl. ex Darl.
Cerastium campestre Greene $=$ Cerastium arvense

L.

Cerastium caespitosum Gilib. $=$ Cerastium fontanum Baumg. emend. Jalas

*C. fontanum Baumg. emend. Jalas, Common Mouse-ear Chickweed

= C. caespitosum Gilib.

$=\mathrm{C}$. fontanum ssp. triviale of NY authors, not (Link) Jalas

$=$ C. holostoides Fries

= C. glomeratum of some NY reports not Thuill.

$=\mathrm{C}$. vulgatum of NY authors, not L.

*C. glomeratum Thuill., Mouse-ear Chickweed

$=$ C. apetalum Dumort.

= C. glomeratum var. apetalum (Dumort.) Fenzl.

$=\mathrm{C}$. viscosum of authors, not $\mathrm{L}$.

Cerastium glomeratum of some NY reports not Thuill.$=$ Cerastium fontanum Baumg. emend. Jalas

Cerastium glutinosum Nutt. $=$ Cerastium nutans Raf.

Cerastium holostoides Fries $=$ Cerastium fontanum Baumg. emend. Jalas

Cerastium longipedunculatum Muhl., a nomen nudum $=$ Cerastium nutans Raf.

C. nutans Raf., Nodding Chickweed

$=$ C. glutinosum Nutt.

$=\mathrm{C}$. longipedunculatum Muhl., a nomen nudum

$=$ C. tenellum Fenzl

Cerastium oblongifolium Torr. $=$ Cerastium arvense L.

Cerastium occidentale Greene $=$ Cerastium arvense L.

Cerastium pennsylvanicum Hornem. ex DC. $=$ Cerastium arvense $\mathrm{L}$.

Cerastium pubescens Goldie $=$ Cerastium arvense L.

*C. semidecandrum L., Small Mouse-ear Chickweed, Spring Mouse-ear $=$ C. vulgatum var. semidecandrum (L.) Gray

Cerastium tenellum Fenzl $=$ Cerastium nutans Raf.

Cerastium tenuifolium Pursh $=$ Cerastium arvense L.

Cerastium velutinum Raf. $=$ Cerastium arvense $\mathrm{L}$.

Cerastium villosum Muhl. ex Darl. = Cerastium arvense L. 
Cerastium viscosum of authors, not L. $=$ Cerastium glomeratum Thuill.

Cerastium vulgatum of NY authors, not L. $=$ Cerastium fontanum Baumg. emend. Jalas

Cerastium vulgatum var. semidecandrum (L.) Gray $=$ Cerastium semidecandrum L.

Cerasus pennsylvanica (L.f.) Loisel. $=$ Prunus pensylvanica L.f.

Cerasus vulgaris Mill. $=$ Prunus cerasus L.

CERATOPHYLLUM L. (Ceratophyllaceae)

C. demersum L., Hornwort, Coontail

C. echinatum Gray, Spiny Hornwort, Coontail, Hornwort

$=\mathrm{C}$. muricatum of some authors, not Cham.

Ceratophyllum muricatum of some authors, not Cham. $=$ Ceratophyllum echinatum Gray

Cercis canadensis L., Redbud (excluded by Zander \& Pierce 1979).

CHAENOMELES Lindl. (Rosaceae)

Chaenomeles lagenaria (Loisel.) Koidzumi $=$ Chaenomeles speciosa (Sweet) Nakai

*C. speciosa (Sweet) Nakai, Japanese Quince $=$ Cydonia japonica of some authors, not (Thunb.) Pers.

$=$ Cydonia lagenaria (Loisel.) Koidzumi

= Chaenomeles lagenaria (Loisel.) Koidzumi

CHAENORRHINUM (DC.) Reichenb.

(Scrophulariaceae)

*C. minus (L.) Lange, Dwarf Snapdragon $=$ Linaria minor (L.) Desf.

CHAEROPHYLLUM L. (Apiaceae)

C. procumbens (L.) Crantz., Spreading Chervil

Chaerophyllum sylvestre L. = Anthriscus sylvestris (L.) Hoffm.

CHAMAECRISTA (L.) Moench (Fabaceae)

C. fasciculata (Michx.) Greene, Partridge-pea, Locust-weed

$=$ Cassia chamaecrista $\mathrm{L}$.

$=$ C. fasciculata Michx.

$=\mathrm{C}$. diffusa of NY reports, not DC.

$=$ Chamaecrista fascicularis Greene

CHAMAEDAPHNE Moench (Ericaceae)
C. calyculata (L.) Moench, Leather-leaf, Cassandra

$=$ Andromeda calyculata L.

= Cassandra calyculata (L.) D. Don

$=$ Chamaedaphne calyculata var. angustifolia (Ait.) Rehd.

CHAMAELIRIUM Willd. (Liliaceae)

C. luteum (L.) Gray, Blazing-star, Fairy-wand, Devil's-bit

CHAMAESYCE S. Gray (Euphorbiaceae)

Chamaesyce humistrata of NY reports, not

(Engelm.) Small $=$ Chamaesyce maculata

(L.) Small

C. maculata (L.) Small, Spotted Spurge

$=\mathrm{C}$. humistrata of NY reports, not (Engelm.) Small

$=$ Euphorbia maculata L.

$=$ E. humistrata of NY reports, not Engelm.

$=$ E. supina Raf.

C. nutans (Lag.) Small, Upright Spotted Spurge, Eyebane

$=$ Euphorbia maculata of authors in part not (L.) Small

$=$ E. nutans Lag.

$=$ E. preslii Guss.

$=$ E. hypericifolia of authors and Ontario reports

C. polygonifolia (L.) Small, Seaside Spurge $=$ Euphorbia polygonifolia L.

C. vermiculata (Raf.) House, Hairy Spurge, Worm-seeded Spurge

$=$ Euphorbia hirsuta (Torr.) Weig.

$=$ E. vermiculata Raf.

Chamemelum arvense (L.) Schreb. = Anthemis arvensis L.

Chamerion angustifolium (L.) Scop. ssp.circumvagum (Mosquin) Moldenke $=$ Epilobium angustifolium L.

Chamomilla maritima of NY reports, not (L.) Rydb. = Matricaria perforata Merat

Chamomila inodora (L.) Rydb. = Matricaria perforata Merat

Chamomila suaveolens (Pursh) Rydb. = Matricaria discoidea DC.

Cheirinia cheiranthoides $($ L. $)$ Link $=$ Erysimum cheiranthoides L. 
CHELIDONIUM L. (Papaveraceae)

*C. majus L., Celandine, Greater Celandine

CHELONE L. (Scrophulariaceae)

C. glabra L., Turtlehead

= C. glabra var. dilatata Fern. \& Wieg.

$=$ C. glabra var. elatior Raf.

CHENOPODIUM L. (Chenopodiaceae)

*C. album L., Lamb's Quarters, Goose-foot, Pigweed

*var. album

= C. album var. lanceolatum (Muhl.) Coss. \& Germ.

= C. lanceolatum Muhl.

$=\mathrm{C}$. viride $\mathrm{L}$. in part

var. missouriense (Aellen) Bassett \& Crompton, Missouri Goose-foot

$=$ C. missouriense Aellen

= C. paganum of authors, not Reich.

*C. ambrosioides L., Mexican Tea, Wormseed

$=$ C. ambrosioides var. anthelminticum (L.) Gray

$=\mathrm{C}$. ambrosioides var. chilense (Schrad.)

Speg.

$=\mathrm{C}$. anthelminicum L.

Chenopodium anthelminicum L. $=$ Chenopodium ambrosioides L.

*C. bonus-henricus L., Good-King-Henry

= Blitum bonus-henricus Reichenb.

*C. botrys L., Jerusalem Oak, Feather-geranium

C. capitatum (L.) Aschers., Strawberry Blite, Indian Paint

$=$ Blitum capitatum L.

Chenopodium chenopodioides (L.) Aellen $=$ Chenopodium rubrum $\mathrm{L}$.

Chenopodium dessicatum of authors, not A. Nels. $=$ Chenopodium pratericola Rydb.

Chenopodium dessicatum var. leptophylloides

(Murr) Wahl. $=$ Chenopodium pratericola Rydb.

Chenopodium foggii Wahl. $=$ Chenopodium pratericola Rydb.

Chenopodium gigantospermum Aellen = Chenopodium simplex (Torr.) Raf.

*C. glaucum L., Oak-leaved Goosefoot = C. glaucum ssp. salinum (Standl.) Aellen = C. salinum Standl.
Chenopodium humile Hook. $=$ Chenopodium rubrum $\mathrm{L}$.

Chenopodium hybridum of some authors, not L. = Chenopodium simplex (Torr.) Raf.

Chenopodium hybridum var. gigantospermum (Aellen) Rouleau $=$ Chenopodium simplex (Torr.) Raf.

Chenopodium hybridum var. simplex Torr. $=$ Chenopodium simplex (Torr.) Raf.

Chenopodium leptophyllum Nutt. ex Moq. in DC., not Wats. $=$ Chenopodium pratericola Rydb.

*C. murale L., Sowbane, Nettle-leaf Goosefoot

*C. polyspermum L., Many-seeded Goosefoot

*C. pratericola Rydb., Narrow-leaved Goosefoot $=$ C. dessicatum of authors, not A. Nels.

= C. dessicatum var. leptophylloides (Murr) Wahl.

$=\mathrm{C}$. foggii Wahl.

= C. leptophyllum Nutt. ex Moq. in DC.

$=$ C. pratericola var. leptophylloides (Murr) Aellen \& Just

*C. rubrum L., Red Pigweed, Red Goosefoot, Coat-blite

$=$ Blitum maritimum Nutt.

$=$ Chenopodium chenopodioides $(\mathrm{L}$.$) Aellen$

$=\mathrm{C}$. humile Hook.

$=$ C. rubrum var. humile (Hook.) S. Wats.

Chenopodium salinum Standl. $=$ Chenopodium glaucum L.

C. simplex (Torr.) Raf., Maple-leaved Goosefoot $=\mathrm{C}$. gigantospermum Aellen

$=\mathrm{C}$. hybridum of some authors, not L.

$=\mathrm{C}$. hybridum var. gigantospermum (Aellen) Rouleau

$=$ C. hybridum var. simplex Torr.

C. strictum Roth

var. glaucophyllum (Aellen) Wahl, Goosefoot

*C. urbicum L., City-goosefoot

CHIMAPHILA Pursh (Ericaceae)

C. maculata (L.) Pursh, Spotted Wintergreen

C. umbellata (L.) Bart., Wintergreen, Prince's Pine, Pipsissewa

ssp. cisatlantica (Blake) Hultén

$=$ C. umbellata var. cisatlantica Blake

Chiogenes hispidula (L.) Torr. \& A. Gray = Gaultheria hispidula (L.) Muhl. ex Bigel. 
Chrysanthemum balsamita (L.) Baill. = Balsamita major Desf.

Chrysanthemum balsamita var. tanacetoides Boiss $=$ Balsamita major Desf.

Chrysanthemum leucanthemum L. = Leucanthemum vulgare Lam.

Chrysanthemum leucanthemum var. pinnatifidum Lecoq.\& Lamotte $=$ Leucanthemum vulgare Lam.

Chrysanthemum parthenium (L.) Bernh. = Tanacetum vulgare $\mathrm{L}$.

CHIONANTHUS (Oleaceae)

*C. virginicus L. Old-man's Beard, Fringe tree

CHRYSOSPLENIUM L. (Saxifragaceae)

C. americanum Schwein. ex Hook., Golden Saxifrage, Water-mat, Water-carpet $=$ C. oppositifolium Walt.

Chrysosplenium oppositifolium Walt. $=$ Chrysosplenium americanum Schwein. ex Hook.

CICHORIUM L. (Asteraceae)

*C. intybus L., Chicory, Blue Sailors, Tattered Sailors, Cornflower, Succory, Witloof

CICUTA L. (Apiaceae)

C. bulbifera L., Bulb-bearing Water-hemlock

C. maculata L., Water-hemlock, Spotted

Cowbane, Musquash-root, Beaver-poison, Poison Hemlock

CIMICIFUGA L. (Ranunculaceae)

C. racemosa (L.) Nutt., Black Snakeroot, Black

Cohosh

= C. americana of NY report, not Michx.

CINNA L. (Poaceae)

C. arundinacea L., Woodreed, Stout Woodreed, Wood Reedgrass

C. latifolia (Trev. ex Goepp.) Griseb., Drooping Woodreed

CIRCAEA L. (Onagraceae)

C. alpina L., Smaller Enchanter's-Nightshade

Circaea alpina $\times$ lutetiana $=\mathrm{C}$. intermedia

Ehrh., Enchanter's Nightshade

$=\mathrm{C}$. canadensis Hill, not L.
= C. intermedia of Gray's Manual, ed. 7, according to Zenkert, 1934.

Circaea canadensis Hill, not L. $=$ Circaea alpina $\times$ lutetiana $=\mathrm{C}$. intermedia Ehrh.

Circaea canadensis L. = Circaea lutetiana L. ssp. canadensis (L.) Aschers. \& Magnus

Circaea latifolia Hill = Circaea lutetiana L. ssp. canadensis (L.) Aschers. \& Magnus

C. lutetiana L., Canadian Enchanter's-nightshade ssp. canadensis (L.) Aschers. \& Magnus

$=\mathrm{C}$. canadensis $\mathrm{L}$.

= C. quadrisulcata (Maxim.) Franch. \& Sav.

= C. quadrisulcata var. canadensis (L.) Hara

$=\mathrm{C}$. latifolia of authors, not Hill

Circaea quadrisulcata (Maxim.) Franch. \& Sav. = Circaea lutetiana L. ssp. canadensis (L.) Aschers. \& Magnus

Circaea quadrisulcata var. canadensis (L.) Hara = Circaea lutetiana L. ssp. canadensis (L.) Aschers. \& Magnus

CIRSIUM Mill. (Asteraceae)

C. altissimum (L.) Spreng., Tall Thistle

*C. arvense (L.) Scop., Canada Thistle

$=\mathrm{C}$. arvense var. integrifolium Wimm. \& Graebn.

= C. arvense var. vestitum Wimm. \& Graebn.

C. discolor (Muhl. ex Willd.) Spreng., Field-thistle

Cirsium lanceolatum of NY reports, not (L.) Hill = Cirsium vulgare (Savi) Tenore

C. muticum Michx., Swamp-thistle

$=\mathrm{C}$. muticum var. subpinnatifidum (Britt.) Fern.

Cirsium odoratum (Muhl. ex Bart.) Petrak, not Britt. ex Britt. \& Brown = Cirsium pumilum (Nutt.) Spreng.

C. pumilum (Nutt.) Spreng., Pasture-thistle, Small Bull-Thistle

$=$ C. odoratum (Muhl. ex Bart.) Petrak, not Britt. ex Britt. \& Brown

*C. vulgare (Savi) Tenore, Bull-thistle

$=$ C. lanceolatum of NY reports, not (L.) Hill

Cistus canadensis L.

$=$ Helianthemum canadensis (L.) Michx.

CITRULLUS Schrad. ex Eckl. \& Zeyh.

C. colocynthis (L.) Shrad. ex Eckl. \& Zeyh. Watermelon

$=$ Anguria citrullus (L.) Mill 
= C. vulgaris Schrad.

CLADIUM R. Br. (Cyperaceae)

C. mariscoides (Muhl.) Torr., Twig-rush, Bogrush

$=$ Mariscus mariscoides (Muhl.) Kuntze

CLAYTONIA L. (Portulacaceae)

C. caroliniana Michx., Broad-leaved Spring Beauty

$=$ C. latifolia Sheldon

Claytonia latifolia Sheldon $=$ Claytonia caroliniana Michx.

Claytonia multicaulis var. robusta Somes $=$ Claytonia virginica L.

Claytonia robusta $\mathrm{Rydb} .=$ Claytonia virginica $\mathrm{L}$. Claytonia simsii Sweet $=$ Claytonia virginica $\mathrm{L}$.

C. virginica L., Narrow-leaved Spring Beauty, Mayflower

$=$ C. media (DC.) Link

$=$ C. multicaulis var. robusta Somes

$=$ C. robusta Rydb.

= C. simsii Sweet

$=\mathrm{C}$. virginica var. acutiflora DC.

$=$ C. virginica var. simsii (Sweet) R. Davis

CLEMATIS L. (Ranunculaceae)

Clematis canadensis Mill. $=$ Clematis virginiana $L$.

Clematis dioscoreifolia Levl. \& Van. = Clematis terniflora DC.

Clematis fragrans Salisb. $=$ Clematis virginiana $L$.

Clematis maximowicziana Franch. \& Sav. = Clematis terniflora DC.

C. occidentalis (Hornem.) DC., Purple Clematis, Purple Virgin's Bower, Mountain-clematis $=$ Atragene americana Sims.

= Clematis verticillaris DC.

Clematis paniculata Thunb. $=$ Clematis terniflora DC.

*C. terniflora DC., Yam-leaf Clematis, Yam-leaf Virgin's-bower

$=$ C. dioscoreifolia Levl. \& Van.

= C. maximowicziana Franch. \& Sav.

$=$ C. paniculata Thunb.

Clematis verticillaris DC.$=$ Clematis occidentalis (Hornem.) DC.

C. virginiana L., Virgin's Bower, Traveller's Joy

= C. canadensis Mill.

$=$ C. fragrans Salisb.
Clepeola maritima L. = Lobularia maritima

CLINOPODIUM L. (Lamiaceae)

Clinopodium acinos (L.) Kuntze $=$ Acinos arvensis (Lam.) Dandy

Clinopodium arkansanum (Nutt.) House = Calamintha arkansana (Nutt.) Shinners

*C. vulgare L. Wild Basil, , Field-basil, Basilweed, Dog-mint $=$ Satureja vulgaris (L.) Fritsch $=\mathrm{S}$. vulgaris var. neogaea Fern . $=$ Calamintha clinopodium Benth.

CLINTONIA Raf. (Liliaceae)

C. borealis (Ait.) Raf. Yellow Clintonia, Woodlily, Bluebeads, Cornlily

Clintonia multiflora Beck according to Gray's Manual ed 1867 p. $529=$ Clintonia umbellulata (Michx.) Morong

C. umbellulata (Michx.) Morong, White Clintonia, Speckled or White Woodlily

$=\mathrm{C}$. multiflora Beck according to Gray's Manual ed 1867 p. 529

$=$ C. umbellata Torr. of Gray's Manual ed 1867 p. 529

= Convallaria umbellulata Michx. according to Gray's Manual ed 1867 p. 529

Clypeola allyssoides L. $=$ Alyssum alyssoides (L.) L.

[Cnicus benedictus L., Blessed Thistle. Specimens examined from western New York State were short-stemmed (from mowing) Sonchus arvensis L. The involucre of Cnicus benedictus is relatively high (3-4 $\mathrm{cm}$ ) and nearly hidden within ovate leaves immediately below it; the involucre has several series of broad, firm, spine-tipped bracts. The lower leaves have petioles.]

COELOGLOSSUM Hartm. (Orchidaceae)

Coeloglossum bracteatum (Muhl. ex Willd.) Hartm. $=$ Coeloglossum viride (L.) Hartm.

C. viride (L.) Hartm., Long-bracted Orchid = C. bracteatum (Muhl. ex Willd.) Hartm. $=$ C. viride var. virescens (Muhl. ex Willd.) Luer $=$ Habenaria bracteata (Willd.) R. Br. $=\mathrm{H}$. viridis $(\mathrm{L}.) \mathrm{R}$. Br. ex Ait. 
$=\mathrm{H}$. viridis var. bracteata (Muhl. ex Willd.)

A. Gray

$=$ Platanthera bracteata of George Clinton's collecting journal

COLEOGETON (Reichenb.) D. Les \& R. Haynes (Potamogetonaceae)

C. filiformis (Pers.) D. Les \& R. Haynes, Filiform Pondweed

ssp. alpinus (Blytt) D. Les \& R. Haynes

$=$ Potamogeton filiformis var. alpinus (Blytt) Aschers.

$=$ P. filiformis var. borealis (Raf.) St. John

$=\mathrm{P}$. filiformis var. macounii Morong

$=$ Stuckenia filiformis (Pers.) Börner ssp. alpinus (Blytt) Haynes, Les \& Kral

ssp. occidentalis (Robbins) D. Les \& R. Haynes

$=$ Potamogeton filiformis var. occidentalis (Robbins.) Morong

$=\mathrm{P}$. interior Rydb.

$=\mathrm{P}$. vaginatus of NY reports, not Turcz.

$=$ Stuckenia filiformis (Pers.) Börner ssp. occidentalis (Robbins) Kaynes, Les \& Kral

C. pectinatum (L.) D. Les \& R. Haynes Fennelleaved Pondweed, Sago Pondweed

$=$ Potamogeton pectinatus L.

$=$ Stuckenia pectinata (L.) Börner

COLLINSIA Nutt. (Scrophulariaceae)

C. verna Nutt., Blue-eyed Mary, Innocence

COLLINSONIA L. (Lamiaceae)

C. canadensis L., Horse-balm, Richweed, Stoneroot

COLLOMIA Nutt. (Polemoniaceae)

*C. linearis Nutt., Narrow-leaved Collomia $=$ Gilia linearis (Nutt.) A. Gray

COMANDRA Nutt. (Santalaceae)

Comandra livida Richards (excluded by Zander \& Pierce 1979).

Comandra richardsiana Fern. $=$ Comandra umbellata (L.) Nutt.

C. umbellata (L.) Nutt., Bastard Toad Flax

$=$ C. richardsiana Fern.

$=$ C. umbellata var. decumbens E. Hill

$=$ Thesium umbellatum L.
Comarum palustre L. = Potentilla palustris (L.)

Scop.

Comarum palustre var. villosum Pers. $=$ Potentilla palustris (L.) Scop.

COMMELINA L. (Commelinaceae)

*C. communis L., Asiatic Day-flower, Wandering Jew

var. communis

*var. ludens (Miq.) Pennell

COMPTONIA L'Hér. (Myricaceae)

C. peregrina (L.) Coult., Sweet-fern

= Comptonia asplenifolia (L.) L'Hér.

$=$ C. peregrina var. aspleniifolia (L.) Fern.

$=$ Liquidambar peregrina $\mathrm{L}$.

$=$ Myrica aspleniifolia L.

$=$ M. peregrina (L.) Kuntze

CONIOSELINUM Hoffm. (Apiaceae)

Conioselenum pumilum Rose $=$ Conioselenum chinense (L.) BSP.

Conioselinum canadense Torr. \& A. Gray $=$ Conioselinum chinense (L.) BSP

C. chinense (L.) BSP, Hemlock-parsley

= C. canadense Torr. \& Gray

$=\mathrm{C}$. pumilum Rose

= Selinum canadense Michx.

CONIUM L. (Apiaceae)

*C. maculatum L., Poison Hemlock

CONOPHOLIS Wallr. (Orobanchaceae)

C. americana (L.) Wallr., Squaw-root, Cancerroot

$=$ Orobanche americana L .

CONRINGIA Heist. ex Adans. (Brassicaceae)

*C. orientalis (L.) Dumort., Hare's-ear Mustard, Treacle-mustard

CONSOLIDA (DC.) S. Gray (Ranunculaceae)

*C. ajacis (L.) Schur, Rocket Larkspur

$=$ C. ambigua (L.) Ball \& Heyw.

$=$ Delphinium ajacis L.

$=\mathrm{D}$. ambiguum $\mathrm{L}$.

= D. consolida of Torrey's NY Flora, not L.

Consolida ambigua (L.) Ball \& Heyw. = Consolida ajacis (L.) Schur 
*C. regalis S. Gray, Forking Larkspur, Royal Larkspur

$=$ Delphinium consolida L.

CONVALLARIA L. (Liliaceae)

*C. majalis L., Lily-of-the-Valley

Convallaria umbellulata Michx. according to

Gray's Manual ed 1867 p. $529=$ Clintonia

umbellulata (Michx.) Morong

CONVOLVULUS L. (Convolvulaceae)

*C. arvensis L., Field-bindweed

$=$ Strophocaulos arvensis (L.) Small

Convolvulus japonicus Thunb. $=$ Calystegia hederacea Wallich

Convolvulus nashii House $=$ Calystegia sepium (L.) R. Br. ssp. sepium

Convolvulus pellitus Ledeb. $=$ Calystegia hederacea Wallich

Convolvulus sepium L. = Calystegia sepium ssp. sepium (L.) R. Br.

Convolvulus spithameus L. $=$ Calystegia spithamea (L.) Pursh

Convolvulus wallichianus Spreng. $=$ Calystegia hederacea Wallich

CONYZA Less. (Asteraceae)

Conyza asteroides L. = Aster paternus Cronq.

C. canadensis (L.) Cronq., Horseweed, Hogweed, Butterweed

var. canadensis

$=$ Erigeron canadensis L.

$=$ Leptilon canadense (L.) Britt. ex Britt. \& Brown

COPTIS Salisb. (Ranunculaceae)

Coptis groenlandica (Oeder) Fern. $=$ Coptis trifolia (L.) Salisb.

C. trifolia (L.) Salisb., Goldthread

$=$ Anemone groenlandica Oed.

$=$ Coptis groenlandica (Oeder) Fern.

$=$ C. trifolia ssp. groenlandica (Oeder) Hultén

$=\mathrm{C}$. trifolia var. groenlandica (Oeder) Fassett

$=$ Heleborus trifolius L.

Coptis trifolia (L.) Salisb. var. groenlandica

(Oeder) Fassett $=$ Coptis trifolia $(\mathrm{L}$.

Salisb.

CORALLORHIZA Gagn. (Orchidaceae)
Corallorhiza corallorhiza (L.) Karst. = Corallorhiza trifida Chat.

Corallorhiza innata R. Br. in Ait. = Corallorhiza trifida Chat. (not (L.) Chat.)

Corallorhiza macraei A. Gray = Corallorhiza striata Lindl. (excluded by Zander \& Pierce 1979).)

C. maculata (Raf.) Raf., Spotted Coral-root $=$ C. multiflora Nutt.

Corallorhiza multiflora Nutt. $=$ Corllorhiza maculata (Raf.) Raf.

C. odontorhiza (Willd.) Nutt., Autumn Coral-root

[Corallorhiza striata Lindl.: excluded for the flora by Zander and Pierce (1979): probable, documented by a specimen over 20 years old for Orleans Co. (Young 1998).]

C. trifida Chat., Early Coral-root $=$ C. corallorhiza (L.) Karst.

$=$ C. trifida var. verna (Nutt.) Fern.

$=$ C. innata R. Br. in Ait.

Corallorrhiza (orth. var.) $=$ Corallorhiza Chat .

COREOPSIS L. (Asteraceae)

Coreopsis alternifolius L. $=$ Verbesina alternifolia (L.) Britt.

Coreopsis heterogyna Fern.$=$ Coreopsis lanceolata L.

*C. lanceolata L., Lance-leaved Tickseed

$=\mathrm{C}$. heterogyna Fern.

$=\mathrm{C}$. lanceolata var. villosa Michx.

*C. tinctoria Nutt., Garden Tickseed

Coreopsis trichosperm Michx. $=$ Bidens coronata (L.) Britt.

CORIANDRUM L. (Apiaceae)

*C. sativum L., Coriander

CORISPERMUM L. (Chenopodiaceae)

Corispermum americanum (Nutt.) Nutt. = Corispermum hyssopifolium L.

*C. hyssopifolium L., Bugseed

= C. americanum (Nutt.) Nutt. var. americanum

$=$ C. pallasii Steven

= C. villosum Rydberg sec. Macdonald 1990

*C. nitidum Kitaibel ex Schultes

*C. orientale Lamarck

Corispermum pallasii Steven $=$ Corispermum hyssopifolium L. (see Mosyakin 1995). 
Corispermum villosum Rydberg sec. Macdonald $1990=$ Corispermum hyssopifolium L .

CORNUS L. (Cornaceae)

Cornus alba ssp. stolonifera (Michx.) Wang. = Cornus sericea L.

C. alternifolia L.f., Alternate-leaved Dogwood, Green Osier, Pagoda Dogwood $=$ Svida alternifolia (L.f.) Small

C. amomum Mill., Silky Cornel, Kinnikinnik ssp. amomum ssp. obliqua (Raf.) Wilson Narrow-Leaf Dogwood

= C. amomum var. schuetziana (Meyer) Rickett

$=$ C. obliqua Raf.

$=$ C. purpusi Koehne

Cornus asperifolia (of authors) $=$ Cornus drummondii Meyer

Cornus baileyi Coult. \& Evans = Cornus drummondii C.A. Meyer

C. canadensis L., Bunchberry, Dwarf Cornel, Dwarf Dogwood, Crackerberry, Puddingberry

$=\mathrm{C}$. canadensis var. alpestris House

Cornus candidissima Marsh. $=$ Cornus foemina ssp. racemosa (Lam.) J. Wilson.

Cornus circinata L'Hér. = Cornus rugosa Lam.

C. drummondii C.A. Meyer, Drummond's Dogwood, Rough-Leaf Dogwood

= C. asperifolia of NY reports, not Michx.

$=$ C. baileyi Coult. \& Evans

$=$ C. priceae Small

C. florida L., Flowering Dogwood

C. foemina Mill., Panicled Dogwood, Stiff Dogwood, Gray Dogwood

ssp. racemosa (Lam.) J. Wilson, Gray Dogwood

$=$ C. femina of authors

$=\mathrm{C}$. racemosa Lam.

$=$ C. candidissima Marsh.

= C. paniculata L'Hér.

*ssp. microcarpa (Nash) J. S. Wilson

$=$ C. microcarpa Nash

Cornus microcarpa Nash $=$ Cornus foemina ssp. microcarpa (Nash) J. S. Wilson

Cornus obliqua Raf. $=$ Cornus amomum ssp. obliqua (Raf.) Wilson
Cornus paniculata L'Hér. = Cornus foemina ssp. racemosa (Lam.) J. Wilson

Cornus priceae Small $=$ Cornus drummondii Meyer

Cornus purpusii Koehne $=$ Cornus amomum ssp. obliqua (Raf.) Wilson

Cornus racemosa Lam. $=$ Cornus foemina ssp. racemosa (Lam.) J. Wilson

C. rugosa Lam., Round-leaved Dogwood $=$ C. circinata L'Hér.

C. sericea L., Red-osier Dogwood = C. alba ssp. stolonifera (Michx.) Wang

= C. sericea var. interior (Rydb.) St. John

= C. stolonifera Michx.

= C. stolonifera var. baileyi (Coult. \& Evans) Drescher

Cornus stolonifera Michx. $=$ Cornus sericea L.

Cornus stolonifera var. baileyi (Coult. \& Evans) Drescher $=$ Cornus sericea L .

CORONILLA L. (Fabaceae)

*C. varia L., Crown Vetch

CORONOPUS Zinn (Brassicaceae)

*C. didymus (L.) Sm., Wart-cress, Wart-grass, Lesser Swinecress

$=$ Carara didyma $($ L. $)$ Britt.

CORYDALIS Vent. (Fumariaceae)

C. flavula (Raf. ex Desv.) DC., Yellow Corydalis, Yellow Harlequin

$=$ Capnoides flavula (Raf. ex Desv.) Kuntze

Corydalis glauca Pursh $=$ Corydalis sempervirens (L.) Pers.

C. sempervirens (L.) Pers., Pink Corydalis, Rock Harlequin

$=$ Capnoides sempervirens (L.) Borck. ex Roemer

$=$ Corydalis glauca Pursh

Corylaceae $=$ Betulaceae

CORYLUS L. (Betulaceae)

C. americana Walt., American Hazelnut, American Filbert, Hazel

*C. avellana L., European Filbert, European Hazel

C. cornuta Marsh., Beaked Hazelnut $=\mathrm{C}$. rostrata Ait.

Corylus rostrata Ait. $=$ Corylus cornuta Marsh.

COSMOS Cav. (Asteraceae) 
*C. bipinnatus Cav., Cosmos

COTINUS Mill. (Anacardiaceae)

*C. coggygria Scop., Smoke-tree

$=$ Rhus cotinus $\mathrm{L}$.

Not recorded for the Ontario flora by Morton \&

Venn (1990), it has escaped and flourishes

at Artpark, Lewiston, New York, just

across the river from Queenston, Ontario.

Recorded for New York State by Mitchell and Tucker (1997).

CRATAEGUS L. (Rosaceae)

Crataegus acclivis Sarg. $=$ Crataegus pedicellata

Sarg.

Crataegus albicans Ashe $=$ Crataegus pedicellata

Sarg.

Crataegus arcana Beadle $=$ Crataegus pruinosa

(Wendl. f.) K. Koch

Crataegus aulica Sarg. $=$ Crataegus coccinioides

Ashe

Crataegus basilica Beadle $=$ Crataegus schuettii

Ashe

Crataegus baxteri Sarg. $=$ Crataegus intricata

Lange

C. beata Sarg., Opulent Hawthorn

$=$ C. macrocalyx Sarg.

Crataegus beata var. compta (Sarg.) Eggl. =

Crataegus compta Sarg.

Crataegus boyntonii Beadle $=$ Crataegus intricata Lange

Crataegus brachyloba Sarg. $=$ Crataegus brainerdii Sarg.

C. brainerdii Sarg., Brainerd's Thorn

$=$ C. brachyloba Sarg.

Crataegus brainerdii var. asperifolia (Sarg.) Eggl. = Crataegus scabrida Rehd.

Crataegus brainerdii var. egglestonii (Sarg.)

Robins. $=$ Crataegus scabrida Rehd .

Crataegus brainerdii var. scabrida (Sarg.) Eggl. = Crataegus scabrida Rehd.

Crataegus bushii auct. non Sarg. = Crataegus crusgalli L.

C. calpodendron (Ehrh.) Medik., Pear Thorn

$=$ C. calpodendron $\times$ punctata Eggl. according to Zenkert, following Sargent

1913 as a synonym of C. celsa Sarg.

$=$ C. celsa Sarg.

$=$ C. tomentosa Sarg. in part
Crataegus celsa Sarg. $=$ Crataegus calpodendron (Ehrh.) Medik.

C. chrysocarpa Ashe, Round-leaved Hawthorn = C. chrysocarpa var. faxonii (Sarg.) Eggl.

= C. dodgei Sarg.

$=\mathrm{C}$. rotundifolia (Ehrh.) Moench in part

Crataegus chrysocarpa var. faxonii (Sarg.) Eggl. = Crataegus chrysocarpa Ashe

Crataegus clintoniana Sarg. $=$ Crataegus suborbiculata Sarg.

Crataegus coccinea of American authors in large part, not L., and of Zenkert $1934=$ Crataegus pedicellata Sarg.

Crataegus coccinea var. ellwangeriana (Sarg.) Eggl. = Crataegus pedicellata Sarg.

C. coccinioides Ashe, Kansas Hawthorn

= C. aulica Sarg.

= C. coccinioides var. dilatata (Sarg.) Eggl. (of Zenkert 1934).

$=$ C. conspecta Sarg.

= C. dilatata Sarg.

= C. durobrivensis Sarg.

$=$ C. eggertii Britt.

Crataegus coccinioides var. dilatata (Sarg.) Eggl. = Crataegus coccinioides Ashe

Crataegus cognata Sarg. (after Zenkert 1934 p. $177)=$ Crataegus pruinosa (Wendl. f.) $\mathrm{K}$. Koch

C. compta Sarg., Hawthorn

= C. beata var. compta (Sarg.) Eggl.

$=\mathrm{C}$. formosa Sarg. (also a synonym of C. pruinosa (Wendl.f.) Koch)

$=$ C. levis Sarg.

= C. pulchra Sarg.

= C. strigosa Sarg.

Crataegus conspecta Sarg. $=$ Crataegus coccinioides Ashe

[Crataegus corusca, Hawthorn (taxonomic question, Regl. M. Niag. Oldham 1999]

C. crus-galli L. Cockspur Thorn

$=$ C . crus-galli var. prunifolia (Poir.) Torrey \& A. Gray

$=$ C . crus-galli var. pyracanthifolia Ait. (of Zenkert 1934)

= C. persimilis Sarg. (see Phipps 1988 sec. Morton \& Venn 1990)

$=$ C. prunifolia (Poir.) Pers. 
[C. crus-galli $\times$ succulenta Eggl. "This hybrid is known from Buffalo and Niagara Falls"

(Zenkert 1934 citing House 1924 p. 416)

Crataegus dayana Sarg. $=$ Crataegus pedicellata

Sarg.

Crataegus dilatata Sarg. $=$ Crataegus coccinioides

Ashe

Crataegus dissona Sarg. $=$ Crataegus pruinosa

(Wendl f.) K. Koch s.l.

Crataegus dodgei Sarg. = Crataegus chrysocarpa

Ashe

Crataegus durobrivensis Sarg. $=$ Crataegus

coccinioides Ashe

Crataegus eggertii Britt. $=$ Crataegus coccinioides

Ashe

Crataegus exclusa Sarg. $=$ Crataegus pringlei Sarg .

Crataegus ferentaria Sarg. $=$ Crataegus succulenta

Link

Crataegus filipes Ashe $=$ Crataegus jesupii Sarg.

(of NYFA 1990)

Crataegus filipes Ashe (sensu Zenkert 1934 and

Sargent 1913) $=$ Crataegus opulens Sarg.

Crataegus finitima Sarg. $=$ Crataegus succulenta

Link

C. flabellata (Bosc) Koch [not (Spach) Kirchner], Variable Thorn

Crataegus foetida Ashe $=$ Crataegus intricata

Lange

Crataegus formosa Sarg. $=$ Crataegus pruinosa

(Wendl. f.) K. Koch (also synonymized

under C. compta Sarg. by Mitchell \&

Tucker 1997).

Crataegus genialis Sarg. = Crataegus macrosperma

Ashe

Crataegus gracilis Sarg. = C. pruinosa (Wendl. f.)

K. Koch s.l.

C. holmesiana Ashe, Holmes' Hawthorn

$=$ C. tardipes (Sarg.) Sarg.

Crataegus implicata Sarg. $=$ Crataegus menandiana

Sarg.

Crataegus incisa Sarg. = Crataegus jesupii Sarg.

C. intricata Lange, Biltmore Thorn

$=$ C. baxterii Sarg.

$=$ C . boyntonii Beadle (of Zenkert 1934)

= C. foetida Ashe

C. jesupii Sarg., Hawthorn

$=\mathrm{C}$. filipes Ashe

$=$ C. numerosa Sarg.

$=\mathrm{C}$ incisa Sarg.
Crataegus leiophylla Sarg. $=$ Crataegus pruinosa

(Wendl f.) K. Koch s.l.

Crataegus leiophylla var. maineana (Sarg.) Eggl. =

Crataegus pruinosa (Wendl f.) K. Koch s.l.

Crataegus letchworthiana Sarg. $=$ Crataegus

pedicellata Sarg.

Crataegus levis Sarg. = Crataegus compta Sarg.

Crataegus lobulata Sarg. = Crataegus pringlei Sarg.

Crataegus luminosa Sarg. $=$ Crataegus schuettii

Ashe

Crataegus macrantha Lodd.$=$ Crataegus succulenta Link

Crataegus macrocalyx Sarg. $=$ Crataegus beata Sarg.

C. macrosperma Ashe, s.l., Large-fruited

Hawthorn

$=$ C. genialis Sarg.

= C. macrosperma var. acutiloba (Sarg.)

Eggl. (of Zenkert 1934).

= C. macrosperma var. pastorum (Sarg.)

Eggl.

$=\mathrm{C}$. macrosperma var. roanensis (Ashe)

Palmer

$=$ C. roanensis Ashe (of Zenkert 1934)

Crataegus macrosperma $\times$ pruinosa $=$ Crataegus

menandiana Sarg.

Crataegus macrosperma var. roanensis (Ashe)

Palmer $=$ Crataegus macrosperma Ashe

C. menandiana Sarg., Hawthorn

= C. implicata Sarg.

$=\mathrm{C}$. macrosperma $\times$ pruinosa (of Zenkert 1934)

Crataegus mollis (Torr. \& A. Gray) Scheele sensu Soper $\&$ Heimberger in our region $=$ Crataegus submollis Sarg.

*C. monogyna Jacq., English Hawthorn

$=\mathrm{C}$. oxyacantha (of authors)

Crataegus neofluvialis Ashe $=$ Crataegus succulenta Link

Crataegus notabilis Sarg. $=$ Crataegus suborbiculata Sarg.

Crataegus numerosa Sarg. $=$ Crataegus jesupii Sarg.

Crataegus oxyacantha (of authors) $=$ Crataegus monogyna Jacq.

C. opulens Sarg., Opulent Hawthorn $=$ C. filipes Ashe

C. pedicellata Sarg., Scarlet Thorn, Red Haw, Red Hawthorn 
$=$ C. acclivis Sarg.

= C. albicans Ashe (of Zenkert 1934)

$=$ C. coccinea L. American authors in large part, not L.; of Zenkert 1934

= C. coccinea L. var. ellwangeriana (Sarg.)

Eggl. (of Zenkert 1934)

$=$ C. dayana Sarg.

$=$ C. letchworthiana Sarg.

$=$ C. pedicellata var. albicans (Ashe) Palmer

$=$ C. pedicellata var. ellwangeriana (Sarg.) Eggl.

C. persimilis Sarg. = C. crus-galli L.

C. pringlei Sarg., Pringle's Hawthorn

$=$ C. exclusa Sarg.

= C. lobulata Sarg.

= C. pringlei var. exclusa (Sarg.) Eggl.

$=$ C. pringlei var. lobulata (Sarg.) Eggl.

C. pruinosa (Wendl. f.) K. Koch s.l., Waxy-fruited Thorn

$=$ C. arcana Beadle

= C. dissona Sarg.

= C. cognata Sarg. (of Zenkert 1934 p. 177)

= C. formosa Sarg.

$=$ C. gracilis Sarg.

= C. leiophylla Sarg. (of Zenkert 1934)

= C. leiophylla var. maineana (Sarg.) Eggl. (of Zenkert 1934)

= C. pruinosa var. conjucta (Sarg.) Eggl.

= C. pruinosa var. dissona (Sarg.) Eggl.

$=\mathrm{C}$. pruinosa var. latisepala (Ashe) Eggl.

$=\mathrm{C}$. rugosa Ashe

Crataegus prunifolia (Poir.) Pers. $=$ Crataegus crusgalli L.

Crataegus pulchra Sarg. $=$ Crataegus compta Sarg.

C. punctata Jacq., Dotted Hawthorn

$=$ C. punctata var. aurea Ait.

Crataegus roanensis Ashe $=$ Crataegus macrosperma Ashe

Crataegus rotundifolia (Ehrh.) Moench in part = Crataegus chrysocarpa Ashe sensu lato

Crataegus rugosa Ashe $=$ Crataegus pruinosa (Wendl f.) K. Koch s.l.

C. scabrida Rehd., Hawthorn

= C. brainerdii var. asperifolia (Sarg.) Eggl. (of Zenkert 1934)

$=$ C. brainerdii var. egglestonii (Sarg.)

Robins. (of Zenkert 1934)

= C. brainerdii var. scabrida (Sarg.) Eggl. (of Zenkert 1934)
C. schuettii Ashe, Hawthorn

= C. basilica Beadle (of House 1924 p. 421)

$=$ C. luminosa Sarg.

Crataegus strigosa Sarg. = Crataegus compta Sarg.

C. submollis Sarg., Downy Thorn

$=$ C. mollis (Torr. \& A. Gray) Scheele sensu

Soper \& Heimberger 1982 for our region

C. suborbiculata Sarg., Hawthorn

$=$ C. clintoniana Sarg.

$=$ C. notabilis Sarg.

Crataegus venustula Sarg. $=$ Crataegus succulenta Link

C. succulenta Link, Long-spined Thorn

$=$ C. ferentaria Sarg.

= C. finitima Sarg.

$=$ C. macrantha Lodd.

$=$ C. neofluvialis Ashe

$=$ C. succulenta var. macracantha (Lodd.)

Eggl.

$=$ C. succulenta var. neofluvialis (Ashe)

Palm.

= C. succulenta var. rhombifolia (Sarg.) Eggl.

= C. venustula Sarg.

Crataegus succulenta var. neofluvialis (Ashe)

Palm. $=$ Crataegus succulenta Link

Crataegus succulenta var. rhombifolia (Sarg.) Eggl.

$=$ Crataegus succulenta Link

Crataegus tardipes (Sarg.) Sarg. $=$ Crataegus

holmesiana Ashe

Crataegus tomentosa Sarg. in part $=$ Crataegus calpodendron (Ehrh.) Medik.

Crataegus venustula Sarg. $=$ Crataegus succulenta Link

CREPIS L. (Asteraceae)

*C. biennis L., Rough Hawk's-beard

*C. capillaris (L.) Wallr., Smooth Hawk's-beard

*C. tectorum L., Hawk's-beard

Crocanthemum canadense (L.) Britt. ex Britt. \&

Brown $=$ Melianthemum canadense $(\mathrm{L}$.

Michx.

Cruciferae $=$ Brassicaceae

CRYPSIS Ait. (Poaceae)

*C. schoenoides (L.) Lam., Crypsis, Heleochloa, Swamp Timothy

= Heleochloa schoenoides (L.) Host

CRYPTOGRAMMA R. Br. (Pteridaceae) 
C. stelleri (S. Gmel.) Prantl ex Engl., Slender Cliff-brake

CRYPTOTAENIA DC. (Apiaceae)

C. canadensis (L.) DC., Honewort, Wild Chervil $=$ Deringa canadensis (L.) Kuntze

Cubelium concolor (Forst.) Raf. $=$ Hybanthus concolor (Forst.) Spreng.

CUCUMIS L. (Cucurbitaceae)

*C. sativus L., Garden Cucumber

CUCURBITA L. (Cucurbitaceae)

*C. pepo L., Pumpkin, Acorn Squash, Summer Squash

= C. moschata (Dcne. ex Lam.) Dcne. ex Poir.

$=$ Pepo moschatus (Dcne. ex Lam.) Britt.

$=$ P. pepo (L.) Britt. ex Small

$=\mathrm{P}$. vulgaris $(\mathrm{L}$.$) Moench$

CUPHEA P. Browne (Lythraceae)

Cuphea petiolata (L.) Koehne $=$ Cuphea viscosissima Jacq.

C. viscosissima Jacq., Clammy Cuphea, Tarweed, Blue Waxweed

$=$ C. petiolata $($ L.) Koehne

$=$ Lythrum petiolatum $\mathrm{L}$.

$=$ Parsonsia petiolata (L.) Rusby

CUSCUTA L. (Cuscutaceae)

Cuscuta campestris Uncker $=$ Cuscuta pentagona Engelm.

C. coryli Engelm., Hazel-dodder, Strangleweed $=\mathrm{C}$. inflexa Engelm.

*C. epilinum Weihe, Flax-dodder

*C. epithymum (L.) L., Clover-dodder

C. gronovii Willd. ex Schultz, Common Dodder,

Love-vine, Stangleweed, Goldthread-vine $=\mathrm{C}$. gronovii var. latiflora Engelm.

$=$ C. gronovii var. vulgivaga Engelm.

Cuscuta inflexa Engelm. = Cuscuta coryli Engelm.

C. pentagona Engelm., Field-dodder $=$ C. campestris Uncker

C. polygonorum Engelm., Polygonum-dodder

CYCLOLOMA Moq. (Chenopodiaceae) *C. atriplicifolium (Spreng.) Coult., Winged Pigweed, Tumbleweed
$=$ Salsola atriplicifolia Spreng.

Cydonia japonica of some authors, not (Thunb.)

Pers. $=$ Chaenomeles speciosa $($ Sweet $)$

Nakai

Cydonia lagenaria (Loisel.) Koidzumi = Chaenomeles speciosa (Sweet) Nakai

CYMBALARIA Hill (Scrophulariaceae)

*C. muralis Gaertn., Mey. \& Sherb., Kenilworth Ivy, Coliseum-ivy

$=$ Linaria cymbalaria (L.) Mill.

CYNANCHUM L. (Asclepiadaceae)

*C. laeve (Michx.) Pers., Sand-vine, Honey-vine = Ampelamus albidus (Nutt.) Britt.

= Gonolobus laevis Michx.

*C. louiseae Kartesz \& Gandhi, Black Swallowwort.

$=$ C. nigrum (L.) Pers.

$=$ Vincetoxicum nigrum (L.) Moench

Cynanchum medium of authors, not R. Br. = Cynanchum rossicum (Kleop.) Borh.

Cynanchum nigrum (L.) Pers. = Cynanchum louiseae Kartesz \& Gandhi

Cynanchum nigrum in part $=$ Cynanchum rossicum (Kleop.) Borh.

*C. rossicum (Kleop.) Borh. Swallow-wort $=\mathrm{C}$. medium of authors, not R. Br.

$=\mathrm{C}$. nigrum in part

$=$ Vincetoxicum rossicum (Kleopov) Barb.

*C. vincetoxicum (L.) Pers., White Swallow-wort $=$ Vincetoxicum album (Mill.) Aschers.

$=\mathrm{V}$. hirundinaria Medik.

$=\mathrm{V}$. vincetoxicum $(\mathrm{L}$.$) Pers.$

CYNODON L. Rich. (Poaceae)

*C. dactylon (L.) Pers., Bermuda-grass

$=$ Capriola dactylon (L.) Kuntze

CYNOGLOSSUM L. (Boraginaceae)

Cynoglossum morisonii A.DC.$=$ Hackelia virginiana (L.) Johnst.

*C. officinale L., Hound's-tongue

C. virginianum L., Northern Wild Comfrey = C. virginicum L. of Gray's Manual, ed 5, 1867, p. 366

var. boreale (Fern.) Cooperrider $=\mathrm{C}$. boreale Fern. 
Cynoglossum virginicum (of Day, 1882) = Cynoglossum virginianum L. var. boreale (Fern.) Cooperrider

CYNOSURUS L. (Poaceae)

*C. cristatus L., Dog's-tail Grass, Crested Dog's Tail

CYPERUS L. (Cyperaceae)

C. bipartitus Torr., Shining Cyperus, Rivershore Galingale, Flatsedge

$=\mathrm{C}$. rivularis Kunth

$=\mathrm{C}$. diandrus var. castaneus Torr.

C. diandrus Torr., Galingale, Umbrella Flatsedge $=\mathrm{C}$. diandrus var. elongatus Britt.

$=\mathrm{C}$. fuscus of NY reports, not L.

Cyperus diandrus var. castaneus Torr. $=$ Cyperus bipartitus Torr.

Cyperus engelmannii Steud. $=$ Cyperus odoratus L.

C. erythrorhizos Muhl., Red-rooted Cyperus, Flatsedge

*C. esculentus L., Yellow Nut Grass

$=$ "C. phymatodes Muhl.of Day's Pl. of Buf." according to Zenkert, 1934 (p. 102) and House 1924 p.133.

= C. esculentus L. var. leptostachyus Boeck.

Cyperus ferax Rich. $=$ Cyperus odoratus L.

Cyperus ferruginescens Boeckl. $=$ Cyperus odoratus L.

Cyperus filiculmis of authors, not Vahl = Cyperus lupulinus (Spreng.) Marcks ssp. lupulinus

Cyperus filiculmis var. macilentus Fern. = Cyperus lupulinus (Spreng.) Marcks ssp. macilentus (Fern.) Marcks

C. flavescens L. Galingale, Yellow Cyperus

$=$ C. flavescens var. poaeformis (Pursh) Fern.

*C. fuscus L., Dark Galingale, Flatsedge

C. lupulinus (Spreng.) Marcks, Slender Galingale ssp. macilentus (Fern.) Marcks

$=\mathrm{C}$. filiculmis var. macilentus Fern.

Cyperus michauxianus Schultes of Gray's Manual ed 5, $1867=$ Cyperus speciosus Vahl. of House 1924 = Cyperus odoratus L.

C. odoratus L., Fragrant Cyperus, Fragrant Umbrella-sedge

$=$ C. . engelmannii Steud .

$=\mathrm{C}$. ferruginascens Boeckl.

$=$ C. ferax Rich
$=$ C. michauxianus Schultes of Gray's

Manual ed 5, according to House 1924.

$=\mathrm{C}$. speciosus Vahl

$=\mathrm{C}$. odoratus L. var. squarrosus (Britton)

Gill. sensu McIntosh \& Catling 1979

"C[yperus]. phymatodes Muhl. of Day's Pl. of

Buf." according to Zenkert, 1934 (p. 102)

and House $1924=$ Cyperus esculentus L.

Cyperus rivularis Kunth $=$ Cyperus bipartitus Torr.

C. schweinitzii Torr., Schweinitz's Cyperus

Cyperus speciosus Vahl = Cyperus odoratus L.

C. strigosus L., Straw-colored Cyperus, Flatsedge $=\mathrm{C}$. strigosus var. robustior Britt.

CYPRIPEDIUM L. (Orchidaceae)

C. acaule Ait., Moccasin-flower, Pink Lady's Slipper

Cypripedium calceolus var. parviflorum (Salisb.) Fern. $=$ Cypripedium parviflorum Salisb. var. parviflorum

Cypripedium calceolus var. planipetalum (Fern.) Vict. \& Rouss. = Cypripedium parviflorum Salisb. var. pubescens (Willd.) Knight

Cypripedium calceolus var. pubescens (Willd.) Correll $=$ Cypripedium parviflorum Salisb. var. pubescens (Willd.) Knight

C. candidum Muhl. ex Willd., White Lady-slipper

Cypripedium flavescens DC. $=$ Cypripedium parviflorum Salisb. var. pubescens (Willd.) Knight

C. parviflorum Salisb., Yellow Lady-slipper

$=\mathrm{C}$. calceolus of American authors, not L. var. parviflorum Small Yellow Lady-slipper

= C. calceolus var. parviflorum (Salisb.) Fern.

var. pubescens (Willd.) Knight, Large Yellow Lady-slipper

= C. calceolus var. planipetalum (Fern.) Vict. \& Rouss.

$=$ C. calceolus var. pubescens (Willd.) Correll

$=\mathrm{C}$. flavescens DC.

= C. parviflorum var. planipetalum Fern.

$=$ C. pubescens Willd.

Cypripedium pubescens Willd. = Cypripedium parviflorum Salisb. var. pubescens (Willd.) Knight

C. reginae Walt., Showy Lady-slipper $=$ C. spectabile Salisb. 
Cypripedium spectabile Salisb. $=$ Cypripedium regina Walt.

CYSTOPTERIS Bernh. (Dryopteridaceae)

C. bulbifera (L.) Bernh., Bulblet Bladder-fern

$=$ Filix bulbifera $(\mathrm{L}$.$) Underw.$

$=$ Polypodium bulbiferum L.

C. fragilis (L.) Bernh., Brittle Bladder-fern $=$ Filix fragilis $($ L.) Gilib.

$=$ Polypodium fragile $\mathrm{L}$.

Cystopteris fragilis var. mackayi Laws. =

Cystopteris tenuis (Michx.) Desv.

Cystopteris fragilis var. protrusa Weath. =

Cystopteris protrusa (Weath.) Blasd.

C. protrusa (Weath.) Blasd., Lowland Fragile

Fern

$=$ C. fragilis var. protrusa Weath.

C. tenuis (Michx.) Desv., Fragile Fern $=\mathrm{C}$. fragilis var. mackayi Laws.

Cytherea bulbosa (L.) Salisb. = Calypso bulbosa (L.) Oakes

CYTISUS L. (Fabaceae)

*C. scoparius (L.) Link, Scotch Broom

= Genista scoparia (L.) Lam.

$=$ Sarothamnus scoparius (L.) Wimmer

$=$ Spartium scoparium L.

DACTYLIS L. (Poaceae)

*D. glomerata L., Orchard-grass

$=\mathrm{D}$. glomerata varieties

DALIBARDA L. (Rosaceae)

Dalibarda fragarioides Michx. $=$ Waldsteinia

fragarioides (Michx.) Tratt.

D. repens L., Dewdrop, False Violet, Robinrunaway, Dewdrop

$=\mathrm{D}$. violaeoides Michx.

= Rubus dalibarda L.

Dalibarda violaeoides Michx. $=$ Dalibarda repens L.

DANTHONIA Lam. \& DC. (Poaceae)

D. compressa Austin, Austin's Oatgrass, Northern Oatgrass

D. spicata (L.) Beauv. ex R. \& S., Poverty-grass, Junegrass, White Oatgrass

$=$ D. $\times$ allenii Austin

= D. spicata var. longipila Scribn. \& Merr.
DAPHNE L. (Thymelaeaceae)

*D. mezereum L., Mezereum, Daphne

Dasiphora fruticosa auct. non (L.) Rydb. =

Potentilla fruticosa ssp. floribunda (Pursh)

Elkington

Dasiphora floribunda (Pursh) Kartesz $=$ Potentilla fruticosa L. ssp. floribunda (Pursh)

Elkington

Dasystephana grayi of NY reports, not (Kusn.)

Britt. $=$ Gentiana puberulenta Pringle

Datisca hirta L. = Rhus hirta (L.) Sudworth

DATURA L. (Solanaceae)

*D. stramonium L., Jimsonweed, Stramonium, Common Thorn-apple, Jamestown Weed, Purple Thorn-apple

$=$ D. tatula L.

= D. stramonium var. tatula (L.) Torr.

Datura tatula L. = Datura stramonium L.

DAUCUS L. (Apiaceae)

*D. carota L., Queen Anne's Lace, Wild Carrot

DECODON J. Gmel. (Lythraceae)

D. verticillatus (L.) Ell., Swamp Loosestrife,

Water-willow, Water-oleander

= D. verticillatus var. laevigatus Torr. \& A.

Gray

$=$ Lythrum verticllatum $\mathrm{L}$.

= Nesaea verticillata (L.) HBK

Delphinium ajacis L. of ON reports = Consolida ajacis (L.) Schur

Delphinium ambiguum L. = Consolida ajacis (L.) Schur

Delphinium consolida of Torrey's NY Flora, not L. $=$ Consolida ajacis (L.) Schur

Delphinium consolida L. = Consolida regalis $\mathrm{S}$. Gray

Dendrolycopodium dendroideum (Michx.) A. Haines $=$ Lycopodium dendroideum Michx.

Dendrolycopodium obscurum (L.) A. Haines = Lycopodium obscurum L.

DENNSTAEDTIA Bernh. (Dennstaedtiaceae)

D. punctilobula (Michx.) Moore, Hay-scented Fern

$=$ Dicksonia pilosiuscula Willd. 
$=$ Nephrodium punctilobulum Michx.

Dentaria concatenata Michx. $=$ Cardamine concatenata (Michx.) Schwein.

Dentaria diphylla Michx. $=$ Cardamine diphylla (Michx.) Wood

Dentaria diphylla $\times$ laciniata $=[$ Cardamine concatenata $\times$ diphylla $=$ C. $\times$ maxima (Nutt.) Wood]

Dentaria diphylla $\times$ maxima $=[$ Cardamine concatenata $\times$ diphylla $=$ C.$\times$ maxima (Nutt.) Wood]

Dentaria $\times$ incisa K. Schum. $=[$ Cardamine concatenata $\times$ diphylla $=$ C. $\times$ maxima (Nutt.) Wood]

Dentaria $\times$ incisifolia Eames $=[$ Cardamine concatenata $\times$ diphylla $=$ C. $\times$ maxima (Nutt.) Wood]

Dentaria laciniata Muhl. ex Willd. $=$ Cardamine concatenata (Michx.) Schwein.

Dentaria laciniata $\times$ maxima Nutt. $=[$ Cardamine concatenata $\times$ diphylla $=$ C. $\times$ maxima (Nutt.) Wood]

Dentaria maxima Nutt. $=[$ Cardamine concatenata $\times$ diphylla $=$ C. $\times$ maxima (Nutt.) Wood]

DEPARIA Hooker \& Grev. (Dryopteridaceae)

D. acrostichoides (Sw.) Kato, Silvery Spleenwort, Silvery Gladefern

$=$ Asplenium acrostichoides Sw.

$=\mathrm{A}$. thelypteroides Michx.

$=$ Athyrium acrosticoides (Sw.) Diels

= A. thelypteroides (Michx.) Desv.

$=$ Diplazium acrostichoides (Sw.) Butters

Deringa canadensis (L.) Kuntze $=$ Cryptotaenia canadensis (L.) DC.

DESCHAMPSIA Beauv. (Poaceae)

D. cespitosa (L.) Beauv., Tufted Hair-grass

= Aira caespitosa L.

D. flexuosa (L.) Trin., Wavy Hair-grass, Common Hair-grass

= Aira flexuosa $\mathrm{L}$.

DESCURAINIA Webb \& Berth. (Brassicaceae)

D. pinnata (Walt.) Britt., Tansy Mustard ssp. brachycarpa (Richardson ex Frankl.)

Detl., Northern Tansy-mustard = D. pinnata var. brachycarpa (Rich.) Fern.
$=$ Sisymbrium brachycarpon Richardson (Morton \& Venn 1990)

= S. brachycarpon Rich. ex Frankl.

(Mitchell \& Tucker 1997)

$=\mathrm{S}$. canescens as to ON reports, not of Nutt. (Morton \& Venn 1990)

[*ssp. pinnata, Southern Tansy-mustard (= Sisymbrium canescens), excluded by Zander and Pierce (1979).]

Descurainia pinnata var. brachycarpa (Richards.)

Fern.$=$ Descurainia pinnata ssp. brachycarpa (Richards. ex Frankl.) Detl.

*D. sophia (L.) Webb ex Prantl, Herb Sophia $=$ Sisymbrium sophia L.

DESMODIUM Desv. (Fabaceae)

Desmodium acuminatum DC. $=$ Desmodium glutinosum (Muhl. ex Willd.) Wood

Desmodium bracteosum (Michx.) DC. $=$ Desmodium cuspidatum (Muhl. ex Willd.) DC. ex Loud.

D. canadense (L.) DC., Canadian Tick Trefoil, Giant Tick-clover or -trefoil, Showy Ticktrefoil, Beggar-ticks

Desmodium canescens (L.) DC. (excluded by Zander \& Pierce 1979).

D. ciliare (Muhl. ex Willd.) DC, Hairy Smallflowered Tick-trefoil

$=\mathrm{D}$. obtusum of authors

D. cuspidatum (Muhl. ex Willd.) DC. ex Loud., Pointed-leaved Tick-trefoil

$=$ D. bracteosum (Michx.) DC.

$=\mathrm{D}$. grandiflorum (Walt) DC.

Desmodium dillenii of some authors, not Darl. = Desmodium glabellum (Michx.) DC.

Desmodium dillenii Darl. in part $=$ Desmodium perplexum Schub.

Desmodium dillenii Darl. $=$ Desmodium paniculatum (L.) DC.

D. glabellum (Michx.) DC., Dillen's Tick-trefoil, Tall Tick-clover or Tick-trefoil $=$ D. dillenii of some authors, not Darl. $=\mathrm{D}$. humifusum of some NY reports, not (Muhl.) Beck

D. glutinosum (Muhl. ex Willd.) Wood, Sticky Tick-trefoil = D. acuminatum DC. 
Desmodium grandiflorum (Walt) DC. = Desmodium cuspidatum (Muhl. ex Willd.) DC. ex Loud.

Desmodium humifusum of some NY reports, not (Muhl.) Beck $=$ Desmodium glabellum (Michx.) DC.

D. marilandicum (L.) DC., Smooth Small-leaved Tick-trefoil, Maryland Tick-clover

D. nudiflorum (L.) DC., Naked-flowered Ticktrefoil, Bare-stemmed Tick-trefoil

Desmodium obtusum (of authors) $=$ Desmodium ciliare (Muhl. ex Willd.) DC.

[D. obtusum (Muh. ex Willd.) DC. ( = D. rigidum (Ell.) DC.) Erie Co. report not corroborated by specimen (Young 1998).]

D. paniculatum (L.) DC., Panicled Tick-trefoil $=$ D. dillenii Darl.

D. pauciflorum (Nutt.) DC., Few-or Smallflowered Tick-trefoil

D. perplexum Schub., Tick-clover, Tick-trefoil $=$ D. dillenii Darl. in part

$=$ Meibomia dillenii (Darl.) Kuntze in part

Desmodium rigidum (Ell.) DC. $=$ Desmodium obtusum (Muh. ex Willd. DC. (excluded by Zander \& Pierce 1979).

D. rotundifolium DC., Round-leaved Tick-trefoil Dianthera americana L. = Justicia americana (L.) Vahl

DIANTHUS L. (Caryophyllaceae)

*D. armeria L., Deptford Pink

*D. barbatus L., Sweet William

Dianthus blandus (Reichenb.) Hayek = Dianthus deltoides L.

*D. deltoides L., Maiden-pink, Meadow-pink

Dianthus hoppei Portenschl. $=$ Dianthus deltoides L.

Dianthus luminitzeri Weisb. $=$ Dianthus deltoides L.

Dianthus praecox Kit. ex Willd. $=$ Dianthus deltoides $\mathrm{L}$.

DICENTRA Bernh. (Fumariaceae)

D. canadensis (Goldie) Walp., Squirrel-corn, Turkey-corn $=$ Diclytra canadensis (Goldie) DC.

D. cucullaria (L.) Bernh., Dutchman's Breeches $=$ Diclytra cucullaria (L.) DC.
Dichanthelium acuminatum (Sw.) Gould \& Clark $=$ Panicum acuminatum Sw.

Dichanthelium boreale (Nash) Freckmann $=$ Panicum boreale Nash

Dichanthelium clandestinum (L.) Gould = Panicum clandestinum L.

Dichanthelium commutatum $($ Schultes $)$ Gould $=$ Panicum commutatum Schultes

Dichanthelium depauperatum (Muhl.) Gould = Panicum depauperatum Muhl.

Dichanthelium dichotomum (L.) Gould = Panicum dichotomum L.

Dichanthelium latifolium (L.) Gould \& C. Clark = Panicum latifolium L.

Dichanthelium linearifolium (Scribn. ex Nash) Gould = Panicum linearifolium Scribn. ex Nash in Britt. \& Brown

Dichanthelium oligosanthes (J. A. Schultes) Gould var. scribnerianum (Nash) Gould $=$ Panicum oligosanthes J. A. Schultes var. scribnerianum (Nash) Fern.

Dichanthelium sphaerocarpon (Ell.) Gould var. sphaerocarpon $=$ Panicum sphaerocarpon Ell. var. sphaerocarpon

Dichanthelium villosissimum (Nash) Freckman var. praecocius (Hitchc. \& Chase) Freckmann $=$ Panicum villosissimum Nash

Dichanthelium xanthophysum (A. Gray) Freckman $=$ Panicum xanthophysum A. Gray

Diclytra canadensis (Goldie) DC. $=$ Dicentra canadensis (Goldie) Walp.

Diclytra cucullaria (L.) DC. $=$ Dicentra cucullaria (L.) Bernh.

DIERVILLA Mill. (Caprifoliaceae)

D. Ionicera Mill., Bush Honeysuckle $=$ D. trifida Moench

Diervilla trifida Moench $=$ Diervilla lonicera Mill .

DIGITARIA Heist. ex Fabr. (Poaceae)

D. cognata (Schultes) Pilger, Diffuse Crab-grass, Fall Witchgrass $=$ Leptoloma cognatum (Schultes) Chase

*D. ischaemum (Schreb. ex Schweig.) Schreb. ex Muhl., Small or Smooth Crab-grass $=\mathrm{D}$. ischaemum var. mississippiensis (Gatt.) Fern. $=$ Panicum glabrum (Schrad.) Gaud. 
$=$ Syntherisma ishaemum (Schreb. ex

Schweig.) Nash

*D. sanguinalis (L.) Scop., Large Crab-grass, Tall or Hairy Crab-grass

$=$ Digitaria adscendens (Kunth) Henr.

$=$ Panicum sanguinale $\mathrm{L}$.

$=$ Syntherisma sanguinalis (L.) Dulac

Dilepyrum erectum (Schreb) Farw. =

Brachyelytrum erectum (Schreb.) Beauv.

DIOSCOREA L. (Dioscoreaceae)

*D. batatas Dcne., Yam, Chinese Yam, Cinnamon-vine

$=$ D. oppositifolia L.

Dioscorea oppositifolia L. $=$ Dioscorea batatas

Dcne.

Dioscorea quaternata of old NY reports in part $=$ Dioscorea villosa L.

D. villosa L., Wild Yam, Yellow Yam

$=\mathrm{D}$. quaternata of old NY reports in part

Diphasiastrum digitatum (A. Br.) Holub $=$ Lycopodium digitatum Dill. ex A. Br.

Diphasiastrum digitatum $\times$ D. tristachyum $=$

Diphasiastrum $\times$ habereri (House) Holub $=$ Lycopodium digitatum $\times$ L. tristachyum $=$ Lycopodium $\times$ habereri House

Diphasiastrum tristachyum (Pursh) Holub = Lycopodium tristachyum Pursh

Diphasium tristachyum (Pursh) Rothm. = Lycopodium tristachyum Pursh

DIPLACHNE Beauv. (Poaceae)

*D. fascicularis (Lam.) Beauv., Clustered

Saltmeadow-grass, Sprangletop

$=$ Leptochloa fascicularis (Lam.) A. Gray

$=$ L. fusca (L.) Kunth. ssp. fascicularis

(Lam.) N. Snow

DIPLAZIUM Sw. (Dryopteridaceae)

Diplazium acrostichoides (Sw.) Butters $=$ Deparia acrostichoides (Sw.) Kato

D. pycnocarpon (Sprengel) M. Broun, Narrowleaved Spleenwort, Silvery Spleenwort, Silvery Glade-fern

$=$ Asplenium angustifolium (of authors)

$=$ A. pycnocarpon Sprengel

$=$ Athyrium angustifolium (Michx.) Milde

$=$ A. pycnocarpon (Sprengel) Tidestr.
Diplopappus umbellatus (Mill.) Hook. = Aster umbellatus Mill.

DIPLOTAXIS DC. (Brassicaceae)

*D. muralis (L.) DC., Sand Rocket, Wall-rocket $=$ Sisymbrium murale L.

*D. tenuifolia (L.) DC., Wall Rocket $=$ Sisymbrium tenuifolia (L.) DC.

DIPSACUS L. (Dipsacaceae)

*D. fullonum L., Common Teasel, Fuller's Teasel $=$ D. sylvestris Huds.

*D. laciniatus L., Cut-leaf Teasel

Dipsacus sylvestris Huds. $=$ Dipsacus fullonum L.

DIRCA L. (Thymelaeaceae)

D. palustris L., Leatherwood, Moosewood, Wicopy, Rope-bark

DISPORUM Salisb. ex D. Don (Liliaceae)

D. lanuginosum (Michx.) Nichols., Yellow Mandarin

$=$ Prosartes lanuginosa (Michx.) Don

Dodecatheon meadia (excluded by Zander \& Pierce 1979)

Doellingeria infirma (Michx.) Greene $=$ Aster infirmus Michx.

Doellingeria umbellata (P. Mill.) Nees var. umbellata $=$ Aster umbellatus P. Mill.

DRABA L. (Brassicaceae)

D. arabisans Michx., Rockcress Whitlow-grass

D. reptans (Lambert) Fernald, Whitlow-grass $=\mathrm{D}$. caroliniana Walt.

*D. verna L., Whitlow-grass $=$ Erophila verna (L.) Bess.

DRACOCEPHALUM L. (Lamiaceae)

Dracocephalum formosius (Lunell) Rydb. = Physostegia virginiana (L.) Benth.

D. parviflorum Nutt., Dragonhead

Dracocephalum speciosum Sweet $=$ Physostegia virginiana (L.) Benth.

Dracocephalum virginianum L. $=$ Physostegia virginiana (L.) Benth.

Dracontium foetidum L. $=$ Symplocarpus foetidus (L.) Salisb. ex Nutt.

DROSERA L. (Droseraceae) 
D. intermedia Hayne, Spatulate-leaved Sundew, Narrow-leaved Sundew

$=\mathrm{D}$. longifolia Michx. in part

Drosera longifolia Michx. in part $=$ Drosera intermedia Hayne

D. rotundifolia L., Round-leaved Sundew $=\mathrm{D}$. rotundifolia var. comosa Fern.

Drymocallis agrimonioides (Pursh) Rydb. = Potentilla arguta Pursh

DRYOPTERIS Adans. (Dryopteridaceae) Dryopteris austriaca var. fructuosa (Gilb.) Mort. = [Dryopteris carthusiana $\times$ intermedia $=\times$ triploidea Wherry]

Dryopteris austriaca var. intermedia (Muhl. ex Willd.) Morton $=$ Dryopteris intermedia (Muhl. ex Willd.) A. Gray

D. campyloptera (Kunze) Clarkson, Mountain Wood-fern

= Aspidium campyloptera Kunze

= D. austriaca of authors, not (Jacq.) Woyner

$=$ D. dilatata var. americana (Fisch. ex Kunze) Benedict

$=$ D. spinulosa var. americana (Fisch. ex Kunze) Fern.

$=$ Thelypteris dilatata (Hoffm.) House

= Thelypteris dilatata var. americana (Fisch.) House

No vouchered specimens occur for any western New York county other than post1980 specimens that exist for Cattaraugus (NYFA 1990). The fern occurs in Ontario (as D. expansa (C. Presl) Fraser-Jenkins \& Jermy (Morton \& Venn 1990). No specimens at BUF confirm the occurrence of this fern in our region and the peninsular region of Ontario. The treatment of this species by Montgomery and Wagner Jr. (FNA Vol. 2, 1993, p. 288) indicates the species does not occur in Ontario, and only in the Adirondack or Hudson River area of New York State (not western or central New York). The species should perhaps be excluded from our area.]

D. carthusiana (Vill.) Fuchs, Spinulose Shieldfern, Spinulose Wood-fern = D. austriaca var. spinulosa (Muell.) Fiori = D. spinulosa (Muell.) Watt
$=$ Polypodium carthusianum Vill.

$=$ Thelypteris spinulosa (O.F.Muell.) Nieuwl.

D. carthusiana $\times$ cristata $=\mathrm{D} . \times$ uliginosa $(\mathrm{A} . \mathrm{Br}$. ex Dowell) Druce, Wood-fern $=$ D. cristata $\times$ spinulosa

D. carthusiana $\times$ intermedia $=$ D. $\times$ triploidea Wherry, Wood-fern = D. austriaca var. fructuosa (Gilb.) Mort.

$=\mathrm{D}$. intermedia $\times$ spinulosa

D. celsa (Palmer) Knowlt., Palmer \& Poll., Logfern

D. celsa $\times$ goldiana, Log-fern

D. clintoniana (D. C. Eat. ex A. Gray) Dowell, Clinton's Fern, Clinton's Shield-fern

$=$ Aspidium cristatum var. clintonianum D. C. Eat.

$=$ Dryopteris cristata var. clintoniana $(D . C$. Eat. ex A. Gray) Underw.

$=$ Thelypteris clintoniana (D. C. Eaton ex A. Gray) House

D. clintoniana $\times$ goldiana Wood-fern

D. clintoniana $\times$ intermedia $=\mathrm{D} . \times$ dowellii (Farw.) Wherry, Dowell's Wood-fern

$=$ Thelypteris cristata var. clintoniana $\times \mathrm{T}$. spinulosa var. intermedia of Zenkert (1934).

D. cristata (L.) A. Gray, Crested Shield-fern, Crested Wood-fern

$=$ Aspidium cristatum (L.) Sw.

$=$ Polypodium cristatum $\mathrm{L}$.

$=$ Thelypteris cristata (L.) Nieuwl.

Dryopteris cristata var. clintoniana (D.C.Eat.) Underw. $=$ Dryopteris clintoniana (D. Eat. ex Gray) Dowell

D. cristata $\times$ intermedia $=$ D. $\times$ boottii (Tuckerm.) Underw., Boott's Wood-fern

= Aspidium boottii Tuckerm. of Zenkert, 1934.

Dryopteris cristata $\times$ spinulosa $=[$ Dryopteris carthusiana $\times$ cristata $=$ D. $\times$ uliginosa $(A$. Br. ex Dowell) Druce]

Dryopteris disjuncta (Ledeb.) Morton = Gymnocarpium dryopteris (L.) Newm.

D. goldiana (Hook. ex Goldie) Gray, Goldie's Fern, Giant Wood-fern

$=$ Aspidium goldianum Hook. ex Goldie

$=$ Thelypteris goldiana (Hook. ex Goldie) Nieuwl. 
D. goldiana $\times$ marginalis $=$ D. $\times$ neowherryi $W$. Wagner, Wood-fern

Dryopteris hexagonoptera (Michx.) Christ. = Phegopteris hexagonoptera (Michx.) Fée

D. intermedia (Muhl. ex Willd.) A. Gray, Common Wood-fern, Fancy-fern $=$ Aspidium intermedium Muhl. ex Willd.

= A. spinulosum var. intermedium (Muhl.) D.C. Eaton

$=$ Dryopteris austriaca var. intermedia (Muhl. ex Willd.) Morton

= D. spinulosa (O.F. Muell.) Watt var. concordiana (Davenport) Eastman

$=$ D. spinulosa var. intermedia (Muhl. ex Willd.) Underw.

$=$ Thelypteris intermedia (Muhl. ex Willd.) House

$=$ Thelypteris spinulosa var. intermedia (Muhl.) Weatherby

D. marginalis (L.) Gray, Marginal Shield-fern, Marginal Wood-fern

$=$ Aspidium marginale (L.) Sw.

$=$ Thelypteris marginalis (L.) Nieuwl.

Dryopteris phegopteris (L.) Christ. $=$ Phegopteris connectilis (Michx.) Watt

Dryopteris spinulosa (Muell.) Watt $=$ Dryopteris carthusiana (Vill.) Fuchs

Dryopteris spinulosa (O. F. Muell.) Watt var. concordiana (Davenport) Eastman $=$ Dryopteris intermedia (Willd.) A. Gray Dryopteris spinulosa var. intermedia (Muhl. ex Willd.) Morton $=$ Dryopteris intermedia (Muhl. ex Willd.) A. Gray

DULICHIUM L. Rich. ex Pers. (Cyperaceae)

D. arundinaceum (L.) Britt., Threeway Sedge = D. spathaceum (L.) Pers.

Dulichium spathaceum (L.) Pers. = Dulichium arundinaceum (L.) Britt.

\section{DYSSODIA Cav. (Asteraceae)}

Dyssodia chrysanthemoides (Willd.) Lag. = Dyssodia papposa (Vent.) Hitchc.

*D. papposa (Vent.) Hitchc., Fetid Marigold = Boebera papposa (Vent.) Rydb. ex Britt.

$=$ D. chrysanthemoides (Willd.) Lag.

$=$ Tagetes papposa Vent.

Eatonia obtusata (Michx.) Gray $=$ Sphenopholis obtusata (Michx.) Scribn.
Eatonia pennsylvanica A. Gray in part = Sphenopholis obtusata var. major (Torrey) Erdm.

Echinacea purpurea (L.) Moench (excluded by Zander \& Pierce 1979)

ECHINOCHLOA Beauv. (Poaceae)

*E. crus-galli (L.) Beauv., Japanese Millet, Barnyard-grass, Cock-spur Grass.

*ssp. crus-galli $=$ Panicum crus-galli L.

*ssp. edulis Hitchc., Japanese Millet, Billiondollar Grass.

$=$ E . crus-galli var. edulis Hitchc.

$=$ E. crus-galli var. frumentacea (Roxb.) Wight

$=$ E. frumentacea (Roxb.) Link

Echinochloa crus-galli var. hispidus Ell. = Echinochloa walteri (Pursh) Heller (not Nash ex Britt.)

Echinochloa crusgalli var. michauxii House $=$ Echinochloa muricata (Beauv.) Fern. var. muricata

Echinochloa frumentacea (Roxb.) Link = Echinochloa crus-galli ssp. edulis Hitchc.

Echinochloa microstachya (Wieg.) Rydb. = Echinochloa muricata var. microstachya Wieg.

E. muricata (Beauv.) Fern., Cockspur-grass, Barnyard-grass

var. microstachya Wieg. Small-spiked

Barnyard-grass

= E. microstachya (Wieg.) Rydb.

$=$ E. pungens var. microstachya (Weig.)

Fern.\& Grisc.

$=$ E. occidentalis (Wieg.) Rydb.

var. muricata, Rough Barnyard-grass

$=$ E . crusgalli var. michauxii House

$=$ E. pungens (Poir.) Rybd.

Echinochloa occidentalis (Wieg.) Rydb. = Echinochloa muricata var. microstachya Wieg.

Echinochloa pungens var. microstachya (Weig.) Fern.\& Grisc. $=$ Echinochloa muricata var. microstachya Wieg.

Echinochloa pungens (Poir.) Rydb. = Echinochloa muricata var. muricata (Beauv.) Fern.

E. walteri (Pursh) Heller (not Nash ex Britt.), Water-millet, Walter's Cockspur-grass 
ECHINOCYSTIS Torrey \& A. Gray

(Cucurbitaceae)

E. lobata (Michx.) Torr. \& A. Gray, Wild Cucumber, Prickly Cucumber, Wild Balsam-apple

$=$ Micrampelis lobata (Michx.) Greene

$=$ Sicyos lobata Michx.

ECHINOPS L. (Asteraceae)

*E. ritro L.

ssp. ruthenicus (Bieb.) Nyman, Globe Thistle

*E. sphaerocephalus L., Globe Thistle

Echinospermum lappula (L.) Lehm. = Lappula squarrosa (Retz.) Dumort.

ECHIUM L. (Boraginaceae)

*E. vulgare L., Viper's Bugloss, Blue-weed, Bluedevil

$=$ E. plantagineum of NY reports, not L.

$=$ E. pustulatum of NY reports, not Sibth. \& Sm.

ELEOCHARIS R. Br. (Cyperaceae)

E. acicularis (L.) R.\& S., Needle Spikerush, Hairgrass, Slender Rush

Eleocharis acuminata (Muhl.) Nees = Eleocharis elliptica Kunth var. elliptica

Eleocharis annua (Thuill.) House = Eleocharis obtusa var. ovata (Roth) Drap. \& Mohl.

Eleocharis calva Torr. $=$ Eleocharis erythropoda Steud.

Eleocharis capitata (of Zenkert, 1934) = Eleocharis elliptica Kunth var. elliptica

Eleocharis compressa Sulliv. $=$ Eliocharis elliptica Kunth var. elliptica

Eleocharis compressa var. atrata Svenson $=$ Eleocharis elliptica Kunth

Eleocharis diandra Wright $=$ Eleocharis obtusa (Willd.) Schultes var. obtusa

E. elliptica Kunth, Compressed Spikerush var. elliptica

$=$ E. acuminata (Muhl.) Nees

$=$ E. capitata $(\mathrm{L}$.$) R. Br.$

$=$ E. compressa Sulliv.

$=$ E. compressa var. atrata Svenson

$=$ E. tenuis of NY reports, not (Willd.)

Schultes

$=$ E. tenuis var. borealis (Svens.) Gleason
E. erythropoda Steud., Red-footed Spikerush = E. glaucescens (Willd.) R. \& S.

$=$ E. calva Torr.

E. flavescens (Poir.) Urban., Olive Spikerush

$=$ E. flavescens var. olivacea (Torrey)

Gleason

$=$ E. olivacea Torrey

Eleocharis glaucescens (Willd.) R. \& S. = Eleocharis erythropoda Steud.

E. intermedia Schultes (not Muhl.), Matted Spikerush

$=$ E. reclinata Kunth

$=$ E. reclinata var. habereri House

Eleocharis obtusa var. ellipsoides Fern. = Eleocharis obtusa (Willd.) Schultes var. obtusa

Eleocharis obtusa var. jejuna Fern. $=$ Eleocharis obtusa (Willd.) Schultes var. obtusa

E. obtusa (Willd.) Schultes, Spikerush, Fat Spikerush

var. obtusa

$=$ E. diandra Wright

$=$ E. obtusa var. ellipsoides Fern.

$=$ E. obtusa var. jejuna Fern.

var. ovata (Roth) Drap. \& Mohl., Ovate Spikerush

$=$ E. annua (Thuill.) House

= E. ovata (Roth) R. \& S.

Eleocharis olivacea Torrey $=$ Eleocharis flavescens (Poir.) Urban.

Eleocharis ovata (Roth) R. \& S. = Eleocharis obtusa var. ovata (Roth) Drap. \& Mohl.

E. palustris (L.) R.\& S., Creeping Spikerush $=$ E. palustris var. major Sonder

E. pauciflora (Lightf.) Link., Few-flowered Spikerush

$=$ E. quinqueflora var. fernaldii Svens.

$=$ Scirpus pauciflorus Lightf.

Eleocharis quinqueflora var. fernaldii Svens. = Eleocharis pauciflora (Lightf.) Link.

Eleocharis reclinata Kunth $=$ Eleocharis intermedia Schultes

Eleocharis reclinata var. habereri House $=$ Eleocharis intermedia Schultes

E. rostellata (Torr.) Torr., Beaked Spikerush

Eleocharis tenuis of NY reports, not (Willd.) Schultes $=$ Eleocharis elliptica Kunth var. elliptica 
Eleocharis tenuis var. borealis (Svenson) Gl. = Eleocharis elliptica Kunth

ELEUSINE Gaertn. (Poaceae)

*E. indica (L.) Gaertn., Goose-grass, Yard-grass, Wiregrass

ELODEA L. Rich. ex Michx. (Hydrocharitaceae)

E. canadensis Rich. ex Michx., Water-weed, Elodea, Ditch-moss

$=$ Anacharis canadensis (Rich. ex Michx.) Planch.

$=$ A. planchonii (Casp.) Rydb.

$=$ Apalanthe schweinitzii Planch.

$=$ Elodea planchonii Casp.

$=$ E. schweinitzii (Planch.) Casp.

$=$ Philotria canadensis (L. Rich. ex Michx.)

Britt.

$=$ P. occidentalis (Pursh) House

$=\mathrm{P}$. planchonii (Casp.) Rydb.

Elodea virginica (L.) Gray = Triadenum virginicum (L.) Raf.

ELYMUS L. (Poaceae)

E. canadensis L., Stout Nodding Wild Rye, Canada Wild Rye

Elymus canadensis L. var. wiegandii (Fern.) Bowd. $=$ Elymus wiegandii Fern.

Elymus caninus (L.) L. = Elymus trachycaulus (Link) Gould ex Shinners ssp. subsecundus (Link) Barkworth \& D. Dewey

E. hystrix L., Bottle-brush Grass

var. bigeloviana (Fern.) Bowden, Bigelow's Bottle-brush Grass

= Hystrix patula var. bigeloviana (Fern.) Deam

var. hystrix, Eastern Bottle-brush Grass

$=$ Asprella hystrix (L.) Willd.

$=$ Gymnostichum hystrix (L.) Schreb.

$=$ Hystrix patula Moench

Elymus repens (L.) Gould = Elytrigia repens (L.) Nevski

E. riparius Wieg., Riverbank Wild Rye, Marsh Wild Rye

Elymus striatus (of authors) $=$ Elymus villosus Muhl. ex Willd.

E. trachycaulus (Link) Gould ex Shinners, Slender Wheatgrass Rough-stemmed Wheatgrass ssp. subsecundus (Link) Barkworth \& D.

Dewey

$=$ Agropyron caninum (L.) Beauv.

$=$ A. trachycaulum var. glaucum (Pease \& Moore) Malte

$=$ A. trachycaulum var. majus (Vasey) Fern.

$=$ A. trachycaulum var. unilaterale (Vasey)

Malte

= Elymus caninus (L.) L.

$=$ Triticum caninum $\mathrm{L}$.

ssp. trachycaulus

= Agropyron caninum var. tenerum (Vasey)

Pease \& Moore

$=\mathrm{A}$. tenerum Vasey

$=$ A. trachycaulum (Link) Malte ex Lewis

$=$ A. trachycaulum var. novae-angliae (Scribn. ex Brainerd) Fern.

$=$ A. violaceum (Hornem.) Lange

E. villosus Muhl. ex Willd., Wild Rye

var. villosus

$=$ E. striatus (of authors)

E. virginicus L., Virginia Wild Rye var. virginicus

$=$ E. virginicus var. glabriflorus (Vasey) Bush

$=$ E. virginicus var. hirsutiglumis (Scribn.) Hitchc.

E. wiegandii Fern., Wiegand Wild Rye $=$ E. canadensis L. var. wiegandii (Fern.) Bowd.

ELYTRIGIA Desv. (Poaceae)

*E. repens (L.) Nevski, Quack-grass, Witch-grass, Quickgrass, Creeping Wild Rye

$=$ Agropyron repens (L.) Beauv.

$=$ A. repens var. littoreum Anderss.

$=$ A. repens var. nemorale Anderss.

$=$ A. repens var. pilosum Scribn.

= Elymus repens (L.) Gould

$=$ Triticum repens $\mathrm{L}$.

ENEMION Raf. (Ranunculaceae)

E. biternatum Raf., False Rue-Anemone = Isopyrum biternatum (Raf.) Torrey \& A. Gray

EPIFAGUS Nutt. (Orobanchaceae)

E. virginiana (L.) Bart., Beech-drops, Cancer-root $=$ Leptamnium virginianum (L.) Raf.

$=$ Orobanche virginiana $\mathrm{L}$. 
EPIGAEA L. (Ericaceae)

E. repens L., Trailing Arbutus

$=$ E. repens var. glabrifolia Fern.

EPILOBIUM L. (Onagraceae)

Epilobium adenocaulon Haussk. $=$ Epilobium ciliatum Raf. ssp. ciliatum

E. angustifolium L., Fireweed, Willow-herb, Wickup

$=$ Chamerion angustifolium (L.) Scop. ssp. circumvagum (Mosquin) Moldenke

$=$ Epilobium spicatum Lam.

E. ciliatum Raf., Northern Willow-herb, Hairy Willow-herb

ssp. ciliatum

$=$ E. adenocaulon Hausskn.

$=$ E. glandulosum Lehm. var. adenocaulon (Hausskn.) Fern.

$=$ E. glandulosum Lehm. var. perplexans (Trel.) Fern.

$=$ E. perplexans Trel.

ssp. glandulosum (Lehm.) Hoch \& Raven

= E. boreale Haussk.

$=$ E. glandulosum Lehm. var. glandulosum

$=$ E. glandulosum var. cardiophyllum Fern.

= E. glandulosum var. perplexans of authors, not (Trel.) Fern.

E. coloratum Biehler, Purple-leaved Willow-herb

$=$ E. tetragonum Pursh

Epilobium densum Raf. $=$ Epilobium strictum Muhl. ex Spreng.

Epilobium glandulosum var. adenocaulon

(Haussk.) Fern. = Epilobium ciliatum Raf. ssp. ciliatum

Epilobium glandulosum var. cardiophyllum Fern. $=$ Epilobium ciliatum ssp. glandulosum (Lehm.) Hoch \& Raven

Epilobium glandulosum Lehm. var. glandulosum $=$ Epilobium ciliatum Raf. ssp. glandulosum (Lehm.) Hoch \& Raven

Epilobium glandulosum var. occidentale (Trel.)

Fern. $=$ Epilobium ciliatum ssp. glandulosum (Lehm.) Hoch \& Raven

*E. hirsutum L., Hairy Willow-herb, European Fireweed

Epilobium lineare Muhl. $=$ Epilobium palustre L. Epilobium lineare LeConte $=$ Epilobium palustre L.
Epilobium molle Lam. $=$ Epilobium parviflorum Schreber

Epilobium molle Torr. $=$ Epilobium strictum Muhl. ex Spreng.

Epilobium oliganthum Michx. $=$ Epilobium palustre L.

E. palustre L., Marsh Willow-herb

$=$ E. lineare Muhl.

$=\mathrm{E}$. lineare LeConte

$=$ E. oliganthum Michx.

$=$ E. palustre var. monticola Haussk.

= E. palustre var. oliganthum (Michx.) Fern.

*E. parviflorum Schreber, Small-flowered Willow-herb $=$ E. molle Lam. not Torrey

Epilobium perplexans Trel. $=$ Epilobium ciliatum Raf. ssp. ciliatum

Epilobium spicatum Lam. $=$ Epilobium angustifolium $\mathrm{L}$.

E. strictum Muhl. ex Spreng., Soft Willow-herb $=$ E. densum Raf. $=$ E. molle Torr.

Epilobium tetragonum Pursh = Epilobium coloratum Biehler

EPIPACTIS Sw. (Orchidaceae)

*E. helleborine (L.) Crantz., Helleborine Orchid, Weed-orchid

$=$ E. latifolia (L.) All.

$=$ Serapias helleborine L.

Epipactis latifolia (L.) All. = Epipactis helleborine (L.) Crantz

Epipactis pubescens (Willd.) A.A. Eat. = Goodyera pubescens (Willd.) R.Br.

Epipactis repens var. ophoides (Fern.) A.A. Eat. = Goodyera repens var. ophoides Fern.

EQUISETUM L. (Equisetaceae)

E. arvense L., Common Horsetail, Field Horsetail $=\mathrm{E}$. arvense var. boreale Bong.

E. fluviatile L., Swamp Horsetail, Water-horsetail, Pipes, River-horsetail

$=$ E. limosum L.

= E. limosum var. verticillatum A. A. Eat.

Equisetum funstonii A. A. Eaton = Equisetum laevigatum A. Braun

E. hyemale L., Tall Scouring-rush

var. affine (Engelm.) A. A. Eat. $=$ E. hyemale var. elatum (Engelm.) Morton 
$=$ E. hyemale var. pseudohyemale (Farw.)

Morton

$=$ E. praealtum Raf.

$=\mathrm{E}$. robustum A. Br.

Equisetum hyemale var. intermedium A. A. Eat. =

[Equisetum hyemale $\times$ laevigatum $=\mathrm{E} . \times$ ferrisii Clute, see discussion under $\mathrm{E}$. laevigatum

E. hyemale $\times$ variegatum $=$ E. $\times$ mackii $($ Newm.$)$

Brich., Rough-tooth Scouring-rush, Hybrid Scouring-rush

$=\mathrm{E} . \times$ trachydon $\mathrm{R} . \mathrm{Br}$.

$=$ E. variegatum var. jesupii A. Eaton

Equisetum kansanum Schaffn. = Equisetum laevigatum A. Braun

E. laevigatum A. Braun, Smooth Scouring-rush $=$ E. funstonii A. A. Eaton

$=$ E. kansanum Schaffn.

Equisetum limosum L. = Equisetum fluviatile L.

Equisetum limosum var. verticillatum A.A.Eat. = Equisetum fluviatile L.

E. palustre L., Marsh-horsetail

$=$ E. palustre var. americanum Vict.

$=$ E. palustre var. ramosissimum Peck

Equisetum praealtum Raf. = Equisetum hyemale L. var. affine (Engelm.) A. A. Eaton

[Equisetum pratense Ehrh., report from Niagara Co. unsubstantiated by specimen (Young 1998).]

Equisetum robustum A. Br. = Equisetum hyemale L. var. affine (Engelm.) A. A. Eaton

E. scirpoides Michx., Dwarf Scouring-rush

E. sylvaticum L., Wood Horsetail

$=$ E. sylvaticum var. multiramosum (Fern.) Wherry

$=$ E. sylvaticum var. pauciramosum Milde

E. variegatum Schleich. ex Weber \& Mohr, Variegated Scouring-rush

ERAGROSTIS N. M. von Wolf (Poaceae)

E. capillaris (L.) Nees, Lacegrass

Eragrostis caroliniana (Spreng.) Scribn. = Eragrostis pectinacea (Michx.) Nees

*E. cilianensis (All.) Mosher (not Link or Hubb.), Strong-scented Love-grass, Stinkgrass, Skunkgrass, Snakegrass

$=$ E. major Host

$=$ E. megastachya (Koel.) Link
Eragrostis eragrostis (L.) Beauv. $=$ Eragrostis minor Host

E. frankii Meyer ex Steud., Frank's Love-grass, Lacegrass

*E. minor Host, Lovegrass

$=$ E. eragrostis (L.) Beauv.

$=$ E. poaeoides R. \& S.

Eragrostis multicaulis Steud. $=$ Eragrostis pilosa (L.) Beauv.

E. pectinacea (Michx.) Nees, Purple Lovegrass $=$ E. caroliniana (Spreng.) Scribn.

$=$ E. purshii Schrad.

Eragrostis peregrina Wieg. = Eragrostis pilosa (L.) Beauv.

*E. pilosa (L.) Beauv., India Lovegrass

$=$ E. multicaulis Steud.

$=$ E. peregrina Wieg.

Eragrostis poaeoides R. \& S. = Eragrostis minor Host

Eragrostis purshii Schrad. $=$ Eragrostis pectinacea (Michx.) Nees

E. spectabilis (Pursh) Steud., Purple Lovegrass, Tumblegrass

$=$ E. pectinacea of American authors, not

(Michx.) Nees

= E. spectabilis var. sparsihirsuta Farw.

ERECHTITES Raf. (Asteraceae)

E. hieracifolia (L.) Raf. ex DC., Pilewort, Fireweed

var. hieracifolia

$=$ E. hieracifolia var. intermedia Fern.

= E. hieracifolia var. praealta (Raf.) Fern.

Erechtites hieracifolia var. intermedia Fern. $=$ Erechtites hieracifolia (L.) Raf. ex DC.

Erechtites hieracifolia var. praealta (Raf.) Fern. $=$ Erechtites hieracifolia (L.) Raf. ex DC.

ERIGENIA Nutt. (Apiaceae)

E. bulbosa (Michx.) Nutt., Harbinger of Spring, Pepper-and-Salt

ERIGERON L. (Asteraceae)

Erigeron acris of authors, not L. $=$ Erigeron strigosus Muhl. ex Willd.

E. annuus (L.) Pers., Slender White-top, Daisyfleabane, Daisy Whitetop, White Scabious, Sweet Scabious 
Erigeron bellidifolius Muhl.ex Willd. = Erigeron pulchellus Michx.

Erigeron canadensis L. $=$ Conyza canadensis $(\mathrm{L}$. Cronq.

E. philadelphicus L., Philadelphia Fleabane $=$ E. philadelphicus var. provancheri (Vict. \& Rouss.) Boivin

E. pulchellus Michx., Robin's-plantain, Poor Robin's Plantain $=$ E. bellidifolius Muhl.ex Willd.

Erigeron ramosus (Walt.) BSP $=$ Erigeron strigosus Muhl. ex Willd.

E. strigosus Muhl. ex Willd., Daisy Fleabane $=\mathrm{E}$. acris of authors, not L.

$=$ E. ramosus (Walt.) BSP

$=$ E. strigosus varieties

ERIOCAULON L. (Eriocaulaceae)

E. aquaticum (Hill) Druce, Seven-angled Pipewort, White-buttons, Hatpins, Pipewort

$=$ E. articulatum (Huds.) Morong

$=$ E. pellucidum Michx.

$=$ E. septangulare With.

Eriocaulon pellucidum Michx. $=$ Eriocaulon aquaticum (Hill) Druce

Eriocaulon septangulare With. $=$ Eriocaulon aquaticum (Hill) Druce

ERIOCHLOA Kunth (Poaceae)

*E. acuminata (J. Presl) Kunth, Cup-grass $=\mathrm{E}$. lemmonii var. gracilis

ERIOPHORUM L. (Cyperaceae)

Eriophorum alpinum L. $=$ Scirpus hudsonianus (Michx.) Fern.

[Eriophorum angustifolium Honkeny was put in synonymy with Scirpus angustifolius (Honkey) T. Koyama by Zander and Pierce and excluded. Eriophorum angustifolium Honck. ssp. scabriusculum Hultén is reported in both New York State (Mitchell \& Tucker 1997) and Ontario (as E. triste (Th. Fries) Á. Löve \& Hadac, Morton \& Venn 1990). There is an unconfirmed report for Chautauqua Co. noted by Young (1998).]
Eriophorum callithrix of authors, not Cham. = Eriophorum vaginatum ssp. spissum (Fern.) Hultén

E. gracile Koch ex Roth, Slender Cotton-grass Eriophorum. polystachyon Torrey according to House 1924 = Eriophorum viridicarinatum (Engelm.) Fern.

[Eriophorum polystachyon var. angustifolium (Roth) Gray = Eriophorum angustifolium Roth (excluded) in ZP. in House angustifolium is Roth and Polyst. in Torr. not Honck or L. in M\&V, E. polystachyon is in part E. angustifolium and E. viridicarinatum p. 26]

Eriophorum spissum Fern. $=$ Eriophorum vaginatum L. ssp. spissum (Fern.) Hultén

E. tenellum Nutt., Rough Cotton-grass

E. vaginatum L., Hare's-tail ssp. spissum (Fern.) Hultén

$=$ E. spissum Fern.

$=$ E. callithrix of authors, not Cham.

E. virginicum L., Tawny Cotton-grass $=$ E. virginicum var. album A. Gray

E. viridicarinatum (Engelm.) Fern., Thin-leaved Cotton-grass

$=$ E. polystachyon Torrey according to House 1924

ERODIUM L'Hér. ex Ait. (Geraniaceae)

*E. cicutarium (L.) L'Hér. ex Ait., Storksbill, Pinclover, Alfileria

Erophila verna (L.) Bess. = Draba verna L.

ERUCA Mill. (Brassicaceae)

Eruca sativa Mill. $=$ Eruca vesicaria $($ L. $)$ Cav.

*E. vesicaria (L.) Cav., Rocket Salad, Gardenrocket

ssp. sativa (Mill.) Thell.

$=$ Brassica eruca L.

$=$ Eruca eruca (L.) Britt. ex Britt. \& Brown

$=$ E. sativa Mill.

ERUCASTRUM C. Presl (Brassicaceae)

*E. gallicum (Willd.) Schulz, Dog Mustard, French Rocket

$=$ E. pollichii Schimp. \& Spinn.

$=$ Sisymbrium erucastrum Poll.

$=\mathrm{S}$. gallicum Willd. 
Erucastrum pollichii Schimp. \& Spinn. = Erucastrum gallicum (Willd.) Schulz

ERYSIMUM L. (Brassicaceae)

Erysimum alliaria L. = Alliaria petiolata (Bieb.)

Cav. \& Grande

Erysimum barbarea L. = Barbarea vulgaris R. Br.

*E. cheiranthoides L., Wormseed Mustard, Treacle-mustard

$=$ Cheirinia cheiranthoides (L.) Link

Erysimum officinale L. $=$ Sisymbrium officinale (L.) Scop.

Erysimum vernum Mill. $=$ Barbarea verna (Mill. $)$ Aschers

Erythraea centaurium Pers. $=$ Centaurium erythraea Rafn. (not Raf.)

ERYTHRONIUM L. (Liliaceae)

E. albidum Nutt., White Adder's-tongue White Trout-lily, White Dog-tooth Violet

$=$ E. propullans of Ontario reports, not A. Gray (of Cameron, 1895)

E. americanum Ker, Yellow Adder's-tongue, Troutlily, Dog-tooth Violet

Evonymus (orth. var.) = Euonymus L.

EUONYMUS L. (Celastraceae)

E. americana L., Strawberry-bush, Bursting-heart "reject, Ontario report based on E. obovatus" (Morton \& Venn 1990).

Euonymus americana var. obovata (Nutt.) Torr. \& A. Gray = Euonymus obovata Nutt.

(*)E. atropurpurea Jacq., Wahoo, Burning Bush

*E. europaea L., Spindle-tree

*E. fortunei (Turcz.) Hand., Wintercreeper var. radicans (Sieb. ex Miq.) Rehd.

E. obovata Nutt., Running Strawberry-bush $=$ E. americana var. obovata Torrey \& A. Gray ex A. Gray

Eupatoriadelphus maculatus (L.) King \& H. Robinson $=$ Eupatorium maculatum L. var. maculatum

EUPATORIUM L. (Asteraceae)

Eupatorium ageratoides L.f. = Eupatorium rugosum Houtt.

E. altissimum L., Tall Thoroughwort
"Note: Ageratina altissima (L.) King \& H. Robinson is a synonym of E. rugosum" Mitchell \& Tucker (1997).

Eupatorium bruneri of NY authors, not A. Gray = Eupatorium maculatum L. var. maculatum Eupatorium bruneri var. foliosum (Fern.) House = Eupatorium maculatum L. var. foliosum (Fern.) Wieg.

Eupatorium falcatum Michx. = Eupatorium purpureum L.

E. fistulosum Barratt, Joe-Pye-weed, Trumpetweed

E. maculatum L., Joe-Pye-weed var. foliosum (Fern.) Wieg.

$=$ E. bruneri var. foliosum (Fern.) House

= E. maculatum var. bruneri (A. Gray) Breit., not Dougl.

= E. purpureum var. foliosum Fern.

var. maculatum

$=$ E. bruneri of NY authors, not A. Gray

= E. purpureum var. maculatum (L.) Darl.

$=$ Eupatoriadelphus maculatus (L.) King \& H. Robinson

$=$ Eutrochium maculatum (L.) E. Lamont

E. perfoliatum L., Boneset, Thoroughwort

E. purpureum L., Green-stemmed Joe-Pye-weed

$=$ E. falcatum Michx.

$=$ Eutrochium purpureum (L.) E. Lamont Eupatorium purpureum (of Day, 1882) was redetermined to be Eupatorium maculatum L. (Zander \& Pierce, 1979). Zenkert (1934, as E. falcatum Michx.) reports the species as "Rather rare. Open woods, edges of woods, in dry to

damp soil; sparse." Eaton and Schrot (1987) report this species, in Cattaraugus County, as "Frequent along roadsides edges of woods, meadows, alluvium of Allegheny River; abundant."

Eupatorium purpureum var. foliosum Fern. = Eupatorium maculatum L. var. foliosum (Fern.) Wieg.

Eupatorium purpureum var. maculatum (L.) Darl. = Eupatorium maculatum L. var. maculatum

E. rugosum Houtt., White Snakeroot, White Sanicle

= Ageratina altissima (L.) R. King \& H. Robinson 
$=$ Eupatorium ageratoides L.f.

$=$ E. urticaefolium Reich.

Eupatorium urticaefolium Reich. $=$ Eupatorium rugosum Houtt.

EUPHORBIA L. (Euphorbiaceae)

*E. corollata L., Flowering Spurge, Tramp's Spurge, Wild-hippo

*E. cyparissias L., Cypress Spurge

*E. dentata Michx., Toothed Spurge

$=$ Poinsettia dentata (Michx.) Klotzsch \& Garcke

*E. esula L., Leafy Spurge, Wolf's Milk

$=$ E. esula L. ssp. esula

= E. esula L. ssp. tommasiniana (Bertol.) Nyman

$=$ E. $\times$ pseudovirgata (Schur.) So

$=$ E. virgata Waldst. \& Kit.

*E. helioscopia L., Sun-spurge, Wartweed

Euphorbia hirsuta (Torr.) Weig. $=$ Chamaesyce vermiculata (Raf.) House

Euphorbia humistrata of NY reports, not Engelm. $=$ Chamaesyce maculata (L.) Small

Euphorbia hypericifolia of authors and Ontario reports $=$ Chamaesyce nutans (Lag.) Small

*E. lathyris L., Myrtle-spurge, Caper-spurge, Mole-plant, Mole-spurge

Euphorbia maculata L. = Chamaesyce maculata (L.) Small

Euphorbia maculata of authors in part not (L.) Small $=$ Chamaesyce nutans (Lag.) Small

Euphorbia nutans Lag. = Chamaesyce nutans (Lag.) Small

*E. peplus L., Petty Spurge

Euphorbia platyphylla of authors = Euphorbia platyphyllos L.

*E. platyphyllos L., Broad-leaved Spurge

$=$ E. platyphyllus and platyphylla of authors

Euphorbia polygonifolia L. $=$ Chamaesyce polygonifolia (L.) Small

Euphorbia preslii Guss. $=$ Chamaesyce nutans (Lag.) Small

Euphorbia supina Raf. $=$ Chamaesyce maculata (L.) Small

Euphorbia vermiculata Raf. $=$ Chamaesyce vermiculata (Raf.) House

EUPHRASIA L. (Scrophulariaceae)

*E. stricta Wolff ex Lehm., Eyebright
$=$ E. condensata Jord.

$=$ E. officinalis L.

$=$ E. rigidula Jord.

Eurybia divaricata (L.) Nesom $=$ Aster divaricatus L.

Eurybia macrophylla (L.) Cass. = Aster macrophyllus L. var. macrophullus

EUTHAMIA Nutt. ex Cass. (Asteraceae)

E. graminifolia (L.) Nutt. ex Cass., Narrow-leaved Goldenrod

$=$ Euthamia graminifolia var. nuttallii

(Greene) Stone

$=$ Solidago graminifolia (L.) Salisb.

$=\mathrm{S}$. graminifolia var. nuttallii (Greene) Fern.

$=\mathrm{S}$. graminifolia var. polycephala (Fern.)

Fern.

$=\mathrm{S}$. lanceolata L.

$=\mathrm{S}$. polycephala Fern.

Eutrochium maculatum (L.) E. Lamont var. maculatum $=$ Eupatorium maculatum L. var. maculatum

Eutrochium purpureum (L.) E. Lamont = Eupatorium purpureum L.

FAGOPYRUM Mill. (Polygonaceae)

*F. esculentum Moench, Buckwheat

= F. fagopyrum (L.) Karst.

$=$ F. sagittatum Gilib., a nom. rej.

$=\mathrm{F}$. vulgare Hill

Fagopyrum sagittatum Gilib. $=$ Fagopyrum esculentum Moench

Fagopyrum vulgare Hill = Fagopyrum esculentum Moench

FAGUS L. (Fagaceae)

Fagus ferruginea Ait. = Fagus grandifolia Ehrh.

F. grandifolia Ehrh., Beech, American Beech, Beechnut

$=\mathrm{F}$. ferruginea Ait.

$=$ F. grandifolia var. caroliniana (Loud.) Fern. \& Rehd.

Falcata comosa (L.) Kuntze = Amphicarpaea bracteata (L.) Rickett \& Stafleu

Fallopia cilinodis (Michx.) Holub. = Polygonum cilinode Michx.

Fallopia convolvulus (L.) Á. Löve = Polygonum convolvulus L. 
Fallopia dumetorum (L.) Holub. = Polygonum scandens L. var. dumetorum (L.) Gleason

Fallopia japonica (Hout.) Dene ssp. japonica $=$ Polygonum cuspidatum Sieb. \& Zucc.

Fallopia sachalinensis (Schmidt ex Maxim.) Dene. $=$ Polygonum sachalinensis F. Schmidt ex Maxim.

Fallopia scandens (L.) Holub. var. cristatum (Engelm. \& A. Gray) H. R. Hinds = Polygonum scandens L. var. cristatum (Engelm. \& A. Gray) Gleason

Fallopia scandens (L.) Holub. var. scandens $=$ Polygonum scandens L.

Fedia fagopyrum Torr. \& A. Gray = Valerianella chenopodifolia (Pursh) DC.

Fedia olitoria (L.) Vahl = Valerianella locusta (L.) Laterr. (not Betcke)

FESTUCA L. (Poaceae)

Festuca brachyphylla of NY report, not Schultes = Festuca filiformis Pourret

Festuca capillata Lam. $=$ Festuca filiformis Pourret

Festuca duriuscula of authors, not L. = Festuca trachyphylla (Hackel) Krajina

Festuca elatior L., a nomen confusum = Lolium pratense (Hudson) S. Darbyshire

*F. filiformis Pourret, Hair-fescue

$=\mathrm{F}$. brachyphylla of NY report, not Schultes

$=$ F. capillata Lam.

= F. ovina var. capillata (Lam.) Alef.

$=$ F. tenuifolia Sibth.

Festuca longifolia Thuill. $=$ Festuca trachyphylla (Hackel) Krajina

Festuca nutans (of authors) = Festuca subverticillata (Pers.) Alexe'ev

Festuca obtusa Biehler ex Spreng. = Festuca subverticillata (Pers.) Alexe'ev

Festuca octoflora Walt. = Vulpia octoflora (Walt.) Rydb.

Festuca ovina of some authors, not L. = Festuca trachyphylla (Hackel) Krajina

Festuca ovina var. capillata (Lam.) Alef. $=$ Festuca filiformis Pourret

Festuca ovina var. duriuscula (L.) Koch = Festuca trachyphylla (Hackel) Krajina

Festuca ovina var. hispidula Richt. = Festuca trachyphylla (Hackel) Krajina

Festuca pratensis Huds. = Lolium pratense (Hudson) S. Darbyshire
F. rubra L., Red Fescue

*ssp. fallax Thuill. Chewing's Fescue

$=$ F. rubra var. commutata Gaud.

*ssp. rubra

F. subverticillata (Pers.) Alexe'ev, Nodding

Fescue

$=$ F. obtusa Biehler

$=\mathrm{F}$. nutans (of authors)

Festuca tenella Willd. = Vulpia octoflora (Walt.)

Rydb. var. glauca (Nutt.) Fern.

Festuca tenuifolia Sibth. $=$ Festuca filiformis

Pourret

*F. trachyphylla (Hackel) Krajina, Sheep-fescue $=\mathrm{F}$. duriuscula of authors, not L.

$=$ F. longifolia Thuill.

$=\mathrm{F}$. ovina of some authors, not L.

$=$ F. ovina var. duriuscula (L.) Koch

$=$ F. ovina var. hispidula Richt.

Ficaria ficaria (L.) Karst. $=$ Ranunculus ficaria L.

FILIPENDULA P. Mill. (Rosaceae)

*F. ulmaria (L.) Maxim., Queen of the Meadow, Meadow-sweet, Whipped-cream, Spiraea

$=$ F. ulmaria var. denudata (J. \& C. Presl) Beck

Filix bulbifera (L.) Underw. = Cystopteris bulbifera (L.) Bernh.

Filix fragilis (L.) Gilib. = Cystopteris fragilis (L.) Bernh.

Fimbristylis capillaris A. Gray = Bulbostylis capillaris (L.) Clarke

FLOERKEA Willd. (Limnanthaceae)

F. proserpinacoides Willd., False Mermaid

FOENICULUM Mill. (Apiaceae)

*F. vulgare Mill., Fennel

$=$ Anethum foeniculum of Clinton's Journal 1862, Oct. 9

= F. foeniculum (L.) Karst.

FRAGARIA L. (Rosaceae)

Fragaria americana (Porter) Britt. $=$ Fragaria vesca ssp. americana (Porter) Staudt

Fragaria canadensis Michx., not Crantz = Fragaria virginiana Duchesne

Fragaria grayana Vilm. ex Gay $=$ Fragaria virginiana Duchesne 
Fragaria michauxiana House $=$ Fragaria virginiana Duchesne

Fragaria platypetala Rydb. $=$ Fragaria virginiana Duchesne

F. vesca L., Woodland Strawberry, Sow-teat Strawberry

ssp. americana (Porter) Staudt, American Wood Strawberry

$=$ F. americana (Porter) Britt.

$=$ F. vesca var. americana Porter

*ssp. vesca L. European Strawberry

F. virginiana Duchesne, Virginia Strawberry

$=\mathrm{F}$. canadensis Michx., not Crantz

$=\mathrm{F}$. grayana Vilm. ex Gay

$=\mathrm{F}$. michauxiana House

$=$ F. platypetala Rydb.

$=\mathrm{F}$. virginiana ssp. grayana (Vilm. ex Gay)

Staudt

$=$ F. virginiana ssp. platypetala (Rydb.)

Staudt

$=\mathrm{F}$. virginiana var. illinoensis (Prince) A. Gray

$=$ F. virginiana var. terra-novae (Rydb.) Fern.

Frangula alnus Mill. $=$ Rhamnus frangula $\mathrm{L}$.

FRASERA Walt. (Gentianaceae)

F. caroliniensis Walt., American Columbo, Green Gentian

$=$ Swertia caroliniensis (Walt.) Kuntze

FRAXINUS L. (Oleaceae)

F. americana L., White Ash, American Ash

= F. americana var. microcarpa A. Gray

*F. excelsior L., European Ash.

Fraxinus lanceolata Borkh. $=$ Fraxinus pennsylvanica Marsh.

F. nigra Marsh., Black Ash

$=$ Fraxinus sambucifolia Lam.

F. pennsylvanica Marsh., Red Ash, Green Ash

$=$ F. lanceolata Borkh.

$=\mathrm{F}$. pennsylvanica var. lanceolata (Borkh.) Sarg.

$=\mathrm{F}$. pennsylvanica var. subintegerrima

(Vahl) Fern.

$=\mathrm{F}$. pennsylvanica varieties

$=\mathrm{F}$. pubescens of American authors, not Lam.

$=\mathrm{F}$. quadrangulata of NY reports, not Michx. $=\mathrm{F}$. viridis Michx.f.
Fraxinus profunda Bush $=$ Fraxinus tomentosa Michx. f. (excluded by Zander \& Pierce 1979)

Fraxinus pubescens of American authors, not Lam. $=$ Fraxinus pennsylvanica Marsh.

Fraxinus quadrangulata of NY reports, not Michx. $=$ Fraxinus pennsylvanica Marsh.

Fraxinus sambucifolia Lam. $=$ Fraxinus nigra Marsh.

Fraxinus viridis Michx.f. $=$ Fraxinus pennsylvanica Marsh.

FROELICHIA Moench (Amaranthaceae)

*F. gracilis (Hooker) Moq., Cottonweed, Slender Snake-cotton

$=$ Oplotheca gracilis Hooker

FUIRENA Rottb. (Cyperaceae)

Fuirena hispida of NY reports, not Ell. = Fuirena pumila (Torr.) Spreng.

F. pumila (Torr.) Spreng., Umbrella-grass $=\mathrm{F}$. hispida of NY reports, not Ell.

$=\mathrm{F}$. squarrosa of NY reports, in large part, not Michx.

Fuirena squarrosa of NY reports, in large part, not Michx. $=$ Fuirena pumila (Torr.) Spreng.

FUMARIA L. (Fumariaceae)

Fumaria fungosa Ait. = Adlumia fungosa (Ait.) Greene ex BSP.

Fumaria recta Raf. $=$ Adlumia fungosa (Ait.) Greene ex BSP.

*F. officinalis L., Fumitory, Earth-smoke, Drug Fumitory

GAILLARDIA Foug. (Asteraceae)

*G. aristata Pursh, Blanket-flower

$=$ G. pulchella of some reports, not Foug.

GALACTIA P. Br. (Fabaceae)

Galactia glabella of NY reports, not Michx. = Galactia volubilis (L.) Britt.

Galactia regularis of NY reports, not (L.) BSP. = Galactia volubilis (L.) Britt.

G. volubilis (L.) Britt., Downy Milk-pea $=\mathrm{G}$. glabella of NY reports, not Michx.

$=$ G. regularis of NY reports, not (L.) BSP.

Gale palustris (Lam.) Chev. = Myrica gale L . 
GALEARIS Raf. (Orchidaceae)

G. spectabilis (L.) Raf., Showy Orchis

$=$ Galeorchis spectabilis (L.) Rydb. ex Britt.

$=$ Orchis spectabilis L.

GALEGA L. (Fabaceae)

G. officinalis L., Catgut, Goat's-rue

GALEOPSIS L. (Lamiaceae)

*G. tetrahit L., Hemp-nettle

*var. bifida (Boenn.) Lej. \& Court.

$=\mathrm{G}$. bifida Boenn.

$=$ G. tetrahit var. bifida (Boenn.) Lej. \& Court.

*var. tetrahit

Galeorchis spectabilis (L.) Rydb. ex Britt. = Galearis spectabilis (L.) Raf.

GALINSOGA Ruiz \& Pavón (Asteraceae)

Galinsoga bicolorata St. John \& White $=$ Galinsoga quadriradiata Ruiz \& Pavón

Galinsoga caracasana (DC.) Schultz $=$ Galinsoga

quadriradiata Ruiz \& Pavón

Galinsoga ciliata (Raf.) Blake = Galinsoga

quadriradiata Ruiz \& Pavón

*G. parviflora Cav., Peruvian Daisy, Galinsoga, Quickweed

*G. quadriradiata Ruiz \& Pavón, Peruvian Daisy, Quickweed, Gardener's-blight

$=\mathrm{G}$. bicolorata St. John \& White

$=$ G. caracasana (DC.) Schultz.

$=\mathrm{G}$. ciliata (Raf.) Blake

GALIUM L. (Rubiaceae)

(*)G. aparine L., Cleavers, Bedstraw, Goosegrass

G. asprellum Michx., Rough Bedstraw

G. boreale L., Northern Bedstraw

$=\mathrm{G}$. boreale varieties

Galium brandegei Gray in part $=$ Galium trifidum L. ssp. trifidum

Galium brevipes Fern. \& Wieg. = Galium trifidum L. ssp. trifidum

G. circaezans Michx., Wild Liquorice Bedstraw var. circaezans

$=\mathrm{G}$. circaezans var. glabrum Britt.

var. hypomalacum Fern.

Galium claytonii Michx. $=$ Galium tinctorium L.

Galium erectum Huds. $=$ Galium mollugo L .
G. labradoricum (Wieg.) Wieg., Northern Marshbedstraw, Cleavers

G. lanceolatum Torr., Torrey's Wild Liquorice Bedstraw

*G. mollugo L., Carpet-weed Bedstraw, False Baby's-breath, Wild Madder $=\mathrm{G}$. erectum Huds. = G. mollugo var. erectum (Huds.) Domin.

G. obtusum Bigel., Blunt-leaved Marsh-bedstraw $=\mathrm{G}$. tinctorium of some NY reports, not L.

*G. odoratum (L.) Scop., Sweet Woodruff $=$ Asperula odorata $\mathrm{L}$.

G. palustre L., Marsh-bedstraw, Ditch-bedstraw

G. pilosum Ait., Hairy Bedstraw

G. tinctorium L., Stiff Marsh-bedstraw $=\mathrm{G}$. claytonii Michx.

$=$ G. trifidum ssp. tinctorium (L.) Hara

$=$ G. trifidum var. tinctorium (L.) Torr. \& A. Gray

G. trifidum L., Small Bedstraw ssp. trifidum

$=\mathrm{G}$. brandegei Gray in part

$=\mathrm{G}$. brevipes Fern. \& Wieg.

Galium trifidum ssp. tinctorium (L.) Hara $=$ Galium tinctorium L.

Galium trifidum var. tinctorium (L.) Torr. \& Gray $=$ Galium tinctorium $\mathrm{L}$.

G. triflorum Michx., Sweet-scented Bedstraw $=$ G. triflorum var. asprelliforme Fern.

*G. verum L., Yellow Bedstraw

ssp. verum

GAULTHERIA L. (Ericaceae)

G. hispidula (L.) Muhl. ex Bigel., Creeping Snowberry, Moxie-plum, Maidenhairberry

= Chiogenes hispidula (L.) Torr. \& A. Gray

G. procumbens L., Wintergreen, Checker-berry, Teaberry, Mountain-tea, Ivory-leaf

GAURA L. (Onagraceae)

G. biennis L., Biennial Gaura

GAYLUSSACIA Kunth (Ericaceae)

G. baccata (Wang.) Koch, Black Huckleberry

= Andromeda baccata Wang

= G. resinosa (Ait.) Torr.\& A. Gray ex Torr. 
G. frondosa (L.) Torr. \& A. Gray ex Torr., Dangleberry, Dwarf or Blue Huckleberry, Blue-tangle

= Vaccinium frondosum $\mathrm{L}$.

Gaylussacia resinosa (Ait.) Torr.\& A. Gray ex

Torr. $=$ Gaylussacia baccata (Wang.) K. Koch

Genista scoparia (L.) Lam. = Cytisus scoparius (L.) Link

GENTIANA L. (Gentianaceae)

G. andrewsii Griseb., Closed Gentian, Bottlegentian

$=\mathrm{G}$. andrewsii fo. albiflora Britt.

G. clausa Raf., Blind Gentian, Closed Gentian, Bottle-gentian

Gentiana crinita Froel. $=$ Gentianopsis crinita (Froel.) Ma

Gentiana detonsa (of Day, 1882) $=$ Gentianopsis procera (Holm) Ma

G. linearis Froel., Narrow-leaved Gentian

Gentiana procera Holm $=$ Gentianopsis procera (Holm) Ma

Gentiana puberula of authors, not Michx. = Gentiana puberulenta Pringle

G. puberulenta Pringle, Downy Gentian

$=$ Dasystephana grayi of NY reports, not

(Kusn.) Britt.

$=\mathrm{G}$. puberula of authors, not Michx.

Gentiana quinquefolia L. $=$ Gentianella quinquefolia (L.) Small

G. saponaria L., Soapwort-gentian, Harvest-bells

GENTIANELLA Moench (Gentianaceae)

G. quinquefolia (L.) Small Gentian, Stiff Gentian, Ague-weed, Gall-of-the-Earth

$=$ Gentiana quinquefolia $\mathrm{L}$.

$=$ Gentianella quinquefolia ssp. occidentalis

(A. Gray) Gillett

GENTIANOPSIS Ma (Gentianaceae)

G. crinita (Froel.) Ma, Fringed Gentian $=$ Gentiana crinita Froel.

G. procera (Holm) Ma, Smaller Fringed Gentian

$=$ Gentiana detonsa (of Day, 1882)

$=$ Gentiana procera Holm

GERANIUM L. (Geraniaceae)

G. bicknellii Britt., Northern Crane's-bill
= G. bicknellii var. longipes (S. Wats.) Fern.

$=\mathrm{G}$. carolinianum var. longipes $\mathrm{S}$. Wats.

G. carolinianum L., Carolina Crane's-bill var. carolinianum

$=\mathrm{G}$. carolinianum var. confertiflorum Fern.

Geranium carolinianum var. longipes S. Wats. = Geranium bicknellii Britt.

*G. dissectum L., Cutleaved Crane's-bill

G. maculatum L.,Wild Crane's-bill, Spotted Geranium, Alumroot, Purple Cranesbill

*G. molle L., Dove's-foot Crane's-bill

Geranium pratense of authors, not L., and of Zenkert $(1934)=$ Geranium solanderi Carolin

*G. pusillum Burm.f. (not L.), Small-flowered Crane's-bill

G. robertianum L., Herb Robert

[*G. rotundifolium L., Round-leaved Crane's-bill, of Zenkert (1934), is excluded from the New York flora by Mitchell and Tucker (1997) as "old mis-ID's of G. bicknellii and G. sibericum."]

[*G. solanderi Carolin, of Zenkert, 1934, is excluded from the New York flora by Mitchell and Tucker (1997) as "old, incorrect reports"]

Gerardia flava L. = Aureolaria flava (L.) Farw. var. flava

Gerardia flava var. macrantha (Pennell) Fern. = Aureolaria flava var. macrantha Pennell

Gerardia integrifolia Gray = Aureolaria flava (L.) Farw.

Gerardia paupercula var. borealis (Pennell) Deam = Agalinis paupercula (Gray) Britt. var. borealis Pennell

Gerardia pedicularia L. $=$ Aureolaria pedicularia (L.) Raf.

Gerardia purpurea var. parviflora Benth. $=$ Agalinis paupercula (Gray) Britt. var. borealis Pennell

Gerardia quercifolia Pursh $=$ Aureolaria flava (L.) Farw. var. flava

Gerardia tenuifolia Vahl = Agalinis tenuifolia (Vahl.) Raf.

Gerardia virginica (L.) BSP. $=$ Aureolaria virginica (L.) Pennell

GEUM L. (Rosaceae) 
Geum agrimonioides Pursh $=$ Potentilla arguta Pursh

Geum album J.F. Gmel. = Geum canadense Jacq.

G. aleppicum Jacq., Yellow Avens $=$ G. allepicum ssp. strictum (Ait.) Clausen

$=\mathrm{G}$. aleppicum var. strictum (Ait.) Fern.

$=\mathrm{G}$. strictum Ait.

G. canadense Jacq., White Avens

$=$ G. album J. F. Gmel.

$=$ G. canadense var. camporum (Rydb.) Fern.

$=$ G. canadense var. grimesii Fern. \& Weath.

Geum carolinianum Walt. = Geum canadense Jacq.

Geum flavum (Porter) Bickn. $=$ Geum virginianum

L.

Geum hirsutum Muhl. = Geum virginianum L.

G. laciniatum Murray, Rough Avens, Herb-bennet $=\mathrm{G}$. laciniatum var. trichocarpum Fern.

= G. virginianum sensu House (1924), not L.

G. macrophyllum Willd., Large-leaved Avens

G. rivale L., Purple Avens, Chocolate-root, Wateravens

Geum strictum Ait. = Geum aleppicum Jacq.

*G. urbanum L., Town-avens

G. verum (Raf.) Torrey \& A. Gray, Spring-avens

G. virginianum L., Rough Avens, Herb-bennet

$=\mathrm{G}$. flavum (Porter) Bickn.

$=\mathrm{G}$. hirsutum Muhl.

Gilia linearis (Nutt.) Gray = Collomia linearis

Nutt.

Gillenia trifoliata (L.) Moench $=$ Porteranthus trifoliatus (L.) Britt.

Ginannia lanata (L.) Hubbard = Holcus lanatus L.

GLECHOMA L. (Lamiaceae)

*G. hederacea L., Gill-over-the-Ground, Ground Ivy

$=\mathrm{G}$. hederacea var. micrantha Moric.

$=\mathrm{G}$. hederacea var. parviflora (Benth.) House

$=$ Nepeta glechoma Benth.

$=$ N. hederacea $($ L. $)$ Trev.

GLEDITSIA L. (Fabaceae)

*G. triacanthos L., Honey-locust, Honey-shuck $=$ G. triacanthos var. inermis (L.) Schneid.

GL YCERIA R. Br. (Poaceae)

Glyceria aquatica (of authors) $=$ Glyceria grandis

S. Wats
[Glyceria arkansana Fern., Mannagrass = G. septentrionalis Hitchc. var. arkansana Steyerm. \& Kucera; reports unconfirmed by specimens for Cattaraugus, Chautauqua, Erie and Niagara Co. (Young 1998). "Note: There is a Sartwell specimen at Harvard (ca. 1840) labeled "western New York.” (Mitchell \& Tucker 1997). Reported by Mitchell and Tucker (1997) as extirpated from New York State.]

G. borealis (Nash) Batch-elder, Northern Mannagrass

$=$ Panicularia borealis Nash

G. canadensis (Michx.) Trin., Rattlesnake-grass

$=$ Panicularia canadensis (Michx.) Kuntze

Glyceria elongata (Torr.) Trin. = Glyceria melicaria (Michx.) Hubbard

Glyceria fluitans (L.) R.Br. (excluded by Zander \& Pierce 1979)

G. grandis S. Wats., Reed Meadow-grass

$=$ G. maxima (Hartm.) Holmb. ssp. grandis (S.Wats.) Hultén

$=$ Panicularia grandis (S. Wats.) Nash

Glyceria grandis $\mathrm{S}$. Wats. = Glyceria grandis $\mathrm{S}$.

Wats.

Glyceria maxima ssp. grandis (S. Wats.) Hultén = Glyceria grandis $\mathrm{S}$. Wats.

G. melicaria (Michx.) Hubbard, Long Mannagrass, Slender Manna-grass

$=$ G. elongata (Torr.) Trin.

$=$ Panicularia melicaria (Michx.) Hitchc.

Glyceria nervata (Willd.) Trin. = Glyceria striata (Lam.) Hitchc.

Glyceria pallida (Torr.) Trin. $=$ Torreyochloa pallida (Torr.) Church var. pallida

G. septentrionalis Hitchc., Floating Manna-grass

$=$ G. striata var. stricta (Scribn.) Fern.

$=$ Panicularia septentrionalis (Hitchc.) Bickn.

G. striata (Lam.) Hitchc., Nerved Manna-grass, Fowl Manna-grass

$=$ G. nervata (Willd.) Trin.

$=$ Panicularia nervata (Willd.) Kuntze

Glyceria striata var. stricta (Scribn.) Fern. =

Glyceria septentrionalis Hitchc.

Glycine bracteata L. = Amphicarpaea bracteata

(L.) Rickett \& Stafleu

Glycine apios L. = Apios americana Medik.

Glycine comosa L. = Amphicarpaea bracteata (L.) Rickett \& Stafleu 
GLYCYRRHIZA L. (Fabaceae)

G. lepidota Nutt. ex Pursh, Wild Liquorice

$=$ Liquivitia lepidota Nutt. ex Fras., a nomen illeg.

"Incorrect authority-citations for G. lepidota include: (Nutt.) Pursh and (Nutt.) Nutt. ex Pursh." Mitchell \& Tucker, 1997.

GNAPHALIUM L. (Asteraceae)

Gnaphalium decurrens Ives = Gnaphalium macounii Greene, according to House 1924

G. macounii Greene, Macoun's Cudweed, Everlasting

$=$ G. decurrens Ives according to House 1924; reported by Zenkert, 1934, p. 258.

= "Gnaphalium viscosum on NY reports, not Kunth, fide Cronquist, who saw the type specimens" (Mitchell \& Tucker, 1997).

$=$ Pseudognaphalium macounii (Greene) Kartesz

G. obtusifolium L., Fragrant Everlasting $=\mathrm{G}$. polycephalum Michx.

Gnaphalium polycephalum Michx. = Gnaphalium obtusifolium L.

G. purpureum L., Purple or Sweet Everlasting, Cudweed

$=$ Gamochaeta purpurea (L.) Cabrera

G. uliginosum L., Low Cudweed

$=$ Filaginella uliginosa $(\mathrm{L}$.) Opiz

"Gnaphalium viscosum on NY reports, not Kunth, fide Cronquist, who saw the type specimens" (Mitchell \& Tucker, 1997) = Gnaphalium macounii Greene

Gonolobus laevis (of authors) $=$ Cynanchum laeve (Michx.) Pers.

GOODYERA R. Br. (Orchidaceae)

G. pubescens (Willd.) R. Br., Downy Rattlesnakeplantain

$=$ Epipactis pubescens (Willd.) A. A. Eat.

$=$ Peramium pubescens (Willd.) MacM.

G. repens (L.) R. Br., Rattlesnake-plantain

$=$ Epipactis repens var. ophoides (Fern.) A.A. Eat.

$=$ Goodyera repens var. ophioides Fern.

$=$ Peramium repens (L.) Salisb.

$=$ Peramium secundum (Raf.) House
GRATIOLA L. (Scrophulariaceae)

G. neglecta Torr., Clammy Gratiola, Mud-hyssop

$=\mathrm{G}$. virginiana of some early NY reports, not L.

GRINDELIA Willd. (Asteraceae)

*G. squarrosa (Pursh) Dunal Gum-plant

*var. squarrosa

= G. nuda Wood

$=\mathrm{G}$. squarrosa var. nuda of authors, not

(Wood) A. Gray

Grossularia cynosbati (L.) Mill. = Ribes cynosbati

L.

Gymnadeniopsis clavellata (Michx.) Rydb. ex

Britt. $=$ Platanthera clavellata (Michx.)

Luer

GYMNOCARPIUM Newm. (Dryopteridaceae)

G. dryopteris (L.) Newm., Northern Oak-fern

$=$ Dryopteris disjuncta (Ledeb.) Morton

$=$ Phegopteris dryopteris (L.) Fée

= Polypodium dryopteris L.

$=$ Thelypteris dryopteris (L.) Slosson

GYMNOCLADUS Lamark (Fabaceae)

Gymnocladus canadensis Lam. $=$ Gymnocladus dioica (L.) K. Koch

(*)G. dioica (L.) K. Koch, Kentucky Coffee-tree $=\mathrm{G}$. canadensis Lam.

GYPSOPHILA L. (Caryophyllaceae)

*G. elegans Bieb., Showy Baby's Breath

Gymnostichum hystrix (L.) Schreb. = Elymus hystrix L. var. hystrix

Habenaria blephariglottis (Willd.) Hook. = Platanthera blephariglottis (Willd.) Lindl.

Habenaria blephariglottis var. holopetala (Lindl.)

A. Gray $=$ Platanthera blephariglottis (Willd.) Lindl.

Habenaria bracteata (Willd.) R.Br. = Coeloglossum viride (L.) Hartm.

Habenaria clavellata (Michx.) Spreng. = Platanthera clavellata (Michx.) Luer

Habenaria dilatata $($ Pursh $)$ Hook. $=$ Platanthera dilatata (Pursh) Lindl. ex Beck

Habenaria dilatata var. media (Rydb.) Ames $=$ Platanthera dilatata (Pursh) Lindl. ex Beck 
Habenaria fimbriata (Dryand. ex Ait.) R.Br. = Platanthera grandiflora (Bigel.) Lindl.

Habenaria flava var. herbiola (R. Br.) Ames \& Correll $=$ Platanthera flava $($ L. $)$ Lindl. var. herbiola (R. Br.) Luer

Habenaria flava var. virescens (Muhl. ex Willd.) Fern. $=$ Platanthera flava (L.) Lindl. var. herbiola (R.Br.) Luer

Habenaria herbiola R. Br. = Platanthera flava (L.) Lindl. var. herbiola (R. Br.) Luer.

Habenaria hookeri Torr. ex A. Gray = Platanthera hookeri (Torr. ex A. Gray) Lindl.

Habenaria hyperborea (L.) R. Br. = Platanthera hyperborea var. hyperborea (L.) Lindl.

Habenaria leucophaea (Nutt.) A. Gray = Platanthera leucophaea (Nutt.) Lindl.

Habenaria orbiculata (Pursh) Torr. $=$ Platanthera orbiculata var. orbiculata (Pursh) Lindl.

Habenaria peramoena of some NY reports, not A. Gray $=$ Platanthera psycodes (L.) Lindl. or P. grandiflora (Bigel.) Lindl.; see P. peramoena below.

Habenaria psycodes (L.) Spreng. = Platanthera psycodes (L.) Lindl.

Habenaria psycodes var. grandiflora (Bigel.) A. Gray $=$ Platanthera grandiflora (Bigel.) Lindl.

Habenaria virescens Muhl.ex Willd. = Platanthera flava (L.) Lindl. var. herbiola (R. Br.) Luer

Habenaria viridis (L.) R. Br. ex Ait. = Coeloglossum viride (L.) Hartm.

Habenaria viridis (L.) R. Br. var. bracteata (Muhl.) ex Willd.) A. Gray = Coeloglossum viride (L.) Hartm.

HACKELIA Opiz (Boraginaceae)

H. virginiana (L.) Johnst., Virginia Stickseed, Beggar-lice

$=$ Cynoglossum morisonii A. DC.

$=$ Lappula virginiana (L.) Greene

HAMAMELIS L. (Hamamelidaceae)

Hamamelis macrophylla Pursh $=$ Hamamelis virginiana $\mathrm{L}$.

H. virginiana L., Witch-hazel, Devil's Coffee

$=\mathrm{H}$. macrophylla Pursh

$=$ H. virginiana var. parviflora (Nutt.) Torr. \& A. Gray
HEDEOMA Pers. (Lamiaceae)

Hedeoma arkansana Nutt. $=$ Calamintha arkansana (Nutt.) Shinners

H. pulegioides (L.) Pers., American Pennyroyal, Mock-pennyroyal

HEDERA L. (Araliaceae)

*H. helix L., English Ivy

Hedyotis caerulea (L.) Hook. = Houstonia caerulea L.

Hedyotis longifolia (Gaertn.) Hook. = Houstonia longifolia Gaertn.

Hedysarum onobrychis L. = Onobrychis viciifolia Scop.

Heleborus trifolius L. = Coptis trifolia (L.) Salisb.

HELENIUM L. (Asteraceae)

H. autumnale L., Sneezeweed var. autumnale

$=\mathrm{H}$. autumnale var. canaliculatum (Lam.)

Torr. \& A. Gray

$=\mathrm{H}$. latifolium Mill.

*H. flexuosum Raf., Purple-headed Sneezeweed $=\mathrm{H}$. nudiflorum Nutt.

Helenium latifolium Mill. = Helenium autumnale L. var. autumnale

Helenium nudiflorum Nutt. $=$ Helenium flexuosum Raf.

HELIANTHEMUM Mill. (Cistaceae)

H. canadense (L.) Michx., Frostweed

$=$ Cistus canadensis $\mathrm{L}$.

$=$ Crocanthemum canadense $($ L.) Britt. ex

Britt. \& Brown

$=$ Helianthemum canadense var. sabulonum

Fern.

$=$ Lechea major $\mathrm{L}$.

HELIANTHUS L. (Asteraceae)

*H. annuus L., Common Sunflower, Mirasol

H. decapetalus L., Thin-leaf Sunflower $=\mathrm{H}$. scrophulariifolius Britt. $=\mathrm{H}$. trachelifolius Mill.

H. divaricatus L., Woodland Sunflower $=\mathrm{H}$. divaricatus var. angustifolius Kuntze

Helianthus doronicoides (of Day, 1882) = Helianthus tuberosus L.

H. giganteus L., Tall Sunflower 
$=\mathrm{H}$. giganteus var. subtuberosus (Bourgeau) Britt.

*H. grosseserratus Martens, Sawtoothed Sunflower

Helianthus laetiflorus Pers. = Helianthus pauciflorus Nutt. ssp. subrhomboideus (Rydb.) Spring \& Schilling

Helianthus laetiflorus var. rigidus Cass. $=$ Helianthus pauciflorus Nutt. ssp. subrhomboideus (Rydb.) Spring \& Schilling

Helianthus laetiflorus var. subrhomboideus (Rydb.) Fern.$=$ Helianthus pauciflorus Nutt. ssp. subrhomboideus (Rydb.) Spring \& Schilling

*H. pauciflorus Nutt., Prairie Sunflower ssp. subrhomboideus (Rydb.) Spring \& Schilling, Showy Sunflower

$=\mathrm{H}$. laetiflorus Pers.

$=\mathrm{H}$. laetiflorus var. rigidus Cass.

$=$ H. laetiflorus var. subrhomboideus (Rydb.) Fern.

$=\mathrm{H}$. rigidus (Cass.) Fern.

$=\mathrm{H}$. rigidus ssp. subrhomboides (Rydb.) Heiser

$=\mathrm{H}$. scaberrimus Ell.

$=$ H. subrhomboideus Rydb.

*H. pauciflorus ssp. subrhomboideus $\times$ tuberosus $=\mathbf{H} . \times$ laetiflorus Pers., Sunflower

$=$ Helianthus rigidus $\times$ tuberosus $=\mathrm{H} . \times$ laetiflorus

*H. petiolaris Nutt., Prairie Sunflower

Helianthus rigidus (Cass.) Fern. = Helianthus pauciflorus Nutt. ssp. subrhomboideus (Rydb.) Spring \& Schilling

Helianthus rigidus $\times$ tuberosus $=\mathrm{H} . \times$ laetiflorus $=$ [Helianthus pauciflorus ssp. subrhomboideus $\times$ tuberosus $=\mathrm{H} . \times$ laetiflorus Pers.]

Helianthus rigidus ssp. subrhomboides (Rydb.) Heiser $=$ Helianthus pauciflorus Nutt. ssp. subrhomboideus (Rydb.) Spring \& Schilling

Helianthus scaberrimus Ell. $=$ Helianthus pauciflorus Nutt. ssp. subrhomboideus (Rydb.) Spring \& Schilling

Helianthus scrophulariifolius Britt. = Helianthus decapetalus L.
H. strumosus L. Pale-leaved Sunflower, Wood Sunflower

Helianthus subrhomboideus Rydb. = Helianthus pauciflorus Nutt. ssp. subrhomboideus (Rydb.) Spring \& Schilling

Helianthus trachelifolius Mill. $=$ Helianthus decapetalus L.

*H. tuberosus L., Jerusalem Artichoke

$=\mathrm{H}$. doronicoides (of Day, 1882)

$=\mathrm{H}$. tuberosus var. subcanescens A. Gray

HELIOPSIS Pers. (Asteraceae)

H. helianthoides (L.) Sweet, False Sunflower, Oxeye

$=\mathrm{H}$. helianthoides ssp. scabra (Dunal) Fisch.

$=\mathrm{H}$. helianthoides var. scabra (Dunal) Fern.

$=\mathrm{H}$. laevis Pers.

$=$ H. laevis var. scabra (Dunal) Torr. \& A. Gray

$=$ H. scabra Dunal

Heliopsis laevis Pers. $=$ Heliopsis helianthoides (L.) Sweet

Heliopsis laevis var. scabra (Dunal) Torr. \& A. Gray $=$ Heliopsis helianthoides (L.) Sweet

Heliopsis scabra Dunal $=$ Heliopsis helianthoides (L.) Sweet

HEMEROCALLIS L. (Liliaceae)

*H. fulva (L.) L., Orange Day-lily

HEPATICA Mill. (Ranunculaceae)

Hepatica acutiloba DC. $=$ Hepatica nobilis Mill. var. acuta (Pursh) Steyerm.

Hepatica americana (DC.) Ker. = Hepatica nobilis Mill. var. obtusa (Pursh) Steyerm.

H. nobilis Mill., Liverleaf, Liverwort, Hepatica var. acuta (Pursh) Steyerm., Sharp-lobed Hepatica

$=$ Anemone acutiloba (DC.) Lawson

$=$ Hepatica acutiloba DC.

var. obtusa (Pursh) Steyerm., Blunt-lobed Hepatica

= A. hepatica (of authors)

$=$ H. americana (DC.) Ker.

$=$ H. triloba Chaix [

Hepatica triloba Chaix $=$ Hepatica nobilis Mill. var. obtusa (Pursh) Steyerm.

HERACLEUM L. (Apiaceae) 
*H. mantegazzianum Somm. \& Levier, Giant Hogweed

H. maximum Bartr., Cow-parsnip, Masterwort

$=\mathrm{H}$. lanatum Michx.

$=\mathrm{H}$. sphondylium var. montanum (Schleich. ex Gaud.) Briq.

Heracleum lanatum Michx. $=$ Heracleum maximum Bartr.

Heracleum sphondylium var. montanum (Schleich. ex Gaud.) Briq. = Heracleum maximum Bartr.

HESPERIS L. (Brassicaceae)

*H. matronalis L., Dame's Rocket, Dame's-violet, Mother-of-the-evening

$=\mathrm{H}$. matronalis ssp. cladotricha (Borbás) Hayek

HETERANTHERA Ruiz \& Pavón (Pontederiaceae)

H. dubia (Jacq.) MacM., Water-stargrass

$=$ Schollera graminea (Michx.) Raf.

$=$ Zosterella dubia (Jacq.) Small

Heterochloa schoenoides (L.) Host = Crypsis schoenoides (L.) Lam.

HIBISCUS L. (Malvaceae)

Hibiscus collinsiana Nutt. ex Torr. \& A. Gray = Hibiscus trionum L.

H. moscheutos L., Rose-mallow, Swamp-rose, Marsh-rose, Marsh-mallow, Wild Cotton

$=\mathrm{H}$. oculiroseus Britt.

$=\mathrm{H}$. opulifolius Greene

$=\mathrm{H}$. palustris $\mathrm{L}$.

Hibiscus oculiroseus Britt. $=$ Hibiscus moscheutos L.

Hibiscus opulifolius Greene $=$ Hibiscus moscheutos L.

Hibiscus palustris L. = Hibiscus moscheutos L.

*H. trionum L., Flower-of-an-hour

$=$ H. collinsiana Nutt. ex Torr. \& A. Gray

$=$ Trionum trionum (L.) Wooten \& Standl.

Hicoria alba of some American authors, not (L.)

Nutt. ex Ell. = Carya ovata (Mill.) K. Koch

Hicoria cordiformis (Wang.) Britt. ex Britt. \& Schafer $=$ Carya cordiformis (Wang.) K. Koch

Hicoria glabra (Mill.) Britt. = Carya glabra (Mill.) Sweet
Hicoria laciniosa (Michx. f.) Sarg. = Carya laciniosa (Michx. f.) G. Don

Hicoria ovalis (Wang.) Ashe = Carya ovalis (Wang.) Sarg.

Hicoria ovata (Mill.) Britt. = Carya ovata (Mill.) Koch

Hicoria ovata var. fraxinifolia (Sarg.) Ashe $=$ Carya ovata (Mill.) Koch

Hicoria ovata var. nuttallii (Sarg.) Ashe = Carya ovata (Mill.) Koch

Hicoria tomentosa (Poir. ex Lam.) Raf. = Carya tomentosa (Poir. ex Lam.) Nutt.

HIERACIUM L. (Asteraceae)

*H. aurantiacum L. Devil's Paint-brush, Orange Hawkweed

$=$ Pilosella aurantiaca (L.) F. W. Schultz \& Schultz-Bip.

*H. caespitosum Dumort., King-devil

$=\mathrm{H}$. pratense Tausch

$=$ Pilosella caespitosa (Dumort.) P. D. Sell. \& C. West.

$[$ Hieracium caespitosum $\times$ pilosella $=$ Hieracium $\times$ flagellare of authors, not Willd.] = Hieracium flagellare Willd.

Hieracium canadense Michx. = Hieracium kalmii L. var. kalmii

Hieracium canadense var. kalmii (L.) Scoggan $=$ Hieracium kalmii L. var. kalmii

*H. flagellare Willd., Hawkweed

$=\mathrm{H}$. caespitosum $\times$ pilosella $=\mathrm{H} . \times$ flagellare of authors, not Willd.

$=\mathrm{H}$. pilosella $\times$ pratense of authors

Hieracium florentinum All. $=$ Hieracium piloselloides Vill.

H. gronovii L., Hairy Hawkweed

H. gronovii $\times$ venosum $=\mathbf{H}$. $\times$ marianum Willd., Hybrid Hawkweed

$=$ H. marianum Willd. of Zenkert (1934)

$=\mathrm{H}$. traillii of NY reports, not Greene

H. kalmii L., Canada Hawkweed

var. kalmii

$=\mathrm{H}$. canadense Michx.

$=$ H. canadense var. kalmii (L.) Scoggan

$=\mathrm{H}$. umbellatum L.

Hieracium marianum Willd. of Zenkert (1934) =

$[$ Hieracium gronovii $\times$ venosum $=\mathrm{H} . \times$ marianum Willd.]

H. paniculatum L, . Panicled Hawkweed 
*H. pilosella L., Mouse-ear Hawkweed $=$ Pilosella officinarum F. W. Schultz \& Schultz-Bip.

Hieracium pilosella $\times$ pratense of authors $=$ Hieracium flagellare Willd.

*H. piloselloides Vill., King-devil $=\mathrm{H}$. florentinum All. = Pilosella piloselloides (Vill.) Soják

Hieracium pratense Tausch $=$ Hieracium caespitosum Dumort.

H. scabrum Michx., Rough Hawkweed Hieracium umbellatum L. = Hieracium kalmii L. var. kalmii

H. venosum L., Rattlesnake-weed, Poor Robin's Plantain $=$ H. venosum var. nudicaule (Michx.) Farw.

HOLCUS L. (Poaceae)

Holcus halepensis L. $=$ Sorghum halepense (L.) Pers.

*H. lanatus L., Velvet-grass

= Ginannia lanata (L.) Hubbard

$=$ Notholcus lanatus (L.) Nash ex Hitchc. in Jepson

Holcus sorghum L. = Sorghum bicolor (L.) Moench

Homalocenchrus oryzoides (L.) Poll. = Leersia oryzoides (L.) Sw.

Homalocenchrus virginicus (Willd.) Britt. = Leersia virginica Willd.

HORDEUM L. (Poaceae)

Hordeum distichon L. "are usually considered as races of Hordeum vulgare" L., according to House 1924 p. 128

*H. jubatum L., Squirrel-tail Grass, Foxtail-barley

*H. vulgare L., Barley, Pearl-barley

$=$ H. distichon L., according to House 1924 p. 128

$=\mathrm{H}$. vulgare var. trifurcatum (Schlect.) Alef.

HOUSTONIA L. (Rubiaceae)

H. caerulea L., Bluets, Quaker-ladies, Innocence $=$ Hedyotis caerulea (L.) Hook.

$=$ Houstonia coerulea L.

= Oldenlandia caerulea (L.) A. Gray

Houstonia canadensis on NY reports, not Willd. ex R. \& S. = Houstonia longifolia Gaertn.
Houstonia ciliolata Torr. $=$ Houstonia longifolia Gaertn.

Houstonia coerulea L. = Houstonia caerulea L.

H. longifolia Gaertn., Fringed Houstonia, Pale Bluets

$=$ Hedyotis longifolia (Gaertn.) Hook.

$=$ Houstonia canadensis of NY reports, not Willd. ex R. \& S.

$=\mathrm{H}$. ciliolata Torr.

HUDSONIA L. (Cistaceae)

H. tomentosa Nutt., Woolly Hudsonia, Hudsonia, Poverty-grass, False-heather, Beachheather

$=\mathrm{H}$. tomentosa var. intermedia Peck, not $\mathrm{H}$. ericoides $\times$ tomentosa

HUMULUS L. (Cannabaceae):

*H. japonicus Sieb. \& Zucc., Japanese Hops

H. lupulus L., Hops, Common Hop, Brewer's Hops

$=\mathrm{H}$. lupulus varieties

HUPERZIA Bernh. (Lycopodiaceae)

H. lucidula (Michx.) Trev., Shining Club-moss

$=$ Lycopodium lucidulum Michx.

= Lycopodium lucidulum var. occidentale (Clute) Wilson

$=$ Lycopodium lucidulum var. tryonii Mohlenbr.

HYBANTHUS Jacq. (Violaceae)

H. concolor (Forst.) Spreng., Green Violet $=$ Cubelium concolor (Forst.) Raf. $=$ Ionidium concolor (Forst.) Benth.

$=$ Solea concolor Forst.

HYDRASTIS L. (Ranunculaceae)

H. canadensis L., Goldenseal, Orange-root, Yellow Puccoon $=$ Warnera canadensis (L.) Mill.

HYDROCOTYLE L. (Apiaceae)

H. americana L., Water-pennywort, Navelwort

H. ranunculoides L.f., Floating Pennywort

H. verticillata Thunb. var. fetherstoniana (Jennings) Mathias

Only known from the type locality in Wyoming Co., an apparent endemic. 
HYDROPHYLLUM L. (Hydrophyllaceae)

Hydrophyllum appendiculatum of old NY reports, not Michx. = Hydrophyllum virginianum L.

H. canadense L., Broad-leaved Waterleaf, Canada Waterleaf

H. virginianum L. Virginia Waterleaf, Shawneesalad, Indian-salad, John's-cabbage

$=\mathrm{H}$. appendiculatum of old NY reports, not Michx.

Hylotelephium telephium (L.) H. Ohba. = Sedum telephium L.

HYOSCYAMUS L. (Solanaceae)

*H. niger L., Black Henbane, Potelée, Careillade

HYPERICUM L. (Clusiaceae)

H. ascyron L., Great St. John's-wort

$=\mathrm{H}$. macrocarpum Michx.

$=\mathrm{H}$. pyramidatum Ait.

H. boreale (Britt.) Bickn., Northern Dwarf St. John's Wort

$=$ Hypericum mutilum L. ssp. boreale (Britt.) Gillett

H. canadense L., Canada St. John's-wort

Hypericum corymbosum Muhl. ex Willd. = Hypericum punctatum Lam.

H. ellipticum Hook., Pale St. John's-wort

H. hypericoides (L.) Crantz, St. Andrew's Cross ssp. multicaule (Michx. ex Willd.) Robson

$=\mathrm{H}$. multicaule Michx. ex Willd.

H. kalmianum L., Kalm's St. John's-wort

Hypericum macrocarpum Michx. = Hypericum ascyron $\mathrm{L}$.

H. majus (A. Gray) Britt., Large St. John's-wort

Hypericum multicaule Michx. ex Willd. = Hypericum hypericoides (L.) Crantz ssp. multicaule (Michx. ex Willd.) Robson

H. mutilum L., Dwarf St. John's-wort $=$ H. mutilum var. parviflorum (Willd.) Fern.

Hypericum mutilum L. ssp. boreale (Britt.) Gillett $=$ Hypericum boreale (Britt.) Bickn.

*H. perforatum L., Common St. John's-wort, Klamath-weed, Goat-weed

H. prolificum L., Shrubby St. John's-wort $=$ H. spathulatum (Spach) Steud.

H. punctatum Lam., Spotted St. John's-wort $=\mathrm{H}$. corymbosum Muhl. ex Willd.
Hypericum pyramidatum Ait. $=$ Hypericum ascyron L.

Hypericum spathulatum (Spach) Steud. = Hypericum prolificum L.

Hypericum virginicum L. = Triadenum virginicum (L.) Raf.

Hypericum virginicum var. fraseri (Spach) Fern. = Triadenum fraseri (Spach) Gleason

HYPOCHAERIS L. (Asteraceae)

*H. radicata L., Cat's-ear

Hypopitys americana (DC.) Small = Monotropa hypopithys L.

Hypopitys lanuginosa (Michx.) Raf., not Nutt. = Monotropa hypopithys L.

Hypopitys multiflora var. americana DC. $=$ Monotropa hypopithys L.

HYPOXIS L. (Amaryllidaceae)

H. hirsuta (L.) Cov., Yellow Star-grass $=$ H. leptocarpa (Engelm. \& A. Gray) Small

Hypoxis leptocarpa (Engelm. \& A. Gray) Small = Hypoxis hirsuta (L.) Cov.

HYSSOPUS L. (Lamiaceae)

*H. officinalis L., Hyssop

Hystrix patula Moench = Elymus hystrix L. var. hystrix

Hystrix patula var. bigeloviana (Fern.) Deam $=$ Elymus hystrix L. var. bigeloviana (Fern.) Bowden

Ibidium plantagineum Rafinesque sec. Macdonald $1990=$ Spiranthes lucida (H. Eat.) Ames

ILEX L. (Aquifoliaceae)

Ilex ambigua var. montana (Torr. \& A. Gray) Ahles $=$ Ilex montana Torr. \& A. Gray ex A. Gray

I. montana Torr. \& A. Gray ex A. Gray, Largeleaved Holly

= I. ambigua var. montana (Torr. \& A. Gray) Ahles

$=$ I. montana var. mollis (A. Gray) Britt.

$=$ I. monticola A. Gray

Ilex monticola Gray = Ilex montana Torr. \& A. Gray ex A. Gray

I. verticillata (L.) A. Gray, Winterberry, Black Alder $=$ I. verticillata var. cyclophylla B. Robinson 
= I. verticillata var. padifolia (Willd.) Torr. \& A. Gray

$=$ I. verticillata var. tenuifolia Torr.

$=$ Prinos verticillatus $\mathrm{L}$.

Ilysanthes dubia (L.) Barnh. = Lindernia dubia (L.)

Pennell var. dubia

Ilysanthes dubia var. riparia (Raf.) Fern. =

Lindernia dubia (L.) Pennell var. dubia

Ilysanthes dubia var. inundata (Pennell) Pennell =

Lindernia dubia (L.) Pennell var. dubia

Ilysanthes gratioloides (L.) Benth. in DC. =

Lindernia dubia (L.) Pennell var. dubia

IMPATIENS L. (Balsaminaceae)

*I. balsamina L., Balsam, Garden-balsam

Impatiens biflora Walt., not Willd. = Impatiens capensis Meerb.

I. capensis Meerb., Spotted Touch-me-not, Spotted Jewelweed, Snapweed

$=$ I. biflora Walt., not Willd.

$=$ I. fulva Nutt.

= I. noli-tangere L. ssp. biflora (Walt.) Hultén

Impatiens fulva Nutt. = Impatiens capensis Meerb.

Impatiens noli-tangere L. ssp. biflora (Walt.)

Hultén = Impatiens capensis Meerb.

I. pallida Nutt., Pale Touch-me-not, Pale Jewelweed, Snapweed

INULA L. (Asteraceae)

*I. helenium L., Elecampane

Ionidium concolor (Forst.) Benth. $=$ Cubelium concolor (Forst.) Raf.

IPOMOEA L. (Convolvulaceae)

I. pandurata (L.) Meyer, Man-of-the-Earth, Wild Potato-vine

*I. purpurea (L.) Roth, Common or Garden Morning-glory $=$ Pharbitis purpurea (L.) Voigt

IRIS L. (Iridaceae)

*I. pseudacorus L., Yellow Iris, Yellow Flag

*I. sibirica L., Sibirian or Russian Iris

I. versicolor L., Blue Flag, Wild Iris, Poison Flag

I. virginica L., Southern Blue Flag

var. schrevii (Small) Anders.

$=$ I. schrevii Small

= I. versicolor var. shrevei (Small) Boivin
Isanthus brachiatus (L.) BSP. = Trichostema brachiatum $\mathrm{L}$.

Isanthus coeruleus Michx. $=$ Trichostema brachiatum L.

ISOETES L. (Isoëtaceae)

Isoëtes braunii Durieu = Isoëtes echinospora Durieu

I. echinospora Durieu, Spiny-spored Quillwort

$=\mathrm{I}$. braunii Durieu

= I. echinospora ssp. muricata (Durieu) Á. \& D. Löve

$=\mathrm{I}$. echinospora var. braunii (Durieu)

Engelm. ex A. Gray

$=\mathrm{I}$. muricata Durieu

I. engelmannii $\mathrm{A}$. $\mathrm{Br}$.

$=$ I. valida (Engelm.) Clute

I. lacustris $\times$ tuckermanii $=$ I. $\times$ heterospora A. Eaton

$=\mathrm{I}$. macrospora $\times$ tuckermanii

Isoetes macrospora $\times$ tuckermanii $=[$ Isoëtes lacustris $\times$ tuckermanii $=$ I. $\times$ heterospora A. Eaton]

Isoëtes muricata Durieu $=$ Isoëtes echinospora Durieu

Isoetes valida (Engelm.) Clute $=$ Isoetes engelmannii $\mathrm{A}$. Br.

Isopyrum biternatum (Raf.) Torrey \& A. Gray = Enemion biternatum Raf.

ISOTRIA Raf. (Orchidaceae)

I. verticillata (Muhl. ex Willd.) Raf., Five-leaved Orchid, Large Whorled Pogonia, Fiveleaves

= Pogonia verticillata (Muhl. ex Willd.) Nutt.

IVA L. (Asteraceae)

*I. xanthifolia Nutt., Burweed Marsh-elder

Jacksonia trifoliata Raf. = Polanisia dodecandra (L.) DC.

JEFFERSONIA B. Barton (Berberidaceae)

J. diphylla (L.) Pers., Twinleaf, Papoose-root, Rheumatism-root

JUGLANS L. (Juglandaceae)

J. cinerea L., Butternut, White Walnut

J. nigra L., Black Walnut 
Juncoides carolinae (S. Wats.) Kuntze = Luzula acuminata Raf.

Juncoides intermedium (Thuill.) Rydb. = Luzula campestris (L.) DC. in Lam. \& DC. var. multiflora (Retz.) Lej.

JUNCUS L. (Juncaceae)

J. acuminatus Michx., Sharp-fruited Rush

$=\mathrm{J}$. acuminatus var. legitimus Engelm. ex A. Gray

$=\mathrm{J}$. pallescens Meyer

$=\mathrm{J}$. paradoxus Meyer

$=\mathrm{J}$. pondii Wood

Juncus acuminatus of American authors, not

Engelm. or Michx. = Juncus brevicaudatus (Engelm.) Fern. p.p.

Juncus acuminatus var. debilis (A. Gray) Engelm. $=$ Juncus debilis A. Gray

Juncus affinis $\mathrm{R}$. Br. = Juncus alpinoarticulatus Chaix ex Vill. ssp. americanus (Farw.) Hämet-Ahti

J. alpinoarticulatus Chaix ex Vill., Alpine Rush ssp. americanus (Farw.) Hämet-Ahti

$=\mathrm{J}$. affinis $\mathrm{R}$. Br.

$=\mathrm{J}$. alpinus Vill. var. fuscescens Fern.

$=\mathrm{J}$. alpinus var. insignis Fries ex Engelm.

$=\mathrm{J}$. alpinus var. rariflorus of authors, not

Hartm.

$=\mathrm{J}$. articulatus var. pelocarpus (of Day, 1882)

Juncus alpinus Vill. var. fuscescens Fern. = Juncus alpinoarticulatus Chaix ex Vill. ssp. americanus (Farw.) Hämet-Ahti

Juncus alpinus var. insignis Fries ex Engelm. = Juncus alpinoarticulatus Chaix ex Vill. ssp. americanus (Farw.) Hämet-Ahti

Juncus alpinus var. rariflorus of authors, not Hartm. = Juncus alpinoarticulatus Chaix ex Vill. ssp. americanus (Farw.) Hämet-Ahti

Juncus arcticus ssp. littoralis (Engelm.) Hultén = Juncus balticus Willd. var. littoralis Engelm.

J. articulatus L., Jointed Rush

$=\mathrm{J}$. articulatus var. obtusatus Engelm.

$=\mathrm{J}$. articulatus var. stolonifer (Wohl.) House

Juncus articulatus var. pelocarpus (of Day, 1882) = Juncus alpinoarticulatus Chaix ex Vill. ssp. americanus (Farw.) Hämet-Ahti

J. balticus Willd., Baltic Rush var. littoralis Engelm.

$=\mathrm{J}$. arcticus ssp. littoralis (Engelm.) Hultén

$=\mathrm{J}$. balticus var. littoralis Engelm.

$=\mathrm{J}$. litorum Rydb.

Juncus bicornis Michx. = Juncus tenuis Willd. Juncus bicornis var. williamsii (Fern.) Vict. = Juncus tenuis Willd.

Juncus biflorus Ell. = Juncus marginatus Rostk.

J. brachycephalus (Engelm.) Buch., Short-headed Rush

$=\mathrm{J}$. canadensis var. brachycephalus Engelm. $=\mathrm{J}$. polycephalus var. depauperatus Torr.

J. brevicaudatus (Engelm.) Fern., Narrowpanicled Rush, Narrow-tailed Rush $=\mathrm{J}$. acuminatus of American authors, not Engelm. or Michx.

$=\mathrm{J}$. canadensis var. coarctatus Engelm.

J. bufonius L., Toad-rush

Juncus bulbosus bulbosus of authors including L. (1762), not L. (1753) = Juncus gerardii Loisel

Juncus bulbosus var. gerardii (Loisel.) A. Gray = Juncus gerardii Loisel.

J. canadensis J. Gay ex LaHarpe, Canada Rush $=\mathrm{J}$. canadensis var. longicaudatus Engelm.

$=\mathrm{J}$. canadensis var. sparsiflorus Fern.

$=\mathrm{J}$. longicaudatus (Engelm.) Mackz.

Juncus canadensis var. brachycephalus Engelm. = Juncus brachycephalus (Engelm.) Buch.

Juncus canadensis var. coarctatus Engelm. = Juncus brevicaudatus (Engelm.) Fern.

Juncus canadensis var. subcaudatus Engelm. = Juncus subcaudatus (Engelm.) Cov.\& Blake

Juncus compressus of NY reports, not Jacq. = Juncus gerardii Loisel.

Juncus conglomeratus L. = Juncus effusus L. var. conglomeratus (L.) Engelm. ex A. Gray

J. debilis A. Gray, Weak Rush $=\mathrm{J}$. acuminatus var. debilis (A. Gray) Engelm. ex A. Gray $=\mathrm{J}$. diffusissimus of NY reports, not Buckley

J. dudleyi Wieg., Dudley's Rush $=\mathrm{J}$. tenuis Willd. var. dudleyi (Wieg.) F. Hermann ex I. Johnston $=\mathrm{J}$. tenuis Willd. var. uniflorus (Farw.) Farw.

J. effusus L., Common Rush

var. conglomeratus (L.) Engelm. ex A. Gray $=\mathrm{J}$. conglomeratus L. 
$=\mathrm{J}$. effusus var. compactus of authors, not Lej. \& Court.

$=\mathrm{J}$. leersii T. Marsson

var. pylaei (LaHarpe) Fern. \& Wieg.

var. solutus Fern. \& Wieg.

= J. effusus ssp. solutus (Fern. \& Wieg.)

Maemet-Ahti

$=\mathrm{J}$. effusus var. solutus Fern. \& Wieg.

J. gerardii Loisel., Black-grass, Blackfoot-rush

$=\mathrm{J}$. bulbosus of authors including L. (1762), not L. (1753)

$=\mathrm{J}$. bulbosus var. gerardii (Loisel.) A. Gray

$=\mathrm{J}$. compressus of NY reports, not Jacq.

*J. inflexus L., European Meadow-rush (BUF Niagara Co. )

Juncus intermedius Thuill. = Luzula campestris

(L.) DC. in Lam. \& DC. var. multiflora (Retz.) Lej.

Juncus lampocarpus Ehrh. ex Hoffm. = Juncus articulatus L.

Juncus leersii T. Marsson = Juncus effusus L. var. conglomeratus (L.) Engelm. ex A. Gray

Juncus litorum Rydb.= Juncus balticus Willd. var. littoralis Engelm.

Juncus longicaudatus (Engelm.) Mackz. = Juncus canadensis J. Gay ex LaHarpe

Juncus macer S. Gray = Juncus tenuis Willd.

Juncus macer var. anthelatus (Wieg.) Fern. = Juncus tenuis Willd.

Juncus megacephalus (Torr.) Wood, not M. Curtis $=$ Juncus torreyi Cov.

J. marginatus Rostk., Grass-leaved Rush var. marginatus

$=\mathrm{J}$. marginatus var. paucicapitatus Engelm.

$=\mathrm{J}$. marginatus var. vulgaris Engelm.

Juncus multiflorus Ehrh. = Luzula campestris (L.)

DC. in Lam. \& DC. var. multiflora (Retz.) Lej.

J. nodosus L., Knotted Rush

$=\mathrm{J}$. paradoxus Meyer

Juncus nodosus var. megacephalus Torr. $=$ Juncus torreyi Cov.

Juncus pallescens Meyer $=$ Juncus acuminatus Michx.

Juncus paradoxus Meyer $=$ Juncus nodosus L.

Juncus paradoxus Meyer $=$ Juncus acuminatus Michx.

Juncus polycephalus var. crassifolius Torr. $=$ Juncus scirpoides Lam.
Juncus polycephalus var. tenuifolius Michx. = Juncus scirpoides Lam.

Juncus polycephalus var. depauperatus Torr. $=$ Juncus brachycephalus (Engelm.) Buch.

Juncus pondii Wood = Juncus acuminatus Michx.

J. scirpoides Lam., Scirpus-like Rush

$=\mathrm{J}$. polycephalus var. crassifolius Torr.

$=\mathrm{J}$. polycephalus var. tenuifolius Michx.

$=\mathrm{J}$. scirpoides var. genuinus Buch.

$=\mathrm{J}$. scirpoides var. meridionalis Buch.

J. subcaudatus (Engelm.) Cov. \& S. Blake, Narrow-panicled Rush, Wood's Rush $=\mathrm{J}$. canadensis var. subcaudatus Engelm.

J. tenuis Willd., Path-rush, Yard-rush $=\mathrm{J}$. bicornis Michx.

$=\mathrm{J}$. bicornis var. williamsii (Fern.) Vict.

$=\mathrm{J}$. macer S. Gray

$=$ J. macer var. anthelatus (Wieg.) Fern.

$=\mathrm{J}$. tenuis var. anthelatus Wieg.

$=\mathrm{J}$. tenuis var. williamsii Fern.

Juncus tenuis Willd. var. dudleyi (Wieg.) F. Hermann ex I. Johnston = Juncus dudleyi Wieg.

Juncus tenuis Willd. var. uniflorus (Farw.) Farw. = Juncus dudleyi Wieg.

J. torreyi Cov., Torrey's Rush

= J. megacephalus (Torr.) Wood, not M.

Curtis

$=\mathrm{J}$. nodosus var. megacephalus Torr.

JUNIPERUS L. (Cupressaceae)

Juniperus caroliniana Michx. $=$ Juniperus virginiana $\mathrm{L}$.

J. communis L., Common Juniper

var. communis

$=\mathrm{J}$. communis var. siberica (Burgsdorf)

Rydb.

var. depressa Pursh, Spreading Juniper

$=\mathrm{J}$. canadensis Burgsdorf

$=\mathrm{J}$. communis var. canadensis (Burgsdorf)

Louden

$=\mathrm{J}$. communis var. depressa Pursh

$=\mathrm{J}$. communis var. montana Ait.

$=\mathrm{J}$. communis var. saxatilis Pallas

$=\mathrm{J}$. depressa (Pursh) Raf.

$=\mathrm{J}$. sibirica Burgsdorf

Juncus depressa (Pursh) Raf. $=$ Juniperus

communis L. var. depressa Pursh 
J. horizontalis Moench, Creeping Juniper,

Prostrate Juniper, Creeping Cedar

$=\mathrm{J}$. hudsonica Forbes

$=\mathrm{J}$. prostrata Pers.

$=\mathrm{J}$. repens Nutt.

$=\mathrm{J}$. sabina Hooker var. horizontalis Hort.

$=\mathrm{J}$. sabina var. procumbens Pursh

Juniperus hudsonica Forbes $=$ Juniperus

horizontalis Moench

Juniperus prostrata Pers. $=$ Juniperus horizontalis

Moench

Juniperus repens Nutt. $=$ Juniperus horizontalis

Moench

Juniperus sabina Hooker $=$ Juniperus virginiana L.

Juniperus sabina Hooker var. horizontalis Hort. = Juniperus horizontalis Moench

Juniperus sabina var. procumbens Pursh $=$ Juniperus horizontalis Moench

Juniperus sibirica Burgsdorf = Juniperus communis L. var. depressa Pursh

J. virginiana L., Red Cedar

$=\mathrm{J}$. caroliniana Michx.

$=\mathrm{J}$. sabina Hooker

$=\mathrm{J}$. virginiana var crebra Fern. \& Grisc.

JUSTICIA L. (Acanthaceae)

J. americana (L.) Vahl., Water-willow

$=$ Dianthera americana L.

KALMIA L. (Ericaceae)

K. angustifolia L., Sheep-laurel, Lambkill, Dwarf Lawrel, Wicky, Sheepkill, Pigkill

Kalmia glauca Ait. = Kalmia polifolia Wang .

K. latifolia L., Mountain-laurel, Calico-bush, Spoonwood, Ivy-bush

K. polifolia Wang., Bog-laurel

$=$ K. glauca Ait.

KNAUTIA L. (Dipsacaceae)

*K. arvensis (L.) Coulter, Bluebuttons

$=$ Scabiosa arvensis L.

Kneiffia perennis (L.) Pennell $=$ Oenothera perennis L.

Kneiffia pratensis Small = Oenothera pilosella Raf.

Kneiffia tetragona (Roth) Pennell $=$ Oenothera fruticosa L. ssp. glauca (Michx.) Straley

Kochia scoparia (L.) Roth ex Schrad. = Bassia scoparia (L.) A. Scott
Koellia flexuosa of NY reports, not (Walt.) MacM. $=$ Pycnanthemum tenuifolium Schrad.

Koellia incana (L.) Kuntze = Pycnanthemum incanum (L.) Michx.

Koellia torrei (Benth.) Kuntze $=$ Pycnanthemum torrei Benth.

Koellia virginiana (L.) Kuntze $=$ Pycnanthemum virginianum (L.) Durieu \& Jackson ex Fern. \& B. Robinson

Konigia maritima (L.) R. Br. ex Denh. \& Clapp = Lobularia maritima (L.) Desv.

Krigia amplexicaulis (Michx.) Nutt. = Krigia biflora (Walt.) Blake

Krigia biflora (Walt. Blake (excluded by Zander \& Pierce 1979)

Lacinaria aspera (Michx.) Greene $=$ Liatris aspera Michx.

Lacinaria cylindrica (Michx.) Kuntze = Liatris cylindracea Michx.

Lacinaria pycnostachya (Michx.) Kuntze. $=$ Liatris pycnostachya Michx.

Lacinaria spicata (L.) Kuntze $=$ Liatris spicata (L.) Willd.

LACTUCA L. (Asteraceae)

L. biennis (Moench) Fern., Tall Blue Lettuce, Wild Lettuce

$=$ L. leucophaea (Willd.) A. Gray

$=$ L. spicata (of authors), not (Lam.) Kuntze

= Mulgedium leucophaeum (Willd.) DC.

L. canadensis L., Wild Lettuce.

var. canadensis

$=$ L. canadensis var. latifolia Kuntze

$=$ L. elongata Muhl. according to House, 1924 p. 668

Lactuca elongata Muhl. = Lactuca canadensis L.

Lactuca elongata var. sanguinea Torrey \& A. Gray $=$ Lactuca hirsuta Muhl. ex Nutt.

L. hirsuta Muhl. ex Nutt., Hairy Wood-lettuce, Downy Lettuce

= L. elongata var. sanguinea Torrey \& A. Gray

$=$ L. hursuta var. sanguinea (Bigel.) Fern.

Lactuca leucophaea (Willd.) A. Gray = Lactua biennis (Moench) Fern.

Lactuca pulchella (Pursh) DC. = Lactuca tatarica (L.) Meyer ssp. pulchella (Pursh) Stebbins

*L. saligna L., Willow-leaf Lettuce 
*L. sativa L., Entire-leaved Prickly Lettuce, Garden Lettuce

$=\mathrm{L}$. scariola L. in part

= L. scariola L. var. integrata Gren. \& Godr.

Lactuca scariola L., in part = Lactuca serriola L.

Lactuca scariola var. integrata Gren.\& Godr. = Lactuca sativa L.

*L. serriola L., Prickly Lettuce

$=$ L. scariola L., in part

$=\mathrm{L}$. virosa L.

Lactuca virosa L. = Lactuca serriola L.

Lactuca spicata (of authors) not (Lam.) Kuntze = Lactuca biennis (Moench) Fern.

*L. tatarica (L.) Meyer, Blue Lettuce var. pulchella (Pursh) Breitung $=$ L. pulchella (Pursh) DC.

$=$ L. tatarica ssp. pulchella (Pursh) Stebbins

LAMIUM L. (Lamiaceae)

*L. amplexicaule L., Henbit, Dead-nettle

*L. maculatum L., Spotted Dead-nettle

*L. purpureum L., Red or Purple Dead Nettle var. purpureum

Lampsana communis L. of Gray's Manual, ed 5, 1867 = Lapsana communis L.

LAPORTEA Gaud. ex Freyc. (Urticaceae)

L. canadensis (L.) Wedd., Wood-nettle, Giant

Stinging Nettle

$=$ Urtica canadensis L.

$=\mathrm{U}$. divaricata $\mathrm{L}$.

= Urticastrum divaricatum (L.) Kuntze

LAPPULA Moench (Boraginaceae)

Lappula echinata Gilib. = Lappula squarrosa

(Retz.) Dumort.

*L. squarrosa (Retz.) Dumort., European

Stickseed

= Echinospermum lappula (L.) Lehm.]

$=$ Lappula echinata Gilib.

$=$ L. lappula (L.) Karst.

Lappula virginiana (L.) Greene $=$ Hackelia

virginiana (L.) Johnst.

LAPSANA L. (Asteraceae)

*L. communis L., Nipplewort

= Lampsana communis L. of Gray's Manual, ed 5, 1867.
LARIX Mill. (Pinaceae)

Larix americana Michx. $=$ Larix laricina (DuRoi)

K. Koch

*L. decidua Mill., European Larch

L. laricina (DuRoi) K. Koch, Tamarack, American Larch

= L. americana Michx.

Lasallea ericoides (L.) Semple \& Brouillet $=$ Aster ericoides L.

Lasallea novae-angliae (L.) Semple \& Brouillet = Aster novae-angliae L.

LATHYRUS L. (Fabaceae)

L. japonicus Willd., Beach-pea

var. maritimus (L.) Kartesz \& Gandhi

$=$ L. japonicus var. glaber (Sér.) Fern.

$=\mathrm{L}$. maritimus Bigel.

$=$ L. maritimus var. glaber (Sér.) Eames

$=$ Pisum maritimum L.

*L. latifolius L., Everlasting Pea, Perennial Pea

Lathyrus maritimus Bigel. $=$ Lathyrus japonicus

WIlld. var. maritimus (L.) Kartesz \& Gandhi

Lathyrus maritimus var. glaber (Sér.) Eames = Lathyrus japonicus Willd. var. maritimus

(L.) Kartesz \& Gandhi

Lathyrus myrtifolius Muhl. ex Willd. = Lathyrus palustris L.

L. ochroleucus Hook., Cream-colored Vetchling Lathyrus pilosus Cham. $=$ Lathyrus palustris L.

L. palustris L., Vetchling, Marsh-vetch or -pea = L. palustris var. linearifolius Sér. ex DC.

$=\mathrm{L}$. palustris var. macranthus $(\mathrm{T}$. White) Fern.

$=\mathrm{L}$. palustris var. myrtifolius (Muhl. ex

Willd.) A. Gray

$=\mathrm{L}$. palustris var. pilosus (Cham.) Ledeb.

$=$ L. myrtifolius Muhl. ex Willd.

$=\mathrm{L}$. pilosus Cham.

*L. sylvestris L., Perennial or Everlasting Pea

*L. tuberosus L., Tuberous Vetchling, Earth-nut, Yellow Vetchling, Dutch Mice, Field-pea

LECHEA L. (Cistaceae)

L. intermedia Leggett ex Britt., Large-podded

Pinweed

$=\mathrm{L}$. intermedia var. laurentiana Hodgdon

$=$ L. juniperina Bickn.

$=$ L. minor Hooker in part 
Lechea juniperina Bickn. = Lechea intermedia Leggett ex Britt.

Lechea major L. $=$ Helianthemum canadense (L.) Michx.

[L. minor L., Thyme-leaved Pinweed, of doubtful occurrence (Zenkert 1934).]

Lechea minor Hooker in part $=$ Lechea intermedia Leggett ex Britt.

L. mucronata Raf., Hairy Pinweed $=$ L. stricta of NY reports, not Legg. ex Britt. $=\mathrm{L}$. villosa Ell.

Lechea stricta of NY reports, not Legg. ex Britt. = Lechea mucronata Raf.

Lechea thymifolia Michx. = Lechea minor L.

Lechea villosa Ell. = Lechea mucronata Raf.

Ledum decumbens of early NY reports, not (Ait.) Lodd. $=$ Rhododendron groenlandicum (Oeder) Kron \& Judd

Ledum groenlandicum Oeder $=$ Rhododendron groenlandicum (Oeder) Kron \& Judd

Ledum latifolium Ait. $=$ Rhododendron groenlandicum (Oeder) Kron \& Judd

Ledum palustre ssp. groenlandicum (Oeder) Hultén $=$ Rhododendron groenlandicum (Oeder) Kron \& Judd

LEERSIA Sw. (Poaceae)

L. oryzoides (L.) Sw., Rice Cut-grass

$=$ Homalocenchrus oryzoides (L.) Poll.

L. virginica Willd., White Grass

$=$ Homalocenchrus virginicus (Willd.) Britt.

$=$ Leersia virginica var. ovata (Poir.) Fern.

Leersia virginica var. ovata (Poir.) Fern. $=$ Leersia virginica Willd.

Leguminosae $=$ Fabaceae $\&$ Caesalpiniaceae

LEMNA L. (Lemnaceae)

L. minor L., Lesser Duckweed

$=$ L. obscura (Austin) Daubs

$=$ L. turionifera Landolt

Lemna obscura (Austin) Daubs = Lemna minor L.

Lemna polyrhiza L. $=$ Spirodela polyrhiza (L.) Schleid.

L. trisulca L., Star Duckweed

Lemna turionifera Landolt = Lemna minor L .

LEONTODON L. (Asteraceae)

*L. autumnalis L., Fall Dandelion, Hawkbit = Apargia autumnale (L.) Hoffm.
$=$ Leontodon autumnalis ssp. pratensis (Link) Arcang

$=$ Virea autumnalis (L.) S. Gray

$=\mathrm{V}$. autumnalis var. pratensis (L.) House

Leontodon hastilis L. = Leontodon hispidus L.

Leontodon hastilis L. var. glabratus (Koch) Bisch. $=$ Leontodon hispidus L.

Leontodon hastilis var. vulgaris Koch $=$ Leontodon hispidus L.

*L. hispidus L., Common Hawkbit

= Apargia hispida (L.) Willd.

$=$ Leontodon hastilis L.

$=$ L. hastilis L. var. glabratus (Koch) Bisch.

$=\mathrm{L}$. hastilis var. vulgaris Koch

$=$ L. hispidus ssp. danubialis (Jacq.) Simonk.

$=$ Virea hispida (L.) S. Gray

Leontodon laevigatus Willd. $=$ Taraxacum laevigatum (Wild.) DC.

Leontodon leysseri (Wallr.) Beck = Leontodon taraxacoides (Vill.) Merat

Leontodon nudicaulis of authors, not L. = Leontodon leysseri (Wallr.) G.Beck.

Leontodon taraxacum L. = Taraxacum officinale Weber ex Wiggers

*L. taraxacoides (Vill.) Merat, Hairy Hawkbit

$=$ Apargia nudicaulis (of authors)

$=$ Leontodon leysseri (Wallr.) Beck

$=\mathrm{L}$. nudicaulis of authors, not $\mathrm{L}$.

$=$ Virea nudicaulis (L.) House

Leontodon taraxacum L. = Taraxacum officinale

Weber ex Wiggers

LEONURUS L. (Lamiaceae)

*L. cardiaca L., Motherwort

$=\mathrm{L}$. glaucescens of NY reports, not Bunge ex Ledeb.

Leonurus glaucescens of NY reports, not Bunge ex Ledeb. $=$ Leonurus cardiaca L .

Lepachys pinnata (Vent.) Torr. \& A. Gray = Ratibida pinnata (Vent.) Barnh.

LEPIDIUM L. (Brassicaceae)

*L. campestre (L.) R. Br. ex Ait., Field

Peppergrass, Cow-cress

$=$ Thlaspi campestre L.

*L. densiflorum Schrad., Peppergrass, Bird's

Peppergrass, Wild Peppergrass

$=\mathrm{L}$. intermedium (of authors)

$=$ L. neglectum Thell. 
Lepidium draba L. = Cardaria draba (L.) Desv.

Lepidium intermedium (of authors) = Lepidium densiflorum Schrad.

Lepidium neglectum Thell. $=$ Lepidium densiflorum Schrad.

*L. ruderale L., Roadside Peppergrass, Stinking Peppergrass

L. virginicum L., Common Peppergrass, Poor Man's Pepper

Leptamnium virginianum (L.) Raf. = Epifagus virginiana (L.) Bart.

Leptilon canadense (L.) Britt. ex Britt. \& Brown = Conyza canadensis (L.) Cronq. var. canadensis

Leptochloa fascicularis (Lam.) A. Gray = Diplachne fascicularis (Lam.) Beauv.

Leptochloa fusca (L.) Kunth. ssp. fascicularis (Lam.) N. Snow = Diplachne fascicularis (Lam.) Beauv.

Leptoloma cognatum (Schult.) Chase $=$ Digitaria cognatum (Schult.) Pilger

LESPEDEZA Michx. (Fabaceae)

L. capitata Michx., Round-headed Bush-clover $=$ L. capitata var. velutina (Bickn.) Fern.

= L. capitata var. vulgaris Torr. \& A. Gray

Lespedeza capitata var. velutina (Bickn.) Fern. = Lespedeza capitata Michx.

Lespedeza capitata var. vulgaris Torr. \& A. Gray = Lespedeza capitata Michx.

Lespedeza fruitescens L., not Ell. or Britt. = Lespedeza violacea (L.) Pers.

L. hirta (L.) Hornem., Hairy Bush-clover

L. hirta $\times$ intermedia $=$ L. $\times$ nuttallii Darl.

L. intermedia (Wats.) Britt., Wand-like Bushclover

$=\mathrm{L}$. violacea (of local authors)

[Lespedeza stuevei Nutt. has been excluded from our flora by Zander and Pierce (1979); there exists a report unsubstantiated by specimens for Erie Co. (Young 1998).]

L. stuevii $\times$ virginica $=$ L. $\times$ neglecta Mackz. \& Bush

L. violacea (L.) Pers., Bush-clover

$=$ Hedysarum violaceum $\mathrm{L}$.

= Lespedeza frutescens L., not Ell. or Britt.

Lespedeza violacea (of local authors) $=$ Lespedeza intermedia (Wats.) Britt.
LEUCANTHEMUM Mill. (Asteraceae)

Leucanthemum parthenium Godron. $=$ Tanacetum parthenium (L.) Schultz.

*L. vulgare Lam., Ox-eye Daisy, White Daisy, Whiteweed, Marguerite, Field-daisy

$=$ Chrysanthemum leucanthemum L.

$=$ C. leucanthemum var. pinnatifidum Lecoq. \& Lamotte

LIATRIS Gaertn. \& Schreb. (Asteraceae)

*L. aspera Michx., Rough Blazing-star $=$ Lacinaria aspera (Michx.) Greene

L. cylindracea Michx., Cylindric Blazing-star $=$ Lacinaria cylindrica (Michx.) Kuntze

*L. pycnostachya Michx., Prairie Buttonsnakeroot, Gayfeather $=$ Lacinaria pycnostachya (Michx.) Kuntze.

*L. spicata (L.) Willd., Blazing-star $=$ Lacinaria spicata (L.) Kuntze

LIGUSTRUM L. (Oleaceae)

*L. obtusifolium Sieb. \& Zucc., Privet

*L. vulgare L., Privet, Hedge-privet, Prim

LILIUM L. (Liliaceae)

L. canadense L., Canada Lily, Wild Yellow Lily ssp. canadense

L. michiganense Farw., Michigan Lily

L. philadelphicum L., Wood-lily, Orange-red Lily

L. superbum L., Turk's-cap Lily

Limnorchis dilatata (Pursh) Rydb. ex Britt. = Platanthera dilatata (Pursh) Lindl. ex Beck

Limnorchis dilatata var. linearifolius Rybd. = Platanthera dilatata (Pursh) Lindl. ex Beck

Limnochoris hyperborea (L.) Rydb. ex Britt. = Platanthera hyperborea (L.) Lindl.

LINARIA Mill. (Scrophulariaceae)

L. canadensis (L.) Dumort., Old-field Toadflax

Linaria cymbalaria (L.) Mill. = Cymbalaria muralis Gaertn. Mey.\& Scherb.

*L. dalmatica (L.) Mill., Dalmatian Toadflax $=$ L. genistifolia (L.) Mill. ssp. dalmatica (L.) Maire \& Petit.

Linaria genistifolia (L.) Mill. ssp. dalmatica (L.) Maire \& Petit. = Linaria dalmatica $(\mathrm{L}$. Mill.

Linaria linaria (L.) Karst. = Linaria vulgaris Mill. 
Linaria minor (L.) Desf. $=$ Chaenorrhinum minus

(L.) Lange

*L. vulgaris Mill., Butter-and-Eggs

$=$ L. linaria (L.) Karst.

LINDERA Thunb. (Lauraceae)

L. benzoin (L.) Blume, Spicebush, Benzoin-bush, Benjamin-bush

$=$ Benzoin aestivale (L.) Nees

$=$ B. odoriferum Nees \& ed 2 of Gray's Manual

LINDERNIA All. (Scrophulariaceae)

L. dubia (L.) Pennell, False Pimpernel var. dubia

$=$ Ilysanthes dubia (L.) Barnh.

= Ilysanthes dubia var. riparia (Raf.) Fern.

$=$ Ilysanthes dubia var. inundata (Pennell) Pennell

$=$ Ilysanthes gratioloides (L.) Benth. in DC.

$=$ Lindernia dubia var. riparia (Raf.) Fern.

LINNAEA L. (Caprifoliaceae)

L. borealis L., Twin-flower ssp. longiflora (Torr.) Hultén

$=\mathrm{L}$. borealis ssp. americana (Forbes) Hultén

$=\mathrm{L}$. borealis L. var. americana (Forbes) Rehd.

$=\mathrm{L}$. borealis var. longiflora Torr.

LINUM L. (Linaceae)

Linum humile Mill. $=$ Linum usitatissimum L.

L. medium (Planch.) Britt., Yellow Flax, Wild Flax

var. medium

var. texanum (Planch.) Fern., Southern Yellow Flax

L. striatum Walt., Stiff Yellow Flax

$=$ L. striatum var. multijugum Fern.

L. sulcatum Riddell, Grooved Yellow Flax, Yellow Wild Flax

*L. usitatissimum L., Common Flax

$=\mathrm{L}$. humile Mill.

$=$ L. usitatissimum var. humile (Mill.) Pers.

L. virginianum L., Slender Yellow Flax

LIPARIS L. Rich. (Orchidaceae)

L. liliifolia (L.) Rich. ex Ker, Lily-leaved Twayblade, Large or Purple Twayblade
$=$ L. liliifolia of authors

L. loeselii (L.) L. Rich., Loesel's Twayblade, Bogtwayblade, Yellow Twayblade, Fen-orchid

Liquidambar peregrina L. $=$ Comptonia peregrina (L.) Coult.

LIRIODENDRON L. (Magnoliaceae)

L. tulipifera L., Tulip Tree, Tulip Poplar, Yellow Poplar

LISTERA R. Br. (Orchidaceae)

L. australis Lindl., Southern Twayblade $=$ Ophrys auriculata (Wieg.) House.

L. convallarioides (Sw.) Nutt. ex Ell., Broadlipped Twayblade

L. cordata (L.) R. Br., Heartleaf Twayblade

LITHOSPERMUM L. (Boraginaceae)

Lithospermum arvense L. = Buglossoides arvense (L.) Johnst.

L. caroliniense (Walt. ex Gmel.) MacMill., Hairy Puccoon, Golden Puccoon

var. croceum (Fern.) Cronq.

$=$ L. caroliniense ssp. croceum (Fern.) Cusick

$=$ L. croceum Fern.

$=$ L. gmelinii (of authors)

$=\mathrm{L}$. hirtum (of authors)

Lithospermum croceum Fern. = Lithospermum caroliniense (Walt. ex Gmel.) MacMill. var. croceum (Fern.) Cronq.

Lithospermum gmelinii (of authors) $=$ Lithospermum caroliniense (Walt. ex Gmel.) MacMill. var. croceum (Fern.) Cronq.

Lithospermum hirtum (of authors) $=$ Lithospermum caroliniense (Walt. ex Gmel.) MacMill. var. croceum (Fern.) Cronq.

L. latifolium Michx., Broad-leaved Gromwell, Puccoon

*L. officinale L., Common Gromwell, European Gromwell

LITTORELLA Berg. (Plantaginaceae)

L. uniflora (L.) Aschers., Shore-grass, Littorella $=$ L. americana Fern.

= L. uniflora var. americana (Fern.) Gleason

LOBELIA L. (Lobeliaceae) 
L. cardinalis L., Cardinal-flower, Indian Pink

L. inflata L., Indian-tobacco Lobelia

L. kalmii L., Kalm's Lobelia, Brook-lobelia

L. siphilitica L., Great Lobelia, Blue Cardinalflower

L. spicata Lam., Pale-Spiked Lobelia

LOBULARIA Desv. (Brassicaceae)

*L. maritima (L.) Desv., Sweet or Seaside Alyssum

= Clypeola maritima L.

= Koniga maritima (L.) R. Br. ex Denh. \& Clapp

LOLIUM L. (Poaceae)

Lolium multiflorum Lam. = Lolium perenne L. var. aristatum Willd.

*L. perenne L., English or Perennial Ryegrass

*var. aristatum Willd., Italian Ryegrass

$=$ L. multiflorum Lam.

$=$ L. multiflorum var. diminutum Mutel

$=$ L. perenne ssp. multiflorum (Lam.) Husnot

$=\mathrm{L}$. perenne var. multiflorum (Lam.) Thuill. ex Bast.

*var. perenne

*L. pratense (Hudson) S. Darbyshire, Meadowfescue, English Bluegrass

$=$ Festuca elatior L., a nomen confusum

$=$ F. pratensis Hudson

$=$ Schedonorus pratensis (Huds.) P. Beauv.

*L. temulentum L., Darnel, Poisongrass

LONICERA L. (Caprifoliaceae)

Lonicera bella Zabel $=[$ Lonicera morrowii $\times$ tatarica $=$ L.$\times$ bella Zabel $]$

Lonicera caerulea (of American authors) $=$ Lonicera villosa (Michx.) R.\& S.

L. canadensis Bartr., Fly-honeysuckle $=$ L. ciliata Muhl.

Lonicera ciliata Muhl. $=$ Lonicera canadensis Bartr.

L. dioica L., Smooth-leaved Honeysuckle, Wild or Mountain-honeysuckle

var. dioica

= L. parviflora Lam.

var. glaucescens (Rydb.) Butters, Glaucous

Honeysuckle

$=\mathrm{L}$. glaucescens Rydb.

$=$ L. parviflora var. douglassii A. Gray
Lonicera glaucescens Rydb. = Lonicera dioica var glaucescens (Rydb.) Butters

L. hirsuta Eat., Hairy Honeysuckle $=\mathrm{L}$. hirsuta var. interior Gleason

*L. japonica Thunb., Japanese Honeysuckle, Gold-and-Silver-flower

*L. maackii (Rupr.) Maxim., Honeysuckle

*L. morrowii A. Gray, Morrow's Honeysuckle

$*$ L. morrowii $\times$ tatarica $=$ L. $\times$ bella Zabel, Flyhoneysuckle

$=\mathrm{L}$. bella Zabel

L. oblongifolia (Goldie) Hook., Swamp Flyhoneysuckle

$=$ L. oblongifolia var. altissima (Jennings) Rehd.

Lonicera parviflora Lam. $=$ Lonicera dioica var. dioica L.

Lonicera parviflora var. douglassii A. Gray = Lonicera dioica var. glaucescens (Rydb.) Butters

Lonicera sempervirens L. (excluded by Zander \& Pierce 1979)

*L. tatarica L., Tartarian Honeysuckle

L. villosa (Michx.) R.\& S., Mountain Flyhoneysuckle

$=\mathrm{L}$. caerulea (of American authors)

$=\mathrm{L}$. villosa var. tonsa Fern.

*L. xylosteum L., European Fly-honeysuckle

Lophanthus nepetoides (L.) Benth. = Agastache nepetoides (L.) Kuntze

Lophanthus scrophulariaefolius (Willd.) Benth. = Agastache scrophulariaefolia (Willd.) Kuntze

LOTUS L. (Fabaceae)

*L. corniculata L., Bird-foot Trefoil, Eggs \& Bacon

$=$ Lotus corniculatus of authors

LUDWIGIA L. (Onagraceae)

L. palustris (L.) Ell., Water-purslane var. americana (L.) Ell.

= L. palustris var. americana (DC.) Fern. \& Griscom

L. polycarpa Short \& Peter, Many-fruited False Loosestrife

LUNARIA L. (Brassicaceae)

*L. annua L., Honesty, Money-plant, Satin-flower 
$=\mathrm{L}$. biennis Moench

LUPINUS L. (Fabaceae)

L. perennis L., Wild Lupine, Sundial-lupine $=\mathrm{L}$. perennis var. occidentalis $\mathrm{S}$. Wats

LUZULA DC. (Juncaceae)

L. acuminata Raf., Hairy Woodrush

$=$ Juncoides carolinae (S. Wats.) Kuntze

$=$ Luzula acuminata var. carolinae (S. Wats.) Fern.

$=$ L. acuminata var. saltuensis (Fern.) Fern.

$=\mathrm{L}$. pilosa (of authors)

$=$ L. pilosa var. americana Schultes \&

Schultes

$=$ L. saltuensis Fern.

L. campestris (L.) DC. in Lam. \& DC., Common or Field-woodrush

var. echinata (Small) Fern. \& Wieg.

$=$ L. echinata (Small) F. Hermann

var. multiflora (Retz.) Lej.

$=$ Juncoides intermedium (Thuill.) Rydb.

$=$ Juncus intermedius Thuill.

$=\mathrm{J}$. multiflorus Ehrh.

$=$ Luzula campestris ssp. multiflora (Retz.) Buch.

$=$ L. erecta Desv.

$=\mathrm{L}$. intermedia var. multiflora Spenner

$=$ L. multiflora (Retz.) Lej.

$=$ L. multiflora var. frigida Buch.

var. pallescens (Wahl.) Wahl., Common Woodrush

Luzula echinata $($ Small $)$ F. Hermann = Luzula campestris var. echinata (Small) Fern. \& Wieg.

Luzula erecta Desv. $=$ Luzula campestris (L.) DC. in Lam. \& DC. var. multiflora (Retz.) Lej.

Luzula intermedia var. multiflora Spenner $=$ Luzula campestris (L.) DC. in Lam. \& DC. var. multiflora (Retz.) Lej.

Luzula multiflora (Retz.) Lej. = Luzula campestris

(L.) DC. in Lam. \& DC. var. multiflora (Retz.) Lej.

Luzula multiflora var. frigida Buch. $=$ Luzula campestris (L.) DC. in Lam. \& DC. var. multiflora (Retz.) Lej.

Luzula pilosa (of authors) = Luzula acuminata Raf.

Luzula pilosa var. americana Schultes \& Schultes $=$ Luzula acuminata Raf.
Luzula saltuensis Fern. = Luzula acuminata Raf.

LYCHNIS L. (Caryophyllaceae)

Lychnis alba Mill. = Silene latifolia Poir.

Lychnis arvensis Gaertn., Meyer \& Scherb. = Silene latifolia Poir.

*L. coronaria (L.) Desr. Mullein-pink, Rosecampion

= Agrostemma coronaria L.

Lychnis githago (L.) Scop. = Agrostemma githago L.

Lychnis vespertina Sibth. $=$ Silene latifolia Poir

LYCIUM L. (Solanaceae)

*L. barbarum L., Matrimony-vine

$=\mathrm{L}$. chinense Mill.

$=$ L. halamifolium Mill.

$=\mathrm{L}$. vulgare (Ait) Dunal in DC.

Lycium chinense Mill. = Lycium barbarum L.

Lycium halimifolium Mill. = Lycium barbarum L .

Lycium vulgare (Ait) Dunal in DC. $=$ Lycium barbarum L.

LYCOPERSICON Mill. (Solanaceae)

*L. esculentum Mill., Garden Tomato

= L. lycopersicum (L.) Karst. ex Farw.

= Solanum lycopersicum L.

Lycopersicon lycopersicum (L.) Karst. ex Farw. = Lycopersicon esculentum Mill.

LYCOPODIELLA Holub (Lycopodiaceae)

L. inundata (L.) Holub, Bog Club-moss, Marsh

Club-moss

$=$ Lycopodium inundatum $\mathrm{L}$.

LYCOPODIUM L. (Lycopodiaceae)

L. annotinum L., Stiff Club-moss, Bristly Clubmoss

var. annotinum

var. pungens (La Pyl.) Desv.

$=$ L. annotinum var. acrifolium Fern.

$=$ L. dubium Zoega

$=$ Spinulum annotinum (L.) A. Haines

$=$ S. canadense (Ness.) A. Haines

Lycopodium apodum L. = Selaginella apodum L.

L. clavatum L., Running-pine

Lycopodium complanatum L. var. complanatum of early authors = Lycopodium digitatum Dill. ex A. Br. 
Lycopodium complanatum var. habereri (House)

Boiv. $=[$ Lycopodium digitatum $\times$ tristachyum $=$ L. $\times$ habereri House $]$

Lycopodium complanatum L. var. flabelliforme Fern. = Lycopodium digitatum A. Br.

L. dendroideum Michx., Northern Tree Clubmoss, Ground-pine

$=$ Dendrolycopodium dendroideum (Michx.)

A. Haines

= Lycopodium obscurum var. dendroideum (Michx.) D. Eaton

L. digitatum Dill. ex A. Br., Ground-cedar, Southern Running-pine, Crowfoot Clubmoss

$=$ Diphasiastrum digitatum (A. Br.) Holub

= Lycopodium complanatum L. var. complanatum of early authors

$=$ L. complanatum L. var. flabelliforme Fern.

$=$ L. flabelliforme (Fern.) Blanch.

L. digitatum $\times$ tristachyum $=$ L. $\times$ habereri House

$=$ Diphasiastrum digitatum $\times$ D. tristachyum $=\mathrm{D}$. habereri (House) Holub

= Lycopodium complanatum var. habereri

(House) Boiv.

Lycopodium dubium Zoega = Lycopodium annotinum L. var. pungens (La Pyl.) Desv. Lycopodium flabelliforme (Fern.) Blanch. = Lycopodium digitatum Dill. ex A. Br.

Lycopodium lucidulum Michx. $=$ Huperzia lucidula (Michx.) Trev.

Lycopodium lucidulum var. occidentale (Clute) Wilson $=$ Huperzia lucidula (Michx.) Trev.

Lycopodium lucidulum var. tryonii Mohlenbrock = Huperzia lucidula (Michx.) Trev.

L. obscurum L., Ground-pine, Eastern Tree Clubmoss

$=$ Dendrolycopodium obscurum (L.) A. Haines

Lycopodium obscurum var. dendroideum (Michx.)

D. Eaton = Lycopodium dendroideum Michx.

Lycopodium rupestre L. $=$ Selaginella rupestris (L.) Spring

L. tristachyum Pursh, Three-spiked Club-moss, Ground Cedar, Northern Ground-pine $=$ Diphasiastrum tristachyum (Pursh) Holub $=$ Diphasium tristachyum (Pursh) Rothm.

Lycopsis arvensis L. = Anchusa arvensis (L.) Bieb.
LYCOPUS L. (Lamiaceae)

L. americanus Muhl. ex Bart., Cut-leaved Waterhorehound

$=\mathrm{L}$. americanus var. longii Benner

L. asper Greene, Western Water-horehound = L. lucidus ssp. americanus (Gray) Hultén

$=\mathrm{L}$. lucidus var. americanus Gray

*L. europaeus L., European Water-horehound $=$ L. europaeus var. mollis (Kern.) Briq.

Lycopus lucidus var. americanus Gray = Lycopus asper Greene

Lycopus membranaceus Bickn. ex Britt. = Lycopus uniflorus Michx.

L. rubellus Moench, Gypsy-wort, Waterhorehound

L. uniflorus Michx., Northern Bugleweed, Waterhorehound

$=\mathrm{L}$. membranaceus Bickn. ex Britt.

L. virginicus L., Virginia Bugleweed, Waterhorehound

LYONIA Nutt. (Ericaceae)

L. ligustrina (L.) DC., Maleberry, HeHuckleberry, Male Blueberry

Lysias hookeri (Torr. ex A. Gray) Rydb. ex Britt. = Platanthera hookeri (Torr. ex A. Gray) Lindl.

Lysias macrophylla (Goldie) House $=$ Platanthera macrophylla (Goldie) P. M. Brown

Lysias orbiculata (Pursh) Rydb. ex Britt. = Platanthera orbiculata (Pursh) Lindl.

LYSIMACHIA L. (Primulaceae)

L. ciliata L., Fringed Loosestrife $=$ Steironema ciliatum (L.) Raf.

[L. hybrida Michx., Intermediate Loosestrife] $=\mathrm{L}$. lanceolata of NY reports, not Walt.

= L. lanceolata var. hybrida (Michx.) A. Gray

$=$ Steironema hybridum (Michx.) Raf.

The species was excluded from the Niagara Frontier region by Zander and Pierce (1979). A pre-1980 specimen exists for Allegany Co. (NYFA 1990), perhaps just outside of our range. The species does occur in Ontario (Morton \& Venn 1990).]

Lysimachia lanceolata Walt. (excluded), see Lysimachia hybrida 
Lysimachia longifolia Pursh = Lysimachia quadriflora Sims

*L. nummularia L., Moneywort, Creeping Jenny, Creeping Charlie

Lysimachia producta (A. Gray) Fern. = [Lysimachia quadrifolia $\times$ terrestris $=\mathrm{L} . \times$ producta (A. Gray) Fern.]

*L. punctata L., Spotted Loosestrife, Garden Loosestrife

L. quadriflora Sims, Linear-leaved Loosestrife, Four-flowered Loosestrife

$=\mathrm{L}$. longifolia Pursh

$=$ Steironema quadriflorum (Sims) Hitchc.

L. quadrifolia $\times$ terrestris $=\mathbf{L} . \times$ producta $(A$. Gray) Fern., Elongate Loosestrife

$=$ L. producta (A. Gray) Fern.

$=$ L. stricta Aiton var. producta A. Gray

L. quadrifolia L., Whorled Loosestrife

Lysimachia stricta Ait. = Lysimachia terrestris (L.) BSP

Lysimachia stricta Aiton var. producta A. Gray = [Lysimachia quadrifolia $\times$ terrestris $=$ L. $\times$ producta (A. Gray) Fern.]

L. terrestris (L.) BSP., Bulb-bearing Loosestrife $=$ L. stricta Ait.

L. thyrsiflora L., Tufted Loosestrife $=$ Naumbergia thyrsiflora (L.) Duby

*L. vulgaris L., Garden Loosestrife

LYTHRUM L. (Lythraceae)

Lythrum petiolatum L. = Cuphea viscosissima Jacq.

L. alatum Pursh, Wing-angled Loosestrife, Winged Loosestrife

*L. salicaria L., Purple or Spiked Loosestrife Lythrum verticillatum L. $=$ Decodon verticillatus (L.) Ell.

MACLURA Nutt. (Moraceae)

Maclura aurantiaca Nutt. = Maclura pomifera (Raf. ex Sarg.) Schneid.

*M. pomifera (Raf. ex Sarg.) Schneid., Osage Orange $=$ M. aurantiaca Nutt.

$=$ Toxylon pomiferum Raf. ex Sarg.

MAGNOLIA L. (Magnoliaceae)

M. acuminata (L.) L., Cucumber-tree, Cucumbermagnolia
MAHONIA Nutt. (Berberidaceae)

*M. aquifolium (Pursh) Nutt., Oregon Grape,

Grape-holly

$=$ Berberis aquilifolium Pursh.

MAIANTHEMUM Wiggers (Liliaceae)

M. canadense Desf., False Lily-of-the-valley, Two-leaf Solomon's Seal, Wild Lily-ofthe-valley

$=$ M. canadense var. interior Fern.

$=$ Smilacina bifolia Torr.

$=$ Unifolium canadense (Desf.) Greene

$=\mathrm{U}$. canadense var. interior (Fern.) House

M. racemosum L., False Solomon's-seal, False Spikenard

$=$ Smilacina racemosa (L.) Desf.

$=\mathrm{S}$. racemosa var. cylindrata Fern.

$=$ Vagnera racemosa (L.) Morong

M. stellatum L., Star-flowered False Solomon'sseal

$=$ Smilacina stellata (L.) Desf.

= Vagnera stellata (L.) Morong

M. trifolium L., Three-leaved False Solomon'sseal

$=$ Smilacina trifolia $($ L.) Desf.

= Vagnera trifolia (L.) Morong

Malachium aquaticum (L.) Fries $=$ Myosoton aquaticum (L.) Moench

MALAXIS Solander ex Sw. (Orchidaceae)

Malaxis bayardii Fern. = Malaxis unifolia Michx.

Malaxis brachypoda (A. Gray) Fern. = Malaxis monophylla (L.) Sw. var. brachypoda (A. Gray) Morris \& Eames

M. monophyllos (L.) Sw., White Adder's-mouth $=$ Microstylis monophyllos (L.) Lindl.

var. brachypoda (A. Gray) Morris \& Eames $=$ M. brachypoda (A. Gray) Fern.

M. unifolia Michx., Green Adder's-mouth $=$ M. bayardii Fern.

$=$ Microstylis unifolia (Michx.) BSP

MALUS P. Mill. (Rosaceae)

Malus bracteata Redh. $=$ Malus coronaria (L.) Mill.

M. coronaria (L.) Mill., Wild Crabapple, American Crab, Sweet-crab

$=\mathrm{M}$. bracteata Redh.

$=$ M. coronaria var. dasycalyx Rehd. 
$=\mathrm{M}$. coronaria var. elongata Rehd. of

Zenkert, 1934

$=$ M. fragrans Rehd.

$=$ M. lancifolia Rehd.

$=$ Pyrus coronaria L.

$=$ P. coronaria var. dasycalyx (Rehd.) Fern.

$=\mathrm{P}$. coronaria var. elongata (Rehd.) Bailey

$=$ P. coronaria var. lancifolia (Rehd.) Fern.

Malus domestica Borkh. = Malus pumila Mill.

Malus fragrans Rehd. = Malus coronaria (L.) Mill.

M. glaucescens Rehd., American Crab, Wild Crab-apple

$=$ M. coronaria var. glaucescens Rehd. of Zenkert, 1934

$=$ Pyrus coronaria var. glaucescens (Rehd.) Bailey

Malus lancifolia Rehd. $=$ Malus coronaria (L.) Mill.

Malus malus (L.) Shafer $=$ Malus pumila Mill.

*M. pumila Mill., Domestic Apple

$=$ M. domestica Borkh.

$=$ M. malus (L.) Shafer

= M. sylvestris of authors, not (L.) Mill.

$=$ Pyrus malus of authors, not L.

Malus sylvestris of authors, not (L.) Mill. = Malus pumila Mill.

MALVA L. (Malvaceae)

*M. alcea L., Vervain-mallow

Malva crispa (L.) L. = Malva verticillata L.

*M. moschata L., Musk-mallow, Musk

Cheeseweed

*M. neglecta Wallr., Cheeses

$=$ M. rotundifolia (of Zenkert, 1934) of authors, not L.

Malva rotundifolia (of Zenkert, 1934), of authors, not L. = Malva neglecta Wallr.

*M. sylvestris L., High Mallow, Cheeses

*M. verticillata L.), Whorled Mallow $=$ M. crispa $(\mathrm{L}.) \mathrm{L}$.

$=\mathrm{M}$. verticillata var. crispa $\mathrm{L}$.

Malva verticillata var. crispa L. = Malva verticillata $\mathrm{L}$.

Mariana mariana (L.) Hill = Silybum marianum (L.) Gaertn.

Mariscus mariscoides (Muhl.) Kuntze $=$ Cladium mariscoides (Muhl.) Torr.

MARRUBIUM L. (Lamiaceae)
*M. vulgare L., Horehound

MARSILIA L. (Marsileaceae)

*M. quadrifolia L., Water-clover, Pepperwort

$=$ M. mexicana of NY reports, not Schlect. \& Cham.

Martynia louisiana Mill. $=$ Proboscidea louisiana (Mill.) Woot.\& Standl.

Martynia proboscidea Glox. $=$ Proboscidea louisiana (Mill.) Woot. \& Standl.

Maruta cotula (L.) DC. $=$ Anthemis cotula L.

MATRICARIA L. (Asteraceae)

*M. discoidea DC., Pineapple-weed $=$ Chamomila suaveolens (Pursh) Rydb. $=$ Matricaria matricarioides (Less.) Porter $=$ M. suaveolens (Pursh) Buch.

Matricaria inodora L. (nom. illeg.) = Matricaria perforata Merat

Matricaria maritima of NY reports, not L. = Matricaria perforata Merat

Matricaria maritima var. agrestis (Knaf) Wilm. = Matricaria perforata Merat

Matricaria maritima var. agrestis (Knaf) Wilm. = Matricaria perforata Merat

Matricaria matricarioides (Less.) Porter $=$ Matricaria discoidea DC.

Matricaria parthenium L. according to House 1924 p. $735=$ Tanacetum parthenium (L.) Schultz

*M. perforata Merat, Scentless Chamomile, Seaside-chrysanthemum

$=$ Chamomilla maritima of NY reports, not (L.) Rydb.

$=$ C. inodora (L.) Rydb.

= Matricaria inodora L. (nom. illeg.)

$=\mathrm{M}$. maritima of NY reports, not $\mathrm{L}$.

$=\mathrm{M}$. maritima var. agrestis (Knaf) Wilm.

$=$ Tripleurospermum maritimum ssp. inodorum (L.) Hyl. ex Vaar $=\mathrm{T}$. perforatum (Merat) M. Lainz

*M. recutita L., Wild Chamomile, False Sweet Chamomile, German Mayweed $=$ Chamomilla chamomilla (L.) Rydb.

$=$ C. recutita (L.) Rausch

= Matricaria chamomilla L.

Matricaria suaveolens $($ Pursh $)$ Buch. $=$ Matricaria discoidea DC. 
MATTEUCCIA Todaro (Dryopteridaceae)

Matteuccia pensylvanica (Willd.) Raym. = Matteuccia struthiopteris (L.) Todaro

M. struthiopteris (L.) Todaro, Ostrich-fern $=$ Matteuccia pensylvanica (Willd.) Raym. $=$ M. struthiopteris var. pensylvanica (Willd.) C. V.Morton = Osmunda struthiopteris L.

$=$ Pteretis nodulosa (Michx.) Nieuwl. $=$ Pteretis pensylvanica (Willd.) Fern. $=$ Struthiopteris germanica Willd.

MEDEOLA L. (Liliaceae)

M. virginiana L., Indian Cucumber-root

MEDICAGO L. (Fabaceae)

*M. lupulina L., Black Medick, Nonsuch $=\mathrm{M}$. lupulina var. glandulosa Neilr. Medicago lupulina var. glandulosa Neilr. = Medicago lupulina L.

*M. sativa L., Alfalfa, Lucerne

*ssp. sativa (ESCAPE)

MEGALODONTA Greene (Asteraceae)

M. beckii (Torr. ex Spreng.) Greene, Watermarigold

$=$ Bidens beckii Torr. ex Spreng.

Meibomia dillenii (Darl.) Kuntze in part = Desmodium perplexum Schub.

MELAMPYRUM L. (Scrophulariaceae)

Melampyrum americanum Michx. = Melampyrum lineare Desr.

M. lineare Desr., Cow-wheat

$=\mathrm{M}$. americanum Michx.

= M. lineare var. americanum (Michx.)

Beauv.

$=$ M. lineare var. latifolium Bart., not (Muhl.) Beauv.

$=\mathrm{M}$. lineare var. pectinatum (Pennell) Fern.

Melandrium noctiflorum $($ L.) Fries $=$ Silene noctiflora $\mathrm{L}$.

Melica mutica Walt, Siberian Melic, excluded by Zander \& Pierce 1979; "M. mutica as to [Ontario] reports, not of Walter" = M. altissima L., occasional garden escape, not persisting" (Morton \& Venn 1990). The species is reported by Mitchell and Tucker
(1997) in New York State, also in nonpersisting populations.

Melica purpurascens (Torr.) Hitchc. $=$ Schizachne purpurascens (Torr.) Swallen

MELILOTUS Mill. (Fabaceae)

*M. alba Desr. ex Lam., White Sweet-clover $=$ M. alba Medik., a nomen nudum

*M. altissima Thuill., Tall Yellow Sweet-clover

*M. officinalis (L.) Pallas, Yellow Sweet-clover $=$ Trifolium meliloti-officinalis of authors, not L.

$=\mathrm{T}$. officinalis $\mathrm{L}$.

MELISSA L. (Lamiaceae)

*M. officinalis L., Lemon-balm

MENISPERMUM L. (Menispermaceae)

M. canadense L., Moonseed, Yellow Parilla

MENTHA L. (Lamiaceae)

Mentha $\times$ piperita var. crispa $($ L. $)$ Koch $=$ Mentha spicata L.

Mentha aquatica L. var. crispa (L.) Benth. = Mentha spicata L.

$*$ M. aquatica $\times$ spicata $=$ M. $\times$ piperita L., Peppermint, Bergamot Mint

$=$ M. piperita L. of Zander \& Pierce (1979)

$=$ M. citrata Ehrh.

$=$ M. $\times$ piperita var. citrata (Ehrh.) Briq.

*M. arvensis L., American Wild Mint, Field-mint $=\mathrm{M}$. gentilis L.

$*$ M. arvensis $\times$ spicata $=$ M. $\times$ gracilis Sole, Red

Mint, Scotch Mint, Spotted Mint, Creeping

Whorled Mint

$=$ M. cardiaca Gerard ex Baker

$=\mathrm{M} . \times$ gentilis L. $=$ M. arvensis ssp. arvensis

$\times$ M. spicata

$=\mathrm{M} . \times$ cardiaca $($ Gray $)$ Baker

$=$ M. $\times$ gracilis Sole

M. arvensis var. canadensis (L.) Briq. = Mentha canadensis $\mathrm{L}$.

Mentha arvensis ssp. haplocalyx Briq. = Mentha canadensis L.

Mentha arvensis var. glabrata (Benth.) Fern. = Mentha canadensis L.

M. arvensis var. villosa (Benth.) S.R. Stewart = Mentha canadensis L.

M. canadensis L., Field-mint 
$=$ M. arvensis sensu auct. non L.

$=\mathrm{M}$. arvensis ssp. haplocalyx Briq.

$=$ M. arvensis var. canadensis (L.) Briq.

$=\mathrm{M}$. arvensis var. glabrata (Benth.) Fern.

$=$ M. arvensis var. villosa (Benth.) Stewart

Mentha cardiaca Gerarde ex Baker $=[$ Mentha arvensis $\times$ spicata $=$ M. $\times$ gentilis L.]

Mentha citrata Ehrh. $=[$ Mentha aquatica $\times$ spicata $=\mathrm{M} . \times$ piperita L.]

Mentha crispa L. = Mentha spicata L.

Mentha gentilis L. $=$ Mentha arvensis L.

Mentha $\times$ gentilis L. $=$ Mentha arvensis $\times$ M. spicata

Mentha $\times$ piperita L. $=$ Mentha aquatica $\times$ M. spicata

Mentha $\times$ piperita var. citrata (Ehrh.) Briq. $=$ $[$ Mentha aquatica $\times$ spicata $=$ M. $\times$ piperita L.]

$*$ M. longifolia $\times$ suaveolens $=$ M. $\times$ rotundifolia (L.) Huds., Apple-aint, Pineapple-mint $=$ M. rotundifolia (L.) Huds.

M. rotundifolia (L.) Huds. $=[$ Mentha longifolia $\times$ suaveolens $=$ M. $\times$ rotundifolia $($ L.) Huds.]

*M. spicata L., Spearmint

$=$ M. aquatica var. crispa (L.) Benth.

$=\mathrm{M}$. crispa $\mathrm{L}$.

$=$ M. $\times$ piperita var. crispa (L.) Koch

$=\mathrm{M}$. viridis $\mathrm{L}$.

Mentha viridis L. = Mentha spicata L.

MENYANTHES L. (Menyanthaceae)

M. trifoliata L. Buckbean var. minor Raf.

MERTENSIA Roth (Boraginaceae)

M. virginica (L.) Pers. ex Link, Bluebells, Virginia Bluebells, Virginia Cowslip, Roanoke-bells

Mespilus arbutifolia L. = Aronia arbutifolia (L.) Pers.

Mespilus arbutifolia var. melanocarpa Michx. = Aronia melanocarpa (Michx.) Ell.

Mespilus prunifolia Marsh. $=[$ Aronia arbutifolia $\times$ melanocarpa $=$ A. $\times$ prunifolia (Marsh.) Rehd.]

Micrampelis lobata (Michx.) Greene $=$ Echinocystis lobata (Michx.) Torr. \& A. Gray
Micromeria glabella of ON reports, not of Benth. = Calamintha arkansana (Nutt.) Shinners

Microstyis unifolia (Michx.) BSP = Malaxis unifolia Michx.

Microstylis monophyllos (L.) Lindl. = Malaxis monophylla (L.) Sw.

MIKANIA Willd. (Asteraceae)

M. scandens (L.) Willd., Climbing Boneset

MILIUM L. (Poaceae)

M. effusum L., Tall Millet-grass

MIMULUS L. (Scrophulariaceae)

M. alatus Ait., Sharp-winged Monkey-flower

*M. moschatus Dougl. ex Lindl., Musk-flower

M. ringens L., Monkey-flower, Purple or Allegheny Monkeyflower

MIRABILIS L. (Nyctaginaceae)

*M. nyctaginea (Michx.) MacM., Wild FourO'clock

$=$ Allionia nyctaginea Michx.

= Oxybaphus nyctagineus (Michx.) Sweet

MISCANTHUS Anderss. (Poaceae)

*M. sinensis Anderss., Eulalia

MITCHELLA L. (Rubiaceae)

M. repens L., Partridge-berry, Twin-berry, Running-box, Two-Eyed Berry, Mitchella

MITELLA L. (Saxifragaceae)

M. diphylla L., Miterwort

= M. oppositifolia Rydb.

M. nuda L., Naked Miterwort

$=\mathrm{M}$. reniformis Lam.

Mitella oppositifolia Rydb. = Mitella diphylla L.

Mitella reniformis Lam. $=$ Mitella nuda L.

MOEHRINGIA L. (Caryophyllaceae)

M. lateriflora (L.) Fenzl, Side-flowering

Sandwort, Grove-sandwort, Blunt-leaf

Sandwort

$=$ Arenaria angustifolia Regel

$=$ A. lateriflora $\mathrm{L}$.

$=$ Stellaria biflora Pursh

MOLLUGO L. (Molluginaceae) 
*M. verticillata L., Carpet-weed, Indian

Chickweed, Devil's Grip

$=\mathrm{M}$. berteriana Sér.

$=$ M. cerviana of NY reports, not (L.) Sér.

MONARDA L. (Lamiaceae)

M. clinopodia L., Basil-balm

$=$ M. fistulosa L. var. clinopodia (L.) Cooperrider

M. didyma L., Oswego Tea, Bee-balm

M. fistulosa L. Wild Bergamot, Horsemint $=$ M. fistulosa var. mollis (L.) Benth. $=$ M. mollis L.

Monarda fistulosa L. var. clinopodia (L.) Cooperrider $=$ Monarda clinopodia L .

Monarda fistulosa var. rubra A. Gray = Monarda media Willd.

M. media Willd., Purple Bergamot, Bee-balm $=\mathrm{M} . \times$ media of authors, not Willd. $=\mathrm{M}$. fistulosa var. rubra A. Gray

= M. media Willd. of Zander \& Pierce, 1979

Monarda $\times$ media of authors, not Willd. $=$ Monarda media Willd.

Monarda mollis L. = Monarda fistulosa L.

MONESES Salisb. ex S. Gray (Ericaceae)

M. uniflora (L.) A. Gray, One-flowered Shinleaf, One-flowered Pyrola

$=$ Pyrola uniflora $\mathrm{L}$.

MONOTROPA L. (Ericaceae)

Monotropa brittonii Small = Monotropa uniflora L.

M. hypopithys L., Pinesap

= Hypopithys americana (DC.) Small

$=$ H. lanuginosa (Michx.) Raf., not Nutt.

$=\mathrm{H}$. multiflora var. americana DC.

= Monotropa hypopithys Michx., not L.

$=\mathrm{M}$. lanuginosa Michx.

Monotropa lanuginosa Michx. = Monotropa hypopithys L.

Monotropa procera Torr. ex Eaton $=$ Pterospora andromedea Nutt.

M. uniflora L., Indian Pipe

$=$ M. brittonii Small

Montelia tamariscina (Nutt.) A. Gray = Amaranthus tuberculatus (Moq. ex DC.)

Sauer

Morella caroliniana Small $=$ Myrica pensylvanica Loisel. ex Duhamel
MORUS L. (Moraceae)

*M. alba L., White Mulberry

= M. alba var. tatarica (Pallas) Sér.

$=\mathrm{M}$. nigra of NY reports, not L.

$=$ M. tatarica Pallas, not L.

Morus nigra of NY reports, not L. = Morus alba L.

M. rubra L., Red Mulberry

Morus tatarica Pallas, not L. $=$ Morus alba L.

MUHLENBERGIA Schreb. (Poaceae)

*M. asperifolia (Nees \& Meyer) Parodi

M. capillaris (Lam.) Trin., Hairgrass

Muhlenbergia commutata (Scribn.) Bush =

Muhlenbergia frondosa (Poir.) Fern.

Muhlenbergia diffusa Willd. = Muhlenbergia schreberi Gmel.

Muhlenbergia foliosa (R. \& S.) Trin. = Muhlenbergia mexicana (L.) Trin.

M. frondosa (Poir.) Fern., Leafy Muhlenbergia, Wirestem-muhly

= M. commutata (Scribn.) Bush

= M. mexicana of some authors, not (L.)

Trin.

$=$ M. mexicana var. commutata Scribn.

M. glomerata (Willd.) Trin., Marsh-timothy

$=$ M. glomerata var. cinnoides (Link)

Hermann

$=$ M. racemosa of NY reports, not (Michx.) BSP.

$=$ M. racemosa var. violacea Scribn.

M. mexicana (L.) Trin., Wood-grass, Satin-grass, Muhly

$=$ M. foliosa (R. \& S.) Trin.

Muhlenbergia mexicana of some authors, not (L.)

Trin. $=$ Muhlenbergia frondosa (Poir.)

Fern.

Muhlenbergia mexicana var. commutata Scribn. = Muhlenbergia frondosa (Poir.) Fern.

Muhlenbergia racemosa of NY reports, not (Michx.) BSP. $=$ Muhlenbergia glomerata (Willd.) Trin.

Muhlenbergia racemosa var. violacea Scribn. = Muhlenbergia glomerata (Willd.) Trin.

M. schreberi Gmel., Dropseed, Nimble Will $=$ M. diffusa Willd.

M. sylvatica (Torr.) Torr. ex A. Gray, Woodland Dropseed

= M. umbrosa Scribn. 
var. sylvatica

$=$ M. sylvatica var. attenuata Carpenter

M. tenuiflora (Willd.) BSP., Slender Satin-grass $=\mathrm{M}$. willdenovii Trin.

Muhlenbergia umbrosa Scribn. $=$ Muhlenbergia sylvatica (Torr.) Torr. ex A. Gray

Muhlenbergia willdenovii Trin. $=$ Muhlenbergia tenuiflora (Willd.) BSP.

Mulgedium leucophaeum (Willd.) DC. = Lactuca biennis (Moench) Fern.

Muricauda dracontium (L.) Kunth = Arisaema dracontium (L.) Schott ex Schott \& Endl.

MUSCARI Mill. (Liliaceae)

*M. botryoides (L.) Mill., Grape-hyacinth

MYOSOTIS L. (Boraginacaceae)

*M. arvensis (L.) Hill, Field Forget-me-not

M. laxa Lehm., Small Forget-me-not

Myosotis macrophylla Adams $=$ Brunnera macrophylla (Adams) I. M. Johnston

Myosotis micrantha of authors, not Pall. = Myosotis stricta Link ex R. \& S.

Myosotis palustris Lam. $=$ Myosotis scorpioides L.

*M. scorpioides L., European Forget-me-not $=$ M. palustris Lam.

*M. stricta Link ex R. \& S., Forget-me-not $=$ M. micrantha of authors, not Pall.

*M. sylvatica Hoffm., Garden Forget-me-not

M. verna Nutt., Early or Spring Forget-me-not $=$ M. virginica of authors, not (L.) BSP.

Myosotis virginica of authors, not (L.) BSP. = Myosotis verna Nutt.

MYOSOTON Moench (Caryophyllaceae)

*M. aquaticum (L.) Moench, Water Mouse-ear

Chickweed, Giant Chickweed

$=$ Alsine aquatica (L.) Britt.

$=$ Cerastium aquaticum $\mathrm{L}$.

$=$ Malachium aquaticum (L.) Fries

$=$ Stellaria aquatica (L.) Scop.

MYRICA L. (Myricaceae)

Myrica asplenifolia L. = Comptonia peregrina (L.) Coult.

Myrica carolinensis of NY reports, not Mill. = Myrica pensylvanica Loisel. ex Duhamel

Myrica cerifera (of Day, 1882) $=$ Myrica

pensylvanica Loisel. ex Duhamel
M. gale L., Sweet Gale, Meadow-fern

$=$ Gale palustris $($ Lam.) Chev.

$=$ Myrica gale var. subglabra (Chev.) Fern.

$=$ M. palustris Lam.

M. pensylvanica Loisel. ex Duhamel, Bayberry, Candleberry

$=$ Morella caroliniana Small

$=$ Myrica carolinensis of NY reports, not Mill.

$=$ M. cerifera (of Day, 1882)

$=\mathrm{M}$. carolinensis (of authors)

Myrica palustris Lam. $=$ Myrica gale L .

Myrica peregrina (L.) Kuntze $=$ Comptonia peregrina (L.) Coult.

MYRIOPHYLLUM L. (Haloragaceae)

Myriophyllum exalbescens Fern. = Myriophyllum sibericum Komarov

M. heterophyllum Michx., Water-milfoil

$=\mathrm{M}$. hippuroides of NY reports, not Nutt. ex Torrey \& A. Gray

Myriophyllum hippuroides of NY reports, not Nutt. ex Torr. \& A. Gray = Myriophyllum heterophyllum Michx.

M. sibericum Komarov, Water-milfoil

$=$ M. exalbescens Fern.

$=$ M. spicatum L.

$=$ M. spicatum ssp. exalbescens (Fern.) Hultén

$=$ M. spicatum var. exalbescens (Fern.) Jepson

Myriophyllum spicatum L. $=$ Myriophyllum sibericum Komarov

Myriophyllum spicatum ssp. exalbescens (Fern.)

Hultén = Myriophyllum sibericum Komarov

Myriophyllum spicatum var. exalbescens (Fern.) Jepson $=$ Myriophyllum sibericum Komarov

M. verticillatum L., Whorled Water-milfoil $=\mathrm{M}$. verticillatum var. pectinatum Wallr.

Nabalus albus (L.) Hooker $=$ Prenanthes alba L.

Nabalus altissimus (L.) Hooker $=$ Prenanthes altissima $\mathrm{L}$.

Nabalus crepidineus (Michx.) DC. $=$ Prenanthes crepidinea Michx.

Nabalus trifoliolatus Cass. $=$ Prenanthes trifoliata (Cass.) Fern. 
Nabalus trifoliatus of authors $=$ Prenanthes trifoliata (Cass.) Fern.

Nabalus trifoliatus var. dissectifolius Peck $=$ Prenanthes trifoliata (Cass.) Fern.

Naias (orth. var.) = Najas L.

NAJAS L. (Najadaceae)

N. flexilis (Wills.) Rostk. \& Schmidt, Slender Naiad, Bushy Naiad

NARCISSUS L. (Amaryllidaceae)

*N. pseudo-narcissus L., Daffodil, Trumpetnarcissus

Nardosmia palmata Hooker $=$ Petasites frigidus var. palmatus (Ait.) Cronq.

Nardosmia japonica Siebold \& Zucc. $=$ Petasites japonicus (Siebold \& Zuc.) Maxim.

Nasturtium armoracia (L.) Fries = Armoracia rusticana (Lam.) Gaertn., Mey. \& Scherb.

Nasturtium lacustre A. Gray = Armoracia lacustris (A. Gray) Al-Shehbaz \& V. Bates

Nasturtium nasturtium-aquaticum (L.) Karst. = Rorippa nasturtium-aquaticum (L.) Hayek

Nasturtium officinale R. Br. ex Ait. = Rorippa nasturtium-aquaticum (L.) Hayek

Nasturtium palustre (L.) DC. of Zander \& Pierce $(1979)=$ Rorippa palustris (L.) Besser ssp. fernaldiana (Butters \& Abbe) Jonesell

Naumbergia thrysiflora (L.) Duby = Lysimachia thyrsifolia L.

Negundo negundo (L.) Karst. $=$ Acer negundo L.

Neillia opulifolia (L.) Brew. \& Wats. = Physocarpus opulifolius (L.) Maxim.

Nelumbium luteum Willd. $=$ Nelumbo lutea (Willd.) Pers.

NELUMBO Adans. (Nymphaeaceae)

*N. lutea (Willd.) Pers., American Lotus, Yellow Lotus, Wankapin

$=$ Nelumbium luteum Willd.

NEMOPANTHUS Raf. (Aquifoliceae)

Nemopanthus canadensis DC. $=$ Nemopanthus mucronatus (L.) Loesener ex Koehne

N. mucronatus (L.) Loesener ex Koehne, Mountain-holly, Catberry

$=\mathrm{N}$. canadensis DC.

Neobeckia aquatica (Eaton) Greene $=$ Armoracia lacustris (A. Gray) Al-Shehbaz \& V. Bates
NEPETA L. (Lamiaceae)

*N. cataria L., Catnip, Catmint

Nepeta glechoma Benth. = Glechoma hederacea L.

Nepeta hederacea (L.) Trev. = Glechoma hederacea L.

Nephrodium acrostichoides Michx. $=$ Polystichum acrostichoides (Michx.) Schott

Nephrodium punctilobulum Michx. $=$ Dennstaedtia punctilobula (Michx.) Moore

Nesaea verticillata (L.) HBK = Decodon verticillatus (L.) Ell.

NICANDRA Adans. (Solanaceae)

*N. physalodes (L.) Gaertn., Apple of Peru

= Atropa pysalodes L.

$=$ Physalodes Physalodes (L.) Britt.

NICOTIANA L. (Solanaceae)

*N. longiflora Cav., Long-flowered Tobacco

*N. rustica L., Indian Tobacco

NIGELLA L. (Ranunculaceae)

*N. damascena L., Love-in-a-mist, Fennel-flower

"Native of the Old World and frequent in cultivation. Reported by Day (Plants of Buffalo 14, 1883) as spontaneous in gardens and by Clinton (1.c.Day), as an escape." (House 1924).

Norta altissima (L.) Britt. ex Britt. \& Brown = Sisymbrium altissimum L.

Notholcus lanatus (L.) Nash ex Hitchc. in Jepson

NUPHAR J. Smith ex Sibth. \& Sm. (Nymphaeaceae)

N. advena (Soland. ex Ait.) R. Br. ex Ait. f., Yellow Pondlily, Yellow Cowlily, Spatterdock, Bull-head Lily.

$=$ Nuphar fluviatile (Harper) Standl.

$=$ N. lutea (L.) Sibth. \& Sm. ssp. advena (Ait.) Kartesz \& Gandhi

$=$ N. lutea ssp. macrophylla (Small) Beal

= Nymphozanthus advena (Ait.) Fern.

= Nymphaea advena Soland. ex Ait.

Nuphar advena var. variegata (Engelm. ex Durand in Clinton) A. Gray = Nuphar variegata

Engelm. ex Durand in Clinton

Nuphar fluviatile (Harper) Standl. = Nuphar advena (Soland. ex Ait.) R. Br. ex Ait. f. 
Nuphar lutea (L.) Sibth. \& Sm. ssp. advena (Ait.) Kartesz \& Gandhi $=$ Nuphar advena (Soland. ex Ait.) R. Br. ex Ait. f.

Nuphar lutea ssp. macrophylla $($ Small $)$ Beal $=$ Nuphar advena (Soland. ex Ait.) R. Br. ex Ait. f.

Nuphar lutea (L.) Sibth. \& Sm. ssp. pumila (Timm) Beal $=$ Nuphar microphylla (Pers.) Fern.

Nuphar lutea (L.) Sibth. \& Sm. ssp. variegata (Engelm. ex Durand in Clinton) Beal = Nuphar variegata Engelm. ex Durand in Clinton

N. microphylla (Pers.) Fern., Small Yellow Pondlily, Small Yellow Cow-lily, Dwarf Spatterdock.

$=$ N. lutea (L.) Sibth. \& Sm. ssp. pumila (Timm) Beal

$=$ N. pumila $($ Timm $)$ DC.

$=$ Nymphaea lutea [beta sign] microphylla Pers.

$=$ Nymphaea microphylla (Pers.) Robins. \& Fern.

Nuphar pumila $($ Timm $)$ DC. $=$ Nuphar microphylla (Pers.) Fern.

N. variegata Engelm. ex Durand in Clinton, Common Yellow Cow-lily, Yellow Pondlily, Spatterdock

$=\mathrm{N}$. advena var. variegata (Engelm. ex Durand in Clinton) A. Gray

$=$ N. lutea (L.) Sibth. \& Sm. ssp. variegata (Engelm. ex Durand in Clinton) Beal

= Nymphaea advena var. variegata (Engelm. ex Durand in Clinton) Fern.

= Nymphozantus variegatus (Engelm.) Fern.

NYMPHAEA L. (Nymphaeaceae)

Nymphaea advena Soland. ex Ait. $=$ Nuphar advena (Soland. ex Ait.) R. Br. ex Ait. f.

Nymphaea advena var. variegata (Engelm. ex Durand in Clinton) Fern. $=$ Nuphar variegata Engelm. ex Durand in Clinton

Nymphaea lekophylla (Small) Coryl = Nymphaea odorata Dryand. ex Ait. ssp. odorata

Nymphaea lutea [beta sign] microphylla Pers. = Nuphar microphylla (Pers.) Fern.

Nymphaea microphylla (Pers.) Robins. \& Fern. = Nuphar microphylla (Pers.) Fern.

N. odorata Dryand. ex Ait., Fragrant White Water-lily ssp. odorata

$=$ Castalia odorata (Dryand ex Ait.) Woodr. \& Wood.

$=$ C. lekophylla Small

= Nymphaea odorata var. gigantea Tricker

$=$ N. odorata var. minor Sims

$=$ N. odorata var. rubra Pursh

$=$ N. lekophylla (Small) Coryl

ssp. tuberosa (Paine) Wiersma \& Hellquist

$=$ Castalia tuberosa (Paine) Greene

= Nymphaea tuberosa Paine

Nymphaea odorata var. gigantea Tricker $=$ Nymphaea odorata Dryand. ex Ait.

Nymphaea tuberosa Paine $=$ Nymphaea odorata

Dryand. ex Ait. ssp. tuberosa (Paine)

Wiersma \& Hellquist

Nymphozanthus advena (Ait.) Fern. $=$ Nuphar advena (Soland. ex Ait. R. Br. ex Ait. f.

Nymphozantus variegatus (Engelm.) Fern. = Nuphar variegata Engelm. ex Durand in Clinton

Nyssa multiflora Wang = Nyssa sylvatica Marsh .

NYSSA L. (Nyssaceae)

Nyssa multiflora Wang = Nyssa sylvatica Marsh .

N. sylvatica Marsh., Tupelo, Black Gum, Pepperidge, Sour Gum $=\mathrm{N}$. multiflora Wang

Oakesia sessilifolia (L.) S. Wats. = Uvularia sessilifolia L.

Oclemena acuminata $($ Michx. $)$ Greene $=$ Aster acuminatus Michx.

Oclemena nemoralis (Ait.) Greene $=$ Aster nemoralis Ait.

OENOTHERA L. (Onagraceae)

O. biennis L., Common Evening-primose

$=\mathrm{O}$. chrysantha Michx.

$=$ O. biennis var. nutans (Atkins. \& Bartl.) Wieg.

$=$ O. biennis var. pycnocarpa (Atkins. \&

Bartl.) Wieg.

$=$ O. biennis ssp. muricata (L.) Piper

$=$ O. erythrosepala Borbás

$=$ O. glazioviana Micheli

$=\mathrm{O}$. muricata $\mathrm{L}$.

Oenthera chrysantha Michx. $=$ Oenothera biennis L. 
Oenothera cleistantha Shull \& Bartlett = Oenothera parviflora L. var. parviflora

Oenothera cruciata Nutt. ex G. Don in part, not (S. Wats.) Munz $=$ Oenothera parviflora L. var. parviflora

Oenothera erythrosepala Borbás = Oenothera biennis L.

O. fruticosa L., Sundrops ssp. glauca (Michx.) Straley

$=$ Kneiffia tetragona (Roth) Pennell

$=$ Oenothera tetragona Roth

Oenothera glazioviana Micheli $=$ Oenothera biennis L.

O. laciniata Hill, Cut-leaved Evening-primose $=$ Raimannia laciniata (Hill) Rose

Oenothera muricata L. = Oenothera biennis L.

Oenothera muricata of some NY reports, not L. = Oenothera parviflora L. var. parviflora

Oenothera oakesiana (A. Gray) Robbins ex S. Wats. \& Coult. = Oenothera parviflora L. var. oakesiana (A. Gray) Fern.

O. parviflora L., Small-flowered Eveningprimrose

var. oakesiana (A. Gray) Fern.

$=$ O. oakesiana (A. Gray) Robbins ex S. Wats. \& Coult.

$=\mathrm{O}$. parviflora ssp. angustissima (Gates) Muhz

var. parviflora

$=$ O. cleistantha Shull \& Bartlett

$=$ O. cruciata Nutt. ex G. Don in part, not (S. Wats.) Munz

$=\mathrm{O}$. muricata of some NY reports, not L.

$=\mathrm{O}$. parviflora of authors, not L.

O. perennis L., Small Sundrops

$=$ Kneiffia perennis (L.) Pennell

$=$ Oenothera perennis var. receptilis Blake

$=\mathrm{O}$. pumila $\mathrm{L}$.

Oenothera perennis var. receptilis Blake $=$ Oenothera perennis L.

*O. pilosella Raf., Prairie-sundrops

$=$ Kneiffia pratensis Small

$=$ Oenothera pratensis (Small) B. Robinson

Oenothera pratensis $($ Small $)$ B. Robinson $=$

Oenothera pilosella Raf.

Oenothera pumila L. = Oenothera perennis L.

Oenothera tetragona Roth $=$ Oenothera fruticosa L . ssp. glauca (Michx.) Straley
Oldenlandia caerulea (L.) Gray = Houstonia caerulea L.

ONOBRYCHIS P. Mill. (Fabaceae)

Onobrychis onobrychis (L.) Harst., not Rydb. = Onobrychis viciifolia Scop.

Onobrychis sativa Lam. $=$ Onobrychis viciifolia Scop.

*O. viciifolia Scop., Sainfoin, Holly-clover

$=$ Hedysarum onobrychis L.

= Onobrychis onobrychis (L.) Harst., not Rydb.

= O. sativa Lam.

ONOCLEA L. (Dryopteridaceae)

O. sensibilis L., Sensitive Fern

ONOPORDUM L. (Asteraceae $)=$ Onopordon (Vaill.) L. of authors

*O. acanthium L., Scotch Thistle, Cotton-thistle

ONOSMODIUM Michx. (Boraginaceae)

Onosmodium carolinianum (of authors) $=$ Onosmodium molle Michx. ssp. hispidissimum (Mackz.) Boivin

Onosmodium hispidissimum Mackz. = Onosmodium molle Michx. ssp. hispidissimum (Mackz.) Boivin

O. molle Michx., False Gromwell, Marble-seed, Gravel-weed

ssp. hispidissimum (Mackz.) Boivin

$=$ O. carolinianum (of authors)

$=\mathrm{O}$. hispidissimum Mackz.

= O. molle var. hispidissimum (Mackz.) Cronq.

Onosmodium molle Michx. var. hispidissimum (Mackz.) Cronq. $=$ Onosmodium molle Michx. ssp. hispidissimum (Mackz.) Boivin

O. virginianum (L.) A. DC., Virginia False Gromwell

OPHIOGLOSSUM L. (Ophioglossaceae)

O. pusillum Raf., Adder's-tongue Fern $=\mathrm{O}$. vulgatum var. pseudopodum (S. Blake) Farw.

$=\mathrm{O}$. vulgatum var. vulgatum of authors, not L. 
Ophrys auriculata (Wieg.) House = Listera australis Lindl.

Oplotheca gracilis Hooker $=$ Froelichia gracilis (Hooker) Moq.

Opulaster intermedius Rydb. ex Britt. = Physocarpus opulifolius (L.) Maxim. var. intermedius (Rydb.) Robins.

Opulaster opulifolius (L.) Kuntze $=$ Physocarpus opulifolius (L.) Maxim. var. opulifolius

Orchis spectabilis L. $=$ Galearis spectabilis (L.) Raf.

ORIGANUM L. (Lamiaceae)

*O. vulgare L., Wild Marjoram, Oregano

ORNITHOGALUM L. (Liliaceae)

*O. nutans L., Nodding Star-of-Bethlehem

*O. umbellatum L., Star-of-Bethlehem

Orobanche americana L. $=$ Conopholis americana (L.) Wallr.

OROBANCHE L. (Orobanchaceae)

O. uniflora L., One-flowered Cancer-root = Aphyllon uniflorum (L.) Torr. \& A. Gray ex A. Gray

$=$ Thalesia uniflora (L.) Britt.

Orobanche virginiana L. = Epifagus virginiana (L.) Bart.

ORTHILIA Raf. (Ericaceae)

O. secunda (L.) House, One-sided Pyrola $=$ O. secunda ssp. obtusata (Turcz.) Böcher $=$ O. secunda var. obtusata (Turcz.) House $=$ Pyrola secunda $\mathrm{L}$.

$=$ P. secunda var. obtusata Turcz.

$=\mathrm{P}$. secunda var. pumila Paine

ORYZOPSIS Michx. (Poaceae)

O. asperifolia Michx., Mountain-rice, Spreading Ricegrass, Whitegrass

Oryzopsis canadensis sensu Torrey's Flora of NY, not (Poir.) Trin. $=$ Oryzopsis pungens (Torr. ex Spreng.) Hitchc.

Oryzopsis melanocarpa Muhl. $=$ Oryzopsis racemosa (Sm.) Ricker ex Hitchc.

O. pungens (Torr. ex Spreng.) Hitchc., Slender Mountain-rice

$=$ O. canadensis sensu Torrey's Flora of NY, not (Poir.) Trin.
= Piptatherum pungens (Torr. ex Spreng.)

Dorn

O. racemosa (Sm.) Ricker ex A. S. Hitchc., Blackfruited Mountain-rice

$=$ O. melanocarpa Muhl.

= Piptatherum racemosum Ricker ex A. S. Hitchc.

OSMORHIZA Raf. (Apiaceae)

Osmorhiza brevistylis DC. $=$ Osmorhiza claytonii (Michx.) Clarke

O. claytonii (Michx.) Clarke, Hairy Sweet Cicely, Sweet Jarvil

$=\mathrm{O}$. brevistylis DC.

O. longistylis (Torr.) DC., Long-styled Sweet Cicely

$=\mathrm{O}$. longistylis varieties of NY reports

$=\mathrm{O}$. longistylis var. brachycoma Blake

OSMUNDA L. (Osmundaceae)

O. cinnamomea L., Cinnamon-fern

O. claytoniana L., Interrupted Fern

O. regalis L., Royal Fern, Flowering Fern var. spectabilis (Willd.) A. Gray

$=\mathrm{O}$. spectabilis Willd.

Osmunda spectabilis Willd. = Osmunda regalis L., var. spectabilis (Willd.) A. Gray

Osmunda struthiopteris L. = Matteuccia struthiopteris (L.) Todaro

Osteospermum canadense (L.) House = Polymnia canadensis L.

Osteospermum uvedalia L. = Polymnia uvedalia (L.) L.

OSTRYA Scop. (Betulaceae)

O. virginiana (Mill.) K. Koch, Hop- or American Hornbeam, Ironwood, Blue Beech, Waterbeech, Musclewood

$=$ Carpinus virginiana Mill.

= Ostrya virginiana var. glandulosa Sarg.

$=$ O. virginiana var. lasia Fern.

OXALIS L. (Oxalidaceae)

Oxalis acetosella of American authors, not L. = Oxalis montana Raf.

Oxalis acetosella var. montana (Raf.) Hultén ex D. Löve $=$ Oxalis montana Raf.

Oxalis bushii (Small) Small $=$ Oxalis stricta L . 
*O. corniculata L., Creeping Yellow Wood-

sorrel, Lady's Sorrel

$=$ O. repens Thunb.

$=\mathrm{O}$. corniculata var. viscidula Wieg.

Oxalis europaea Jord. $=$ Oxalis stricta L .

Oxalis fontana Bunge $=$ Oxalis stricta $\mathrm{L}$.

O. montana Raf., Common Wood-sorrel, American Shamrock

$=\mathrm{O}$. acetosella of American authors, not L.

$=$ O. acetosella var. montana (Raf.) Hultén ex

D. Löve

$=$ O. montana Raf.

Oxalis oneidica House $=$ Oxalis stricta L.

Oxalis repens Thunb. = Oxalis corniculata L.

Oxalis rufa Small ex Britt. = Oxalis stricta L.

O. stricta L., Upright Yellow Wood-sorrel

$=$ O. bushii (Small) Small

$=$ O. europaea Jord.

$=\mathrm{O}$. fontana Bunge

$=\mathrm{O}$. oneidica House

$=$ O. rufa Small ex Britt.

O. violacea L., Violet Wood-sorrel

Oxybaphus nyctagineus (Michx.) Sweet $=$ Mirabilis nyctaginea (Michx.) MacM.

Oxycoccus macrocarpus (Ait.) Pursh = Vaccinium macrocarpon Ait.

OXYPOLIS Raf. (Apiaceae)

O. rigidior (L.) Raf., Cowbane, Water-dropwort

$=\mathrm{O}$. rigidior var. longifolius (Pursh) Britt.

$=$ O. turgida Small

Packera aurea (L.) A. \& D. Löve $=$ Senecio aureus L.

Packera obovata (Muhl. ex Willd.) W. A. Weber \&

A. Löve $=$ Senecio obovatus Muhl. ex Wild.

Packera paupercula (Michx.) Á. \& D. Löve = Senecio pauperculus Michx.

PANAX L. (Araliaceae)

P. quinquefolius L., Ginseng, Sang

$=$ Aralia quinquefolia (L.) Dec.\& Planche.

P. trifolius L., Dwarf Ginseng, Ground-nut

$=$ Aralia trifolia (L.) Dec. \& Planch

Panicularia borealis Nash $=$ Glyceria borealis (Nash) Batchelder

Panicularia canadensis (Michx.) Kuntze = Glyceria canadensis (Michx.) Trin.
Panicularia grandis (S. Wats.) Nash = Glyceria grandis S. Wats.

Panicularia melicaria (Michx.) Hitchc. = Glyceria melicaria (Michx.) Hubbard

Panicularia nervata (Willd.) Kuntze $=$ Glyceria striata (Lam.) Hitchc.

Panicularia septentrionalis (Hitchc.) Bickn. = Glyceria septentrionalis Hitchc.

PANICUM L. (Poaceae)

P. acuminatum Sw. (sensu lato), Panic-grass

$=$ Dichanthelium acuminatum (Sw.) Gould \& Clark

$=$ Panicum auburne Ashe

$=$ P. huachucae Ashe

= P. huachucae var. sylvicola Hitch. \& Chase

= P. implicatum Scribn.

$=\mathrm{P}$. lanuginosum Ell., in part

$=\mathrm{P}$. lanuginosum var. fasciculatum (Torr.)

Fern.

$=\mathrm{P}$. lanuginosum var. implicatum (Scribn.)

Fern.

$=\mathrm{P}$. lanuginosum var. lindheimeri (Nash)

Fern.

$=\mathrm{P}$. lanuginosum var. septentrionale Fern.

$=\mathrm{P}$. lindheimeri Nash

$=\mathrm{P}$. lindheimeri var. fasciculatum (Torr.)

Fern.

$=\mathrm{P}$. lindheimeri var. implicatum (Scribn.)

Fern.

$=\mathrm{P}$. lindheimeri Nash var. lindheimeri

(Nash) Fern.

$=\mathrm{P}$. lindheimeri var. septentrionale Fern.

$=\mathrm{P}$. meridionale Ashe

$=\mathrm{P}$. nitidum Lam.

$=$ P. spretum Schultes

$=\mathrm{P}$. subvillosum Ashe

$=\mathrm{P}$. tennesseense Nash

$=\mathrm{P}$. villosissimum Nash

$=\mathrm{P}$. wrightianum Scribn.

Panicum agrostoides Spreng. $=$ Panicum rigidulum

Bosc ex Nees

Panicum annulum Ashe $=$ Panicum dichotomum L.

Panicum ashei Pearson ex Ashe $=$ Panicum

commutatum Schultes

Panicum auburne Ashe $=$ Panicum acuminatum

Sw.

Panicum barbipulvinatum Nash $=$ Panicum

capillare L. 
Panicum barbulatum Michx. $=$ Panicum dichotomum L.

Panicum bicknellii var. calliphyllum (Ashe)

Gleason $=$ Panicum boreale Nash

P. boreale Nash, Northern Panic-grass

$=$ Dichanthelium boreale (Nash) Freckmann

$=\mathrm{P}$. bicknellii var. calliphyllum (Ashe)

Gleason

$=\mathrm{P}$. calliphyllum Ashe

Panicum boscii Poir. ex Lam. $=$ Panicum latifolium

L.

Panicum calliphyllum Ashe $=$ Panicum boreale Nash

P. capillare L., Witchgrass

$=\mathrm{P}$. capillare varieties of $\mathrm{NY}$ reports

$=\mathrm{P}$. barbipulvinatum Nash

P. clandestinum L., Deer-tongue Grass

$=$ Dichanthelium clandestinum (L.) Gould

= Panicum latifolium L. var. clandestinum (L.) Pursh

Panicum clutei Nash $=$ Panicum dichotomum L.

P. commutatum Schultes, Ashe's Panic-grass

$=$ Dichanthelium commutatum (Schultes) Gould

$=$ Panicum ashei Pearson ex Ashe

$=\mathrm{P}$. commutatum var. ashei (Pearson ex

Ashe) Fern.

$=\mathrm{P}$. umbrosum Le Conte

Panicum crusgalli L. = Echinochloa crusgalli (L.)

Beauv. ssp. crusgalli

P. depauperatum Muhl., Starved Panic-grass

= Dichanthelium depauperatum (Muhl.)

Gould

= Panicum depauperatum var. psilophyllum Fern.

$=\mathrm{P}$. depauperatum var. involutum (Torr.) A. Wood

Panicum depauperatum var. psilophyllum Fern. = Panicum depauperatum Muhl.

P. dichotomiflorum Michx., Spreading Witchgrass, Smooth Panic-grass, Fall Panicgrass, Fall Panicum

var. dichotomiflorum

$=\mathrm{P}$. dichotomiflorum var. geniculatum (Wood) Fern.

Panicum dichotomiflorum var. geniculatum

(Wood) Fern. $=$ Panicum dichotomiflorum Michx.

P. dichotomum L., Forked Panic-grass
$=$ Dichanthelium dichotomum (L.) Gould

$=\mathrm{P}$. annulum Ashe

$=\mathrm{P}$. barbulatum Michx.

$=\mathrm{P}$. dichotomum var. barbulatum (Michx.)

Wood

$=$ P. clutei Nash

$=$ P. lucidum Ashe

$=\mathrm{P}$. mattamuskeetense Ashe

$=$ P. microcarpon Muhl. ex Ell.

$=\mathrm{P}$. nitidum Lam.

P. flexile (Gatt.) Scribn., Wiry Witch-grass, Panicgrass

P. gattingeri Nash, Gattinger's Witch-grass $=\mathrm{P}$. capillare var. campestre Gatt.

Panicum glabrum (Schrad.) Gaud. = Digitaria ischaemum (Schreb. ex Schweig.) Schreb. ex Muhl.

Panicum huachucae Ashe $=$ Panicum acuminatum Sw.

Panicum huachucae var. sylvicola Hitch. \& Chase $=$ Panicum acuminatum Sw.

Panicum implicatum Scribn. $=$ Panicum acuminatum Sw.

Panicum lanuginosum Ell., in part = Panicum acuminatum Sw.

Panicum lanuginosum var. fasciculatum (Torr.) Fern. $=$ Panicum acuminatum Sw.

Panicum lanuginosum var. implicatum (Scribn.) Fern. $=$ Panicum acuminatum Sw.

Panicum lanuginosum var. lindheimeri (Nash) Fern. $=$ Panicum acuminatum Sw.

Panicum lanuginosum Ell. var. praecocius (Hitchc. $\&$ Chase) Dore $=$ Panicum villosissimum Nash

Panicum lanuginosum var. septentrionale Fern. = Panicum acuminatum Sw.

P. latifolium L., Broad-leaved Panic-grass

= Dichanthelium latifolium (L.) Gould \& C. Clark

$=$ P. boscii Poir. ex Lam.

Panicum lindheimeri var fasciculatum (Torr.) Fern. $=$ Panicum acuminatum Sw.

Panicum lindheimeri var. implicatum (Scribn.) Fern. $=$ Panicum acuminatum Sw .

Panicum lindheimeri var. lindheimeri Nash $=$ Panicum acuminatum Sw.

Panicum lindheimeri var. septentrionale Fern. = Panicum acuminatum Sw. 
P. linearifolium Scribn. ex Nash in Britt. \& Brown, Low White-haired Panic-grass

$=$ Dichanthelium linearifolium (Scribn. ex Nash) Gould

$=$ Panicum linearifolium var. werneri (Scribn.) Fern.

$=\mathrm{P}$. perlongum Nash

$=$ P. werneri Scribn.

Panicum longifolium Torr. $=$ Panicum rigidulum

Bosc. ex Nees

Panicum lucidum Ashe $=$ Panicum dichotomum L.

Panicum macrocarpon Torr. $=$ Panicum oligosanthes Schult.

Panicum mattamuskeetense Ashe $=$ Panicum dichotomum L.

Panicum microcarpon Muhl. ex Ell. = Panicum dichotomum L.

*P. miliaceum L., Broomcorn-millet, Proso Millet Panicum nitidum Lam. = Panicum dichotomum L.

P. oligosanthes Schult., Few-flowered Panic-grass var. scribnerianum (Nash) Fern.

$=$ Dichanthelium oligosanthes $(\mathrm{J}$. A.

Schultes) Gould var. scribnerianum (Nash) Gould

$=$ P. scribnerianum Nash

$=$ P. macrocarpon Torr. of Zander and Pierce (1979)

Panicum perlongum Nash $=$ Panicum linearifolium Scribn. ex Nash in Britt. \& Brown

*P. philadelphicum Bernh., Wood Witch-grass, Philadelphia Witch-grass

Panicum praecocius Hitchc. $\&$ Chase $=$ Panicum villosissimum Nash

Panicum pseudopubescens Nash $=$ Panicum villosissimum Nash

P. rigidulum Bosc ex Nees, Redtop Panic-grass var. rigidulum

$=\mathrm{P}$. agrostoides Spreng.

Panicum sanguinale L. = Digitaria sanguinalis (L.) Scop.

P. sphaerocarpon Ell., Round-fruited Panic-grass var. sphaerocarpon

$=$ Dichanthelium spaerocarpon (Ell.) Gould var. sphaerocarpon

Panicum tennesseense Nash $=$ Panicum acuminatum $\mathrm{Sw}$.

Panicum umbrosum Le Conte $=$ Panicum commutatum Schultes

P. villosissimum Nash, Panic Grass
$=$ Dichanthelium villosissimum (Nash)

Freckman var. praecocius (Hitchc. \&

Chase) Freckmann

$=$ Dichanthelium acuminatum (Sw.) Gould \&

Clark var. villosum (Gray) Gould \& Clark

$=$ Panicum lanuginosum Ell. var. praecocius

(Hitchc. \& Chase) Dore

$=$ P. praecocius Hitchc. $\&$ Chase

$=$ P. pseudopubescens Nash

P. virgatum L., Switch-grass

var. virgatum

$=\mathrm{P}$. virgatum var. cubense Griseb.

Panicum werneri Scribn. $=$ Panicum linearifolium Scribn. ex Nash

Panicum xalapense HBK = Panicum laxiflorum

Lam. (excluded by Zander \& Pierce 1979)

P. xanthophysum A. Gray, Slender Panic-grass

$=$ Dichanthelium xanthophysum (A. Gray)

Freckman

PAPAVER L. (Papaveraceae)

*P. rhoeas L., Corn-poppy, Shirley-poppy, Fieldpoppy

*P. somniferum L., Opium-poppy

PARNASSIA L. (Saxifragaceae)

Parnassia americana Muhl. = Parnassia glauca Raf. Parnassia caroliniana of NY reports, not Michx. = Parnassia glauca Raf.

P. glauca Raf., Grass-of-Parnassus

$=\mathrm{P}$. americana Muhl.

$=\mathrm{P}$. caroliniana of NY reports, not Michx.

$=\mathrm{P}$. palustris of NY report, not L.

Parnassia palustris of NY report, not L. = Parnassia glauca Raf.

Parsonsia petiolata (L.) Rusby $=$ Cuphea viscosissima Jacq.

PARONYCHIA (Caryophyllaceae)

P. fastigiata (Raf.) Fern., Forked Chickweed $=$ Anychia divaricata Raf.

$=$ A. polygonoides Raf.

$=$ Paronychia fastigiata var. nuttallii of NY reports, not (Small) Fern.

PARTHENIUM L. (Asteraceae)

P. integrifolium L., Wild Quinine

PARTHENOCISSUS Planch. ex DC. (Vitaceae) 
Parthenocissus inserta (Knerr) Fritsch $=$ Parthenocissus vitacea (L.) Planch. ex DC.

P. quinquefolia (L.) Planch. ex DC., Virginia Creeper, Woodbine

= Ampelopsis quinquefolia (L.) Michx.

$=$ Parthenocissus quinquefolia var. hirsuta Pursh

$=$ Psedera quinquefolia (L.) Greene

$=\mathrm{P}$. quinquefolia var. hirsuta (Pursh) B. Robinson

*P. tricuspidata (Sieb. \& Zucc.) Planch. ex DC., Boston Ivy, Japanese Ivy, Cottage-ivy

P. vitacea (L.) Planch. ex DC., Discless Virginia Creeper, Woodbine

$=$ P. inserta (Knerr) Fritsch

PASCOPYRUM Á. Löve (Poaceae)

*P. smithii (Rydb.) Á. Löve, Blue-joint, Western Wheat-grass

$=$ Agropyron smithii Rydb.

PASTINACA L. (Apiaceae)

*P. sativa L., Parsnip

PEDICULARIS L. (Scrophulariaceae)

P. canadensis L., Common Lousewort, Woodbetony

$=\mathrm{P}$. canadensis var. dobbsii Fern.

P. lanceolata Michx., Swamp Lousewort, Woodbetony

PELLAEA Link (Pteridaceae)

P. atropurpurea (L.) Link, Purple Cliff-brake $=$ Allosorus atropurpureus (L.) Kunze ex Presl

$=$ Pellaea atropurpurea var. cristata Trel.

P. glabella Mett. ex Kuhn, Smooth Cliff-brake $=\mathrm{P}$. atropurpurea var. bushii Mackz.

PELTANDRA Raf. (Araceae)

P. virginica (L.) Schott ex Schott \& Endl. (not Kunth) Green Water-arum, Arrowleaf, Tuckahoe

Pennisetum glaucum (L.) R. Br. = Setaria pumila (Poir.) Schultes

PENSTEMON Mitchell ex Schmid. (Scrophulariaceae)

*P. calycosus Small, Penstemon, Beard-tongue
$=$ P. laevigatus Ait. var. calycosus (Small)

Bennett

P. digitalis Nutt., Foxglove Beard-tongue $=\mathrm{P}$. laevigatus of NY reports, not Ait.

$=\mathrm{P}$. laevigatus var. digitalis (Nutt.) Gray

$=\mathrm{P}$. penstemon of NY reports, not (L.) Britt.

P. hirsutus (L.) Willd., Hairy Beard-tongue $=\mathrm{P}$. pubescens Ait.

Penstemon laevigatus var. digitalis (Nutt.) Gray $=$ Penstemon digitalis Nutt.

(*)P. pallidus Small, Pale Beard-tongue (as native NYFA July 2005)

$=\mathrm{P}$. canescens Britt.

Penstemon penstemon of NY reports, not (L.)

Britt. $=$ Penstemon digitalis Nutt.

Penstemon pubescens Ait. $=$ Penstemon hirsutus (L.) Willd.

Pentaphylloides floribunda (Pursh) Á. Löve = Potentilla fruticosa ssp. floribunda (Pursh) Elkington

PENTHORUM L. (Crassulaceae)

P. sedoides L., Ditch-stoncrop

Pentstemon $($ orth. var. $)=$ Penstemon

Pepo moschatus (Dcne. ex Lam.) Britt. = Cucurbita pepo L.

Pepo pepo (L.) Britt. ex Small = Cucurbita pepo L. Pepo vulgaris (L.) Moench $=$ Cucurbita pepo L.

Peramium pubescens (Willd.) Macm. = Goodyera pubescens (Willd.) R. Br.

Peramium repens (L.) Salisb. $=$ Goodyera repens (L.) R. Br.

Peramium secundum (Raf.) House $=$ Goodyera repens (L.) R. Br.

PERIPLOCA L. (Asclepiadaceae)

*P. graeca L., Silky-vine

Persicaria amphibia (L.) S. Gray = Polygonum amphibium L.

Persicaria arifolia (L.) Haraldson = Polygonum arifolium L.

Persicaria hydropiper (L.) Opiz. = Polygonum hydropiper L.

Persicaria hydropiperoides (Michx.) Small = Polygonum hydropiperoides Michx. var. hydropiperoides

Persicaria lapathifolia (L.) Small [(L.) S. F. Gray sec. NYFA 2005] = Polygonum lapathifolium L. 
Persicaria maculosa S. F. Gray = Polygonum persicaria $\mathrm{L}$.

Persicaria orientalis (L.) Spach = Polygonum orientale L.

Persicaria pensylvanica (L.) Small = Polygonum pensylvanicum L.

Persicaria persicaria (L.) Small $=$ Polygonum persicaria L.

Persicaria punctata (Ell.) Small $=$ Polygonum punctatum Ell. var. punctatum

Persicaria robustior $($ Small) Bickn. $=$ Polygonum robustius (Small) Fern.

Persicaria sagittata (L.) H. Gross = Polygonum sagittata L.

Persicaria setacea (Baldw. ex Ell.) Small = Polygonum setaceum Baldw. ex Ell. var. interjectum Fern.

Persicaria virginiana (L.) Gaert. = Polygonum virginianum $\mathrm{L}$.

Perularia flava (L.) Farw. of NY reports $=$ Platanthera flava (L.) Lindl. var. herbiola (R. Br.) Luer

PETASITES Mill. (Asteraceae)

P. frigidus (L.) Fries, Sweet Coltsfoot

var. palmatus (Ait.) Cronq.

$=$ Nardosmia palmata Hooker

$=$ Petasites palmatus (Ait.) A. Gray ex Brew. \& Watson

$=$ P. vitifolius E. Greene (Morton \& Venn

(1990), s.l.

=Tussilago frigida L. (Morton \& Venn (1990), s.1.

*P. hybridus (L.) Gaertn., Mey \& Scherb., Butterfly-dock

$=\mathrm{P}$. vulgaris Hill

Petasites hybridus as to ON reports, not of (L.) P. Gaertner, Meyer \& Scherb. = Petasites japonicus (Siebold \& Zucc.) Maxim., (Morton \& Venn (1990).

*P. japonicus (Siebold \& Zucc.) Maxim., Japanese Butterflydock, reports from Dufferin Islands, Niagara Falls, Ontario. $=$ Nardosmia japonica Siebold \& Zucc. $=$ Petasites hybridus as to ON reports, not of (L.) P. Gaertner, Meyer \& Scherb. $=\mathrm{P}$. vulgaris as to ON reports, not of Hill
Petasites palmatus (Ait.) A. Gray ex Brew. \& Watson $=$ Petasites frigidus (L.) Fries var. palmatus (Ait.) Cronq.

Petasites vitifolius E. Greene (Morton \& Venn $(1990)=$ Petasites frigidus (L.) Fries, s.l. Petasites vulgaris Hill $=$ Petasites hybridus (L.) Gaertn., Mey. \& Scherb.

Petasites vulgaris as to $\mathrm{ON}$ reports, not of Hill $=$ Petasites japonicus (Siebold \& Zucc.) Maxim. (Morton \& Venn (1990).

Phaca canadensis (L.) Kuntze $=$ Astragalus canadensis L.

Phaca neglecta Torr. \& A. Gray = Astragalus neglectus Torr. \& A. Gray

PHALARIS L. (Poaceae)

P. arundinacea L., Reed Canary-grass $=\mathrm{P}$. arundinacea var. picta $\mathrm{L}$.

*P. canariensis L., Canary-grass

Pharbitis purpurea (L.) Voigt = Ipomoea purpurea (L.) Roth

PHEGOPTERIS Fée (Thelypteridaceae)

P. connectilis (Michx.) Watt, Northern Beech-fern $=$ Dryopteris phegopteris $(\mathrm{L}$.$) Christ.$

$=$ Phegopteris polypodioides Fée

$=$ Phegopteris polypodioides Fée

$=$ Polypodium connectile Michx.

$=\mathrm{P}$. phegopteris L.

$=$ Thelypteris phegopteris (L.) Slosson ex Rydb.

Phegopteris dryopteris (L.) Fée = Gymnocarpium dryopteris (L.) Newm.

P. hexagonoptera (Michx.) Fée, Broad Beech-fern $=$ Dryopteris hexagonoptera (Michx.) Christ. = Polypodium hexagonopterum Michx.

$=$ Thelypteris hexagonoptera (Michx.) Nieul.

Phegopteris polypodioides Fée $=$ Phegopteris connectilis (Michx.) Watt

PHILADELPHUS L. (Hydrangeaceae)

*P. coronarius L., Garden Syringa, European Mock-orange

Philotria canadensis (L. Rich. ex Michx.) Britt. = Elodea canadensis Rich. ex Michx.

Philotria occidentalis (Pursh) House = Elodea canadensis Rich. ex Michx.

Philotria planchonii (Casp.) Rydb. = Elodea canadensis Rich. ex Michx. 
PHLEUM L. (Poaceae)

*P. pratense L., Timothy

*ssp. pratense

Phleum pratense var. nodosum (L.) Huds. = Phleum pratense L. ssp. nodosum (L.) Arcang.

PHLOX L. (Polemoniaceae)

P. divaricata L., Blue Phlox, Wild Sweet-william Phlox divaricata var. lamphamii Wood (excluded by Zander \& Pierce, 1979)

P. maculata L., Wild Sweet William $=$ P. maculata var. candida Michx.

*P. paniculata L., Garden Phlox, Fall or Summer Phlox, Perennial Phlox

P. pilosa L., Downy Phlox $=$ P. pilosa var. virens (Michx.) Wherry

*P. subulata L., Moss-pink, Moss-phlox, Mountain-phlox

Photinia floribunda (Lindl.) Robertson \& Phipps = [Aronia arbutifolia $\times$ melanocarpa $=\mathrm{A} . \times$ prunifolia (Marsh.) Rehd.

Photinia melanocarpa (Michx.) Robertson \& Phipps $=$ Aronia melanocarpa (Michx.) Ell.

Photinia pyrifolia (Lam.) Robertson \& Phipps = Aronia arbutifolia (L.) Pers.

PHRAGMITES Adans. (Poaceae)

(*)P. australis (Cav.) Trin. ex Steud., Common Reed-grass

$=\mathrm{P}$. communis Trin.

Phragmites communis Trin.$=$ Phragmites australis (Cav.) Trin. ex Steud.

PHRYMA L. (Verbenaceae)

P. leptostachya L., Lopseed

$=$ P. leptostachya var. confertifolia Fern.

Phyllitis scolopendrium (L.) Newm. = Asplenium scolopendrium L. var. americanum (Fern.) Kartesz \& Gandhi

Phyllitis scolopendrium var. americanum Fern. = Asplenium scolopendrium L. var. americanum (Fern.) Kartesz \& Gandhi

PHYSALIS L. (Solanaceae)
*P. alkekengi L., Chinese Lantern, Japanese Lantern, Winter-cherry, Alkekengi, Strawberry Tomato

P. heterophylla Nees, Clammy Ground-cherry $=\mathrm{P}$. heterophylla var. ambigua (A. Gray) Rydb. of Zander \& Pierce 1979 $=\mathrm{P}$. heterophylla var. nyctaginea (Dunal) Rydb. $=\mathrm{P}$. viscosa of local authors)

P. longifolia Nutt., Long-leaf Ground-cherry $=$ P. longifolia var. subglabrata (Mackz. \& Bush) Cronq. $=$ P. subglabrata Mackz.\& Bush $=$ P. virginiana var. subglabrata $($ Mackz. \& Bush) Waterfall

P. pubescens L., Tall Hairy Ground-cherry, Strawberry Tomato, Dwarf Capegooseberry, Husk-tomato

var. integrifolia (Dunal) Waterfall $=\mathrm{P}$. pruinosa of NY reports, not $\mathrm{L}$.

Physalis subglabrata Mackz.\& Bush $=$ Physalis longifolia Nutt.

P. virginiana Mills., Virginia Ground-cherry $=$ P. longifolia (Nutt.) Trel. in part

Physalis virginiana var. subglabrata (Mackz. \& Bush) Waterfall $=$ Physalis longifolia Nutt.

Physalis viscosa (of authors) $=$ Physalis heterophylla Nees

Physalis viscosa L., excluded by Zander \& Pierce, 1979; no confirmed Ontario report (Morton \& Venn, 1990).

Physalodes physalodes (L.) Britt. $=$ Nicandra physalodes (L.) Gaertn.

PHYSOCARPUS Maxim. (Rosaceae)

P. opulifolius (L.) Maxim., Nine-bark

var. intermedius (Rydb.) Robins. Hairy-fruited Nine-bark

$=$ Opulaster intermedius Rydb. ex Britt.

var. opulifolius

$=$ Neillia opulifolia (L.) Brew.\& Wats.

$=$ Opulaster opulifolius (L.) Kuntze

$=$ Spiraea opulifolia L.

PHYSOSTEGIA Benth. (Lamiaceae)

P. virginiana (L.) Benth., False Dragon-head, Obedient Plant

$=$ Dracocephalum formosius (Lunnell) Rydb. $=$ D. speciosum Sweet 
$=\mathrm{D}$. virginianum $\mathrm{L}$.

$=$ Physostegia virginiana varieties

$=\mathrm{Ph}$. formosius Lunell

Physostegia formosius Lunell $=$ Physostegia virginiana (L.) Benth.

PHYTOLACCA L. (Phytolaccaceae)

P. americana L., Pokeweed, Poke, Pokeberry, Scoke, Pigeonberry

$=\mathrm{P}$. decandra L.

Phytolacca decandra L. = Phytolacca americana L.

PICEA Dietr. (Pinaceae)

Picea alba Link = Picea glauca $($ Moench $)$ Voss

Picea canadensis (Mill.) BSP. $=$ Picea glauca (Moench) Voss

[Picea glauca (Moench) Voss of Clinton's Journal (1864)]

$=$ Abies alba Michx.

$=$ Picea alba Link

= Picea canadensis (Mill.) BSP.

P. mariana (Mill) BSP., Black Spruce, Bog-spruce

$=$ Abies nigra Poir. of Clinton's Journal (1864)

$=\mathrm{P}$. nigra (Ait.) Link

Picea nigra (Ait.) Link = Picea mariana (Mill.)

BSB

PICRIS L. (Asteraceae)

*P. echioides L., Ox-tongue

*P. hieracioides L., Hawkweed Picris, Ox-tongue

PILEA Lindl. (Urticaceae)

P. fontana (Lunnell) Rydb., Clearweed

$=$ Adicia fontana Lunell

P. pumila (L.) A. Gray, Clearweed, Richweed, Coolwort

$=$ Adicia pumila (L.) Raf.

$=$ Pilea pumila var. deamii (Lunell) Fern.

Pilosella aurantiaca (L.) F. W. Schultz \& SchultzBip. = Hieracium aurantiacum L.

Pilosella caespitosa (Dumort.) P. D. Sell. \& C. West $=$ Hieracium caespitosum Dumort.

Pilosella officinarum F. W. Schultz \& Schultz-Bip. $=$ Hieracium pilosella $\mathrm{L}$.

Pilosella piloselloides (Vill.) Soják = Hieracium piloselloides Vill.

PIMPINELLA L. (Apiaceae)
Pimpinella integerrima (L.) Gray = Taenidia integerrima (L.) Drude

*P. saxifraga L., Burnet Saxifrage

PINGUICULA L. (Lentibulariaceae)

P. vulgaris L., Butterwort, Bog-violet

PINUS L. (Pinaceae)

*P. banksiana Lamb., Jack Pine

P. resinosa Soland., Red Pine, Norway Pine

P. strobus L., White Pine

*P. sylvestris L., Scotch Pine, Scots Pine

Piptatherum pungens (Torr. ex Spreng.) Dorn = Oryzopsis pungens (Torr. ex Spreng.) A. S. Hitchc.

Piptatherum racemosum Ricker ex A. S. Hitchc. = Oryzopsis racemosa (Sm.) Ricker ex A. S. Hitchc.

PISUM L. (Fabaceae)

Pisum maritimum L. = Lathyrus japonicus Willd. var. maritimus (L.) Kartesz \& Gandhi

*P. sativum L., Common, Garden-, Green or Field-pea

PLANTAGO L. (Plantaginaceae)

Plantago arenaria Waldst. \& Kit. = Plantago psyllium L.

*P. aristata Michx., Bracted Plantago, Rat-tail Plantain, Western Ripplegrass

P. cordata Lam., Heart-leaved Plantain

Plantago indica L., a nomen illeg. = Plantago psyllium L.

Plantago kamtschatica (of Day, 1882) $=$ Plantago rugelii Dcne.

*P. lanceolata L., English Plantain, Buck-horn Plantain, Rib-grass, Ripplegrass, Ribwort, Narrow-leaf Plantain

$=$ P. lanceolata var. sphaerostachya Mert. \& Koch

Plantago lanceolata var. sphaerostachya Mert. \& Koch $=$ Plantago lanceolata L .

*P. major L., Broad-leaved Plantain, Common Plantain, Door-yard Plantain, Car(t)-track Plant, White-man's Foot

$=$ P. major var. asiatica of authors, not (L.) Dcne. ex DC.

$=\mathrm{P}$. major var. intermedia (Gilib.) Dene. ex DC., not Pilger 
$=$ P. major var. pachyphila Pilger

*P. psyllium L., Flaxseed Plantain, Fleawort, Psyllium

$=$ P. arenaria Waldst. \& Kit.

$=$ P. indica L., a nomen illeg.

$=$ P. psyllium L., a nomen ambiguum

P. rugelii Dcne.,Rugel's Plantain, Pale Plantain, Grand Plantain, White-man's foot $=$ P. kamtschatica (of Day, 1882)

P. virginica L., Dwarf Plantain

PLATANTHERA L. Rich. (Orchidaceae)

Platanthera aquilonis Sheviak = Platanthera hyperborea (L.) Lindl.

P. blephariglottis (Willd.) Lindl., White Fringed Orchid

= Blephariglottis blephariglottis (Willd.) Rydb. ex Britt.

$=$ Habenaria blephariglottis (Willd.) Hook.

$=$ Habenaria blephariglottis var. holopetala (Lindl.) A. Gray

Platanthera bracteata of Clinton's collecting journal = Coeloglossum viride (L.) Hartm.

P. clavellata (Michx.) Luer, Green Woodland Orchid

= Gymnadeniopsis clavellata (Michx.) Rydb. ex Britt.

$=$ Habenaria clavellata (Michx.) Spreng.

P. dilatata (Pursh) Lindl. ex Beck, Tall Leafy

White Orchid, Bog-candle

$=$ Habenaria dilatata (Pursh) Hook.

$=\mathrm{H}$. dilatata var. media (Rydb.) Ames

= Limnorchis dilatata (Pursh) Rydb. ex Britt.

$=$ L. dilatata var. linearifolius Rybd.

P. flava (L.) Lindl., Grass-green Orchid

var. herbiola (R. Br.) Luer

$=$ Habenaria flava (L.) R. Br. var. herbiola

(R. Br.) Ames \& Correll

$=\mathrm{H}$. flava var. virescens (Muhl. ex Willd.) Fern.

$=\mathrm{H}$. herbiola R. Br.

$=\mathrm{H}$. virescens Muhl. ex Willd.

$=$ Perularia flava (L.) Farw. of NY reports

$=$ Tulotis herbiola (R. Br.) Raf.

P. grandiflora (Bigel.) Lindl., Large Purplefringed Orchid

= Blephariglottis grandiflora (Bigel.) Rydb. ex Britt.
$=$ Habenaria fimbriata (Dryand. ex Ait.) R. $\mathrm{Br}$.

$=\mathrm{H}$. peramoena of some NY reports, not $\mathrm{A}$. Gray (see P. peramoena below)

$=\mathrm{H}$. psycodes var. grandiflora (Bigel.) A. Gray

P. hookeri (Torr. ex A. Gray) Lindl., Hooker's Orchid

$=$ Habenaria hookeri Torr. ex A. Gray

= Lysias hookeri (Torr. ex A. Gray) Rydb. ex Britt.

$=\mathrm{L}$. hookeri of authors

P. hyperborea (L.) Lindl., Northern Leafy Green Orchid

$=$ Habenaria hyperborea (L.) R. Br.

= Limnorchis hyperborea (L.) Rydb. ex Britt.

$=$ Platanthera aquilonis Sheviak $[=\mathrm{P}$. hyberborea of most N. American reports, not (L.) Lindl., sec NYFA July 2005]]

P. lacera (Michx.) G. Don, Ragged Fringed Orchid

= Blephariglottis lacera (Michx.) Farw.

$=$ Habenaria lacera (Michx.) R. Br.

P. leucophaea (Nutt.) Lindl., Eastern Prairie Fringed Orchid

$=$ Blephariglottis leucophaea (Nutt.) Farw.

$=$ Habenaria leucophaea (Nutt.) A. Gray

P. macrophylla (Goldie) P. M. Brown, Large Round-leaf Orchid, Pad-leaf

$=$ Habenaria macrophylla Goldie

$=$ Lysias macrophylla (Goldie) House

$=$ Platanthera orbiculata var. macrophylla (Goldie) Luer

P. orbiculata (Pursh) Lindl., Round-leaved Orchid = Habenaria orbiculata (Pursh) Torr.

$=$ Lysias orbiculata (Pursh) Rydb. ex Britt.

[Platanthera peramoena A. Gray, excluded: original report due to an error of transcription by Day (1882), and corrected in that

publication (see Zenkert, 1934, p. 131).

Mitchell and Tucker (1997) indicate NY

reports of P. peramoena are either

Platanthera grandiflora (Bigel.) Lindl. or, less often, P. psycodes (L.) Lindl.]

P. psycodes (L.) Lindl., Small Purple-fringed Orchid

$=$ Blephariglottis psycodes (L.) Rydb. ex Britt. 
= Habenaria peramoena of some NY reports, not A. Gray

$=$ H. psycodes (L.) Spreng.

PLATANUS L. (Platanaceae)

P. occidentalis L., Sycamore, Plane-tree, Buttonwood

POA L. (Poaceae)

P. alsodes A. Gray, Grove Meadow-grass, Speargrass, Woodland Bluegrass

= Poa paludigena of Ontario reports (Morton \& Venn 1990).

*P. annua L., Spear-grass, Annual Bluegrass

*P. chapmaniana Scribn., Chapman's Blue-grass

*P. compressa L., Canada Blue-grass, Wiregrass

Poa debilis Torr., not Thuill. $=$ Poa languida Hitchc.

P. languida Hitchc., Weak Spear-grass

$=\mathrm{P}$. debilis Torr.

= P. saltuensis Fern. \& Wieg. ssp. languida

(A. S. Hitchc.) A. Haines

P. nemoralis L., Wood Bluegrass

Poa paludigena of Ontario reports = Poa alsodes A. Gray (Morton \& Venn 1990).

P. palustris L., Fowl Meadow-grass, Fowl Bluegrass

$=$ P. serotina Ehrh

*P. pratensis L., Kentucky Bluegrass, June-grass $=\mathrm{P}$. angustifolia $\mathrm{L}$.

$=$ P. pratensis ssp. angustifolia (L.) Gaudin

P. saltuensis Fern. \& Wieg., Bushy Pasture Speargrass, Old-pasture Bluegrass

Poa saltuensis Fern. \& Wieg. ssp. languida (A. S. Hitchc.) A. Haines = Poa languida Hitchc.

Poa serotina Ehrh. = Poa palustris L.

P. sylvestris A. Gray, Sylvan Spear-grass, Woodland Bluegrass

*P. trivialis L., Rough-stalked Meadow-grass, Rough Bluegrass

PODOPHYLLUM L. (Berberidaceae)

P. peltatum L., May-apple, Wild Mandrake

POGONIA Juss. (Orchidaceae)

P. ophioglossoides (L.) Juss., Rose Pogonia, Snake's Mouth

Pogonia pendula (Nutt.) Lindl. $=$ Triphora trianthophora (Sw.) Rydb.
Pogonia trianthophora $(\mathrm{Sw}$.$) BSP. =$ Triphora trianthophora (Sw.) Rydb.

Pogonia verticillata (Muhl. ex Willd.) Nutt. = Isotria verticillata (Muhl. ex Willd.) Raf.

Poinsettia dentata (Michx.) Klotzsch \& Garcke = Euphorbia dentata Michx.

POLANISIA Raf. (Capparidaceae)

P. dodecandra (L.) DC., Clammyweed

$=$ Jacksonia trifoliata Raf.

$=$ Polanisia graveolens Raf.

$=\mathrm{P}$. dodecandra ssp. trachysperma of NY reports, not (Torr. \& A. Gray) Iltis

$=$ P. trachysperma of NY reports, not Torr. \& A. Gray

Polanisia graveolens Raf. $=$ Polanisia dodecandra (L.) DC.

Polanisia trachysperma of NY reports, not Torr. \& A. Gray = Polanisia dodecandra (L.) DC.

POLEMONIUM L. (Polemoniaceae)

Polemonium coeruleum Gray, not L. = Polemonium van-bruntiae Britt.

Polemonium caeruleum ssp. vanbruntiae (Britt.) Davidson $=$ Polemonium van-bruntiae Britt.

P. reptans L., Greek Valerian, Polemony, Jacob's Ladder

P. van-bruntiae Britt., American Jacob's Ladder, Greek Valerian, Charity

$=$ P. caeruleum ssp. vanbruntiae (Britt.)

Davidson

$=\mathrm{P}$. coeruleum Gray, not $\mathrm{L}$.

POLYGALA L. (Polygalaceae)

Polygala ambigua Nutt. = Polygala verticillata L. var. ambigua (Nutt.) Wood

P. incarnata L., Pink Milkwort

P. paucifolia Wild., Fringed Polygala, Gay-wings, Flowering Wintergreen, Bird-on-the-Wing

P. polygama Walt., Racemed Milkwort, Bitter Milkwort

$=\mathrm{P}$. polygama var. obtusata Chodat

Polygala pretzii Pennell $=$ Polygala verticillata $\mathrm{L}$. var. verticillata

*P. sanguinea L., Purple Milkwort, Rose Milkwort, Field-milkwort $=\mathrm{P}$. viridescens $\mathrm{L}$.

P. senega L., Seneca Snakeroot, Mountain-flax 
= P. senega var. latifolia Torr. \& A. Gray

P. verticillata L., Whorled Milkwort

var. verticillata

$=\mathrm{P}$. pretzii Pennell

Polygala viridescens L. = Polygala sanguinea L.

POLYGONATUM Mill. (Liliaceae)

P. biflorum (Walt.) Ell., Small Solomon's-seal

= P. canaliculatum of authors, not (Muhl.) Pursh

Polygonatum biflorum var. commutatum (Schultes $\&$ Schultes) Morong = Polygonatum commutatum (Schultes \& Schultes) A.

Dietr.

Polygonatum biflorum var. hebetifolium Gates = Polygonatum commutatum (Schultes \& Schultes) A. Dietr.

Polygonatum canaliculatum of authors, not (Muhl.) Pursh $=$ Polygonatum biflorum (Walt.) Ell.

P. commutatum (Schultes \& Schultes) A. Dietr., Great Solomon's Seal

$=\mathrm{P}$. biflorum var. commutatum (Schultes $\&$ Schultes) Morong

$=\mathrm{P}$. biflorum var. hebetifolium Gates

$=\mathrm{P}$. giganteum Dietr.

Polygonatum giganteum A. Dietr. = Polygonatum commutatum (Schultes \& Schultes) A. Dietr.

P. pubescens (Willd.) Pursh, Downy Solomon'sseal

POLYGONUM L. (Polygonaceae)

(*?) P. achoreum Blake, Homeless Knotweed

Polygonum acre HBK = Polygonum punctatum

Ell. var. punctatum

P. amphibium L., Swamp-smartweed, Watersmartweed, Amphibious Smartweed

$=$ Persicaria amphibia (L.) S. Gray

var. emersum Michx.

$=\mathrm{P}$. coccineum Muhl. ex Willd.

$=\mathrm{P}$. coccineum var. terrestre Willd.

$=\mathrm{P}$. inundatum Raf.

= P. muhlenbergii S. Wats.

P. amphibium L., Water-smartweed, Amphibious Smartweed

var. emersum Michx., Swamp-smartweed $=$ P. amphibium var. terrestre A. Gray, not Leers, nor Leyss.

$=\mathrm{P}$. coccineum Muhl. ex Willd. var. emersum $\times$ stipulaceum

$=\mathrm{P}$. hartwrightii A. Gray

var. stipulaceum Colem. [not (Colem.) Fern.], Floating Smartweed

$=$ P. fluitans Eat.

$=$ P. natans Eat.

$=\mathrm{P}$. amphibium var. amphibium of North

American reports, not L.

$=\mathrm{P}$. amphibium var. aquaticum Torr.

$=\mathrm{P}$. amphibium var. natans Michx.

Polygonum amphibium var. amphibium of North American reports, not L. $=$ Polygonum amphibium var. stipulaceum Colem.

Polygonum amphibium var. natans Michx. = Polygonum var. stipulaceum Colem. (not (Colem.) Fern.)

Polygonum amphibium var. terrestre A. Gray, not Leers, nor Leyss. $=$ Polygonum amphibium var. emersum Michx.

Polygonum arenastrum Jord. ex Boreau $=$ Polygonum aviculare L. ssp. depressum (Meisner) Arcangeli

P. arifolium L., Halberd-leaved Tear-thumb = Persicaria arifolia (L.) Haraldson

$=$ Polygonum arifolium var. pubescens (Keller) Fern.

$=$ Tracaulon arifolium (L.) Small

Polygonum atlanticum (B. Robinson) Bickn. = Polygonum ramosissimum Michx. var. prolificum Small

P. aviculare L., Common Knotweed

ssp. aviculare

$=$ P. aviculare var. arenastrum (Bor.) Rouy

$=\mathrm{P}$. aviculare var. vegetum Ledeb.

$=\mathrm{P}$. heterophyllum Lindm.

$=\mathrm{P}$. monspeliensis Pers.

$=\mathrm{P}$. neglectum Besser

*ssp. depressum (Meisner) Arcangeli

$=\mathrm{P}$. arenastrum Jord. ex Boreau

Polygonum aviculare var. agrarium K. Koch = Polygonum erectum L.

Polygonum aviculare ssp. buxiforme (Small) Costea \&Tardif = Polygonum buxiforme Small

Polygonum aviculare var. crassifolium Lange $=$ Polygonum buxiforme Small

Polygonum aviculare var. erectum (L.) Roth $=$ Polygonum erectum L. 
Polygonum aviculare var. laterale Michx. =

Polygonum erectum L.

Polygonum aviculare var. littorale (Link) Mert. = Polygonum buxiforme Small

Polygonum bicorne Raf. $=$ Polygonum pensylvanicum $\mathrm{L}$.

P. buxiforme Small, Knotweed, Knotgrass

$=\mathrm{P}$. aviculare ssp. buxiforme (Small) Costea \&Tardif

$=\mathrm{P}$. aviculare var. crassifolium Lange

$=\mathrm{P}$. aviculare var. littorale (Link) Mert.

$=\mathrm{P}$. fowleri of $\mathrm{NY}$ reports, not $\mathrm{B}$. Robinson

$=\mathrm{P}$. littorale $($ Link) Koch

*P. cespitosum Blume

*var. longisetum (DeBruyn) Stewart, Low

Smartweed

$=\mathrm{P}$. longisetum DeBruyn

$=\mathrm{P}$. posumbo Hamilt.

P. cilinode Michx., Fringed Bindweed, False Buckwheat

$=$ Bilderdykia cilinode (Michx.) Greene

$=$ Fallopia cilinodis (Michx.) Holub.

Polygonum coccineum Muhl. ex Willd. =

Polygonum amphibium L. var. emersum

Michx.

Polygonum coccineum var. terrestre Willd. =

Polygonum amphibium L. var. emersum

Michx.

*P. convolvulus L., Black Bindweed, Corn

Bindweed

$=$ Bilderdykia convolvulus (L.) Dumort.

$=$ Fallopia convolvulus (L.) Á. . Löve

Polygonum cristatum Engelm. \& Gray =

Polygonum scandens L. var. cristatum

Engelm. \& A. Gray

*P. cuspidatum Sieb.\& Zucc., Japanese

Knotweed, Japanese Bamboo

$=$ Fallopia japonica (Hout.) Dene ssp.

japonica

$=$ Polygonum zuccarinii Small

= Reynoutria japonica Houtt.

Polygonum dubium Stein $=$ Polygonum persicaria L.

Polygonum dumetorum L. = Polygonum scandens var. dumetorum (L.) Gleason

P. erectum L., Erect Knotweed

$=\mathrm{P}$. aviculare var. agrarium K. Koch

$=$ P. aviculare var. erectum (L.) Roth

$=\mathrm{P}$. aviculare var. laterale Michx.
Polygonum exsertum Small, in large part = Polygonum ramosissimum Michx. var. prolificum Small

Polygonum fluitans Eat. $=$ Polygonum amphibium L. var. stipulaceum Colem.

Polygonum fowleri of NY reports, not B. Robinson $=$ Polygonum buxiforme Small

Polygonum hartwrightii A. Gray = Polygonum amphibium L. var. emersum $\times$ stipulaceum

Polygonum heterophyllum Lindm. $=$ Polygonum aviculare $\mathrm{L}$.

*P. hydropiper L., Water-smartweed, Waterpepper

$=$ Persicaria hydropiper (L.) Opiz.

P. hydropiperoides Michx., Mild Watersmartweed, Water-pepper

var. hydropiperoides

$=$ Persicaria hydropiperoides (Michx.) Small

= Polygonum hydropiperoides var. strigosum Small

$=$ P. mite Pers., not Schrank sec. House (1924).

Polygonum hydropiperoides var. setaceum (Baldw. ex Ell.) Gleason $=$ Polygonum setaceum Baldw. ex Ell. var. interjectum Fern.

Polygonum incanum F. Schmidt $=$ Polygonum pensylvanicum $\mathrm{L}$.

Polygonum incarnatum Ell. = Polygonum lapathifolium L.

Polygonum inundatum Raf. $=$ Polygonum amphibium L. var. emersum Michx.

P. lapathifolium L., Dock-leaved Smartweed

$=$ Persicaria lapathifolia (L.) Small [(L.) S. F. Gray sec. NYFA 2005]

$=$ Polygonum lapathifolium var. incanum (Schmidt) Koch

= P. lapathifolium var. nodosum (Pers.) Weinm.

$=$ P. lapathifolium var. punctatum Beck ex Reichenb.

$=$ P. lapathifolium var. salicifolium Sibth.

$=\mathrm{P}$. incarnatum Ell.

$=$ P. nodosum Pers.

$=$ P. scabrum Moench

$=\mathrm{P}$. tomentosum A. Gray

Polygonum littorale $($ Link) Koch $=$ Polygonum buxiforme Small

Polygonum longistylum Small = Polygonum pensylvanicum $\mathrm{L}$. 
Polygonum minus Huds. $=$ Polygonum persicaria L.

Polygonum mite of American authors, not Schrank, also P. mite Gilib. sec. House $(1924)$ = Polygonum persicaria L.

Polygonum mite Pers., not Schrank, sec. House $(1924)=$ P. hydropiperoides Michx. var. hydropiperoides

Polygonum monspeliensis Pers. = Polygonum aviculare L.

Polygonum muhlenbergii S. Wats. = Polygonum amphibium L. var. emersum Michx.

Polygonum natans Eat. $=$ Polygonum amphibium L. var. stipulaceum Colem.

Polygonum neglectum Besser $=$ Polygonum aviculare L.

Polygonum nodosum Pers. $=$ Polygonum lapathifolium L.

*P. orientale L., Prince's Feather, Kiss-me-overthe-Garden-Gate

$=$ Persicaria orientalis (L.) Spach

P. pensylvanicum L., Pennsylvania Smartweed

$=$ Persicaria pensylvanica (L.) Small

$=$ Polygonum bicorne Raf.

$=\mathrm{P}$. incanum F. Schmidt

$=\mathrm{P}$. longistylum Small

$=\mathrm{P}$. pensylvanicum var. eglandulosum $\mathrm{J}$. Meyers

$=\mathrm{P}$. pensylvanicum var. laevigatum Fern.

*P. persicaria L., Ladies-thumb

$=$ Persicaria persicaria (L.) Small

$=$ P. maculosa S. F. Gray

$=$ Polygonum dubium Stein

$=$ P. minus Huds.

$=$ P. mite of American authors, not Schrank

$=\mathrm{P}$. puritanorum Fern.

Polygonum posumbo Hamilt. $=$ Polygonum cespitosum Blume var. longisetum (DeBruyn) Stewart

Polygonum prolificum (Small) Robins. = Polygonum ramosissimum Michx. var. prolificum Small

P. punctatum Ell., Dotted Smartweed var. confertiflorum (Meisn.) Fassett $=\mathrm{P}$. punctatum var. leptostachyum (Meisn.) Small

$=$ P. punctatum var. parvum Vict. \& Rouss. var. punctatum

$=$ Persicaria punctata (Ell.) Small
$=$ Polygonum acre Kunth

Polygonum punctatum var. majus (Meisn. ex DC.)

Fassett $=$ Polygonum robustius $($ Small $)$

Fern.

Polygonum puritanorum Fern.$=$ Polygonum persicaria L.

*P. ramosissimum Michx., Bushy Knotweed

*var. prolificum Small, Saltmarsh-knotweed $=\mathrm{P}$. atlanticum (B. Robinson) Bickn.

$=$ P. exsertum Small, in large part

$=\mathrm{P}$. prolificum (Small) B. Robinson

*var. ramosissimum

$=$ P. allocarpum Blake

$=$ P. exsertum Small in part

P. robustius (Small) Fern., Robust Smartweed, Large Water-smartweed

$=$ Persicaria robustior $($ Small) Bickn.

$=$ Polygonum punctatum var. majus (Meisn. ex DC.) Fassett

$=\mathrm{P}$. punctatum var. robustior Small

*P. sachalinense F. Schmidt ex Maxim., Giant Knotweed

$=$ Fallopia sachalinensis $($ Schmidt ex Maxim.) Dcne.

$=$ Reynoutria sachalinensis (Schmidt ex Maxim.) Nakai ex Mori

P. sagittatum L., Arrow-leaved Tear-thumb, Arrow-vine, Scratch-grass

$=$ Persicaria sagittata (L.) H. Gross

$=$ Tracaulon sagittatum (L.) Small

Polygonum scabrum Moench $=$ Polygonum lapathifolium L.

P. scandens L., Climbing False Buckwheat

var. cristatum (Engelm. \& A. Gray) Gleason, Crested False Buckwheat

$=$ Fallopia scandens (L.) Holub. var. cristatum (Engelm. \& A. Gray) H. R. Hinds

$=$ Polygonum cristatum Engelm. \& A. Gray

*var. dumetorum (L.) Gleason, Thicket False Buckwheat

$=$ Fallopia dumetorum (L.) Holub.

$=$ Polygonum dumetorum L.

var. scandens

$=$ Fallopia scandens (L.) Holub. var. scandens

P. setaceum Baldw. ex Ell., Dock-leaved

Smartweed

var. interjectum Fern.

$=$ Persicaria setacea (Baldw. ex Ell.) Small 
$=\mathrm{P}$. hydropiperoides var. setaceum (Baldw. ex Ell.) Gleason in part

$=\mathrm{P}$. setaceum var. tonsum Fern.

[Polygonum tenue Michx., Slender Knotweed of possible occ. in Regl. M. Niag. Oldham 1999.]

Polygonum tomentosum A. Gray = Polygonum lapathifolium L.

P. virginanum L., Virginia Knotweed

$=$ Persicaria virginiana (L.) Gaert.

$=$ Tovara virginiana $(\mathrm{L}$.$) Raf.$

Polygonum zuccarinii Small $=$ Polygonum cuspidatum Sieb.\& Zucc.

POLYMNIA L. (Asteraceae)

P. canadensis L., Small-flowered Leaf-cup = Osteospermum canadense (L.) House

P. uvedalia (L.) L., Large-Flowered Leaf-cup $=$ Osteospermum uvedalia L.

$=$ Smallanthus uvedalia (L.) Mackz. ex Small

POLYPODIUM L. (Polypodiaceae)

Polypodium bulbiferum L. $=$ Cystopteris bulbifera (L.) Bernh.

Polypodium carthusianum Vill. $=$ Dryopteris carthusiana (Vill.) Fuchs

Polypodium connectile Michx. $=$ Phegopteris connectilis (Michx.) Watt

Polypodium cristatum L. $=$ Dryopteris cristata (L.) A. Gray

Polypodium dryopteris L. = Gymnocarpium dryopteris (L.) Newm.

Polypodium fragile L. = Cystopteris fragilis (L.) Bernh.

Polypodium hexagonopterum Michx. = Phegopteris hexagonoptera (Michx.) Fée,

Polypodium phegopteris L. $=$ Phegopteris connectilis (Michx.) Watt

P. virginianum L., Common Polypody, Rock Tripe

$=\mathrm{P}$. vulgare of $\mathrm{NY}$ Reports, not L.

$=\mathrm{P}$. vulgare var. americanum Hooker

$=\mathrm{P}$. vulgare var. virginianum (L.) Eat.

P. appalachianum Haufler \& Windham, Appalachian Rockcap Fern.

$=\mathrm{P}$. virginianum L. of NY reports in part
POLYSTICHUM Roth ex Roemer

(Polypodiaceae)

P. acrostichoides (Michx.) Schott, Christmas-fern, Shield-fern

$=$ Aspidium acrostichoides Sw.

= A. acrostichoides var. incisum A. Gray

= Nephrodium acrostichoides Michx.

P. lonchitis (L.) Roth, Northern Holly-fern

PONTEDERIA L. (Pontederiaceae)

P. cordata L., Pickerel-weed $=\mathrm{P}$. cordata var. lancifolia (Muhl.) Torr.

$=$ P. lanceolata Nutt.

Pontederia lanceolata Nutt. $=$ Pontederia cordata L .

POPULUS L. (Salicaceae)

*P. alba L., White Poplar, Silver-leaf Poplar $=$ P. major Mill.

$=\mathrm{P}$. nivea Willd.

Populus angulata Ait. $=$ Populus deltoides Bartr. ex Marsh.

P. balsamifera L., Balsam Poplar

= P. balsamifera var. lanceolata Marsh.

= P. balsamifera var. subcordata Hyl.

$=\mathrm{P}$. carolinensis Moench

Populus balsamifera $\times$ deltoides $=P . \times$ jackii

Sarg., Balm of Gilead

$=\mathrm{P}$. candicans of authors, not Ait.

$=$ P. $\times$ gileadensis Roul .

Populus balsamifera var. subcordata Hyl. = Populus balsamifera L.

Populus candicans Ait. $=[$ Populus balsamifera $\times$ deltoides $=$ P. $\times$ jackii Sarg.]

Populus carolinensis Moench $=$ Populus balsamifera L.

P. deltoides Bartr. ex Marsh., Cottonwood $=\mathrm{P}$. angulata Ait. $=$ P. monilifera Ait.

$(*) \mathbf{P}$. deltoides $\times$ nigra $=\mathbf{P} . \times$ canadensis Moench, Carolina Poplar $=$ P. $\times$ eugenei Simon-Louis

Populus dilatata Ait. $=$ Populus nigra L.

Populus $\times$ eugenei Simon-Louis $=[$ Populus deltoides $\times$ nigra $=$ P. $\times$ canadensis Moench]

Populus $\times$ gileadensis Rouleau $=[$ Populus balsamifera $\times$ deltoides $=$ P. $\times$ jackii Sarg.]

P. grandidentata Michx., Large-toothed Aspen, Big-Tooth Aspen 
$=$ P. trepida Willd.

Populus italica (DuRoi) Moench $=$ Populus nigra L.

Populus major Mill. = Populus alba L.

Populus monilifera Ait. = Populus deltoides Bartr. ex Marsh.

*P. nigra L., Black Poplar, Lombardy Poplar $=$ P. dilatata Ait.

= P. italica (DuRoi) Moench

$=$ P. nigra var. italica DuRoi

$=\mathrm{P}$. pyramidata Moench

Populus nigra var. italica Moench $=$ Populus nigra L.

Populus nivea Willd. $=$ Populus alba L.

Populus pyramidata Moench $=$ Populus nigra L .

P. tremuloides Michx., Quaking or Trembling Aspen

= P. tremula L. ssp. tremuloides (Michx.) Á. \& D. Löve

Populus tremula L. ssp. tremuloides (Michx.) Á. \& D. Löve $=$ Populus tremuloides Michx.

Populus trepida Willd. $=$ Populus grandidentata Michx.

PORTERANTHUS Britt. ex Small (Rosaceae)

P. trifoliatus (L.) Britt., Bowman's-root, Indian Physic

$=$ Gillenia trifoliata (L.) Moench

$=$ Spiraea trifoliata $\mathrm{L}$.

PORTULACA L. (Portulacaceae)

*P. oleracea L., Common Purslane, Pussley $=\mathrm{P}$. oleracea ssp. sativa (Haw) Célak

POTAMOGETON L. (Potamogetonaceae)

P. alpinus Balbis, Alpine Pondweed

$=\mathrm{P}$. alpinus var. subellipticus (Fern.) Ogden

$=$ P. alpinus var. tenuifolius (Raf.) Ogden

$=\mathrm{P}$. rufescens of Canadian authors, not of

Schrader

$=\mathrm{P}$. tenuifolius Raf.

Potamogeton americanus var. noveboracensis

(Morong) Benn. $=$ Potamogeton nodosus

Poir.

P. ampifolius Tuckerm., Broad-leaved Pondweed Potamogeton angustifolius of authors, not Berch.

$\&$ Presl. $=$ Potamogeton illinoensis

Morong
Potamogeton berchtoldii Fieber $=$ Potamogeton pusillus L. var. tenuissimus Mert. \& Koch

Potamogeton bupleuroides L. $=$ Potamogeton perfoliatus L.

Potamogeton capillaceus Poir., in part $=$ Potamogeton diversifolius Raf.

Potamogeton claytoni Tuckerman $=$ Potamogeton epihydrus Raf.

Potamogeton compressus L. $=$ Potamogeton zosteriformis Fern.

*P. crispus L., Curly Pondweed, Curly Muckweed

Potamogeton dimorphus Raf. $=$ Potamogeton spirillus Tuckerm.

P. diversifolius Raf., Rafinesque's Pondweed $=$ P. capillaceus Poir., in part

$=\mathrm{P}$. hybridus (of authors)

P. epihydrus Raf., Nuttall's Pondweed $=\mathrm{P}$. claytoni Tuckerman

$=$ P. epihydrus var. cayugensis (Weig.) Benn.

$=$ P. epihydrus var. nuttallii (Cham. \& Schlect.) Fern.

$=\mathrm{P}$. epihydrus var. ramosus (Peck) House

Potamogeton filiformis var. borealis (Raf.) St. John $=$ Coleogeton filiformis (Pers.) D. Les \& R. Haynes ssp. alpinus (Blytt) D. Les \& R. Haynes

Potamogeton filiformis var. macounii Morong = Coleogeton filiformis (Pers.) D. Les \& R. Haynes ssp. alpinus (Blytt) D. Les \& R. Haynes

Potamogeton filiformis var. occidentalis (Robbins.) Morong $=$ Coleogeton filiformis (Pers.) D. Les \& R. Haynes ssp. occidentalis (Robbins) D. Les \& R. Haynes

P. foliosus Raf., Leafy Pondweed $=\mathrm{P}$. niagarensis Tuckerm.

$=\mathrm{P}$. foliosus var. genuinus Fern.

$=$ P. foliosus var macellus Fern.

$=$ P. pauciflorus (of authors)

P. friesii Rupr., Fries' Pondweed

P. gramineus L., Grassy Pondweed var. gramineus

$=\mathrm{P}$. heterophyllus auct. non Schreber

$=\mathrm{P}$. gramineus var. graminifolius Fries

$=\mathrm{P}$. gramineus var. maximus Morong ex Benn.

$=\mathrm{P}$. gramineus var. myriophyllus Robbins

$\mathbf{P}$. gramineus $\times$ illinoensis $=\mathbf{P} . \times$ spathuliformis (Robbins) Morong, Pondweed 
Potamogeton heterophyllus (of authors) = Potamogeton gramineus L.

Potamogeton hybridus (of authors) $=$ Potamogeton diversifolius Raf.

P. illinoensis Morong, Illinois Pondweed

$=\mathrm{P}$. angustifolius of authors, not Berch. $\&$ Presl.

$=\mathrm{P}$. lucens (of American authors), not L.

Potamogeton interior Rydb. = Coleogeton

filiformis (Pers.) D. Les \& R. Haynes ssp.

occidentalis (Robbins) D. Les \& R.

Haynes

Potamogeton lateralis Morong in part $=$

Potamogeton vaseyi Robbins

Potamogeton lateralis Morong in part $=$

Potamogeton pusillus L. var. tenuissimus

Mert. \& Koch

Potamogeton lonchites Tuckerm. $=$ Potamogeton nodosus Poir.

Potamogeton lonchites var. noveboracensis Morong $=$ Potamogeton nodosus Poir .

Potamogeton $\times$ longiligulatus Fern.$=$ Potamogeton strictifolius Benn.

Potamogeton lucens of American authors, not L. = Potamogeton illinoensis Morong

P. natans L., Floating Pondweed

Potamogeton niagarensis Tuckerm. $=$ Potamogeton foliosus Raf.

P. nodosus Poir., Knotty Pondweed

$=\mathrm{P}$. americanus var. noveboracensis

(Morong) Benn.

$=\mathrm{P}$. lonchites Tuckerm.

$=\mathrm{P}$. lonchites var. noveboracensis Morong

P. obtusifolius Mert. \& Koch, Blunt-leaved Pondweed

Potamogeton panormitanus Biv. $=$ Potamogeton pusillus L.

Potamogeton pauciflorus (of authors) $=$ Potamogeton foliosus Raf.

Potamogeton pectinatus L. $=$ Coleogeton pectinatum (L.) D. Les \& R. Haynes

P. perfoliatus L., Clasping-leaved Pondweed, White-stemmed Pondweed

$=\mathrm{P}$. bupleuroides Fern.

$=$ P. perfoliatus var. bupleuroides (Fern.) Farw.

P. praelongus Wulfen, White-stemmed Pondweed

P. pusillus L., Small Pondweed var. pusillus
$=\mathrm{P}$. panormitanus Biv.

var. tenuissimus Mert. \& Koch

$=\mathrm{P}$. berchtoldii Fieber

$=\mathrm{P}$. lateralis Morong in part

Potamogeton pusillus var. pseudorutilus Benn. = Potamogeton strictifolius Benn.

P. richardsonii (Benn.) Rydb., Richardson's

Pondweed, Red-head Pondweed

P. robbinsii Oakes, Robbins' Pondweed

Potamogeton rufescens Schrad. $=$ Potamogeton alpinus Balbis

Potamogeton rutilis of authors, not Wulf. ex R. \& S.

P. spirillus Tuckerm., Coiled Pondweed $=$ P. dimorphus Raf.

P. strictifolius Benn., Straight-leaved Pondweed $=\times$ longiligulatus Fern.

$=\mathrm{P}$. pusillus var. pseudorutilus Benn.

$=$ P. rutilis of authors, not Wulf. ex R. \& S.

$=$ P. strictifolius var. rutiloides Fern.

$=$ P. strictifolius var. typicus Fern.

Potamogeton tenuifolius Raf. $=$ Potamogeton alpinus Balbis

Potamogeton vaginatus of NY reports, not Turcz. $=$ Coleogeton filiformis (Pers.) D. Les \& R. Haynes ssp. occidentalis (Robbins) D. Les \& R. Haynes

P. vaseyi Robbins, Vasey's Pondweed $=\mathrm{P}$. lateralis Morong in part

P. zosteriformis Fern., Flat-stem Pondweed $=\mathrm{P}$. compressus $\mathrm{L}$.

POTENTILLA L. (Rosaceae)

P. anserina L., Silverweed, Goose-grass, Goosetansy

ssp. anserina

$=$ Argentina anserina (L.) Rydb.

$=$ A. babcockiana Rydb.

= Potentilla anserina var. sericea Hayne

P. argentea L., Silvery Cinquefoil, Hoary

Cinquefoil

P. arguta Pursh, Tall Cinquefoil

$=$ Drymocallis agrimonioides (Pursh) Rydb.

$=$ Geum agrimonioides Pursh

$=$ Potentilla confertiflora Torrey

P. canadensis L., Dwarf Cinquefoil, Five-fingers

$=$ P. canadensis var. villosissima Fern.

$=\mathrm{P}$. caroliniana of authors, not Poir.

$=\mathrm{P}$. pumila Poir. ex Lam. 
= P. simplex sensu House (1924), not Michx.

Potentilla caroliniana of authors, not Poir. $=$ Potentilla canadensis L.

Potentilla comarum Nestler $=$ Potentilla palustris (L.) Scop.

Potentilla confertiflora Torrey $=$ Potentilla arguta Pursh

Potentilla floribunda Pursh $=$ Potentilla fruticosa ssp. floribunda (Pursh) Elkington

P. fruticosa L., Shubby Cinquefoil ssp. floribunda (Pursh) Elkington $=$ Dasiphora fruticosa auct. non (L.) Rydb.

$=$ D. floribunda (Pursh) Kartesz

= Pentaphylloides floribunda (Pursh) Á. Löve

$=$ Potentilla floribunda Pursh

$=\mathrm{P}$. fruticosa var. tenuifolia Lehm.

P. norvegica L., Rough Cinquefoil ssp. monspeliensis (L.) Aschers. \& Graeb.

$=\mathrm{P}$. norvegica var. hirsuta (Michx) Torr.\& A. Gray

Potentilla obscura Nestler $=$ Potentilla recta L .

P. palustris (L.) Scop., Marsh-cinquefoil, Fivefingers, Purple Cinquefoil

$=$ Comarum palustre $\mathrm{L}$.

$=\mathrm{C}$. palustre var. villosum Pers.

$=$ Potentilla comarum Nestler

$=$ P. palustris var. villosa (Pers.) Lehm.

P. paradoxa Nutt. ex Torr. \& A. Gray, Bushy Cinquefoil

$=\mathrm{P}$. supina Michx.

Potentilla pumila Poir. ex Lam. $=$ Potentilla canadensis $\mathrm{L}$.

*P. recta L., Sulphury Cinquefoil, Five-fingers $=$ P. obscura Nestler

$=\mathrm{P}$. recta var. obscura (Neatler) Koch

$=$ P. recta var. sulphurea (Lam. \& DC.) Peyr

= P. sulphurea Lam. \& DC.

*P. reptans L., Creeping Cinquefoil

P. simplex Michx., Common Cinquefoil, Old-field Cinquefoil

$=\mathrm{P}$. canadensis of $\mathrm{NY}$ reports, not $\mathrm{L}$.

$=$ P. simplex var. calvescens Fern.

Potentilla simplex sensu House (1924), not Michx. $=$ Potentilla canadensis L.

Potentilla sulphurea Lam. \& DC. $=$ Potentilla recta L.

Potentilla supina Michx. $=$ Potentilla paradoxa Nutt.
Poterium canadense (L.) Gray $=$ Sanguisorba canadensis L.

Poterium sanguisorba of authors, not L. = Sanguisorba minor Scop.

PRENANTHES L. (Asteraceae)

P. alba L., Lion's-foot, White Lettuce, Rattlesnake-root

$=$ Nabalus albus (L.) Hook.

P. altissima L., Tall Lion's-foot, Rattlesnake-root, White Lettuce

= Nabalus altissimus (L.) Hook.

P. crepidinea Michx., Corymbed Rattlesnake-root, previous reports were based on a sight record by David Day, repudiated as to species by G. Clinton, collecting diary. A specimen was never collected by these men. Note the species is recorded for Erie and CattaraugusCo. by NYFA (July 2005) with indications that voucher specimens exist for these two counties.

$=$ Nabalus crepidineus (Michx.) DC.

Prenanthes serpentaria Pursh (excluded by Zander \& Pierce 1979)

P. trifoliata (Cass.) Fern., Gall-of-the-Earth

$=$ Nabalus trifoliolatus Cass.

$=\mathrm{N}$. trifoliatus of authors

$=\mathrm{N}$. trifoliatus var. dissectifolius Peck

PRIMULA L. (Primulaceae)

P. mistassinica Michx., Canadian Primrose $=\mathrm{P}$. mistassinica var. noveboracensis Fern.

Prinos verticillatus L. = Ilex verticillata (L.) Gray

PROBOSCIDEA Schmid. (Pedaliaceae)

*P. louisiana (Mill.) Woot. \& Standl., Unicornplant, Proboscis-flower, Ram's Horn $=$ Martynia proboscidea Glox.

= M. louisiana Mill.

$=$ Proboscidea louisianica, sensu Thell.

Prosartes lanuginosa (Michx.) Don $=$ Disporum lanuginosum ((Michx.) Nichols.

PROSERPINACA L. (Haloragaceae)

Proserpinaca intermedia Mackz. $=$ Proserpinaca palustris L.

P. palustris L., Mermaidweed var. crebra Fern. \& Grisc. var. palustris 
$=\mathrm{P}$. intermedia Mackz.

$=$ P. palustris var. latifolia Schindl.

$=$ P. platycarpa Small

Proserpinaca platycarpa Small $=$ Proserpinaca

palustris L. var. palustris

PRUNELLA L. (Lamiaceae)

P. vulgaris L., Heal-all

$=\mathrm{P}$. vulgaris var. lanceolata (Bart.) Fern .

PRUNUS L. (Rosaceae)

P. americana Marsh., Wild Plum, Hedge-plum, Sloe

Prunus autumnalis Liegel $=$ Prunus domestica L.

*P. avium (L.) L., Sweet Cherry, Bird-cherry, Mazzard

Prunus borealis (Michx.) Pursh = Prunus pensylvanica L.f.

*P. cerasus L., Sour Cherry, Pie-cherry

$=$ Cerasus vulgaris Mill.

*P. domestica L., Garden Plum

$=\mathrm{P}$. autumnalis Liegel

* var. domestica, Plum

*P. fruticosa Pallas, Ground-cherry, European Dwarf Cherry

Prunus lanceolata Willd. $=$ Prunus pensylvanica L.f.

*P. mahaleb L., Mahaleb-cherry, Mahaleb, Perfumed-cherry, St. Lucie-cherry

Prunus mollis sensu Torrey, not Dougl. = Prunus nigra Ait.

P. nigra Ait., Canada Plum, Wild Plum $=\mathrm{P}$. mollis sensu Torrey, not Dougl.

P. pensylvanica L.f., Pin-cherry, Fire-cherry, Wild Red Cherry, Bird-cherry

$=$ Cerasus pennsylvanica (L.f.) Loisel.

$=$ Prunus borealis (Michx.) Pursh

$=\mathrm{P}$. lanceolata Willd.

*P. persica (L.) Batsch, Peach

$=$ Amygdalus persica $\mathrm{L}$.

Prunus pumila var. cuneata (Raf.) Bailey $=$ Prunus pumila var. susquehanae (Hort. ex Willd.) Jaeger

P. pumila L., Sand-cherry

var. susquehanae (Hort. ex Willd.) Jaeger

$=$ P. pumila var. cuneata (Raf.) Bailey

$=\mathrm{P}$. susquehannae Hort. ex Willd.

P. serotina Ehrh., Black Cherry, Wild Cherry, Rum-cherry
$=\mathrm{P}$. virginiana L., in part

Prunus susquehanae Hort. ex Willd. = Prunus pumila var. susquehanae (Hort. ex Willd.) Jaeger

Prunus virginiana L., in part $=$ Prunus serotina Ehrh.

P. virginiana L., Choke Cherry

Psedera quinquefolia (L.) Greene $=$ P. quinquefolia (L.) Planch. ex DC.

Psedera quinquefolia var. hirsuta (Pursh) B. Robinson $=$ P. quinquefolia (L.) Planch. ex DC.

Pseudognaphalium macounii (Greene) Kartesz = Gnaphalium viscosum Kunth

PTELEA L. (Rutaceae)

P. trifoliata L., Hop-tree, Wafer-ash, Stinking Ash

Pteretis nodulosa $($ Michx.) Nieuwl. $=$ Matteuccia struthiopteris (L.) Todaro

Pteretis pensylvanica (Willd.) Fern. = Matteuccia struthiopteris (L.) Todaro

PTERIDIUM Gled. ex Scop. (Dennstaedtiaceae)

P. aquilinum (L.) Kuhn ex Decken, Eastern Bracken-fern, Bracken, Brake-fern, Brake var. latiusculum (Desv.) Underw. ex Heller $=\mathrm{P}$. aquilinum var. champlainense Boivin = P. latiusculum (Desv.) Hieron, not Maxon $=$ Pteris aquilina of American reports, not L. $=$ Pteris latiuscula Desv.

Pteris aquilina of American reports, not L. $=$ Pteridium aquilinum (L.) Kuhn ex Decken var. latiusculum (Desv.) Underw. ex Heller

Pteris latiuscula Desv. $=$ Pteridium aquilinum (L.) Kuhn ex Decken var. latiusculum (Desv.) Underw. ex Heller

PTEROSPORA Nutt. (Ericaceae)

P. andromedea Nutt., Pine-drops, Giant Bird'snest

$=$ Monotropa procera Torr. ex Eaton

$=\mathrm{P}$. andromeda of authors

PUCCINELLIA Parl. (Poaceae)

*P. distans (Jacq.) Parl., Alkali-grass

*ssp. borealis (Holmb.) W. E. Hughes = P. distans var. angustifolia (Blytt) Holmb.

*ssp. distans 
$=\mathrm{P}$. nuttalliana of NY reports, not (Schultes) Hitchc.

Puccinellia pallida (Torr.) Clausen in part $=$ Torreyochloa pallida (Torr.) Church var. pallida

PYCNANTHEMUM Michx. (Lamiaceae) Pycnanthemum flexuosum of some authors, not (Walt.) BSP. $=$ Pycnanthemum tenuifolium Schrad.

P. incanum (L.) Michx., Hoary Mountain-mint $=$ Koellia incana (L.) Kuntze

Pycnanthemum lanceolatum Pursh = Pycnanthemum virginianum (L.) Durand \& Jackson ex Fern. \& Robins.

Pycnanthemum linifolium (Willd.) Pursh = Pycnanthemum tenuifolium Schrad.

P. tenuifolium Schrad., Narrow-leaved Mountainmint

= Koellia flexuosa of NY reports, not (Walt.) MacM.

$=$ Pycnanthemum flexuosum of some authors, not (Walt.) Macm.

$=\mathrm{P}$. linifolium (Willd.) Pursh

P. torrei Benth., Torrey's Mountain-mint $=$ Koellia torrei (Benth.) Kuntze

P. verticillatum (Michx.) Pers., Mountain-mint var. verticillatum

P. virginianum (L.) Durieu \& Jackson ex Fern. \& B. Robinson, Virginia Mountain-mint

$=$ Koellia virginiana (L.) Kuntze

$=$ Pycnanthemum lanceolatum Pursh

PYROLA L. (Ericaceae)

P. americana Sweet, Round-leaved Pyrola $=\mathrm{P}$. rotundifolia Michx. ssp. americana (Sweet.) Clausen

$=\mathrm{P}$. rotundifolia Michx. var. americana

(Sweet.) Fern.

$=\mathrm{P}$. rotundifolia Michx. var. rotundifolia

P. asarifolia Michx., Bog-pyrola, Pink

Wintergreen

$=\mathrm{P}$. asarifolia var. incarnata (Fisch.) Fern. (of

Zenkert, 1934).

$=\mathrm{P}$. asarifolia var. purpurea (Bunge) Fern.

= P. uliginosa Torr. \& A. Gray

= P. rotundifolia var. uliginosa (Torr.) A. Gray
P. chlorantha Sw., Greenish-flowered Pyrola, Green Shinleaf, Wintergreen, = P. oxypetala Austin ex A. Gray "(with peloric flowers)", Mitchell \& Tucker, 1997.

$=$ P. virens Schweig. ex Schweig. \& Koerte

$=\mathrm{P}$. virens fo. paucifolia (Fern.) Fern.

$=\mathrm{P}$. chlorantha var. pauciflora (of Zenkert, 1934)

P. elliptica Nutt., Elliptic-leaved Pyrola, Shinleaf, Wild Lily-of-the-Valley

Pyrola oxypetala Austin $($ peloric $)=$ Pyrola chlorantha Sw.

Pyrola rotundifolia Michx. var. rotundifolia $=$ Pyrola americana Sweet

Pyrola rotundifolia Michx. var. americana (Sweet) Fern. $=$ Pyrola americana Sweet

Pyrola rotundifolia Michx. var. uliginosa (Torr.) A. Gray $=$ Pyrola asarifolia Michx.

Pyrola secunda L. = Orthilia secunda L.

Pyrola secunda L. var. pumila Paine $=$ Orthilia secunda L.

Pyrola secunda L. var. obtusata Turcz. $=$ Orthilia secunda L.

Pyrola uliginosa Torr. \& A. Gray = Pyrola asarifolia Michx.

Pyrola uniflora L. = Moneses uniflora (L.) A. Gray

Pyrola virens Schweig. ex Schweig. \& Koerte = Pyrola chlorantha Sw.

Pyrola virens fo. paucifolia (Fern.) Fern. = Pyrola chlorantha Sw.

PYRUS L. (Rosaceae)

Pyrus americana (Marsh.) DC. $=$ Sorbus americana Marsh.

Pyrus arbutifolia (L.) L.f. = Aronia arbutifolia (L.) Pers. (not Ell.)

Pyrus arbutifolia var. atropurpurea (Britt.) B.

Robinson $=[$ Aronia arbutifolia $\times$ melanocarpa $=$ A. $\times$ prunifolia (Marsh.)

Rehd.]

Pyrus arbutifolia var. erythrocarpa (Michx.) = Aronia arbutifolia (L.) Pers.

Pyrus arbutifolia var. melanocarpa Michx. = Aronia melanocarpa (Michx) Ell.

Pyrus aucuparia (L.) Gaertn. = Sorbus aucuparia L.

*P. communis L., Common Pear

Pyrus coronaria L. $=$ Malus coronaria (L.) Mill. 
Pyrus coronaria var. dasycalyx (Rehd.) Fern. =

Malus coronaria (L.) Mill.

Pyrus coronaria var. elongata (Rehd.) Bailey =

Malus coronaria (L.) Mill.

Pyrus coronaria var. lancifolia (Rehd.) Fern. =

Malus coronaria (L.) Mill.

Pyrus decora (Sarg.) Hyl. = Sorbus decora (Sarg.)

C. K. Schneid.

Pyrus dumosa Fern. = Sorbus decora (Sarg.) C. K. Schneid.

Pyrus floribunda Lindl. $=[$ Aronia arbutifolia $\times$ melanocarpa $=$ A. $\times$ prunifolia (Marsh.$)$

Rehd.]

Pyrus malus of authors, not L. = Malus pumila Mill.

QUERCUS L. (Fagaceae)

Q. alba L., White Oak

Q. alba $\times$ montana $=\mathbf{Q} \cdot \times$ saulii, Oak

Q. bicolor Willd., Swamp White Oak

Quercus borealis Michx. f. = Quercus rubra L.

Quercus borealis var. maxima (Marsh) Sarg. =

Quercus rubra L.

Q. coccinea Muenchh., Scarlet Oak

Recorded by NYFA but Jensen (1997)

does not record it for our region.

Quercus falcata of NY reports, not Michx. =

Quercus rubra L.

Q. ilicifolia Wang.; Scrub-oak, Bear-oak

Q. macrocarpa Michx., Bur Oak, Mossy-cup Oak, Northern Overcup Oak

Q. montana Willd., Chestnut-oak

= Q. prinus L. of most American authors, not L.

Q. muhlenbergii Engelm., Yellow Oak, Chinquapin Oak

= Q. prinoides var. acuminata Michx.

Quercus obtusiloba Michx. = Quercus stellata Wang.

Quercus pagoda of House's (1924) checklist, not Raf. = Quercus rubra L.

Q. palustris Muenchh., Pin Oak

Quercus prinoides var. acuminata Michx. not (Michx.) Gleason = Quercus muhlenbergii Engelm.

Quercus prinoides Willd. (excluded by Zander \& Pierce 1979)

Quercus prinus L., of most American authors = Quercus montana Willd.
Quercus prinus var. humilis Marsh. = Quercus prinoides Willd. (excluded by Zander \& Pierce 1979)

Q. rubra L., Red Oak

$=\mathrm{Q}$. borealis Michx. f.

$=$ Q. borealis var. maxima (Marsh.) Sarg.

$=$ Q. falcata of NY reports, not Michx.

$=$ Q. pagoda of House's (1924) checklist, not Raf.

= Q. rubra var. ambigua (A. Gray) Fern.

$=$ Q. rubra var. borealis (Michx. f.) Farw.

$=\mathrm{Q}$. triloba Michx.

Q. shumardii Buckl., Shumard Oak

Q. stellata Wang., Post Oak

$=$ Q. obtusiloba Michx.

Quercus tinctoria Bartr. = Quercus velutina Lam.

Quercus triloba Michx. = Quercus rubra L.

Q. velutina Lam., Black Oak, Dyer's Oak, Yellowbark Oak

$=\mathrm{Q}$. tinctoria Bartr.

Radicula hispida (Desv.) Britt. = Rorippa palustris (L.) Besser ssp. hispida (Desv.) Jonesell

Radicula sylvestris (L.) Druce $=$ Rorippa sylvestris (L.) Besser

Raimannia laciniata (Hill) Rose $=$ Oenothera laciniata Hill

RANUNCULUS L. (Ranunculaceae)

R. abortivus L., Small-flowered Crowfoot var. abortivus

var. eucyclus Fern.

Ranunculus acer L. = Ranunculus acris L.

*R. acris L., Tall Meadow-buttercup $=\mathrm{R}$. acer $\mathrm{L}$.

$=\mathrm{R}$. acris var. latisectus Beck

$=\mathrm{R}$. acris var. villosus (Drab.) Cole

Ranunculus alismaefolius (of Day, 1882) = Ranunculus ambigens S. Wats.

R. allegheniensis Britt., Alleghenian Crowfoot, Mountain-crowfoot

R. ambigens S. Wats., Water-plantain Spearwort, American Spearwort

$=\mathrm{R}$. alismaefolius (of Day, 1882)

$=\mathrm{R}$. laxicaulis (of authors)

$=\mathrm{R}$. obtusiusculus of authors, not Raf.

Ranunculus boraeanus Jord. $=$ Ranunculus recurvatus Poir. ex Lam.

Ranunculus brevicaulis Hook. $=$ Ranunculus rhomboideus Goldie 
*R. bulbosus L., Bulbous Buttercup, Meadowbloom

$=\mathrm{R}$. tuberosus Hornem.

Ranunculus circinatus of American authors, not Sibth. $=$ Ranunculus longirostris Godr.

Ranunculus cymbalaria Pursh $=($ excluded by Zander \& Pierce 1979)

Ranunculus delphinifolius Torr. $=$ Ranunculus flabellaris Raf. ex Bigel.

Ranunculus divaricatus Schrank = Ranunculus trichophyllus Chaix ex Vill.

R. fascicularis Muhl. ex Bigel., Early Buttercup

*R. ficaria L., Lesser Celandine $=$ Ficaria ficaria (L.) Karst.

$=\mathrm{R}$. ficaria ssp. bulbifera (Marsden-Jones) Lawal. ex Rob.

Ranunculus filiformis Michx. $=\mathrm{R}$. flammula L . var. filiformis (Michx.) Hooker

R. flabellaris Raf. ex Bigel., Yellow Watercrowfoot

$=\mathrm{R}$. delphinifolius Torr.

$=\mathrm{R}$. fluviatilis Bigel.

$=\mathrm{R}$. lacustris Beck \& Tracey

$=\mathrm{R}$. multifidus Pursh

$=\mathrm{R}$. purshii of NY reports, not Richards.

R. flammula L., Creeping Spearwort, Lesser Spearwort

var. filiformis (Michx.) Hooker

$=\mathrm{R}$. filiformis Michx.

$=\mathrm{R}$. reptans var. filiformis (L.) DC.

$=\mathrm{R}$. reptans var. reptans of authors, not $\mathrm{L}$.

Ranunculus fluviatilis Bigel. $=$ Ranunculus flabellaris Raf. ex Bigel.

Ranunculus hirsutus Muhl. = Ranunculus recurvatus Poir. ex Lam.

R. hispidus Michx., Hispid Buttercup

var. caricetorum (Greene) Duncan, Swamp- or Marsh-buttercup

$=\mathrm{R}$. septentrionalis of some authors, not Poir.

var. hispidus, Hispid Buttercup

var. nitidus (Muhl. ex Ell.) Duncan, Swamp-

$=\mathrm{R}$. septentrionalis Poir.

Ranunculus lacustris Beck \& Tracey = Ranunculus flabellaris Raf. ex Bigel.

Ranunculus laxicaulis (of authors) $=$ Ranunculus ambigens S. Wats.

R. longirostris Godr., Stiff White Water-crowfoot $=$ Batrachium longirostre (Godr.) F. Schultz
$=$ Ranunculus circinatus of American authors, not Sibth.

$=\mathrm{R}$. longirostris of authors

$=\mathrm{R}$. subrigidus Drew

Ranunculus multifidus Pursh $=$ Ranunculus flabellaris Raf. ex Bigel.

Ranunculus obtusiusculus of authors, not Raf. = Ranunculus ambigens S. Wats.

R. pensylvanicus L. f., Bristly Crowfoot

Ranunculus purshii of NY reports, not Richards. = Ranunculus flabellaris Raf. ex Bigel.

Ranunculus purshii Richards. = not sensu R. flabellaris Raf. ex Bigel, but Ranunculus gmelinii DC. (excluded by Zander \& Pierce 1979)

R. recurvatus Poir. ex Lam., Hooked Crowfoot, Rough Buttercup

$=\mathrm{R}$. boraeanus Jord.

$=\mathrm{R}$. hirsutus Muhl.

$=\mathrm{R}$. recurvatus var. adpressipilus Weath.

*R. repens L., Creeping Buttercup

$=\mathrm{R}$. repens var. degeneratus Schur

$=\mathrm{R}$. repens var. pleniflorus Fern.

Ranunculus reptans var. filiformis (L.) DC. = Ranunculus flammula L. var. filiformis (Michx.) Hooker

Ranunculus reptans var. reptans of authors, not L. $=\mathrm{R}$. flammula L. var. filiformis (Michx.) Hooker

Ranunculus rhomboideus Goldie, Prairie Buttercup, Regl. M. Niag. Oldham 1999 $=\mathrm{R}$. brevicaulis Hook.

*R. sceleratus L., Cursed Crowfoot, Blisterwort

Ranunculus septentrionalis of some authors, not Poir. ex Lam. = R. hispidus Michx. var. caricetorum (Greene) Duncan, or var. nitidus (Muhl. ex Ell.) Duncan

Ranunculus subrigidus Drew $=$ Ranunculus longirostris Godr.

[R. trichophyllus Chaix ex Vill., White Watercrowfoot (possibly excluded)]

$=$ Batrachium trichophyllum (Chaix ex Vill.) Bosch.

$=\mathrm{R}$. aquatilis var. calvescens (Drew) Benson

$=\mathrm{R}$. aquatilis var. trichophyllus (Chaix) Gray

$=\mathrm{R}$. aquatilis var. capillaceus (Thuill.) DC. of Zenkert (1934)

$=\mathrm{R}$. divaricatus Schrank 
According to records at NYS, there are no records of specimens in any of the western New York counties of this species (NYFA 1990).

Ranunculus tuberosus Hornem. $=$ Ranunculus bulbosus L.

RAPHANUS L. (Brassicaceae)

*R. raphanistrum L., Jointed Charlock, Wild Radish, White Charlock

*R. sativus L., Garden Radish

RATIBIDA Raf. (Asteraceae)

*R. pinnata (Vent) Barnh., Gray-headed Coneflower, Prairie-coneflower $=$ Lepachys pinnata (Vent.) Torr. \& A. Gray

Razoumofskya pusilla (C. Peck) Kuntze $=$ Arceuthobium pusillum C. Peck

Reynoutria japonica Houtt. $=$ Polygonum cuspidatum Sieb. \& Zucc.

Reynoutria sachalinensis (Schmidt ex Maxim.) Nakai ex Mori $=$ Polygonum sachalinense Schmidt ex Maxim.

RESEDA L. (Resedaceae)

*R. lutea L., Yellow Mignonette, Scrambling Rocket

RHAMNUS L. (Rhamnaceae)

R. alnifolia L'Hér. , Alder-leaved Buckthorn

Rhamnus caroliniana of old NY reports, not Walt. $=$ Rhamnus frangula $\mathrm{L}$.

*R. cathartica L., Common Buckthorn

*R. frangula L., Alder Buckthorn, Smooth Buckthorn

$=$ Frangula alnus Mill.

= Rhamnus caroliniana of old NY reports, not Walt.

RHODODENDRON L. (Ericaceae)

R. groenlandicum (Oeder) Kron \& Judd, Laborador Tea

= Ledum decumbens of early NY reports, not (Ait.) Lodd.

$=\mathrm{L}$. groenlandicum Oeder

$=$ L. latifolium Ait.

$=$ L. palustre ssp. groenlandicum (Oeder) Hultén
R. maximum L., Rhododendron, Great Laurel, Rosebay, White Laurel

Rhododendron nudiflorum (L.) Torrey of Zenkert $\&$ Zander $(1975)=$ Rhododendron periclymenoides (Michx.) Shinners

Rhododendron nudiflorum var. roseum (Loisel.) Weig. $=$ Rhododendron prinophyllum (Small) Millais

R. periclymenoides (Michx.) Shinners, Pinksterflower, Pinkster-bloom, Purple Honeysuckle, Election-pink $=$ Azalea nudiflora L.

$=\mathrm{A}$. periclymenoides Michx.

$=$ R. nudiflorum (L.) Torrey of Zenkert \& Zander (1975)

R. prinophyllum (Small) Millais, Pink Azalea, Early Azalea, Mountain-azalea, Rosehoneysuckle, Election-pink

$=$ Azalea prinophylla Small

= Rhododendron nudiflorum var. roseum (Loisel.) Weig.

$=\mathrm{R}$. roseum (Loisel.) Rehd.

Rhododendron roseum (Loisel.) Rehd. = Rhododendron prinophyllum (Small) Millais

R. viscosum (L.) Torrey, Swamp Azalea

RHODOTYPOS Sieb. \& Zucc. (Rosaceae)

Rhodotypos kerrioides Sieb. \& Zucc. =

Rhodotypos scandens (Thunb.) Makino

*R. scandens (Thunb.) Makino, Jetbead, White Kerria

$=$ R. kerrioides Sieb. \& Zucc.

$=$ R. tetrapetala (Sieb.) Makino

Rhodotypos tetrapetala (Sieb.) Makino = Rhodotypos scandens (Thunb.) Makino

RHUS L. (Anacardiceae)

R. aromatica Ait., Fragrant Sumac, Lemon

Sumac, Polecat-bush

$=\mathrm{R}$. canadensis Marsh.

$=\mathrm{R}$. trilobata of NY reports, not (Nutt.) A. Gray

R. copallinum L., Shining Sumac, Winged Sumac, Dwarf Sumac

Rhus canadensis Marsh = Rhus aromatica Ait.

Rhus cotinus L. $=$ Cotinus coggygria Scop.

R. glabra L., Smooth Sumac 
R. hirta (L.) Sudworth, Staghorn-sumac, Velvetsumac

$=$ Datisca hirta L.

$=$ Rhus typhina L.

Rhus radicans L. $=$ Toxicodendron radicans (L.)

Kuntze ssp. radicans

Rhus radicans var. rydbergii (Small ex Rydb.)

Rehd. $=$ Toxicodendron rydbergii (Small ex Rydb.) Greene

Rhus radicans var. vulgaris (Michx.) DC. in part $=$

Toxicodendron rydbergii (Small ex Rydb.)

Greene

Rhus toxicodendron (of Zenkert, 1934) $=$

Toxicodendron radicans (L.) Kuntze s.l.

Rhus trilobata of NY reports, not (Nutt.) A. Gray =

Rhus aromatica Ait.

Rhus typhina L. = Rhus hirta (L.) Sudworth

Rhus venenata DC. $=$ Toxicodendron vernix $(\mathrm{L}$.

Kuntze

Rhus vernix L. $=$ Toxicodendron vernix $(\mathrm{L}$.

Kuntze

RHYNCHOSPORA Vahl (Cyperaceae)

R. alba (L.) Vahl, White Beaked-rush

R. capillacea Torr., Capillary Beaked-rush

$=$ R. capillacea var. laeviseta E. J. Hill

RIBES L. (Grossulariaceae)

R. americanum Mill., Wild Black Currant

$=\mathrm{R}$. floridanum L'Hér.

*R. aureum Pursh, Golden Currant, Buffalocurrant

*var. villosum DC.

$=$ Ribes odoratum Wendl.f. ex Bartl. \& Wendl. f.

R. cynosbati L., Prickly Gooseberry, Dogberry, Pasture-gooseberry

$=$ Grossularia cynosbati (L.) Mill.

Ribes floridanum L'Hér. = Ribes americanum Mill.

R. glandulosum Grauer, Skunk-currant

$=\mathrm{R}$. prostratum L'Hér.

R. hirtellum Michx., Smooth Gooseberry

$=\mathrm{R}$. hirtellum var. saxosum (Hooker) Fern.

$=\mathrm{R}$. oxyacanthoides L., in part

R. lacustre (Pers.) Poir., Swamp Black Currant, Bristly Black Currant

$=\mathrm{R}$. oxyacanthoides L. var. lacustre Pers.

*R. nigrum L., Garden Black Currant
Ribes odoratum Wendl. f. ex Bartl. \& Wendl. f. = Ribes aureum Pursh var. villosum DC.

Ribes oxyacanthoides (of authors) $=$ Ribes hirtellum Michx.

Ribes oxyacanthoides L. var. lacustre Pers. = Ribes lacustre (Pers.) Poir.

Ribes prostratum L'Hér. = Ribes glandulosum Grauer

*R. rotundifolium Michx., Eastern Wild Gooseberry, Round-leaf Currant

*R. rubrum L., Red Currant, Northern or Garden Red Currant, Cherry-currant

$=\mathrm{R}$. sativum (Reichenb.) Syme

$=\mathrm{R}$. vulgare of NY reports, not Lam.

Ribes rubrum var. subglandulosum Maxim. = Ribes triste Pallas

Ribes sativum (Reichenb.) Syme $=$ Ribes rubrum L.

R. triste Pallas, Wild Red Currant $=\mathrm{R}$. triste var. albinervium (Michx.) Fern. $=\mathrm{R}$. rubrum var. subglandulosum Maxim.

Ribes vulgare of NY reports, not Lam. $=$ Ribes rubrum $\mathrm{L}$.

ROBINIA L. (Leguminosae)

Robinia fertilis of NY reports, not Ashe $=$ Robinia hispida $\mathrm{L}$.

*R. hispida L., Bristly Locust, Rose-acacia, Mossy Locust

$=\mathrm{R}$. fertilis of NY reports, not Ashe

$=\mathrm{R}$. hispida var. fertilis of NY reports, not (Ashe) R. Clausen

*R. pseudo-acacia L., Black Locust, False Acacia

*R. viscosa Vent. ex Vauq., Clammy Locust

RORIPPA Scop. (Brassicaceae)

Rorippa aquatica (Eaton) E. J. Palmer \& Steyermark $=$ Armoracia lacustris $($ A. Gray) Al-Shehbaz \& V. Bates

Rorippa islandica (Oeder) Borbás ssp. islandica (Oeder) Borbás $=$ Rorippa palustris ssp. palustris (L.) Besser

Rorippa islandica (Oeder) Borbás var. fernaldiana Butters \& Abbe = Rorippa palustris (L.) Besser ssp. fernaldiana (Butters \& Abbe) Jonesell

Rorippa islandica (Oeder) Borbás var. hispida (Desv.) Butters \& Abbe = Rorippa 
palustris (L.) Besser ssp. hispida (Desv.) Jonesell

*R. nasturtium-aquaticum (L.) Hayek, Watercress

= Nasturtium officinale R. Br. ex Ait.

$=\mathrm{N}$. nasturtium-aquaticum (L.) Karst.

$=$ Sisymbrium nasturtium-aquaticum L.

Rorippa palustris (L.) Besser = Rorippa palustris (L.) Besser ssp. palustris

R. palustris (L.) Besser, Marsh-cress, Yellow Water-cress

ssp. fernaldiana (Butters \& Abbe) Jonesell

$=\mathrm{R}$. islandica (Oeder) Borbás var. fernaldiana Butters \& Abbe

ssp. hispida (Desv.) Jonesell, Hispid Yellow Cress

$=$ Radicula hispida (Desv.) Britt.

$=\mathrm{R}$. islandica var. hispida (Desv.) Butters \& Abbe

*ssp. palustris

$=\mathrm{R}$. islandica ssp. islandica (Oeder) Borbás

$=\mathrm{R}$. palustris $(\mathrm{L}$.) Besser

*R. sylvestris (L.) Besser, Yellow Cress, Creeping Yellow Ccress

$=$ Radicula sylvestris (L.) Druce

ROSA L. (Rosaceae)

R. acicularis Lindl., Prickly Rose

ssp. sayi (Schwein. ex Keating) Lewis

$=$ Rosa acicularis var. bourgeauiana Crépin

$=\mathrm{R}$. acicularis var. sayiana Erl.

$=\mathrm{R}$. sayi Schwein. ex Keating

R. acicularis $\times$ blanda $=\mathbf{R} . \times$ housei Erlanson, House's Rose

Rosa acicularoides Schuette $=$ Rosa blanda Ait.

Rosa bicknellii Rydb. = Rosa virginiana Mill.

R. blanda Ait., Smooth Rose, Meadow-rose

$=\mathrm{R}$. acicularoides Schuette

$=\mathrm{R}$. blanda varieties of NY reports

$=\mathrm{R}$. blanda var. glabra Crépin

$=\mathrm{R}$. fraxinifolia Lindl.

$=\mathrm{R}$. solanderi Tratt.

*R. canina L., Dog-rose

R. carolina L., Dwarf Rose var. carolina

$=\mathrm{R}$. carolina var. glandulosa (Crépin) Farw.

$=\mathrm{R}$. carolina var. grandiflora (Baker) Rehd.

$=\mathrm{R}$. carolina var. obovata (Raf.) Deam

$=\mathrm{R}$. gemella Willd.
$=\mathrm{R}$. housei Erl.

$=\mathrm{R}$. obovata Raf.

$=\mathrm{R}$. parviflora Ehrh.

$=\mathrm{R}$. serrulata Raf.

Rosa carolinensis Marsh. $=$ Rosa virginiana Mill.

*R. cinnamomea L., Damask Rose

$=\mathrm{R}$. spinosissima L., in part, a nomen ambiguum (Mitchell \& Tucker 1997)

*R. eglanteria L., Sweetbrier, Eglantine $=\mathrm{R}$. rubiginosa $\mathrm{L}$.

*R. gallica L., French Rose

Rosa gemella Willd. = Rosa carolina L. var. carolina

Rosa housei Erl. = Rosa carolina L. var. carolina

Rosa lucida Ehrh. = Rosa virginiana Mill.

*R. micrantha Borr. ex Sm. in Sowerby, SmallFlowered Sweetbrier

$=\mathrm{R}$. rubiginosa var. micrantha Lindl.

*R. multiflora Thunb. ex Murr., Multiflora Rose

Rosa nanella Rydb. = Rosa virginiana Mill.

Rosa obovata Raf. $=$ Rosa carolina L. var. carolina

R. palustris Marsh., Swamp Rose, Églantier

$=$ R. palustris var. inermis (Schuette) Erl.

Rosa parviflora Ehrh. = Rosa carolina L.

Rosa rubifolia R. Br. ex Ait. = Rosa setigera

Michx. var. tomentosa Torr. \& A. Gray

Rosa rubiginosa L. $=$ Rosa eglanteria L .

Rosa rubiginosa var. micrantha Lindl. = Rosa

micrantha Borr. ex Sm. in Sowerby

*R. rugosa Thunb., Rugose Rose, Japanese or

Turkistan Rose, Sand-Rose, Sea-beach

Rose

Rosa sayi Schwein. ex Keating = Rosa acicularis

Lindl. ssp. sayi (Schwein. ex Keating)

Lewis

Rosa serrulata Raf. = Rosa carolina L. var. carolina

R. setigera Michx., Prairie-rose, Climbing Rose var. setigera

*var. tomentosa Torr. \& A. Gray

$=\mathrm{R}$. rubifolia R. Br. ex Ait.

Rosa solanderi Tratt. $=$ Rosa blanda Ait.

Rosa spinosissima L., in part, a nomen ambiguum

(Mitchell \& Tucker 1997) $=$ Rosa

cinnamomea L.

R. virginiana Mill., Pasture-rose, Wild Rose

$=\mathrm{R}$. bicknellii Rydb.

$=\mathrm{R}$. carolinensis Marsh.

$=\mathrm{R}$. lucida Ehrh.

$=\mathrm{R}$. nanella Rydb. 
RUBUS Tourn. (Rosaceae)

R. allegheniensis Porter ex Bailey, Common Highbush Blackberry, Northern Blackberry, Sow-teat Blackberry $=\mathrm{R}$. villosus (of local authors) $=$ Rubus allegheniensis Porter ex Bailey (p.p.)

Rubus americanus Britt. $=$ Rubus pubescens Raf.

* R. bifrons Vest ex Tratt., Himalaya-berry $=\mathrm{R}$. discolor of some authors, not Weihe \& Nees

$=\mathrm{R}$. procerus of some authors, not Müll.

R. canadensis L. Smooth Blackberry $=$ R. pergratus Blanch. of Eaton \& Schrot (1987).

Rubus dalibarda L. $=$ Dalibarda repens L.

(*)R. flagellaris Willd. Northern Dewberry, American Dewberry, Prickly Dewberry (considered native by NYFA July 2005)

$=\mathrm{R}$. canadensis (of local authors)

$=\mathrm{R}$. villosus var. humifusus (of authors)

Rubus heterophyllus (of American authors) $=$ Rubus pensilvanicus Poir. ex Lam.

R. hispidus L. Running Blackberry, Swampdewberry

$=\mathrm{R}$. permixtus Blanch. of Eaton and Schrot (1987).

R. idaeus L. European Red Raspberry

\section{*ssp. idaeus}

ssp. strigosus (Michx.) Focke, Wild Raspberry

$=\mathrm{R}$. idaeus ssp. sachalinensis (Levl.) Focke

$=\mathrm{R}$. idaeus ssp. melanolasius Focke

$=\mathrm{R}$. idaeus var. strigosus (Michx.) Maxim.

$=\mathrm{R}$. strigosus Michx.

$=\mathrm{R}$. strigosus var. canadensis (Richards.) House

Rubus idaeus americanus Torrey $=$ Rubus occidentalis L.

*R. illecebrosus Focke, Balloonberry, Raspberrystrawberry

*R. laciniatus Willd. Cut-leaved Blackberry, Evergreen Blackberry

R. occidentalis L., Black Raspberry, Black-cap, Black Thimbleberry

$=\mathrm{R}$. idaeus americanus Torrey

$=\mathrm{R}$. occidentalis var. flavobaccus Blanch.

R. odoratus L. Purple-flowering Raspberry, Pink Thimbleberry
R. pensilvanicus Poir. ex Lam., Highbush

Blackberry

$=\mathrm{R}$. heterophyllus Willd.

Rubus pergratus Blanch. $=$ Rubus canadensis L .

Rubus permixtus Blanch. $=$ Rubus hispidus L.

sensu lato

*R. phoenicolasius Maxim., Wineberry

R. pubescens Raf., Dwarf Raspberry

$=\mathrm{R}$. americanus Britt.

$=$ R. pubescens var. pilosifolius A. Hill

$=\mathrm{R}$. triflorus Richards.

$=\mathrm{R}$. triphyllus Thunb.

R. setosus Bigel., Northern Bog-blackberry

Rubus strigosus Michx. = Rubus idaeus L. ssp. strigosus (Michx.) Focke

Rubus strigosus var. canadensis (Richards.) House $=$ Rubus idaeus L. ssp. strigosus (Michx.) Focke

Rubus triflorus Richards. = Rubus pubescens Raf. Rubus triphyllus Thunb. = Rubus pubescens Raf.

Rubus villosus (of local authors) $=$ Rubus allegheniensis Porter ex Bailey (p.p.)

Rubus villosus var. humifusus (of authors) $=$ Rubus flagellaris Willd.

RUDBECKIA L. (Asteraceae)

R. hirta L.

*var. pulcherrima Farw., Black-eyed Susan, Gloriosa Daisy, Yellow Daisy

$=$ R. serotina Nutt.

(*)R. laciniata L., Tall Coneflower, Black-eyedSusan, Golden-Glow (considered native by NYFA July 2005)

$=\mathrm{R}$. laciniata var. bipinnata Perdue

$=\mathrm{R}$. laciniata var. hortensis Bailey

Rudbeckia laciniata var. hortensis Bailey $=$ Rudbeckia laciniata L.

Rudbeckia serotina Nutt. = Rudbeckia hirta L. var. pulcherrima Farw.

(*)R. triloba L., Thin-leaved Coneflower, Blackeyed Susan, Yellow Daisy (the var. triloba is considered to be not native by NYFA July 2005)

RUMEX L. (Polygonaceae)

Rumex $\times$ pratensis Mert. $\&$ Koch $=[$ Rumex crispus $\times$ obtusifolius $=\times$ acutus L.]

*R. acetosella L., Sheep Sorrel 
$=\mathrm{R}$. acetosella ssp. angiocarpus (Murb.) Murb.

$=\mathrm{R}$. acetosella L. ssp. pyrenaicus (Pourret ex Lapeyr.) Akeroyd

$=\mathrm{R}$. angiocarpus Murb.

R. altissimus Wood, Tall, Pale, Water- or Peachleaf Dock

Rumex angiocarpus Murb. $=$ Rumex acetosella L.

Rumex britannica (of authors) $=$ Rumex orbiculatus A. Gray

Rumex conglomeratus of NY reports, not Murr. = Rumex obtusifolius L.

Rumex crispatulus Michx. = Rumex obtusifolius L.

*R. crispus L., Curled Dock, Yellow or Sour Dock

$* \mathbf{R}$. crispus $\times$ obtusifolius $=\mathbf{R}$. acutus L., Curly Dock

$=\mathrm{R} . \times$ pratensis Mert. \& Koch

Rumex fueginus Phil. = Rumex maritimus L. var. fueginus (Phil.) Dusén

Rumex hydrolapathum var. americanum A. Gray = Rumex orbiculatus A. Gray

R. maritimus L., Golden Dock, Seashore-dock var. fueginus (Phil.) Dusén

$=\mathrm{R}$. fueginus Phil.

Rumex mexicanus Meisn. in part $=$ Rumex salicifolius Weinm. var. mexicanus (Meisn.) Hitchc.

*R. obtusifolius L., Broad-leaved Dock, Bitterdock, Red-veined Dock

$=\mathrm{R}$. conglomeratus of NY reports, not Murr.

$=\mathrm{R}$. crispatulus Michx.

$=\mathrm{R}$. pulcher of NY reports, not L.

$=$ R.sanguineus of NY reports, not L.

R. orbiculatus A. Gray, Great Water-dock

$=\mathrm{R}$. britannica of authors, not L.

$=\mathrm{R}$. hydrolapathum var. americanum A. Gray

$=\mathrm{R}$. orbiculatus var. borealis Rech. f.

*R. patientia L., Garden-patience, Patience-dock, Monk's Rhubarb

Rumex pulcher of NY reports, not L. = Rumex obtusifolius L.

*R. salicifolius Weinm., Willow-leaved Dock var. mexicanus (Meisn.) Hitchc.

$=\mathrm{R}$. mexicanus Meisn. ex DC.

$=\mathrm{R}$. salicifolius Weinm. ssp. triangulivalvis Danser

$=\mathrm{R}$. salicifolius Weinm. var. triangulivalvis (Danser) Hickm.
$=\mathrm{R}$. triangulivalvis (Danser) Rech. $\mathrm{f}$.

Rumex sanguineus of NY reports, not L. $=$ Rumex obtusifolius L.

Rumex triangulivalvis (Danser) Rech. f. $=$ Rumex salicifolius Weinm. var. mexicanus (Meisn.) Hitchc.

R. verticillatus L., Water- or Swamp-dock

RUPPIA L.(Ruppiaceae)

R. maritima L., Ditch-grass

$=$ R. maritima var. longipes Hagstr.

$=$ R. maritima var. onondagensis Fern. \& Wieg.

$=\mathrm{R}$. maritima var. rostrata Agardh

Rynchospora (ortho. var.) = Rhynchospora Vahl

SAGINA L. (Caryophyllaceae)

*S. procumbens L., Pearlwort, Bird's Eye

SAGITTARIA L. (Alismataceae)

S. cuneata Sheldon, Arum-leaved Arrowhead, Wapeto

Sagittaria engelmaniana ssp. longirostra (Micheli) Bogin = Sagittaria latifolia Willd .

Sagittaria esculenta Howell $=$ Sagittaria latifolia Willd.

S. graminea Michx., Grass-leaf Sagittaria $=\mathrm{S}$. eatonii $\mathrm{Sm}$.

Sagittaria heterophylla Pursh $=$ Sagittaria rigida Pursh

Sagittaria heterophylla var. rigida (Pursh) Engelm. in A. Gray $=$ Sagittaria rigida Pursh

S. latifolia Willd., Broad-leaved Arrowhead

$=\mathrm{S}$. engelmaniana ssp. longirostra (Micheli) Bogin

$=\mathrm{S}$. esculenta Howell

$=$ S. latifolia var. obtusa (Muhl. ex Willd.) Sm.

$=\mathrm{S}$. variabilis Engelm. in A. Gray

S. rigida Pursh, Sessile-fruited Arrowhead

$=\mathrm{S}$. heterophylla Pursh

$=\mathrm{S}$. heterophylla var. rigida (Pursh) Engelm. in A. Gray

Sagittaria variabilis Engelm. in A. Gray = Sagittaria latifolia Willd.

SALICORNIA L. (Chenopodiaceae)

S. europaea L., Glasswort, Samphire, Chickenclaws 
$=$ S. europaea var. simplex (Pursh) Fern.

$=\mathrm{S}$. herbacea $\mathrm{L}$.

$=$ S. prostrata Pall.

Salicornia europaea var. simplex (Pursh) Fern. =

Salicornia europaea L.

Salicornia herbacea L. = Salicornia europaea L.

Saliornia prostrata Pall. $=$ Salicornia europaea L .

SALIX L. (Salicaceae)

*S. alba L., White Willow

= S. alba var. vitellina (L.) Koch

$=\mathrm{S}$. vitellina $\mathrm{L}$.

S. amygdaloides Anderss., Peach-leaved Willow

Salix angustissima Anderss. $=$ Salix exigua Nutt.

*S. babylonica L., Weeping Willow $=\mathrm{S}$. babylonica varieties

S. babylonica $\times$ fragilis $=S . \times$ pendulina

Wenderoth, Wisconsin Weeping Willow

$=\mathrm{S}$. babylonica $\times$ fragilis

$=\mathrm{S}$. blanda Anderss.

$=\mathrm{S} . \times$ blanda

S. bebbiana Sarg., Beaked Willow, Bebb's Willow = S. depressa L. var. rostrata (Anderss.) Hiit. $=$ S. livida var. occidentalis (of Day, 1882)

$=\mathrm{S}$. rostrata Richards., not Thuill.

Salix blanda Anderss. $=[$ Salix babylonica $\times$ fragilis $=\mathrm{S} . \times$ pendulina Wenderoth $]$

S. candida Fluegge ex Willd., Hoary Willow, Sage Willow

$=$ S. candida var. denudata Anderss.

$=\mathrm{S}$. pellita of NY reports, not (Anderss.) Schneid.

Salix coactilis Fern. = Salix sericea Marsh.

S. cordata Michx., Sand-dune Willow, Heartleafwillow

$=\mathrm{S}$. syrticola Fern.

Salix cordata Muhl., not Michx., of Zenkert, 1934

$=$ Salix eriocephala Michx.

Salix cordata angustata Anders, sec. House $1924=$ Salix eriocephala Michx.

Salix depressa L. var. rostrata (Anderss.) Hiit. = Salix bebbiana Sarg.

S. discolor Muhl., Pussy-willow, Glaucous Willow

S. eriocephala Michx., Heartleaf-willow, Stiff Willow, Diamond Willow

= S. cordata Muhl., not Michx., of Zenkert, 1934

$=\mathrm{S}$. cordata angustata Anders., sec. House 1924
$=\mathrm{S}$. discolor $\times$ rigida of $\mathrm{NY}$ reports

$=\mathrm{S}$. nigra $\times$ petiolaris of NY reports

$=\mathrm{S}$. nigra $\times$ rigida of NY reports

$=\mathrm{S}$. rigida Muhl.

$=\mathrm{S}$. myricoides of NY reports, not Muhl.

S. exigua Nutt., Sandbar-willow

$=\mathrm{S}$. angustissima Anderss.

$=\mathrm{S}$. interior Rowlee

$=\mathrm{S}$. linearifolia Rydb.

$=\mathrm{S}$. longifolia Muhl.

*S. fragilis L., Crack Willow, Brittle Willow Salix glaucophylloides Fern. $=$ Salix myricoides Muhl.

Salix gracilis Anderss. $=$ Salix petiolaris Sm. Salix grisea Willd. = Salix sericea Marsh .

S. humilis Marsh., Prairie-willow, Gray Willow, Small Pussy-willow, Upland Willow

var. humilis

var. tristis (Ait.) Griggs

$=\mathrm{S}$. tristis Ait.

Salix interior Rowlee $=$ Salix exigua Nutt.

Salix linearifolia Rydb. = Salix exigua Nutt.

Salix livida var. occidentalis (of Day, 1882) = Salix bebbiana Sarg.

Salix longifolia Muhl. $=$ Salix exigua Nutt.

S. lucida Muhl., Shining or Glossy Willow

Salix myrtilloides (of authors) $=$ Salix pedicellaris Pursh

*S. matsudana Koidz., Dragon-claw Willow, Corkscrew-willow

S. myricoides Muhl.

$=$ Salix glaucophylloides Fern.

A specimen noted from the Fort Erie area of the Regional Municipality of Niagara by

Soper and Heimberger (1982). Its range extends north to the James Bay area from dunes, gravelly shores and flats around the Great Lakes' shores. Mitchell and Tucker (1997) indicate that New York reports of S. myricoides are S. eriocephala Michx. The species does not appear to occur in New York State.

S. nigra Marsh., Black Willow, Swamp-willow

S. pedicellaris Pursh, Bog-willow

$=\mathrm{S}$. myrtilloides (of authors)

$=\mathrm{S}$. myrtilloides ssp. pedicellaris (Pursh) Anderss.

$=\mathrm{S}$. pedicellaris var. hypoglauca Fern. 
Salix pellita of NY reports, not (Anderss.) Schneid. $=$ Salix candida Fluegge ex Willd.

*S. pentandra L., Bay-leaf Willow

S. petiolaris Sm., Slender Willow

$=\mathrm{S}$. gracilis Anderss.

Salix petiolaris var. grisea (Willd.) Torr. $=$ Salix sericea Marsh.

*S. purpurea L., Basket-willow, Purple Willow Salix rigida Muhl. $=$ Salix eriocephala Michx.

Salix rostrata Richards., not Thuill. = Salix bebbiana Sarg.

S. sericea Marsh., Silky Willow

$=\mathrm{S}$. coactilis Fern.

$=\mathrm{S}$. grisea Willd.

$=\mathrm{S}$. petiolaris var. grisea (Willd.) Torr.

S. serissima (Bailey) Fern., Autumn-willow

Salix syrticola Fern. = S. cordata Michx.

Salix tristis Ait. $=$ Salix humilis Marsh.

Salix vitellina $\mathrm{L} .=$ Salix alba $\mathrm{L}$.

SALSOLA L. (Chenopodiaceae)

Salsola atriplicifolium Spreng. $=$ Cycloloma atriplicifolium (Spreng.) Coult.

*S. pestifer A. Nelson, Russian Thistle

$=\mathrm{S}$. australis of authors, not R. Br.

$=\mathrm{S}$. iberica Sennen \& Pau

$=$ S. kali L. var. tenuifolia Tausch, not Meyer

$=$ S. ruthenica Iljin ex Sorn.

$=\mathrm{S}$. tragus of authors, not $\mathrm{L}$.

Salvia officinalis L. (excluded by Zander and

Pierce 1979).

SAMBUCUS L. (Caprifoliaceae)

S. canadensis L., Black Elderberry

= S. nigra L. ssp. canadensis (L.) R. Bolli

Sambucus pubens Michx. = Sambucus racemosa L . ssp. pubens (Michx.) House

Sambucus nigra L. ssp. canadensis (L.) R. Bolli = Sambucus Canadensis L.

S. racemosa L., Red-berried Elder

ssp. pubens (Michx.) House

$=\mathrm{S}$. pubens Michx.

$=\mathrm{S}$. racemosa var. leucocarpa (Torr. \& A. Gray) Cronq.

SAMOLUS L. (Primulaceae)

Samolus floribundus Kunth $=$ Samolus valerandii

L. ssp. parviflorus (Raf.) Hultén
Samolus parviflorus Raf. $=$ Samolus valerandii L . ssp. parviflorus (Raf.) Hultén

S. valerandii L., Brookweed

ssp. parviflorus (Raf.) Hultén

$=$ S. floribundus Kunth

$=$ S. parviflorus Raf.

$=\mathrm{S}$. valerandii var. americanus A. Gray

Samolus valerandii var. americanus A. Gray $=$ Samolus valerandii L. ssp. parviflorus (Raf.) Hultén

SANGUINARIA L. (Papaveraceae)

S. canadensis L., Bloodroot, Puccoon

SANGUISORBA L. (Rosaceae)

S. canadensis L., Burnet

*S. minor Scop., Garden Burnet

$=$ Poterium sanguisorba of authors, not L.

= S. muricata (Spach) Gremeli

$=\mathrm{S}$. minor ssp. muricata Briq.

Sanguisorba muricata $($ Spach $)$ Gremeli $=$ Sanguisorba minor Scop.

SANICULA L. (Apiaceae)

S. canadensis L., Short-styled Snakeroot $=\mathrm{S}$. canadensis var. grandis Fern.

Sanicula gregaria Bickn. $=$ Sanicula odorata (Raf.) Pryer \& Phillippe

S. marilandica L., Black Snakeroot

S. odorata (Raf.) Pryer \& Phillippe, Clustered Snakeroot

$=\mathrm{S}$. gregaria Bickn.

= Triclinium odoratum Raf.

S. trifoliata Bickn., Large-fruited Snakeroot

SAPONARIA L. (Caryophyllaceae)

Saponaria hispanica Mill. $=$ Vaccaria hispanica (Mill.) Raush.

*S. officinalis L., Bouncing Bet, Soapwort, Fuller's-herb, Sheepweed, Hedge-pink

Saponaria vaccaria L. $=$ Vaccaria hispanica (Mill. $)$ Raush.

Sarothamnus scoparius (L.) Wimmer = Cytisus scoparius (L.) Link

SARRACENIA L. (Sarraceniaceae)

S. purpurea L., Pitcher-plant, Side-saddle Plant

SASSAFRAS Nees (Lauraceae) 
S. albidum (Nutt.) Nees, Sassafras

$=$ S. officinale Nees \& Eberm.

Sassafras officinale Nees \& Eberm. = Sassafras albidum (Nutt.) Nees

SATUREJA L. (Lamiaceae)

Satureja acinos (L.) Scheele $=$ Acinos arvensis (Lam.) Dandy

Satureja arkansana (Nutt.) Briq. = Calamintha arkansana (Nutt.) Shinners

Satureja glabella var. angustifolia (Torr.) Svenson = Calamintha arkansana (Nutt.) Shinners

Satureja glabra (Nutt.) Fern. = Calamintha arkansana (Nutt.) Shinners

*S. hortensis L., Summer-savory

Satureja vulgaris (L.) Fritsch = Clinopodium vulgare L.

SAURURUS L. (Saururaceae)

S. cernuus L., Lizard-tail

SAXIFRAGA L. (Saxifragaceae)

S. aizoides L., Yellow Mountain-saxifrage

S. pensylvanica L., Swamp-saxifrage

S. virginiensis Michx., Early Saxifrage

SCABIOSA L. (Dipsacaceae)

Scabiosa arvensis L. = Knautia arvensis (L.) Coulter

Scabiosa australis Wulf. $=$ Succisella inflexa (Kluk) Beck

*S. columbaria L., Pigeon-scabious, Pincushionflower, Scabious

Schedonorus pratensis (Huds.) P. Beauv. = Lolium pratense (Hudson) S. Darbyshire

SCHEUCHZERIA L. (Juncaginaceae)

S. palustris L., Bog Arrow-grass ssp. americana (Fern.) Hultén $=\mathrm{S}$. palustris var. americana Fern.

SCHIZACHNE Hack. (Poaceae)

S. purpurascens (Torr.) Swallen, Purple Oat, False Melic = Melica purpurascens (Torr.) Hitchc.

SCHIZACHYRIUM Nees (Poaceae)

S. scoparium (Michx.) Nash, Little Bluestem ssp. scoparium
= Andropogon scoparius Michx.

= A. scoparius var. septentrionalis Fern. \& Grisc.

$=$ A. scoparius var. frequens Hubbard

= A. scoparius var. glaucescens House

Schoenoplectus acutus (Muhl.) Á. \& D. Löve = Scirpus acutus Muhl. ex Bigel.

Schoenoplectus pungens (Vahl) Dur. \& Jacks = Scirpus pungens Vahl

Schoenoplectus tabernaemontanii (Gmel.) Palla $=$ Scirpus tabernaemontanii Gmel.

Schoenoplectus torreyi (Olney) Palla $=$ Scirpus torreyi Olney

Schollera graminea (Michx.) Raf. $=$ Heteranthera dubia (Jacq.) MacM.

SCILLA L. (Liliaceae)

*S. siberica Haw. ex Andr., Scilla, Squill

SCIRPUS L. (Cyperaceae)

S. acutus Muhl. ex Bigel., Western Bulrush, Hardstemmed Bulrush, Tule

= Schoenoplectus acutus (Muhl.) Á. \& D. Löve

S. americanus Pers., Chair-maker's Rush, Threesquare, Swordgrass, $=\mathrm{S}$. olneyi A. Gray

Scirpus americanus Pers. in part $=$ Scirpus pungens Vahl.

Scirpus atrocinctus Fern. $=$ Scirpus cyperinus $($ L. $)$ Kunth

S. atrovirens Willd. (not Muhl.), Dark-green Bulrush

$=\mathrm{S}$. atrovirens var. sycnocephalus Cowles

S. cespitosus L., Tufted Club-rush

$=$ Trichophorum caespitosum (L.) Hartm.

Scirpus campestris var. paludosus (A. Nels.) Fern. $=$ Scirpus maritimus L.

S. clintonii A. Gray, Clinton's Club-rush

$=$ Trichophorum clintonii (A. Gray) S. G. Sm.

S. cyperinus (L.) Kunth, Wool-grass

$=\mathrm{S}$. atrocinctus Fern.

$=\mathrm{S}$. cyperinus var. pelius Fern.

$=\mathrm{S}$. eriophorum Michx.

$=$ S. pedicellatus Fern.

$=\mathrm{S}$. rubricosus Fern.

Scirpus eriophorum Michx. $=$ Scirpus cyperinus (L.) Kunth 
S. expansus Fern., Wood-bulrush

$=\mathrm{S}$. sylvaticus L. in part

S. fluviatilis (Torr.) A. Gray, River-bulrush

$=$ Bolboschoenus fluviatilis (Torrey) Soják

S. georgianus Harper, Bulrush

$=\mathrm{S}$. atrovirens var. georgianus (Harper) Fern. in part.

S. heterochaetus Chase, Slender Bulrush

S. hudsonianus (Michx.) Fern., Alpine Bulrush, Alpine Cottongrass

$=$ Eriophorum alpinum L.

$=$ Trichophorum alpinum (L.) Pers.

Scirpus lacustris ssp. tabernaemontanii (Gmel.) Syme $=$ Scirpus tabernaemontanii Gmel.

Scirpus lineatus of authors, not Michx. = Scirpus pendulus Muhl.

S. maritimus L., Seaside-bulrush, Bayonet-grass

$=$ Bolboschoenus maritimus (L.) Palla ssp. paludosus (A. Nelson) T. Koyama

$=$ Scirpus campestris var. paludosus $(\mathrm{A}$. Nels.) Fern.

$=\mathrm{S}$. maritimus var. fernaldii (Bickn.) Beetle

$=$ S. maritimus var. paludosus (A. Nels.)

Kuekenth.

$=\mathrm{S}$. paludosus A. Nels.

$=\mathrm{S}$. paludosus var. atlanticus Fern.

S. microcarpus C. Presl, Red-tinged Bulrush

$=$ Scirpus rubrotinctus Fern.

Scirpus olneyi A. Gray = Scirpus americanus Pers.

Scirpus paludosus A. Nels. = Scirpus maritimus L.

Scirpus pauciflorus Lightf. $=$ Eleocharis pauciflora (Lightf.) Link

Scirpus pedicellatus Fern. $=$ Scirpus cyperinus $($ L.) Kunth

S. pendulus Muhl., Reddish Bulrush

$=\mathrm{S}$. lineatus of authors, not Michx.

Scirpus planifolius Muhl. $=$ Scirpus verecundus Fern.

S. polyphyllus Vahl, Leafy Bulrush

S. pungens Vahl, Bulrush

$=$ Schoenoplectus pungens (Vahl) Dur. \& Jacks

Scirpus rubricosus Fern. $=$ Scirpus cyperinus (L.) Kunth

Scirpus rubrotinctus Fern. $=$ Scirpus microcarpus C. Presl

S. smithii Gray, Bulrush

$=\mathrm{S}$. juncoides Roxb. var. williamsii (Fern.)

T. Koyama
$=\mathrm{S}$. purshianus Fern.

$=$ S. smithii var. williamsii (Fern.) Beetle

Scirpus sylvaticus L., in part $=$ Scirpus expansus Fern.

S. tabernaemontanii Gmel., Great Bulrush, Softstem Bulrush, Tule, Great or Southern Bulrush

$=$ Schoenoplectus tabernaemontanii $(\mathrm{Gmel}$. Palla

$=$ Scirpus lacustris ssp. tabernaemontanii

(Gmel.) Syme

$=\mathrm{S}$. validus Vahl

S. torreyi Olney, Torrey's Bulrush

$=$ Schoenoplectus torreyi (Olney) Palla

Scirpus validus Vahl = Scirpus tabernaemontanii Gmel.

S. verecundus Fern., Wood Club-rush $=$ Trichophorum planifolium (Spreng.) Palla $=$ Scirpus planifolius Muhl.

SCLERANTHUS L. (Caryophyllaceae)

*S. annuus L., Knawel, German Knot-Grass $=\mathrm{S}$. annuus ssp. ruscinonensis (Gillot \& Coste) P. Sell

$=\mathrm{S}$. uncinatus Schur

SCLERIA Berg. (Cyperaceae)

S. reticularis Michx., Nutrush, Whipgrass var. reticularis

$=\mathrm{S}$. muhlengergii Steud.

$=\mathrm{S}$. setacea Poir.

Scleria muhlengergii Steud. $=$ Scleria reticularis Michx. var. reticularis

Scleria setacea Poir. $=$ Scleria reticularis Michx. var. reticularis

S. verticillata Muhl. ex Willd., Low Nut-rush

SCROPHULARIA L. (Scrophulariaceae)

S. lanceolata Pursh, Hare-figwort

S. marilandica L., Maryland Figwort, Carpenter'ssquare

*Scrophularia nodosa L. (excluded by Zander \& Pierce 1979)

SCUTELLARIA L. (Lamiaceae)

Scutellaria australis (Fassett) Epling = Scutellaria parvula Michx. var. australis Fassett 
Scutellaria australis var. leonardii of NY reports, not $($ Epling $)$ Fern $=$ Scutellaria parvula Michx. var. parvula

Scutellaria canescens Nutt. $=$ Scutellaria incana Biehl.

Scutellaria epilobiifolia Hamil. = Scutellaria galericulata L.

S. galericulata L., Hooded Scullcap $=$ S. epilobiifolia Hamilt.

S. incana Biehl., Hoary Skullcap $=\mathrm{S}$. canescens Nutt.

S. lateriflora L., Mad-dog Scullcap

S. parvula Michx., Small Scullcap var. australis Fassett $=\mathrm{S}$. australis (Fassett) Epling var. parvula

$=\mathrm{S}$. australis var. leonardii of NY reports, not (Epling) Fern.

$=\mathrm{S}$. parvula var. missouriensis (Torr.) Goodman \& Lawson

SECALE L. (Poaceae)

*S. cereale L., Rye

SEDUM L. (Crassulaceae)

*S. acre L., Mossy Stoncrop, Yellow Sedum, Wall-sedum, Wallpepper

Sedum purpureum (L.) Link = Sedum telephium L.

*S. sarmentosum Bunge, Sedum

*S. telephium L., Live-forever

=Hylotelephium telephium (L.) H. Ohba.

$=$ Sedum purpureum (L.) Link

$=$ S. triphyllum (Haw.) S.F. Gray

*S. ternatum Michx., Wild Stonecrop, Stonewort, Mountain-stonecrop

Sedum triphyllum (Haw.) S.F. Gray $=$ Sedum telephium L.

SELAGINELLA Beauv. (Selaginellaceae)

S. apoda L., Creeping Selaginella, Northern Meadow-spikemoss

$=$ Lycopodium apodum L.

= Selaginella apus (L.) Spring ex Mart.

$=\mathrm{S}$. eclipes Buck

Selaginella apus (L.) Spring ex Mart. = Selaginella apoda (L.) Spring

Selaginella eclipes Buck = Selaginella apoda L.

S. rupestris (L.) Spring, Rock-selaginella, Rockspikemoss
= Lycopodium rupestre L.

Selinum canadense Michx. $=$ Conioselinum chinense (L.) BSP.

SENECIO L. (Asteraceae)

S. aureus L., Golden Ragwort $=$ Packera aurea (L.) A. \& D. Löve

$=$ Senecio aureus var. gracilis (Pursh) Britt.

S. aureus $\times$ pauperculus, Groundsel

Senecio aureus var. balsamitae (Muhl.) Torr. \& A. Gray $=$ Senecio pauperculus Michx.

Senecio aureus var. gracilis (Pursh) Britt. = Senecio aureus L.

Senecio balsamitae Muhl. $=$ Senecio pauperculus Michx.

S. obovatus Muhl. ex Willd., Round-leaved Squaw-weed, Ragwort, Groundsel, Squawweed

= Packera obovata (Muhl. ex Willd.) W. A. Weber \& A. Löve

S. pauperculus Michx., Balsam-groundsel

$=$ Packera paupercula (Michx.) Á. \& D. Löve

$=$ Senecio pauperculus varieties

$=$ S. pauperculus var. balsamitae (Muhl.) Fern.

$=\mathrm{S}$. balsamitae Muhl.

*S. viscosus L., Sticky Groundsel

*S. vulgaris L., Common Groundsel

SENNA Mill. (Fabaceae)

S. hebecarpa (Fern.) Irwin \& Barneby, Wild Senna, Partridge-pea, American senna $=$ Cassia hebecarpa Fern.

$=\mathrm{C}$. marylandica of NY reports, not L.

Serapias helleborine (L.) Crantz = Epipactis helleborine (L.) Crantz

Seriococarpus asteroides (L.) BSP $=$ Aster paternus Cronq.

SETARIA Beauv. (Poaceae)

*S. faberi Herrm., Giant Foxtail

Setaria glauca (L.) Beauv. = Setaria pumila (Poir.) Schultes

*S. italica (L.) Beauv., Italian Millet

Setaria lutescens (Weigel) Hubbard = Setaria pumila (Poir.) Schultes

*S. pumila (Poir.) Schultes, Pigeon-grass, Yellow Foxtail

$=$ Pennisetum glaucum (L.) R. Br. 
$=$ S. glauca $($ L.) Beauv.

$=\mathrm{S}$. lutescens (Weigel) Hubbard

*S. verticillata (L.) Beauv., Bristly Foxtail

*S. viridis (L.) Beauv., Green Foxtail, Bottlegrass (probably originally Bottle-brush Grass).

SHEPHERDIA Nutt. (Elaeagnaceae)

S. canadensis (L.) Nutt., Canadian Buffalo-berry, Soapberry

SHERARDIA L. (Rubiaceae)

*S. arvensis L., Field-madder

SICYOS L. (Cucurbitaceae)

S. angulatus L., Bur-cucumber, Star-cucumber Sicyos lobata Michx. = Echinocystis lobata

(Michx.) Torr. \& A. Gray

SIDA L. (Malvaceae)

Sida abutilon L. = Abutilon theophrasti Medik.

*S. spinosa L., Prickly Mallow

SILENE L. (Caryophyllaceae)

Silene alba (Mill.) Krause = Silene latifolia Poir.

S. antirrhina L., Sleepy Catchfly

$=\mathrm{S}$. antirrhina var. depauperata Rydb.

$=\mathrm{S}$. antirrhina var. divaricata B. Robinson

$=$ S. divaricata (B. Robinson) Smyth

*S. armeria L., Sweet William Catchfly, Not-soPretty

$=\mathrm{S}$. armeria var. sparsiflora Schur

*S. cserei Baumg., Campion, Balkan Catchfly

$=\mathrm{S}$. czerei and S. cserii of authors

= S. fabaria sensu Rydb., not (L.) Sibth. \&

Sm.

Silene cucubalus Wibel $=$ Silene vulgaris

(Moench) Garcke

Silene czerei of authors $=$ Silene cserei Baumg.

*S. dichotoma Ehrh., Forking Catchfly, Forked Catch-fly

$=\mathrm{S}$. gallica of NY reports, not L.

Silene divaricata (B. Robinson) Smyth $=$ Silene antirrhina L.

Silene fabaria sensu Rydb., not (L.) Sibth. \& Sm. = Silene cserei Baumg.

Silene gallica of NY reports, not L. = Silene dichotoma Ehrh.

Silene inflata (Salisb.) Sm. = Silene vulgaris (Moench) Garcke
*S. latifolia Poir., Evening-lychnis

$=$ Lychnis alba Mill.

= L. arvensis Gaertn., Meyer \& Scherb.

$=\mathrm{L}$. vespertina Sibth.

$=$ Silene alba (Mill.) Krause

$=$ S. pratensis (Raf.) Gord. \& Gren.

Silene latifolia (Mill.) Britt. \& Rendle, not Poir. (of Zenkert, 1934) $=$ Silene vulgaris $($ Moench $)$ Garcke

*S. noctiflora L., Night-flowering Catchfly, Sticky Campion

$=$ Melandrium noctiflorum (L.) Fries

Silene pratensis (Raf.) Gord. \& Gren. = Silene latifolia Poir.

S. stellata (L.) Ait. f., Starry Campion, Widow's Frill

$=$ S. stellata var. scabrella (Nieuwl.) Palmer \& Steyerm.

*S. vulgaris (Mornch) Garcke, Bladder Campion, Maiden's Tears

$=\mathrm{S}$. cucubalus Wibel

= S. latifolia (Mill.) Britt. \& Rendle, not Poir. (of Zenkert, 1934)

$=$ S. inflata (Salisb.) Sm.

SILPHIUM L. (Asteraceae)

*S. lacinatum L., Compass-plant, Rosinweed

*S. perfoliatum L., Cup-plant

*S. trifoliatum L., Whorled Rosinweed

$=\mathrm{S}$. asteriscus in part

SIL YBUM Adans. (Asteraceae)

S. marianum (L.) Gaertn., Milk-thistle, Blessed

Thistle, St. Mary's Thistle

$=$ Carduus mariana L.

= Mariana mariana (L.) Hill

SINAPIS L. (Brassicaceae)

*S. arvensis L., Charlock

$=$ Brassica arvensis (L.) Rabenh.

$=\mathrm{B}$. arvensis var. schkuhriana (Reichenb.)

Wheeler

= B. kaber (DC.) L. Wheeler

= B. kaber var. pinnatifida (Stokes) Wheeler

$=$ Sinapis schkuhriana Reichenb.

Sinapis nigra L. = Brassica nigra (L.) Koch

Sinapis schkuhriana Reichenb. $=$ Sinapis arvensis

L. 
SISYMBRIUM L. (Brassicaceae)

*S. altissimum L., Tumble Mustard

= Norta altissima (L.) Britt. ex Britt. \& Brown

Sisymbrium brachycarpon Rich. ex Frankl. = Descurainia pinnata (Walt.) Britt. ssp. brachycarpa (Richards. ex Frankl.) Detl.

Sisymbrium brachycarpum Richardson $=$ Descurainia pinnata (Walt.) Britt. ssp. brachycarpa (Richards. ex Frankl.) Detl.

Sisymbrium canescens Nutt. of Ontario reports and Western New York = Descurainia pinnata var. pinnata (Walt.) Britt. (excluded by Zander and Pierce 1979).

Sisymbrium erucastrum Poll. $=$ Erucastrum gallicum (Willd.) Schulz

Sisymbrium gallicum Willd. $=$ Erucastrum gallicum (Willd.) Schulz

*S. loeselii L., Tall Hedge-mustard

Sisymbrium murale L. $=$ Diplotaxis muralis $(\mathrm{L}$. DC.

Sisymbrium nasturtium-aquaticum L. = Rorippa nasturtium-aquaticum (L.) Hayek

*S. officinale (L.) Scop., Hedge-mustard, Bankcress

$=$ Erysimum officinale L.

$=$ Sisymbrium officinale var. leiocarpum DC.

Sisymbrium sophia L. = Descurainia sophia (L.) Webb ex Prantl

Sisymbrium tenuifolia (L.) DC. $=$ Diplotaxis tenuifolia (L.) DC.

Sisymbrium thalianum (L.) J.Gay = Arabidopsis thaliana (L.) Heynh.

SISYRINCHIUM L. (Irideaceae)

S. angustifolium Mill., Narrow-leaved Blue-eyed Grass

$=\mathrm{S}$. graminoides Bickn.

Sisyrinchium gramineum Curtis $=$ Sisyrinchium angustifolium Mill.

Sisyrinchium graminoides Bickn. $=$ Sisyrinchium angustifolium Mill.

S. montanum Greene, Mountain Blue-eyed Grass var. montanum

S. mucronatum Michx., Michaux's Blue-eyed Grass

SIUM L. (Apiaceae)
Sium carsonii Durieu ex A. Gray = Sium suave Walt.

Sium cicutaefolium Schrank $=$ Sium suave Walt.

S. suave Walt., Water-parsnip

$=$ S. carsonii Durieu ex A. Gray

$=\mathrm{S}$. cicutaefolium Schrank

$=\mathrm{S}$. lineare Michx.

Sium lineare Michx. = Sium suave Walt.

Smallanthus uvedalius (L.) Mackz. ex Small = Polymnia uvedalia (L.) L.

Smilacina bifolia Torr. $=$ Maianthemum canadensis Desf.

Smilacina racemosa (L.) Desf. $=$ Maianthemum racemosum $\mathrm{L}$.

Smilacina racemosa var. cylindrata Fern. $=$ Maianthemum racemosum L.

Smilacina stellata (L.) Desf. = Maianthemum stellatum $\mathrm{L}$.

Smilacina trifolia (L.) Desf. = Maianthemum trifolium L.

SMILAX L. (Liliaceae)

S. herbacea L., Carrion-flower, Jacob's Ladder $=\mathrm{S}$. lasioneuron of NY reports, not Hook.

S. hispida Muhl. ex Torr., Bristly Greenbrier, Hagbrier

$=$ S. tamnoides var. hispida (Muhl.) Fern.

Smilax lasioneuron of NY reports, not Hook. = Smilax herbacea L.

S. rotundifolia L., Catbrier, Greenbrier, Horsebrier, Bull-brier

Smilax tamnoides var. hispida (Muhl.) Fern. = Smilax hispida Muhl. ex Torr.

SOLANUM L. (Solanaceae)

Solanum americanum Mill. in part $=$ Solanum nigrum $\mathrm{L}$.

Solanum americanum Mill. in part $=$ Solanum ptychanthum Dunal

*S. carolinense L., Horse-nettle, Ball Night-shade

Solanum cornutum of authors, not Lam. = Solanum rostratum Dunal

*S. dulcamara L., Bitter Nightshade, Climbing or Trailing Nightshade, Bittersweet

*S. nigrum L., Black Nightshade, Poison-berry $=\mathrm{S}$. americanum Mill. in part

$=\mathrm{S}$. rubrum of authors, not $\mathrm{L}$.

Solanum nigrum of NY reports in large part, not L. $=$ Solanum ptychanthum Dunal 
Solanum lycopersicum L. = Lycopersicon esculentum Mill.

S. ptychanthum Dunal, Black Nightshade, Poisonberry

$=\mathrm{S}$. americanum of some NY reports, not Mill.

$=\mathrm{S}$. nigrum of NY reports in large part, not L.

*S. rostratum Dunal, Buffalo-bur, Kansas Thistle

$=\mathrm{S}$. cornutum of authors, not Lam.

Solanum rubrum of authors, not L. $=$ Solanum nigrum $\mathrm{L}$.

Solea concolor Forst. $=$ Hybanthus concolor (Forst.) Spreng.

SOLIDAGO L. (Asteraceae)

Solidago altissima L. = Solidago canadensis var. scabra (Muhl.) Torr. \& A. Gray

Solidago altissima var. procera (Ait.) Fern. = Solidago canadensis var. scabra (Muh.) Torr. \& A. Gray

S. arguta Ait., Cut-leaved Goldenrod $=$ S. muhlenbergii Torr. \& A. Gray

Solidago aspera Ait. $=$ Solidago rugosa Mill. ssp. aspera (Ait.) Cronq.

S. bicolor L., Silverrod, White Goldenrod

Solidago bicolor var. concolor Torr. \& A. Gray = Solidago hispida Muhl. ex Willd. (excluded by Zander \& Pierce 1979)

S. caesia L., Blue-stemmed Goldenrod

S. canadensis L., Canada or Common Goldenrod var. canadensis

$=\mathrm{S}$. rupestris of NY reports, not Raf. var. hargeri Fern., Harger's Goldenrod var. scabra (Muhl.) Torr. \& A. Gray, Tall Goldenrod

$=\mathrm{S}$. altissima $\mathrm{L}$.

$=$ S. altissima var. procera (Ait.) Fern.

$=\mathrm{S}$. Canadensis var. procera Torrey \& A. Gray

S. flexicaulis L., Zigzag Goldenrod $=\mathrm{S}$. latifolia L.

S. gigantea Ait., Late Goldenrod $=\mathrm{S}$. gigantea var. leiophylla Fern.

$=\mathrm{S}$. gigantea var. serotina (Ait.) Cronq.

$=\mathrm{S}$. serotina Ait.

$=$ S. serotina var. gigantea (Ait.) A. Gray

Solidago graminifolia (L.) Salisb. = Euthamia graminifolia (L.) Nutt. ex Cass.
Solidago graminifolia var. nuttallii (Greene) Fern. $=$ Euthamia graminifolia (L.) Nutt. ex Cass.

Solidago graminifolia var. polycephala (Fern.) Fern. $=$ Euthamia graminifolia (L.) Nutt. ex Cass.

S. hispida Muhl. ex Willd., Goldenrod

S. houghtonii Torr. \& A. Gray ex A. Gray, Houghton's Goldenrod

$=\mathrm{S}$. ohioensis $\times$ ptarmicoides of some authors

Solidago humilis (of authors $)=$ Solidago uliginosa Nutt.

S. juncea Ait., Early Goldenrod

Solidago lanceolata L. = Euthamia graminifolia (L.) Nutt. ex Cass.

Solidago lateriflora L. = Aster lateriflorus (L.) Britt. var. lateriflorus

Solidago latifolia L. = Solidago flexicaulis L .

Solidago muhlenbergii Torr.\& A. Gray $=$ Solidago arguta Ait.

Solidago neglecta Torr.\& A. Gray = Solidago uliginosa Nutt.

S. nemoralis Ait., Gray Goldenrod, Old-field Goldenrod

S. odora Ait., Sweet Goldenrod $=$ S. suaveolens of authors, not Schoepf.

S. ohioensis Riddell, Ohio Goldenrod

Solidago ohioensis $\times$ ptarmicoides of some authors $=$ Solidago houghtonii Torr. \& A. Gray ex A. Gray

S. patula Muhl. ex Willd., Rough-leaved Goldenrod, Spreading Goldenrod

Solidago polycephala Fern. $=$ Euthamia graminifolia (L.) Nutt. ex Cass.

S. ptarmicoides (Nees) Boivin, Upland-goldenrod, Upland White Aster

= Aster ptarmicoides (Nees) Torr. \& A. Gray

Solidago purshii Porter $=$ Solidago uliginosa Nutt. var. linoides (Torrey \& A. Gray) Fern.

S. rigida L., Stiff Goldenrod

S. rugosa Mill., Rough Goldenrod ssp. aspera (Ait.) Cronq., Rough Goldenrod $=\mathrm{S}$. rugosa var. aspera (Ait.) Fern.

$=\mathrm{S}$. aspera Ait.

ssp. rugosa

var. rugosa

Solidago rupestris of NY reports, not Raf. $=$ Solidago canadensis L. var. canadensis 
S. sempervirens L., Seaside-goldenrod

Solidago serotina Ait. $=$ Solidago gigantea Ait. Solidago serotina var. gigantea (Ait.) A. Gray = Solidago gigantea Ait.

S. squarrosa Muhl., Stout Ragged Goldenrod [Solidago stricta Ait. (a far southern species excluded by Zander \& Pierce, 1979)]

Solidago suaveolens of authors, not Schoepf. = Solidago odora Ait.

S. uliginosa Nutt., Bog- or Swamp-goldenrod var. linoides (Torrey \& A. Gray) Fern. var. uliginosa

$=\mathrm{S}$. humilis (of authors)

$=$ S. neglecta Torr. \& A. Gray

$=$ S. uniligulata (DC.) Porter

$=$ S. uniligulata var. neglecta (Torr.\& A. Gray) Fern.

$=\mathrm{S}$. uniligulata var. levipes Fern.

S. ulmifolia Muhl. ex Willd., Elm-leaved Goldenrod

Solidago uniligulata (DC.) Porter $=$ Solidago uliginosa Nutt.

Solidago uniligulata var. levipes Fern. = Solidago uliginosa Nutt.

Solidago uniligulata var. neglecta (Torr.\& A. Gray) Fern. $=$ Solidago uliginosa Nutt.

SONCHUS L. (Asteraceae)

S. arvensis L., Sow-thistle, Milk-thistle, Hogthistle

*ssp. arvensis Field Sow-thistle

*ssp. uliginosus (Bieb.) Nyman, Marsh Sowthistle

= S. arvensis var. glabrescens Guenth., Graeb. \& Wimm.

$=\mathrm{S}$. uliginosus Bieb.

*S. asper (L.) Hill, Spiny-leaved Sow-thistle

*S. oleraceus L., Common Sow-thistle, Milkthistle

Sonchus uliginosus Bieb. $=$ Sonchus arvensis L. ssp. uliginosus (Bieb.) Nyman

Sophora tinctoria L. = Baptisia tinctoria (L.) Vent.

SORBARIA (Sér.) A. Br. (Rosaceae)

*S. sorbifolia (L.) A. Br., False Spiraea $=$ Spiraea sorbifolia L.

SORBUS L. (Rosaceae)
S. americana Marsh., American Mountain-ash, Dogberry, Roundwood $=$ Pyrus americana (Marsh.) DC.

$=$ Sorbus microcarpa Pursh

*S. aucuparia L., Rowan Tree, Mountain-ash, Quickbeam

$=$ Pyrus aucuparia (L.) Gaertn.

S. decora (Sarg.) C.K. Schneid., Showy Mountainash, Dogberry

$=$ Pyrus decora (Sarg.) Hyland

$=$ P. dumosa Fern.

= Sorbus sambucifolia of NY reports, not

(Cham. \& Schlect.) Roemer

Sorbus microcarpa Pursh $=$ Sorbus americana Marsh.

Sorbus sambucifolia of NY reports, not (Cham. \& Schlect.) Roemer $=$ Sorbus decora (Sarg.) Schneid.

SORGHASTRUM Nash (Poaceae)

S. nutans (L.) Nash ex Small, Indian-grass $=$ Sorgum nutans $($ L.) A. Gray

SORGHUM Moench (Poaceae)

*S. bicolor (L.) Moench, Sorghum, Broomcorn $=$ Holcus sorghum L.

*S. halepense (L.) Pers., Johnson-grass $=$ Holcus halepensis L.

Sorghum nutans (L.) A. Gray = Sorghastrum nutans (L.) Nash

SPARGANIUM L. (Sparganianceae)

S. americanum Nutt., Nuttall's Bur-reed $=\mathrm{S}$. simplex var. nuttalii Engelm.

S. androcladum (Engelm.) Morong, Bur-reed

Sparganium chlorocarpum Rydb. $=$ Sparganium erectum L.

Sparganium emersum Rehm. $=$ Sparganium erectum L.

S. erectum L., Green-fruited Bur-reed

$=\mathrm{S}$. chlorocarpum Rydb.

$=$ S. emersum Rehm.

$=$ S. simplex Hudson

S. eurycarpum Engelm. ex A. Gray, Giant Burreed, Great Bur-reed

$=\mathrm{S}$. ramosum Pursh

Sparganium ramosum Pursh $=$ Sparganium eurycarpum Engelm. ex A. Gray 
Sparganium simplex Hudson $=$ Sparganium erectum L.

Sparganium simplex var. nuttalii Engelm. = Sparganium americanum Nutt.

SPARTINA Schreb. (Poaceae)

Spartina cynosuroides (of authors) $=$ Spartina pectinata Link

Spartina michauxiana Hitchc. $=$ Spartina pectinata Link

*S. patens (Ait.) Muhl., Salt-meadow Grass, Highwater Grass

$=\mathrm{S}$. patens var. juncea Hitchc.

$=\mathrm{S}$. patens var. monogyna (Curtis) Fern.

S. pectinata Link, Slough-grass, Freshwater or Prairie-cordgrass

$=\mathrm{S}$. cynosuroides (of authors)

$=\mathrm{S}$. michauxiana Hitchc.

$=$ S. pectinata var. suttiei (Farw.) Fern.

Spartium scoparium L. = Cytisus scoparius (L.) Link

Spathyema foetida (L.) Raf. = Symplocarpus foetidus (L.) Salisb. ex Nutt.

Specularia perfoliata (L.) A. DC. $=$ Triodanis perfoliata (L.) Nieuwl.

SPERGULA L. (Caryophyllaceae)

S. arvensis L., Corn-spurry

$=\mathrm{S}$. arvensis var. sativa (Bönn.) Mert. \&

Koch (not Reichenb.)

$=\mathrm{S}$. linicola Boreau

$=\mathrm{S}$. maxima Weihe

$=\mathrm{S}$. sativa Bönn.

$=\mathrm{S}$. vulgaris Bönn.

Spergula linicola Boreau $=$ Spergula arvensis L . Spergula maxima Weihe $=$ Spergula arvensis L . Spergula sativa Bönn. $=$ Spergula arvensis L . Spergula vulgaris Bönn. $=$ Spergula arvensis L .

SPERGULARIA (Pers.) J. \& C. Presl (Caryophyllaceae)

Spergularia alata Wieg. $=$ Spergularia media (L.) C. Presl ex Griseb.

Spergularia alata Wieg. in part $=$ Spergularia salina J. \& C. Presl

Spergularia campestris (L.) Aschers. = Spergularia rubra (L.) J. \& C. Presl

*S. media (L.) C. Presl ex Griseb., Margined Sand-spurry
= Alsine marginata (DC. ex Lam. \& DC.) Reichenb.

= A. media (L.) Druce

= Arenaria marginata DC. ex Lam. \& DC.

= A. maritima Pall.

$=$ A. media L.

$=$ Spergularia alata Wieg.

$=$ S. marginata (DC. ex Lam. \& DC.) Kittel

$=$ S. maritima (All.) Choiv.

Spergularia canina $($ Leffler $)$ Leffler $=$ Spergularia salina J. \& C. Presl

Spergularia leiosperma (Kindb.) F. Schmidt $=$ Spergularia salina J. \& C. Presl

Spergularia marginata (DC. ex Lam. \& DC.) Kittel = Spergularia media (L.) C. Presl ex Griseb.

Spergularia marina (L.) Griseb. $=$ Spergularia salina J. \& C. Presl

Spergularia maritima (All.) Choiv. $=$ Spergularia media (L.) C. Presl ex Griseb.

*S. rubra (L.) J. \& C. Presl, Common Sandspurry, Purple Sand-spurry, Sandwort

$=$ Alsine rubra (L.) Crantz

= Arenaria rubra L.

$=$ Spergularia campestris (L.) Aschers.

*S. salina J. \& C. Presl, Saltmarsh Sand-spurry

$=$ Alsine maritima Pall.

= Arenaria rubra var. marina $\mathrm{L}$.

$=$ A. marina Allioni

= Buda marina (L.) Dumort.

$=$ Spergula salina $($ J. \& C. Presl) Dietr.

$=$ Spergularia alata Wieg. in part

$=\mathrm{S}$. canina (Leffler) Leffler

= S. leiosperma (Kindb.) F. Schmidt

$=$ S. marina (L.) Griseb.

$=\mathrm{S}$. tenue Greene

$=$ Tissa marina (L.) Britt.

Spergularia tenue Greene $=$ Spergularia salina J. \& C. Presl

SPHENOPHOLIS Scribn. (Poaceae)

Sphenopholis intermedia (Rydb.) Rydb. = Sphenopholis obtusata (Michx.) Scribn. var. major (Torr.) Erdm.

S. nitida (Biehler) Scribn., Early Bunchgrass, Wedgegrass

S. obtusata (Michx.) Scribn., Prairie Wedgegrass $=$ Eatonia obtusata A. Gray 
var. major (Torr.) Erdm., Intermediate Bunchgrass

$=$ Eatonia pennsylvanica A. Gray, in part.

$=$ E. pennsylvanica A. Gray var. major

$=$ S. intermedia (Rydb.) Rydb.

$=\mathrm{S} . \times$ pallens of NY reports, not (Biehl.) Scribn.

Sphenopholis obtusata var. lobata (Trin.) Scribn. = Sphenopholis obtusata (Michx.) Scribn. var. obtusata

Sphenopholis obtusata var. pubescens (Scribn. \& Merr.) Scribn. $=$ Sphenopholis obtusata (Michx.) Scribn. var. obtusata

Sphenopholis $\times$ pallens of NY reports, not (Biehl.) Scribn. $=$ Sphenopholis obtusata (Michx.) Scribn. var. major (Torr.) Erdm.

S. pensylvanica (L.) Hitchc., Swamp-oats

$=$ Trisetum pensylvanicum (L.) Beauv. ex R. $\& \mathrm{~S}$.

Spinulum annotinum (L.) A. Haines = Lycopodium annotinum L. var. pungens (La Pyl.) Desv.

Spinulum canadense (Ness.) A. Haines = Lycopodium annotinum L. var. pungens (La Pyl.) Desv.

SPIRAEA L. (Rosaceae)

S. alba DuRoi, Narrow-leaved Meadow-sweet var. alba

$=$ S. paniculata Willd.

$=\mathrm{S}$. salicifolia of NY reports in part, not L.

var. latifolia (Ait.) Dippel, Broad-leaved

Meadow-sweet, Canada Tea

$=\mathrm{S}$. latifolia (Ait.) Borkh.

$=\mathrm{S}$. sitchensis of NY reports, not Roemer

Spiraea latifolia (Ait.) Borkh. $=$ Spiraea alba

DuRoi var. latifolia (Ait.) Dippel

Spiraea opulifolia L. = Physocarpus opulifolius

(L.) Maxim. var. opulifolius

Spiraea paniculata Willd. $=$ Spiraea alba DuRoi var. alba

Spiraea salicifolia of some NY reports in part, not L. = Spiraea alba Du Roi var. latifolia (Ait.) Dippel

Spiraea sitchensis of NY reports, not Roemer $=$ Spiraea alba Du Roi var. latifolia (Ait.) Dippel

Spiraea sorbifolia L. = Sorbaria sorbifolia (L.) A. $\mathrm{Br}$.

S. tomentosa L., Hardhack var. tomentosa

Spiraea trifoliata L. $=$ Porteranthus trifoliatus (L.) Britt.

SPIRANTHES L. Rich. (Orchidaceae)

S. casei Catl. \& Cruise, Lady's-tresses

S. cernua (L.) L. Rich., Nodding Lady's-tresses

S. lacera (Raf.) Raf., Slender Lady's-tresses var. gracilis (Bigel.) Luer

$=\mathrm{S}$. gracilis (Bigel.) Beck

Spiranthes latifolia Torr. ex Lindl. $=$ Spiranthes lucida (H. Eat.) Ames

S. lucida (H. Eat.) Ames, Shining Lady's-tresses $=$ Ibidium plantagineum Rafinesque sec. Macdonald 1990

$=$ Spiranthes latifolia Torr. ex Lindl.

$=\mathrm{S}$. plantaginea Raf.

[S. magnicamporum Shev. (Regl. M. Niag. Oldham 1999; see Larson 1995.]

S. ochroleuca (Rydb. ex Britt.) Rydb., Creamy Lady's-Tresses

$=$ S. cernua var. ochroleuca (Rydb. ex Britt.) Ames

Spiranthes plantaginea Raf. $=$ Spiranthes lucida $(H$. Eat.) Ames

S. romanzoffiana Cham., Hooded Lady's-tresses

SPIRODELA Schleid. (Lemnaceae)

S. polyrhiza (L.) Schleiden, Greater Duckweed, Giant Duckweed, Water-flaxweed $=$ Lemna polyrhiza $\mathrm{L}$.

SPOROBOLUS R. Br. (Poaceae)

Sporobolus airoides (Torr.) Torr. (excluded by Zander \& Pierce 1979)

S. asper (Michx) Kunth, Rush-grass, Dropseed *S. cryptandrus (Torr.) A. Gray, Sand Dropseed S. neglectus Nash, Small Rush-grass, Povertygrass

S. vaginiflorus (Torr. ex A. Gray) Wood, Sheathed Rush-grass, Poverty-grass $=\mathrm{S}$. vaginiflorus var. inaequalis Fern.

STACHYS L. (Lamiaceae)

Stachys aspera Michx. = Stachys hyssopifolia Michx.

Stachys hispida Pursh = Stachys tenuifolia Willd .

S. hyssopifolia Michx., Rough Hedge-nettle $=\mathrm{S}$. aspera Michx. 
= S. hyssopifolia var. ambigua A. Gray

$=$ S. tenuifolia var. aspera (Michx.) Fern.

S. palustris L., Woundwort

$=\mathrm{S}$. palustris varieties

$=\mathrm{S}$. palustris var. homotricha Fern.

S. tenuifolia Willd., Creeping Hedge-nettle

$=\mathrm{S}$. hispida Pursh

$=$ S. tenuifolia var. aspera (Michx.) Fern.

$=$ S. tenuifolia var. hispida (Pursh) Fern.

$=\mathrm{S}$. tenuifolia var. platyphylla Fern.

Stachys tenuifolia var. aspera (Michx.) Fern. = Stachys hyssopifolia Michx.

STAPHYLEA L. (Staphyleaceae)

S. trifolia L., Bladdernut

Steironema ciliatum (L.) Raf., not Baudo = Lysimachia ciliata L.

Steironema hybridum (Michx.) Raf. = Lysimachia hybrida Michx.

Steironema lanceolatum (Walt.) Gray $=$ Lysimachia lanceolata Walt. (excluded by Zander \& Pierce 1979)

Steironema quadriflorum (Sims) Hitchc. $=$ Lysimachia quadriflora Sims

STELLARIA L. (Caryophyllaceae)

Stellaria aquatica (L.) Scop. $=$ Myosoton aquaticum (L.) Moench

Stellaria biflora Pursh $=$ Moehringia lateriflora $(\mathrm{L}$. Fenzl

S. borealis Bigel., Northern Stichwort or Starwort $=\mathrm{S}$. borealis var. floribunda Fern.

$=\mathrm{S}$. borealis var. isophylla Fern.

$=\mathrm{S}$. calycantha of NY reports, not (Ledeb.)

Bong.

Stellaria calycantha of NY reports, not (Ledeb.)

Bong. = Stellaria borealis Bigel.

*S. graminea L., Lesser Stichwort

= S. graminifolia Salisb.

$=$ S. montana Raf.

Stellaria graminifolia Salisb. $=$ Stellaria graminea L.

S. longifolia Muhl. ex Willd., Long-leaved Stichwort

$=\mathrm{S}$. longipes var. laeta (Richardson) S. Wats. $=\mathrm{S}$. tenella Raf.

Stellaria longipes var. laeta (Richardson) S. Wats. $=$ Stellaria longifolia Muhl. ex Willd.

*S. media (L.) Vill., Common Chickweed
$=$ S. neglecta Weihe ex Bluff \& Fingerhut

Stellaria montana Raf. $=$ Stellaria graminea L.

Stellaria neglecta Weihe ex Bluff \& Fingerhut $=$ Stellaria media (L.) Vill.

Stellaria tenella Raf. $=$ Stellaria longifolia Muhl. ex Willd.

STREPTOPUS Michx. (Liliaceae)

S. amplexifolius (L.) DC. var. americanus Schultes, Clasping-leaved Twisted-stalk, White Mandarin, Liverberry

S. roseus Michx. (Liliaceae) Twisted-stalk, Rose Mandarin

$=\mathrm{S}$. roseus var. curvipes (Vail) Fassett

$=\mathrm{S}$. roseus var. perspectus Fassett

Strophocaulos arvensis (L.) Small $=$ Convolvulus arvensis L.

STROPHOSTYLES Ell. (Fabaceae)

Strophostyles angulosa (Ort.) Ell. = Strophostyles helvula (L.) Ell.

S. helvula (L.) Ell., Trailing Wild Bean, Trailing Fuzzy Bean

$=\mathrm{S}$. angulosa (Ort.) Ell.

$=\mathrm{S}$. helvola of authors

Struthiopteris germanica Willd. = Matteuccia struthiopteris (L.) Todaro

Stuckenia filiformis (Pers.) Börner ssp. alpinus (Blytt) Haynes, Les \& Kral = Coleogeton filiformis (Pers.) D. Les \& R. Haynes ssp. alpinus (Blytt) D. Les \& R. Haynes

Stuckenia filiformis (Pers.) Börner ssp. occidentalis (Robbins) Haynes, Les \& Kral $=$ Coleogeton filiformis (Pers.) D. Les \& R. Haynes ssp. occidentalis (Robbins) D. Les \& R. Haynes

Stuckenia pectinata (L.) Börner $=$ Coleogeton pectinatum (L.) D. Les \& R. Haynes

SUCCISELLA G. Beck (Dipsacaceae)

*S. inflexa (Kluk) Beck, Devil's-bit

$=$ Scabiosa australis Wulf.

$=$ Succisa australis (Wulf.) Reichenb.

Succisa australis (Wulf.) Reichenb. $=$ Succisella inflexa (Kluk) Beck

Svida alternifolia (L.f.) Small $=$ Cornus alternifolia L.f.

Swertia caroliniensis (Walt.) Kuntze $=$ Frasera caroliniensis Walt. 
SYMPHORICARPOS Duham. (Caprifoliaceae)

S. albus (L.) Blake, Snowberry

var. albus

$=\mathrm{S}$. racemosus var. paucifloris Robbins

$=\mathrm{S}$. racemosus Michx.

var. laevigatus (Fern.) Blake, Garden

Snowberry

*S. orbiculatus Moench, Coralberry, Indiancurrant

$=\mathrm{S}$. vulgaris Michx.

Symphoricarpos racemosus var. paucifloris

Robbins $=$ Symphoricarpos albus (L.)

Blake var. albus

Symphoricarpos racemosus Michx. =

Symphoricarpos albus var. albus (L.)

Blake

Symphoricarpos vulgaris Michx. = Symphoricarpos orbiculatus Moench

Symphyotrichum boreale (Torr. \& A. Gray) Á. \&

D. Löve $=$ Aster borealis $($ Torr. \& A. Gray) Prov.

Symphyotrichum ciliatum (Ledeb.) Nesom = Brachyactis ciliata (Ledeb.) Ledeb. ssp. angusta (Lindl.) A.G. Jones

Symphyotrichum cordifolium (L.) Nesom $=$ Aster cordifolius L.

Symphyotrichum ericoides (L.) Nesom = Aster ericoides L.

Symphyotrichum laeve (L.) A. \& D. Löve var. leave $=$ Aster laevis $\mathrm{L}$.

Symphiotrichum lanceolatum (Willd.) Nesom var. lanceolatum $=$ Aster lanceolatus Willd . var. lanceolatus

Symphyotrichum lateriflorum (L.) Á. \& D. Löve = Aster lateriflorus (L.) Britt. var. lateriflorus

Symphyotrichum lowrieanum (Porter) Nesom = Aster lowrieanum Porter

Symphyotrichum novae-angliae (L.) Nesom = Aster novae-angliae L.

Symphyotrichum novi-belgii (L.) Nesom var. crenifolium (Fern.) J. Labrecque \& L. Brouillet $=$ Aster novi-belgiae L. var. tardiflorus (L.) A. Jones

Symphyotrichum oolentangiensis (Riddell) Nesom var. oolentangiensis $=$ Aster oolentangiensis Riddell

Symphyotrichum pilosum (Willd.) Nesom var. pilosum $=$ Aster pilosus Willd. var. pilosus
Symphyotrichum praealtum (Poir.) Nesom var. praealtum $=$ Aster praealtus Poir.

Symphyotrichum prenanthoides (Muhl. ex Willd.) Nesom $=$ Aster prenanthoides Muhl. ex Willd.

Symphyotrichum puniceum (L.) Á. \& D. Löve = Aster puniceus L. var. puniceus

Symphyotrichum tradescantii (L.) Nesom $=$ Aster lateriflorus (L.) Britt. var. hirsuticaulis (Lindl. ex DC.) Porter

Symphyotrichum urophyllum (Lindl.) Nesom (the accepted name sec. NYFA July 2005) = Aster sagittifolius "auct. non Wedemeyer ex Willd."

SYMPHYTUM L. (Boraginaceae)

*S. asperum Lepechin, Rough Comfrey, Prickly Comfrey

*S. officinale L., Common Comfrey

$=$ S. officinale ssp. uliginosum (Kern.) Numan

$=\mathrm{S}$. uliginosum Kern.

SYMPLOCARPUS Salisb. ex Nutt. (Araceae)

S. foetidus (L.) Salisb. ex Nutt., Skunk-cabbage, Devil's Tobacco (Tabac du diable)

$=$ Dracontium foetidum $\mathrm{L}$.

= Spathyema foetida (L.) Raf.

Syndesmon thalictroides (L.) Hoffm. = Thalictrum thalictroides (L.) Eames \& Boivin

Syntherisma ishaemum (Schreb. ex Schweig.) Nash $=$ Digitaria ischaemum (Schreb. ex Schweig.) Schreb. ex Muhl.

Syntherisma sanguinalis (L.) Dulac $=$ Digitaria sanguinalis (L.) Scop.

SYRINGA L. (Oleaceae)

*S. vulgaris L., Lilac, Purple Lilac, White Lilac

TAENIDIA (Torr. \& A. Gray) Drude (Apiaceae)

T. integerrima (L.) Drude, Yellow Pimpernel $=$ Zizia integerrima DC.

Tagetes papposa Vent. $=$ Dyssodia papposa $($ Vent. $)$ Hitchc.

TANACETUM L. (Asteraceae)

Tanacetum balsamita L. = Balsamita major Desf.

*T. parthenium (L.) Schultz, Feverfew $=$ Chrysanthemum parthenium (L.) Bernh. 
$=$ Leucodon parthenium Godron. of Gray's

Manual, 5th ed. 1867 p. 266

$=$ Matricaria parthenium L. according to

House 1924 p. 735

*T. vulgare L., Common Tansy, Golden-buttons

$=$ Chrysanthemum vulgare (L.) Bernh.

$=$ Tanacetum vulgare var. crispum DC.

TARAXACUM Weber ex Wiggers (Asteraceae)

Taraxacum dens-leonis Desf. $=$ Taraxacum officinale Weber ex Wiggers

Taraxacum erythrospermum Andrz. ex Besser = Taraxacum laevigatum (Willd.) DC.

*T. laevigatum (Wild.) DC., Red-seeded

Dandelion

$=$ Leontodon laevigatus Willd.

$=\mathrm{T}$. erythrospermum Andrz. ex Besser

*T. officinale Weber ex Wiggers, Common Dandelion

$=$ Leontodon taraxacum L.

$=$ Taraxacum dens-leonis Desf.

$=\mathrm{T}$. officinale ssp. vulgare (Lam.) Schinz \& Keller

TAXODIUM Rich. (Taxodiaceae)

*T. distichum (L.) Rich., Bald Cypress

TAXUS L. (Taxaceae)

Taxus baccata var. canadensis (Marsh.) Gray = Taxus canadensis Marsh.

T. canadensis Marsh., Ground-hemlock, American Yew

$=\mathrm{T}$. baccata var. canadensis (Marsh.) A.

Gray

TEUCRIUM L. (Lamiaceae)

T. canadense L., Hairy Germander

var. boreale (Bickn.) Shinners, Northern

Germander

$=\mathrm{T}$. occidentale Gray

$=\mathrm{T}$. canadense var. occidentale (Gray)

McClint. \& Eping

var. canadense, Seaside Germander

$=\mathrm{T}$. canadense var. littorale (Bickn.) Fern.

$=\mathrm{T}$. canadense var. virginicum (L.) Eaton

$=\mathrm{T}$. littorale Bickn.

Thalesia uniflora (L.) Britt. = Orobanche uniflora

L.
THALICTRUM L. (Ranunculaceae)

Thalictrum anemonoides Michx. $=$ Thalictrum thalictroides (L.) Eames \& Boivin

Thalictrum canadense of NY reports, not Mill. = Thalictrum pubescens Pursh

Thalictrum cornutii (of authors) $=$ Thalictrum pubescens Pursh

T. dasycarpum Fisch. \& Ave-Lall., Purple Meadow-rue $=\mathrm{T}$. purpurascens Pursh

T. dioicum L., Early Meadow-rue

T. pubescens Pursh, Late Meadow-rue $=\mathrm{T}$. canadense of NY reports, not Mill. $=\mathrm{T}$. cornutii (of authors)

$=\mathrm{T}$. polygamum Muhl.

Thalictrum purpurascens Pursh $=$ Thalictrum dasycarpum Fisch. \& Ave-Lall.

T. revolutum DC., Waxy Meadow-rue

$=\mathrm{T}$. canadensis Mill. var. hebecarpum (Fern.) House

$=\mathrm{T}$. dasycarpum of some early NY reports, not Fisch. \& Ave-Lall.

$=\mathrm{T}$. purpurascens of early NY reports, not Pursh.

T. thalictroides (L.) Eames \& Boivin, Rueanemone, Woods-potato

$=$ Anemone thalictroides L.

$=$ Anemonella thalictroides (L.) Spach

$=$ Syndesmon thalictroides (L.) Hoffm.

$=$ Thalictrum anemonoides Michx.

THASPIUM Nutt. (Apiaceae)

Thaspium aureum (of authors) $=$ Thaspium trifoliatum (L.) A. Gray var. aureum (L.) Britt.

Thaspium aureum Nutt.: see discussion under Thaspium trifolium var. aureum.

T. barbinode (Michx) Nutt., Hairy-jointed Meadow-parsnip

T. trifoliatum (L.) A. Gray, Purple Meadowparsnip

var. aureum (L.) Britt.

Following House (1924), Thaspium aureum Nutt., l.c., is only a synonym of Thaspium trifoliatum, s.l., not of Zizia aurea. Thaspium trifoliatum var. trifoliatum (a southern plant) is not known for (never occurred in) New York State nor Ontario, but the variety aureum (L.) Britt. is 
reported for both areas. It is considered extirpated in New York State (Mitchell \& Tucker 1997) and hence, probably extirpated from the Niagara Frontier region.

Thaspium trifoliatum (L.) A. Gray var. apterum A. Gray $=$ Zizia aptera (A. Gray) Fern.

THEL YPTERIS Schmid. (Thelypteridaceae) Thelypteris clintoniana (D. C. Eaton ex A. Gray) House $=$ Dryopteris clintoniana $($ D. $C$. Eaton ex A. Gray) Dowell

Thelypteris cristata (L.) Nieuwl. = Dryopteris cristata (L.) A. Gray

Thelypteris cristata var. clintoniana (D.C. Eat.) Weatherby $=$ Dryopteris clintoniana (D.C. Eat. ex A. Gray) Dowell

Thelypteris cristata var. clintoniana $\times \mathrm{T}$. spinulosa var. intermedia of Zenkert $(1934)=$ [Dryopteris clintoniana $\times$ intermedia $=\mathrm{D}$. $x$ dowellii (Farw.) Wherry]

Thelypteris dilatata $($ Hoffm. $)$ House $=$ Dryopteris campyloptera (Kunze) Clarkson

Thelypteris dilatata var. americana (Fisch.) House $=$ Dryopteris campyloptera (Kunze) Clarkson

Thelypteris dryopteris (L.) Slosson = Gymnocarpium dryopteris (L.) Newm.

Thelypteris goldiana (Hook.) Nieuwl. = Dryopteris goldiana (Hook. ex Goldie) Nieuwl.

Thelypteris hexagonoptera (Michx.) Weath. = Phegopteris hexagonoptera (Michx.) Fée

Thelypteris intermedia (Muhl. ex Willd.) House = Dryopteris intermedia (Muhl. ex Willd.) A. Gray

Thelypteris marginalis (L.) Nieuwl. = Dryopteris marginalis (L.) A. Gray

T. noveboracensis (L.) Nieuwl., New York Fern $=$ Dryopteris noveboracensis (L.) A. Gray $=$ Aspidium noveboracense (L.) Sw.

T. palustris Schott, Marsh-fern

$=$ Acrostichum thelypteris L.

$=$ Dryopteris thelypteris (L.) Sw., not A. Gray

$=$ Aspidium thelypteris (L.) Sw.

$=$ Thelypteris palustris var. pubescens (Lawson) var. pubescens (Lawson) Fern.

Thelypteris phegopteris (L.) Slosson $=$ Phegopteris connectilis (Michx.) Watt
Thelypteris spinulosa (O.F. Muell.) Nieuwl. = Dryopteris carthusiana (Vill.) Fuchs

Thelypteris spinulosa var. intermedia (Muhl.) Weatherby $=$ Dryopteris intermedia (Muhl. ex Willd.) A. Gray

Thesium umbellatum L. = Comandra umbellata (L.) Nutt.

THLASPI L. (Brassicaceae)

*T. arvense L., Field Penny-cress, Fanweed, French-weed, Mithridate Mustard

Thlaspi bursa-pastoris L. $=$ Capsella bursa-pastoris (L.) Medik.

Thlaspi campestre L. = Lepidium campestre (L.) R. Br. ex Ait.

THUJA L. (Cupressaceae)

T. occidentalis L., Arbor Vitae, Northern White Cedar

THYMUS L. (Lamiaceae)

*T. pulegioides L., Creeping Thyme, Mother-ofthyme, Lemon-thyme

$=\mathrm{T}$. serpyllum of American authors, not L.

Thymus serpyllum of American authors, not L. = Thymus pulegioides L.

TIARELLA L. (Saxifragaceae)

T. cordifolia L., Foam-flower, False Miterwort

TILIA L. (Tiliaceae)

T. americana L., Basswood, American Linden, Whitewood

var. americana

$=\mathrm{T}$. americana var. neglecta (Spach)

Fosberg.

$=\mathrm{T}$. glabra Vent.

$=\mathrm{T}$. neglecta Spach

var. heterophylla (Vent.) Loud., White Basswood

$=\mathrm{T}$. heterophylla Vent.

$=\mathrm{T}$. heterophylla var. michauxii (Nutt.) Sarg.

$=\mathrm{T}$. michauxii Nutt.

*T. cordata Mill., Small-leaved Linden, European Linden, Heart-leaf Linden

$=\mathrm{T}$. europaea L. in part

$=\mathrm{T}$. parviflora Ehrh.

$=\mathrm{T}$. ulmifolia Scop.

Tilia europaea L . in part $=$ Tilia cordata Mill. 
Tilia glabra Vent. $=$ Tilia americana L. var. americana

Tilia heterophylla Vent. $=$ Tilia americana var . heterophylla (Vent.) Loud.

Tilia heterophylla var. michauxii (Nutt.) Sarg. = Tilia americana var. heterophylla (Vent.) Loud.

Tilia michauxii Nutt. $=$ Tilia americana var. heterophylla (Vent.) Loud.

Tilia neglecta Spach = Tilia americana L. var. americana

Tilia parviflora Ehrh. $=$ Tilia cordata Mill.

Tilia ulmifolia Scop. = Tilia cordata Mill.

Tissa marina (L.) Britt. = Spergularia salina J. \& C. Presl

TOFIELDIA Huds. (Liliaceae)

T. glutinosa (Michx.) Pers., False Asphodel

$=\mathrm{T}$. racemosa of NY reports, not (Walt.) BSP.

$=$ Triantha glutinosa (Michx.) Baker

Tofieldia racemosa of NY reports, not (Walt.) BSP. $=$ Tofieldia glutinosa (Michx.) Pers.

TORILIS Adans. (Apiaceae)

Torilis anthriscus (L.) Gmel. $=$ Torilis japonica (Houtt) DC.

*T. japonica (Houtt) DC., Hedge-parsley

$=\mathrm{T}$. anthriscus (L.) Gmel.

$=\mathrm{T}$. arvensis of authors, not (Huds.) Link

$=\mathrm{T}$. nodosa of reports, not (L.) Gaertn.

TORREYOCHLOA Church (Poaceae)

T. pallida (Torr.) Church, Pale Mannagrass

var. pallida

$=$ Puccinellia pallida (Torr.) Clausen in part

$=$ Glyceria pallida (Torr.) Trin.

var. fernaldii (Hitchc.) Church

$=$ Glyceria fernaldii (Hitchc.) St. John

$=$ Panicularia fernaldii (Hitchc.) House

$=$ Puccinellia pallida (Torr.) Clausen in part

Tovara virginiana (L.) Raf. $=$ Polygonum virginianum $\mathrm{L}$.

TOXICODENDRON Mill. (Anacardiaceae)

T. radicans (L.) Kuntze, Poison-ivy, Climbing Poison-ivy

ssp. radicans

$=$ Rhus radicans $\mathrm{L}$.
$=$ Rhus toxicodendron of NY reports, not $\mathrm{L}$.

T. rydbergii (Small ex Rydb.) Greene, Giant Poison-ivy, Poison-oak, Creeping Poisonivy

$=\mathrm{R}$. radicans var. rydbergii (Small ex Rydb.) Rehd.

$=\mathrm{R}$. radicans var. vulgaris (Michx.) DC. in part

T. vernix (L.) Kuntze, Poison-sumac

$=$ Rhus venenata DC.

$=$ Rhus vernix $\mathrm{L}$.

Toxylon pomiferum Raf. ex Sarg. = Maclura pomifera (Raf. ex Sarg.) Schneid.

Tracaulon arifolium (L.) Small $=$ Polygonum arifolium $\mathrm{L}$.

Tracaulon sagittatum (L.) Small = Polygonum sagittatum L.

TRADESCANTIA L. (Commelinaceae)

*T. virginiana L., Spiderword, Widow's Tears

TRAGOPOGON L. (Asteraceae)

*T. dubius Scop., Goat's Beard

$=\mathrm{T}$. dubius var. major (Jacq.) Voll.

$=\mathrm{T}$. major Jacq.

Tragopogon major Jacq. $=$ Tragopogon dubius Scop.

*T. porrifolius L., Salsify, Oyster-plant, Vegetable-oyster

$* \mathbf{T}$. porrifolius $\times$ pratensis $=\mathbf{T}$. neohybridus Farw., Goat's-beard

*T. pratensis L., Yellow Goat's-beard

TRIADENUM Raf. (Clusiaceae)

T. fraseri (Spach) Gleason, Marsh St. John's Work, Pink-flowered St. John's-wort

= Hypericum virginicum var. fraseri (Spach) Fern.

T. virginicum (L.) Raf., Marsh St. John's-wort, Pink-flowered St. John's-wort

$=$ Hypericum virginicum $\mathrm{L}$.

Triantha glutinosa $($ Michx. $)$ Baker $=$ Tofieldia glutinosa (Michx.) Pers.

[Trichomanes intricata Farrar; "... known in NY by its minute gametophyte generation only," Mitchell and Tucker (1997). (As Trichomanes sp. "gametophytes only)" a post-1980 specimen exists for Cattaraugus Co. (NYFA 1990).] 
Trichophorum alpinum (L.) Pers. = Scirpus hudsonianus (Michx.) Fern.

Trichophorum caespitosum (L.) Hartm. = Scirpus caespitosus L.

Trichophorum clintonii (A. Gray) S. G. Sm. = Scirpus clintonii A. Gray

Trichophorum planifolium (Spreng.) Palla $=$ Scirpus verecundus Fern.

TRICHOSTEMA L. (Lamiaceae)

T. brachiatum L., False Pennyroyal $=$ Isanthus brachiatus (L.) BSP.

$=\mathrm{I}$. coeruleus Michx.

Triclinium odoratum Raf. $=$ Sanicula odorata (Raf.) Pryer \& Phillippe

Tricuspis flava (L.) Hubbard = Triodia flava (L.) Smyth

Tricuspis purpurea (Walt.) Gray $=$ Triplasis purpurea (Walt.) Chapm.

Tricuspis seslerioides Torrey $=$ Tridens flavus $(\mathrm{L}$. Hitchc.

TRIDENS R. \& S. (Poaceae)

T. flavus (L.) Hitchc., Tall Redtop

$=$ Triodea flava $($ L.) Smyth

$=$ Tricuspis flava (L.) Hubbard

$=\mathrm{T}$. seslerioides Torrey of Gray's Manual ed 5

TRIENTALIS L. (Primulaceae)

Trientalis americana Pursh $=$ Trientalis borealis Raf.

T. borealis Raf., Starflower, Chickweed, Wintergreen Star-of-Seven

$=\mathrm{T}$. americana Pursh

$=\mathrm{T}$. borealis var. tenuifolia House

$=\mathrm{T}$. europea of American authors, not L.

$=\mathrm{T}$. latifolia of NY reports, not Hooker

Trientalis europea of American authors, not L. = Trientalis borealis Raf.

Trientalis latifolia of NY reports, not Hooker $=$ Trientalis borealis Raf.

TRIFOLIUM L. (Fabaceae)

Trifolium agrarium L., a nomen ambiguum $=$ Trifolium aureum Pollich

*T. arvense L., Rabbit-foot Clover, Old Field- or Stone-clover

*T. aureum Pollich, Yellow Hop-clover
$=$ Trifolium agrarium L., a nomen ambiguum

*T. campestre Schreber, Hop-clover, Low Hopclover, Shamrock, Cow-clover

$=\mathrm{T}$. procumbens L. of some authors, a nomen ambiguum (Mitchell \& Tucker 1997).

*T. dubium Sibth., Little Hop-hlover, Least Hopclover

$=\mathrm{T}$. minus $\mathrm{Sm}$.

$=\mathrm{T}$. procumbens L., of some authors, a nomen ambiguum

*T. hybridum L., Alsike Clover

$=\mathrm{T}$. hybridum var. elegans (Savi) Boiss.

$=\mathrm{T}$. reflexum of some NY reports, not $\mathrm{L}$. (1997).

Trifolium hybridum var. elegans (Savi) Boiss. = Trifolium hybridum L.

*T. incarnatum L., Crimson Clover, Scarlet Clover, Italian Clover

Trifolium meliloti-officinalis of authors, not L. = Melilotus officinalis (L.) Pallas

Trifolium minus Sm. $=$ Trifolium dubium Sibth .

Trifolium officinalis L. $=$ Melilotus officinalis (L.) Pallas

*T. pratense L., Red Clover $=\mathrm{T}$. pratense var. sativum (Mitt.) Schreb.

Trifolium procumbens L. of some authors, a nomen ambiguum (Mitchell \& Tucker 1997) $=$ Trifolium campestre Schreber.

Trifolium procumbens L., of some authors, a nomen ambiguum (Mitchell \& Tucker 1997) $=$ Trifolium dubium Sibth.

T. reflexum L., Buffalo-clover $=\mathrm{T}$. reflexum var. glabrum Lojac

Trifolium reflexum of some NY reports, not L. $=$ Trifolium hybridum L.

Trifolium reflexum var. glabrum Lojac $=$ Trifolium reflexum L.

*T. repens L., Creeping White Clover, Lawnclover, Dutch-clover

TRIGLOCHIN L. (Juncaginaceae)

Triglochin elata Nutt. $=$ Triglochin maritimum L.

T. maritimum L., Seaside Arrow-grass

$=\mathrm{T}$. elata Nutt.

$=\mathrm{T}$. maritimum var. elatum (Nutt.) Gray

T. palustre L., Marsh Arrow-grass

$=\mathrm{T}$. palustris of authors 
TRILLIUM L. (Liliaceae)

T. cernuum L., Nodding-Trillium var. cernuum

T. erectum L., Red Trillium, Stinking Benjamin $=\mathrm{T}$. erectum var. album (Michx.) Pursh

Trillium erythrocarpum Michx. $=$ Trillium undulatum Willd.

T. flexipes Raf., Nodding Trillium

$=\mathrm{T}$. erectum var. blandum Jenn.

$=\mathrm{T}$. erectum var. declinatum of authors, not A. Gray

$=\mathrm{T}$. gleasonii Fern .

T. grandiflorum (Michx) Salisb., White Trillium, Wakerobin

T. sessile L., Toadshade

T. undulatum Willd., Painted Trillium

$=\mathrm{T}$. erythrocarpum Michx.

TRIODANIS Raf. (Campanulaceae)

T. perfoliata (L.) Nieuwl., Venus' Looking-glass var. perfoliata

$=$ Specularia perfoliata (L.) A. DC.

Triodia flava (L.) Smyth = Tridens flavus (L.)

Hitchc.

Trionum trionum $(\mathrm{L}$.$) Wooten \&$ Standl. $=$ Hibiscus trionum L.

TRIOSTEUM L. (Caprifoliaceae)

T. aurantiacum Bickn., Orange Horse-gentian, Wild Coffee

$=\mathrm{T}$. perfoliatum var. aurantiacum (Bickn.) Wieg.

$=\mathrm{T}$. perfoliatum var. glaucescens (Wieg.)

Wieg.

Triosteum aurantiacum var. glaucescens (Wieg.)

Wieg. $=$ Triosteum aurantiacum Bickn.

T. perfoliatum L., Horse-gentian, Tinker's Weed, Wild Coffee, Feverwort

Triosteum perfoliatum var. aurantiacum (Bickn.)

Wieg. $=$ Triosteum aurantiacum Bickn.

Triosteum perfoliatum var. glaucescens (Wieg.)

Wieg. $=$ Triosteum aurantiacum Bickn.

TRIPHORA Nutt. (Orchidaceae)

T. trianthophora (Sw.) Rydb., Three-birds

Orchid, Nodding

$=$ Pogonia trianthophora $(\mathrm{Sw}$.$) BSP.$

TRIPLASIS Beauv. (Poaceae)
T. purpurea (Walt.) Chapm., Purple Sand-grass $=$ Tricuspis purpurea (Walt.) Gray

Tripleurospermum maritimum ssp. inodorum (L.) Hyl. = Matricaria perforata Merat

Tripleurospermum perforatum (Merat) $\mathrm{M}$. Lainz = Matricaria perforata Merat

TRIPSACUM L. (Poaceae)

T. dactyloides (L.) L., Gamma-grass

Trisetum pensylvanicum (L.) Beauv. ex R. \& S. = Sphenopholis pensylvanica (L.) Hitchc.

TRITICUM L. (Poaceae)

*T. aestivum L., Wheat

Triticum caninum L. = Elymus trachycaulus (Link) Gould ex Shinners ssp. subsecundus (Link) Barkworth \& D. Dewey

Triticum repens L. $=$ Elytrigia repens (L.) Nevski

Triticum repens L. var. nemorale Anderss. = Elytrigia repens (L.) Nevski

TROLLIUS L. (Ranunculaceae)

T. laxus Salisb., American Globeflower, Spreading Globeflower

TSUGA Carrière (Pinaceae)

T. canadensis (L.) Carr., Eastern Hemlock $=$ Abies canadensis Michx.

Tulotis herbiola (R. Br.) Raf. = Platanthera flava (L.) Lindl. var. herbiola (R. Br.) Luer Turritis glabra L. = Arabis glabra (L.) Bernh. Turritis stricta Graham, not Arabis stricta Huds. according to House, 1924 p.365 = Arabis. drummondii A. Gray

TUSSILAGO L. (Asteraceae)

*T. farfara L., Coltsfoot

TYPHA L. (Typhaceae)

T. angustifolia L., Narrow-leaved Cat-tail

T. latifolia L., Broad-leaf Cattail

ULMUS L. (Ulmceae)

U. americana L., American Elm, White Elm

Ulmus campestris L. of authors in part $=$ Ulmus minor Mill.

Ulmus fulva Michx. = Ulmus rubra Muhl.

*U. minor Mill., English Elm $=\mathrm{U}$. campestris L. of authors in part 
$=\mathrm{U}$. procera Salisb.

= U. scabra Mill.

Ulmus procera Salisb. $=$ Ulmus minor Mill.

*U. pumila L., Siberian Elm, Dwarf Elm

Ulmus racemosa (of authors) $=$ Ulmus thomasii

Sarg.

U. rubra Muhl., Slippery Elm, Red Elm $=$ U. fulva Michx.

Ulmus scabra Mill. = Ulmus minor Mill.

U. thomasii Sarg., Rock Elm $=$ Ulmus racemosa (of authors)

Unifolium canadense (Desf.) Greene = Maianthemum canadense Desf.

Unifolium canadense (Desf.) Greene var. interior

(Fern.) House $=$ Maianthemum canadense

Desf.

URTICA L. (Urticaceae)

Urtica canadensis L. = Laportea canadensis (L.) Wedd.

U. dioica L., Stinging Nettle, Great Nettle *ssp. dioica

ssp. gracilis (Ait.) Selander

$=$ U. dioica var. gracilis (Ait.) Taylor \&

$\mathrm{McBr}$.

$=$ U. dioica var. procera (Muhl.) Wedd.

$=\mathrm{U}$. gracilis Ait.

$=\mathrm{U}$. procera Muhl.

Urtica dioica var. gracilis (Ait.) Taylor $\& \mathrm{McBr} .=$ Urtica dioica ssp. gracilis (Ait.) Selander

Urtica divaricata L. = Laportea canadensis (L.)

Wedd.

Urtica gracilis Ait. = Urtica dioica ssp. gracilis (Ait.) Selander

Urtica procera Muhl. ex Willd. = Urtica dioica ssp. gracilis (Ait.) Selander

*U. urens L., Burning Nettle, Dog-nettle

Urticastrum divaricatum (L.) Kuntze = Laportea canadensis (L.) Wedd.

UTRICULARIA L. (Lentibulariaceae)

Utricularia clandestina Nutt. ex A. Gray = Utricularia geminiscapa Benj.

U. cornuta Michx., Horned Bladderwort

U. geminiscapa Benj., Hidden-fruited Bladderwort $=\mathrm{U}$. clandestina Nutt. ex A. Gray

U. intermedia Hayne, Flat-leaved Bladderwort, Milfoil Bladderwort

U. macrorhiza LeConte, Great Bladderwort
$=\mathrm{U}$. vulgaris of American authors, not L.

$=$ U. vulgaris var. americana Gray

U. minor L., Lesser Bladderwort

Utricularia vulgaris of American authors, not L. = Utricularia macrorhiza LeConte

UVULARIA L. (Liliaceae)

U. grandiflora Sm., Large-Flowered Bellwort

U. perfoliata L., Perfoliate Bellwort, Strawbell

U. sessilifolia L., Sessile-leaved Bellwort, Wildoats, Little Merrybells

VACCARIA Medik. (Caryophyllaceae)

*V. hispanica (Mill.) Raush., Cow-herb, Cockle

= Saponaria hispanica Mill.

= S. vaccaria L.

= Vaccaria pyramidata Medik.

=V. segetalis (Neck.) Garcke ex Aschers.

$=\mathrm{V}$. vaccaria $(\mathrm{L}$.$) Britt.$

$=\mathrm{V}$. vulgaris Host

Vaccaria pyramidata Medik. $=$ Vaccaria hispanica (Mill.) Raush.

Vaccaria segetalis (Neck.) Garcke ex Aschers. = Vaccaria hispanica (Mill.) Raush.

Vaccaria vaccaria (L.) Britt. $=$ Vaccaria hispanica (Mill.) Raush.

Vaccaria vulgaris Host $=$ Vaccaria hispanica (Mill.) Raush.

VACCINIUM L. (Ericaceae)

V. angustifolium Ait., Lowbush Blueberry, Sweet-hurts

$=\mathrm{V}$. angustifolium var. hypolasium Fern.

$=\mathrm{V}$. angustifolium var. laevifolia House

$=\mathrm{V}$. angustifolium var. nigrum (Wood) Dole

$=\mathrm{V}$. brittonii Porter ex Bickn.

$=\mathrm{V}$. lamarckii Camp

$=\mathrm{V}$. nigrum (Wood) Britt.

$=\mathrm{V}$. pensilvanicum Lam.

Vaccinium angustifolium Ait. in part. = Vaccinium boreale Hall \& Hald.

V. boreale Hall \& Hald., High-mountain Blueberry

$=\mathrm{V}$. angustifolium Ait. in part.

Pre-1980 specimens exist for Genesee Co. (NYFA 1990).

Vaccinium angustifolium var. myrtilloides (Michx.) House $=$ Vaccinium myrtilloides Michx. 
Vaccinium atrococcum (A. Gray) Heller = Vaccinium corymbosum L.

Vaccinium brittonii Porter ex Bickn. $=$ Vaccinium angustifolium Ait.

Vaccinium caesariense Mackz. $=$ Vaccinium corymbosum L.

Vaccinium canadensis Kalm = Vaccinium myrtilloides Michx.

V. corymbosum L., Black Highbush-blueberry, Swamp-blueberry, Whortleberry

$=\mathrm{V}$. atrococcum (Gray) Heller

$=\mathrm{V}$. caesariense Mackz.

$=\mathrm{V}$. corymbosum varieties

$=\mathrm{V}$. marianum $\mathrm{S}$. Wats.

Vaccinium dobbinii Burnh. $=$ Vaccinium pallidum Ait.

Vaccinium frondosum L. $=$ Gaylussacia frondosa (L.) Torr. \& A. Gray ex Torr.

Vaccinium hispidulum Marsh. $=$ Vaccinium macrocarpon Ait.

Vaccinium lamarckii Camp = Vaccinium angustifolium Ait.

V. macrocarpon Ait., Large Cranberry, Cranberry

$=$ Oxycoccus macrocarpus (Ait.) Pursh

$=$ Vaccinium hispidulum Marsh.

Vaccinium marianum S. Wats. $=$ Vaccinium corymbosum L.

V. myrtilloides Michx., Velvet-leaf Blueberry

$=\mathrm{V}$. angustifolium var. myrtilloides (Michx.) House

$=\mathrm{V}$. canadense Kalm ex Richards .

$=\mathrm{V}$. pensylvanicum var. myrtilloides (Michx.) Fern.

Vaccinium neglectum $($ Small $)$ Fern. $=$ Vaccinium stamineum L.

Vaccinium nigrum $($ Wood $)$ Britt. $=$ Vaccinium angustifolium Ait.

V. oxycoccos L., Small Cranberry

$=\mathrm{V}$. oxycoccus of authors

$=\mathrm{V}$. oxycoccus var. intermedium A. Gray

$=\mathrm{V}$. oxycoccus var. ovalifolium Michx.

V. pallidum Ait., Blueberry, Sugar Huckleberry, Low Bilberry, Early Sweet Bilberry, Low or Sweet Blueberry

$=\mathrm{V}$. dobbinii Burnh.

$=\mathrm{V}$. vacillans Kalm ex Torr.

$=\mathrm{V}$. vacillans var. crinitum Fern .

Vaccinium pensylvanicum (of authors) $=$ Vaccinium angustifolium Ait.
Vaccinium pensylvanicum var. myrtilloides (Michx.) Fern. $=$ Vaccinium myrtilloides Michx.

Vaccinium pensylvanicum var. nigrum Wood $=$ Vaccinium angustifolium Ait.

V. stamineum L., Deerberry, Squaw-huckleberry $=\mathrm{V}$. neglectum (Small) Fern.

Vaccinium vacillans Kalm ex Torr. $=$ Vaccinium pallidum Ait.

Vagnera stellata (L.) Morong = Maianthemum stellatum L.

Vagnera trifolia (L.) Morong = Maianthemum trifolium L.

VALERIANA L. (Valerianaceae)

Valeriana dioica (of authors) $=$ Valeriana sitchensis Bong.

*V. officinalis L., Garden Valerian, Garden Heliotrope

V. sitchensis Bong., Swamp- or Marsh-valerian $=$ V. uliginosa (Torr. \& A. Gray) Rydb.

$=\mathrm{V}$. sitchensis Bong. ssp. uliginosa (Torr. \&

A. Gray) Meyer

$=\mathrm{V}$. sylvatica Banks

$=\mathrm{V}$. dioica (of authors)

Valeriana sylvatica Banks $=$ Valeriana sitchensis Bong.

Valeriana uliginosa (Torr. \& A. Gray) Rydb. = Valeriana sitchensis Bong.

VALERIANELLA Mill. (Valerianaceae)

V. chenopodifolia (Pursh) DC., Goosefoot Cornsalad

$=$ Fedia fagopyrum Torr. \& A. Gray

$=$ Valerianella radiata of NY reports in part, not (L.) Dufr.

*V. locusta (L.) Laterr., European Corn-salad, Lamb's Lettuce

= Fedia olitoria (L.) Vahl

$=$ Valerianella olitoria (L.) Poll.

Valerianella olitoria (L.) Poll. $=$ Valeriana locusta (L.) Laterr.

Valerianella radiata of NY reports in part, not (L.) Dufr. $=$ Valerianella chenopodifolia (Pursh) DC.

VALLISNERIA L. (Hydrocharitaceae)

V. americana Michx., Eel-grass, Tapegrass, Wild Celery 
$=\mathrm{V}$. spiralis (of American authors)

Vallisneria spiralis (of American authors) $=$ Vallisneria americana Michx.

VERATRUM L. (Liliaceae)

V. viride Ait., False Hellebore White Hellebore, Indian-poke, Devil's Tobacco (Tabac du Diable)

VERBASCUM L. (Scrophulariceae)

*V. blattaria L., Moth Mullein

*V. phlomoides L., Clasping-leaved Mullein

*V. thapsus L., Common Mullein, Mule's-ear, Flannel-plant, Devil's Tobacco

VERBENA L. (Verbenaceae)

Verbena angustifolia Michx. $=$ Verbena simplex Lehm.

*V. bracteata Lag.\& Rodr., Large-bracted Vervain, Prostrate Vervian or Verbena $=\mathrm{V}$. bracteosa Michx.

Verbena bracteosa Michx. $=$ Verbena bracteata Lag. \& Rodr.

V. hastata L., Blue Vervain, Blue Verbena, Simpler's-joy

V. simplex Lehm., Narrow-leaved Vervain, Verbena $=\mathrm{V}$. angustifolia Michx.

*V. stricta Vent., Hoary Vervain

V. urticifolia L., White Vervain var. leiocarpa Perry \& Fern. var. urticifolia

VERBESINA L. (Asteraceae)

*V. alternifolia (L.) Britt., Wingstem $=$ Actinomeris alternifolia (L.) DC.

$=$ Coreopsis alternifolius L.

VERNONIA Schreb. (Asteraceae)

Vernonia altissima Nutt. $=$ Vernonia gigantea

(Walt.) Trel ex Brann. \& Cov.

V. gigantea (Walt.) Trel. ex Brann. \& Cov., Tall or Giant Ironweed

$=\mathrm{V}$. altissima Nutt.

V. noveboracensis (L.) Michx., New York Ironweed

VERONICA L. (Scrophulariaceae)

*V. agrestis L., Field-speedwell
V. americana (Raf.) Schwein. ex Benth., American Brooklime, American Speedwell

Veronica anagallis L. = Veronica anagallisaquatica L.

(*?) V. anagallis-aquatica L., Water-speedwell, Brook- or Water- pimpernell

$=\mathrm{V}$. anagallis $\mathrm{L}$.

$=\mathrm{V}$. brittonii Porter ex Pennel

$=\mathrm{V}$. catenata Pennell

$=\mathrm{V}$. comosa Richter

$=\mathrm{V}$. connata of authors, not Raf.

$=\mathrm{V}$. cymbalaria of NY reports, not Bodard

$=\mathrm{V}$. salina of authors, not Shur

*V. arvensis L., Corn-speedwell

Veronica brittonii Porter ex Pennel $=$ Veronica anagallis-aquatica $\mathrm{L}$.

Veronica catenata Pennell $=$ Veronica anagallisaquatica $\mathrm{L}$.

*V. chamaedrys L., Bird's-eye Speedwell

Veronica comosa Richter $=$ Veronica anagallisaquatica $\mathrm{L}$.

Veronica cymbalaria of NY reports, not Bodard $=$ $\mathrm{V}$. anagallis-aquatica $\mathrm{L}$.

*V. filiformis Sm., Slender Speedwell, Bird's Eye

*V. hederifolia L., Ivy-leaf Speedwell

*V. longifolia L., Long-leaved Speedwell $=\mathrm{V}$. maritima L.

Veronica maritima L. $=$ Veronica longifolia L .

*V. officinalis L., Common Speedwell, Gypsyweed

V. peregrina L., Purslane-speedwell, Neckweed ssp. peregrina

ssp. xalapensis (Kunth) St. John \& Warren

$=\mathrm{V}$. xalapensis Kunth

$=\mathrm{V}$. peregrina var. xalapensis (Kunth) St. John \& Warren

*V. persica Poir., Persian Speedwell, Bird's-eye Speedwell $=\mathrm{V}$. tournefortii (of authors)

= V. buxbaumii Ten., not of F. W. Schmidt

Veronica salina (of authors), not Shur $=$ Veronica anagallis-aquatica $\mathrm{L}$.

V. scutellata L., Marsh-speedwell $=$ V. scutellata var. villosa Schum.

*V. serpyllifolia L., Thyme-leaved Speedwell

*ssp. humifusa (Dicks. ex With.) Syme $=\mathrm{V}$. humifusa Dickson ex With. [M\&V] $=\mathrm{V}$. serpyllifolia var. humifusa (Dicks. ex With.) Vahl 
$=\mathrm{V}$. tenella All.

*ssp. serpyllifolia

V. spicata L., Speedwell

Veronica tournefortii (of authors) $=$ Veronica persica Poir.

Veronica virginica $\mathrm{L} .=$ Veronicastrum virginicum (L.) Farw.

Veronica xalapensis Kunth $=$ Veronica peregrina L. ssp. xalapensis (Kunth) Pennell

VERONICASTRUM Heister ex Fabr. (Scrophulariaceae)

V. virginium (L.) Farw., Culver's-root $=$ Veronica virginica L.

VIBURNUM L. (Caprifoliaceae)

V. acerifolium L., Maple-leaved Viburnum, Arrowwood, Dockmackie, Purple-leaf Viburnum

Viburnum affine Bush = Viburnum rafinesquianum Schultes

Viburnum affine var. hypomalacum Blake $=$ Viburnum rafinesquianum Schultes

Viburnum alnifolium Marsh. = Viburnum lantanoides Michx.

Viburnum cassinoides L. = Viburnum nudum var. cassinoides (L.) Torr. \& A. Gray

V. dentatum L., Arrow-wood var. lucidum Ait.

$=\mathrm{V}$. recognitum Fern.

Viburnum eradiatum (Oakes) House $=$ Viburnum opulus L. var. americanum Ait.

*V. lantana L., Wayfaring Tree, Twistwood, Lantata

V. lantanoides Michx., Hobblebush, Witchhobble, Moosewood, Tanglewood $=\mathrm{V}$. alnifolium Marsh.

V. lentago L., Nannyberry, Sheepberry, Sweetberry, Black Haw, Cowberry, Wild Raisin, Wild Tea.

$=\mathrm{V}$. lentago var. sphaerocarpum A. Gray

V. nudum L., Withe-rod, Possom-haw, Swamphaw, Withe-rod, Appalachian Tea, Teaberry, Wild Raisin

var. cassinoides (L.) Torr. \& A. Gray

$=\mathrm{V}$. cassinoides $\mathrm{L}$.

$=\mathrm{V}$. nudum as to $\mathrm{ON}$ reports, not of $\mathrm{L}$.

var. nudum, Smooth Withe-rod, Possom-haw, Swamp-haw, Smooth Withe-rod
Viburnum nudum as to Ontario reports, not of L. = Viburnum cassinoides L. (Morton \& Venn 1990)

V. opulus L., Guelder-Rose, Snowball-tree

var. americanum Ait. Highbush Cranberry, Branberry-bush, Branberry-tree, Grouseberry, Squawbush, Pimbina

$=\mathrm{V}$. eradiatum (Oakes) House

$=\mathrm{V}$. trilobum Marsh.

*var. opulus, Snowball-tree, Guelder Rose

Viburnum pubescens (of authors) = Viburnum rafinesquianum Schultes

V. rafinesquianum Schultes, Downy Arrow-wood $=\mathrm{V}$. affine Bush ex Schneider $=\mathrm{V}$. affine var. hypomalacum Blake $=\mathrm{V}$. pubescens (of authors)

$=\mathrm{V}$. rafinesquianum var. affine (Bush ex Schneider) House

Viburnum recognitum Fern. $=$ Viburnum dentatum L. var. lucidum Ait.

Viburnum trilobum Marsh. = Viburnum opulus L. var. americanum Ait.

VICIA L. (Fabaceae)

V. americana Muhl. ex Willd., American Vetch, Purple Vetch, Tare

Vicia angustifolia L., not Reich. = Vicia sativa L. ssp. nigra (L.) Ehrh.

V. caroliniana Walt., Carolina Vetch, Wood-vetch $=\mathrm{V}$. parviflora Michx.

V. cracca L., Cow Vetch, Tufted-vetch, Canadapea

ssp. cracca

Vicia dasycarpa Tenore $=$ Vicia villosa Roth ssp. varia (Host) Corb.

Vicia parviflora Michx. = Vicia caroliniana Walt.

*V. sativa L., Common Vetch, Spring-vetch, Tare

*ssp. nigra (L.) Ehrh. Narrowleaf Vetch $=\mathrm{V}$. angustifolia L., not Reich.

$=$ V. sativa var. angustifolia (L.) Ehrh.

*ssp. sativa $=\mathrm{V}$. sativa var. linearis Lange

*V. tetrasperma (L.) Schreb., Four-seeded Vetch, Lentil-vetch, Sparrow-vetch, Slender Vetch

$=\mathrm{V}$. tenuissima (Bieb.) Schinz \& Thell. $=\mathrm{V}$. tetrasperma var. tenuissima Druce

Vicia tenuissima (Bieb.) Schinz \& Thell. = Vicia tetrasperma (L.) Schreb. 
*V. villosa Roth, Hairy Vetch, Wooly-pod Vetch

*ssp. varia (Host) Corb.

$=\mathrm{V}$. dasycarpa Tenore

*ssp. villosa

Vilfa vaginiflora Torr. ex Gray = Sporobolus vaginiflorus (Torr. ex A. Gray) Wood

VINCA L. (Apocynaceae)

*V. minor L., Periwinkle, Running Myrtle, Lesser Periwinkle

Vincetoxicum album (Mill.) Aschers. = Cynanchum vincetoxicum (L.) Pers.

Vincetoxicum hirundinaria Medik. $=$ Cynanchum vincetoxicum

Vincetoxicum nigrum (L.) Moench = Cynanchum nigrum (L.) Pers.

Vincetoxicum rossicum (Kleopov) Barb. = Cynanchum rossicum (Kleop.) Borh.

VIOLA L. (Violaceae)

V. adunca Sm., Western Dogfoot-violet, Hookspur-violet, Labrador-violet

$=\mathrm{V}$. adunca var. minor (Hooker) Fern.

$=\mathrm{V}$. huronensis Greene

$=\mathrm{V}$. labradorica Schrank

$=\mathrm{V}$. subvestita Greene

Viola $\times$ abundans House $=$ Viola sagittata Ait.

V. affinis LeConte, LeConte's Violet

$=\mathrm{V}$. latiuscula Greene

$=\mathrm{V}$. sororia $\mathrm{L}$. of authors in part

V. affinis $\times$ palmata $=$ V. slavinii House, Violet

$=\mathrm{V}$. davisii House

$=\mathrm{V} . \times$ discors House

V. affinis $\times$ sagittata var. ovata $=\mathrm{V} . \times$ hollickii House, Violet

*V. arvensis Murr., European Field-pansy, Wild Pansy

V. blanda Willd., Sweet White Violet

$=\mathrm{V}$. blanda var. palustriformis A. Gray

$=\mathrm{V}$. incognita Brainerd

$=\mathrm{V}$. incognita var. forbesii Brainerd

$=\mathrm{V}$. macloskeyi of authors in part, not Lloyd

Viola blanda of authors, not Willd. = Viola macloskeyi Lloyd ssp. pallens (Banks ex DC.) M. Baker

Viola brainerdii Greene $=$ Viola renifolia A. Gray

V. canadensis L., American Dog-violet, Tall White Violet, Canada Violet
$=\mathrm{V}$. canadensis var. corymbosa of NY

reports, not Nutt. ex Torr. \& A. Gray

$=\mathrm{V}$. canadensis var. rugulosa (Greene)

Hitchc.

$=\mathrm{V}$. rugulosa of $\mathrm{NY}$ reports, not Greene

Viola canina var. sylvestris (of authors) $=$ Viola sororia Willd.

V. conspersa Reichenb., American Dog-violet $=\mathrm{V}$. muhlengergii Torr.

Viola cucullata Ait. = Viola sororia Willd.

Viola davisii House $=[$ V . affinis $\times$ palmata $=\mathrm{V}$. slavinii House]

Viola $\times$ discors House $=[$ V. affinis $\times$ palmata $=$ V. slavinii House]

Viola domestica Pollard = Viola sororia Willd.

Viola emarginata (Nutt.) LeConte $=$ Viola sagittata Ait.

Viola eriocarpa Schwein. $=$ Viola pubescens Ait.

Viola eriocarpa var. leiocarpa Fern.\& Wieg. = Viola pubescens Ait.

Viola fimbriatula Sm. $=$ Viola sagittata Ait.

Viola huronensis Greene $=$ Viola adunca Sm .

Viola incognita Brainerd = Viola blanda Willd .

Viola incognita var. forbesii Brainerd = Viola blanda Willd.

Viola kitaibeliana var. rafinesquii (Greene) Fern. = Viola rafinesquii Greene

Viola labradorica Schrank = Viola adunca Sm.

V. lanceolata L., Lance-leaved Violet, Waterviolet

$=\mathrm{V}$. lanceolata ssp. vittata of NY report, not

(Greene) Weath. \& Griscom

Viola latiuscula Greene $=$ Viola affinis LeConte

Viola lewisiana DC. $=$ Viola striata Ait.

Viola lovelliana Brainerd = Viola palmata L .

V. macloskeyi Lloyd., Sweet White Violet

ssp. pallens (Banks ex DC.) M. Baker, Pale Violet

$=\mathrm{V}$. blanda of authors, not Willd.

= V. macloskeyi var. pallens (Banks ex DC.) Hitchc.

$=\mathrm{V}$. pallens (Banks ex DC.) Brainerd

Viola macloskeyi of authors in part, not Lloyd $=$

Viola blanda Willd.

Viola muhlengergii Torr. $=$ Viola conspersa

Reichenb.

V. nephrophylla Greene, Northern Bog-violet

Viola obliqua Hill = Viola sororia Willd.

Viola ochroleuca Schwein = Viola striata Ait. 
*V. odorata L., English Violet

Viola pallens (Banks) Brainerd = Viola macloskeyi

Lloyd ssp. pallens (Banks ex DC.) M.

Baker

V. palmata L., Palmate Blue Violet, Early Blue

Violet, Wood-violet, Wild Okra

$=\mathrm{V}$. lovelliana Brainerd, see Zander \&

Pierce, 1979, p. 4.

$=\mathrm{V}$. palmata var. triloba (Schwein.) Gingerns ex DC.

$=\mathrm{V}$. triloba Schwein.

Viola papilionacea Pursh = Viola sororia Willd.

Viola pectinata Bickn. $=$ Viola palmata $\mathrm{L}$.

V. pedata L., Bird's-foot Violet, Pansy-violet, Johnny-jump-up

$=\mathrm{V}$. pedata var. lineariloba DC.

Viola pensylvanica Michx. = Viola pubescens Ait.

Viola pensylvanica var. leiocarpa (Fern.\& Wieg.)

Fern. $=$ Viola pubescens Ait.

Viola priceana Pollard = Viola sororia Willd.

V. pubescens Ait., Smooth Yellow Violet

$=\mathrm{V}$. eriocarpa Schwein.

$=\mathrm{V}$. eriocarpa var. leiocarpa Fern.

$=\mathrm{V}$. pensylvanica Michx.

$=\mathrm{V}$. pensylvanica var. leiocarpa $($ Fern. $\&$

Wieg.) Fern.

$=\mathrm{V}$. pubescens var. eriocarpa (Schwein. $)$

Russell

$=\mathrm{V}$. pubescens var. leiocarpa (Fern. \&

Wieg.) Seym.

$=\mathrm{V}$. pubescens var. peckii House

$=\mathrm{V}$. pubescens var. scabriuscula Schwein. ex

Torr. \& A. Gray

(*?) V. rafinesquii Greene, American Field-pansy, Field-pansy

$=\mathrm{V}$. kitaibeliana var. rafinesquii (Greene)

Fern.

$=\mathrm{V}$. tenella Muhl.

V. renifolia A. Gray, Kidney-leaved Violet,

Northern White Violet

$=\mathrm{V}$. brainerdii Greene

$=\mathrm{V}$. renifolia var. brainerdii (Greene) Fern.

V. rostrata Pursh, Long-spurred Violet

V. rotundifolia Michx., Round-leaved Yellow

Violet, Early Yellow Violet.

V. sagittata Ait., Arrow-leaved Violet, Northern

Downy Violet, Fringed Violet

$=\mathrm{V} . \times$ abundans House

$=\mathrm{V}$. emarginata $($ Nutt. $)$ LeConte
$=\mathrm{V}$. fimbriatula $\mathrm{Sm}$

$=\mathrm{V}$. sagittata var. ovata (Nutt.) Torr. \& A. Gray

V. sagittata $\times$ sororia $=V$. conjugens Greene, Violet

$=\mathrm{V} . \times$ aberrans Greene ex Stone

$=\mathrm{V} . \times$ fernaldii House

$=\mathrm{V} . \times$ greenei House

$=\mathrm{V} . \times$ greenmanii House

$=$ Perhaps this is Viola sagittaria var. ovata $\times$ sororia $=$ Viola $\times$ parca House recorded for Erie Co. by NYFA July 2005.

V. selkirkii Pursh ex Goldie, Great-spurred Violet, Selkirk's Violet

V. septentrionalis Greene, Northern Blue Violet

V. sororia Willd., Wooly Blue Violet

$=\mathrm{V} . \times$ bissellii House

$=\mathrm{V}$. canina var. sylvestris (of authors)

$=\mathrm{V}$. cucullata Ait.

$=\mathrm{V}$. domestica Pollard

$=\mathrm{V}$. obliqua Hill

$=\mathrm{V}$. papilionacea Pursh

$=\mathrm{V}$. priceana Pollard

$=\mathrm{V}$. sylvestris Lam. of Gray's Manual 1887

Viola sororia L. of authors in part = Viola affinis LeConte

V. striata Ait., Creamy Violet, Pale Violet, Striped Violet, Cream Violet

$=\mathrm{V}$. lewisiana DC.

$=\mathrm{V}$. ochroleuca Schwein

Viola subvestita Greene = Viola adunca Sm.

Viola sylvestris Lam. of Gray's Manual $1887=$ Viola sororia Willd.

Viola tenella Muhl. $=$ Viola rafinesquii Greene

Viola triloba Schwein. = Viola palmata L.

Virea autumnalis (L.) S. Gray = Leontodon autumnalis L.

Virea autumnalis var. pratensis (L.) House $=$ Leontodon autumnalis $\mathrm{L}$.

Virea hispida (L.) S. Gray = Leontodon hispidus L.

Virea nudicaulis (L.) House $=$ Leontodon taraxacoides (Vill.) Merat

Virgulus ericoides $(\mathrm{L}$.$) Reveal =$ Aster ericoides L.

Virgulus novae-angliae (L.) Reveal \& Keener $=$ Aster novae-angliae L.

VITIS Adanson (Vitaceae)

V. aestivalis Michx., Summer Grape, Pigeongrape, Silverleaf-grape 
$=\mathrm{V}$. aestivalis var. argentifolia (Munson)

Fern.

$=\mathrm{V}$. argentifolia Munson

$=$ Vitis bicolor (of authors)

V. aestivalis $\times$ labrusca $=$ Vitis $\times$ labrusca Bailey

Vitis argentifolia Munson $=$ Vitis aestivalis Michx.

Vitis bicolor (of authors) = Vitis aestivalis Michx.

Vitis cordifolia (of authors) = Vitis riparia Michx.

V. labrusca L., Fox-grape, Skunk-grape

$=\mathrm{V}$. labrusca var. subedentata Fern.

$=\mathrm{V}$. labruscana Bailey

V. labrusca $\times$ riparia $=$ V. $\times$ novae-angliae Fern., New England Grape

V. riparia Michx., Frost-grape, Riverbank Grape, Dune-grape

$=\mathrm{V}$. cordifolia (of authors)

$=\mathrm{V}$. riparia var. syrticola (Fern. \& Wieg.)

Fern.

$=\mathrm{V}$. vulpina of old NY reports, not L.

Vitis vulpina (of authors) $=$ Vitis riparia Michx.

VITTARIA Sm. (Vittariaceae)

V. appalachiana Farrar \& Mickel, Appalachian

Shoestring-fern, Appalachian Vittaria

VULPIA K. Gmel. (Poaceae)

V. octoflora (Walt.) Rydb., Slender Fescue, Sixweeks-fescue, Six-week's-grass

var. glauca (Nutt.) Fern.

$=$ Festuca octoflora Walt.

$=$ F. tenella Willd.

= Vulpia octoflora var. tenella (Willd.) Fern.

WALDSTEINIA Willd. (Rosaceae)

W. fragarioides (Michx.) Tratt., Barren

Strawberry, False Strawberry

$=$ Dalibarda fragarioides Michx.

Warnera canadensis (L.) Mill. $=$ Hydrastis

canadensis $\mathrm{L}$.

WOLFFIA Horkel ex Schleid. (Lemnaceae)

Wolffia arrhiza (L.) Wimmer in Macdonald $1990=$ Wolffia columbiana Karst.

W. borealis (Engelm.) Landolt, Dotted

Watermeal, Watermeal $=$ L. punctata Griseb.

W. columbiana Karst., Watermeal

$=\mathrm{W}$. arrhiza (L.) Wimmer in Macdonald 1990
WOODWARDIA J. Smith (Blechnaceae)

W. virginica (L.) Sm., Virginia Chain-fern $=$ Anchistea virginica (L.) C. Presl

$=$ Blechnum virginicum $\mathrm{L}$.

XANTHIUM L. (Asteraceae)

*X. spinosum L., Spiny Cockle-bur

X. strumarium L., Cockle-bur var. canadense (Mill.) Torr. \& A. Gray

$=\mathrm{X}$. echinatum Murr.

$=\mathrm{X}$. italicum Moretti

$=\mathrm{X}$. ovaliforme Wallr.

$=\mathrm{X}$. pensylvanicum Wallr.

$=\mathrm{X}$. speciosum Kearney

$=X$. varians Greene

Xanthoxylum (orth. var.) = Zanthoxylum L.

XYRIS Gronov. ex L. (Xyridaceae)

Xyris caroliniana of authors, not Walt. $=$ Zyris difformis Chapm.

X. difformis Chapm., Carolina Yellow-eyed Grass, Yellow-eyed Grass

$=$ Xyris caroliniana of authors, not Walt.

YUCCA L. (Agavaceae)

*Y. filamentosa L. Yucca, Adam's-needle, Spanish Bayonette

$=$ Y. flaccida Haw

$=$ Y. smalliana Fern.

Yucca flaccida Haw = Yucca filamentosa L.

Yucca smalliana Fern. $=$ Yucca filamentosa L.

ZANNICHELLIA L. (Zannichelliaceae)

Z. palustris L., Horned Pondweed

$=$ Zannichellia palustris var. major (Boenn.)

Koch

ZANTHOXYLUM L. (Rutaceae)

Z. americanum Mill., Prickly-ash, Tooth-ache

Tree

ZEA L. (Poaceae)

*Z. mays L., Corn, Sweetcorn, Maize

ZIGADENUS Michx. (Liliaceae)

Z. elegans Pursh, White Camass, Alkali-grass

ssp. glaucus (Nutt.) Hultén

$=$ Z. glaucus (Nutt.) Nutt. 
$=\mathrm{Z}$. chloranthus (of authors)

ZIZANIA L. (Poaceae)

Z. aquatica L., Southern Wild Rice, Indian Rice

$=\mathrm{Z}$. palustris of early American authors, not L.

Zizania aquatica var. angustifolia Hitchc. = Zizania palustris L.

Zizania aquatica var. interior Fassett $=$ Zizania palustris L.

Z. palustris L., Northern Wild Rice

$=\mathrm{Z}$. aquatica of early American authors, not L.

$=$ Z. aquatica var angustifolia Hitchc.

$=\mathrm{Z}$. aquatica var. interior Fassett

ZIZIA Koch (Apiaceae)

Z. aptera (A. Gray) Fern., Heart-leaved Alexanders, Golden Alexanders

$=$ Thaspium trifoliatum (L.) A. Gray var. apterum A. Gray

= Zizia cordata (Walt.) Koch ex DC.

$=$ Z. cordata var. aptera A. Gray

Z. aurea (L.) Koch, Golden Alexanders

$=$ Thaspium aureum Nutt., according to Morton and Venn (1990), see discussion under Thaspium trifoliatum var. aureum.

Zizia cordata (Walt.) Koch ex DC. = Zizia aptera (Gray) Fern.

Zizia integerrima DC. $=$ Taenidia integerrima (L.) Drude

Zosterella see Heteranthera

Zosterella dubia (Jacq.) Small = Heteranthera dubia (Jacq.) MacM.

Zygadenus orth. var. Zigadenus 


\section{BIBLIOGRAPHY}

A bibliography is here included of texts used in augmenting the list and synonymy-which is a composite of the work of Zander \& Pierce (1979) who updated Zenkert's names in his original flora to the system of Gleason \& Cronquist (1963). Mitchell's New York state checklist of 1986 is followed here, as well as the 1997 version with Gordon Tucker. Certain nomenclatural additions derive from Morton \& Venn (1990). Extensive use has been made of the Contributions to a Flora of New York State, edited by Richard S. Mitchell, and to floristic publications by the Ontario Ministry of Natural Resources. Rare Plant Status lists edited by Steve Young periodically issued by the New York Natural Heritage Program in New York State have provided much precise data, as has the four-part publication of the Atlas of the Rare Vascular Plants of Ontario, edited by Argus, Pryer, White and Keddy from 1982 to 1987-the concentration of rare plants in the Niagara Peninsula being rather conspicuous there.

Argus, G. W., K. M. Pryer, D. J. White and C. J. Keddy, eds. 1982-87. Atlas of the rare vascular plants of Ontario. Four parts. National Museum of Natural Sciences, Ottawa. (looseleaf).

Bailey, L. H. 1949. Manual of Cultivated Plants. Macmillan Company, New York.

Ball, P. W. 1986. White water-buttercups (Ranunculus subgenus Batrachium) In Ontario. The Plant Press 4(3):78-80.

Bassett, I. J. and Munro, D. B. 1985. The biology of Canadian weeds 67. Solanum ptychanthum Dun., $S$. nigrum L. and S. sarrachoides Sendt. Canadian Journal of Plant Sciences 65: 401-414.

Boivin, Bernard. 1966. Naturaliste Canadien 93:590 [no title: cited by Voss, 1985 p.214 with regard to Ranunculus, subgenus Batrachium.]

Burgess, E. S. 1877. The Chautauqua Flora, a Catalogue of the Plants of Chautauqua Co., New York, Native or Naturalized; extending through the Cryptogamous Plants, to the End of the Hepaticae. Privately Printed, 37 pp., copy in Research Library of Buffalo Museum of Science (Botanical Pamphlets, Vol. 6). According to R. H. Zander (pers. comm.) the Burgess Herbarium cannot be located except for specimens of Aster at the New York Botanical Garden.

Catling, P. M., Reznicek, A. A., and Riley, J. L. 1977. Some new and interesting grass records from southern Ontario. Can. Field-Nat. 91:350-359.

Clemants, S E. 1990. Juncaceae (Rush Family) of New York State. New York State Museum Bull. 475, Albany.

Clemants, S E. 1992. Chenopodiaceae and Amaranthaceae of New York State. New York State Museum Bull. 485. Albany, New York.

Clinton, G. W. 1864. Plants of Buffalo and Its Vicinity. Buffalo, New York. Also printed in the Annual Report of the Regents on the State Cabinet (for 1863) 17: 24-35.

Cody, W. J. 1962. Sorbaria sorbifolia (L.) A.Br., False Spiraea, persisting and spreading after cultivation in Canada. Canadian Field-Naturalist 76:104-107.

Cook, C. D. K. 1966. A monographic study of Ranunculus subgenus Batrachium (DC.) A. Gray. Mitteilunger der Botanischen Staatssammlung München 6:47-237.

Cook, E. E. 1973. The vascular plants of Moss Lake Sanctuary, Allegany County, N.Y. Science Studies, St. Bonaventure Univ. 29:7-34, map.

Dalton, D. A. \& A. W. Naylor. 1975. Studies in nitrogen fixation by Alnus crispa. American Journal of Botany 62:76-80.

Daly, G. T. 1966. Nitrogen fixation by nodulated Alnus rugosa. Canadian Journal of Botany 44: 16071621.

Day, D. F. 1882. The plants of Buffalo and its vicinity: series 1, Phaenogamae. Bull. Buffalo Soc. Nat. Sci. 4:65-152, map. 
Day, David F. 1882. The Plants of Buffalo and Its Vicinity. Bull. Buffalo Soc. Nat. Sci., Vol. 4, April 1882, pp. 65-279, inclusive of the first supplement. Also reprinted as a pamphlet, 215 pages, Buffalo, N.Y., 1884.

Day, David F. 1886. The Plants of Buffalo and Its Vicinity. Second supplement, Bull. Buffalo Soc. Nat. Sci. Vol. 5(2), 1886, pp. 85-96.

Day, D. F. 1888. Catalogue of the Niagara Flora. A Catalogue of the Flowering and Fern-Like Plants Growing Without Cultivation in the Vicinity of the Falls of the Niagara. Annual Report of Commissioners of the State Reservation at Niagara for 1887, pp. 85-96. Also printed as a pamphlet, Troy, N.Y., 1888.

Dore, William G. \& J. McNeill. 1980. Grasses of Ontario. Research Branch Agriculture Canada Monograph 26.

Eaton, Steven W. \& Edith Feuerstein Schrot. 1987. A Flora of the Vascular Plants of Cattaraugus County, New York. Bull. of the Buffalo Soc. Nat. Sci. Vol. 31. Buffalo, New York.

Fernald, Merrit Lyndon. 1950. Gray's Manual of Botany. ed. 8. American Book Co., New York.

Gleason, Henry A. 1952. The New Britton and Brown Illustrated Flora of the Northeastern United States and Adjacent Canada. 3 Vols. New York Botanical Garden. New York.

Gleason, H. A. \& A. Cronquist. 1963. Manual of Vascular Plants of Northeastern United States and Adjacent Canada. Ed.1. New York Botanical Garden, Bronx, New York.

Gleason, H. A. \& A. Cronquist. 1991. Manual of Vascular Plants of Northeastern United States and Adjacent Canada. Ed. 2. New York Botanical Garden, Bronx, New York.

Gould, J. 1989. A Biological Inventory and Evaluation of Short Hills Provincial Park. Ontario Ministry of Natural Resources, Parks and Recreational Areas Section Central Region, Richmond Hill, Ontario

Harris, Ben Charles. 1968. Eat the Weeds. Barre Publishers. Barre, Mass.

House, Homer H. 1924. Annotated list of the Ferns and Flowering Plants of New York State. New York State Museum Bulletin 254, Albany, New York. The treatment of Crataegus in this publication was by W. W. Eggleston.

Isely, D. 1983. The Desmodium paniculatum (L.) DC. (Fabaceae) complex revisited. Sida 10(2): 142-158.

Jones, Almut G. 1989. Aster and Brachyactis in Illinois. Illinois Natural History Survey Bulletin Vol. 34 (2):138-194).

Kato, Masahiro. 1933. "Athyrium" in Flora of North America Vol. 2: Pteridophytes and Gymnosperms. Oxford University Press. New York.

Lamere, Clifford W. 1991. Sky-blue Aster at Niagara River Gorge. NYFA Newsletter Vol.2(3):2. New York Flora Association of the New York State Museum Institute.

Lamere, Clifford W. 1991. Seaside Goldenrod (Solidago sempervirens L.) at Niagara Falls. NYFA Newsletter Vol. 2(3):4. New York Flora Association of the New York State Museum Institute.

Larson, B.M.H. 1995. An assortment of new county plant records for southern Ontario: evidence for Murphy's Law. Field Botanists of Ontario Newsletter 8(1):9-15.

Mabberley, D. J. 1987. The Plant-Book. A portable dictionary of the higher plants. Cambridge University Press, New York.

Macdonald, Ian D. 1990. A Biological Inventory and Evaluation of the Point Abino Peninsula Area of Natural and Scientific Interest. Ontario Ministry of Natural Resources, Parks and Recreational Areas Section, Central Region, Aurora, Ontario.

Macoun, John. 1902. Catalogue of Canadian Plants. Geological Survey of Canada. Parts 1-6, Montreal, 1883-1892; part 7, Ottawa 1902.

McIntosh, Karen L. \& Paul M. Catling. 1979. Notes on the flora of the Canadian portion of the Niagara Frontier. Ontario Field Biologist 33:1-11.

Mitchell, Richard S. 1988. Platanaceae through Myricaceae of New York State. New York State Museum Bulletin 464. State Education Department, Albany, New York. 
Mitchell, Richard S. \& J. Kenneth Dean. 1978. Polygonaceae (Buckwheat Family) of New York State. New York State Museum Bulletin 431.

Mitchell, Richard S. 1984. Atlas of New York State Ferns. New York State Museum Bulletin 456.

Mitchell, Richard S. 1986. A Checklist of New York State Plants. New York State Museum Bulletin 458.

Mitchell, Richard S \& J. Kenneth Dean. 1982. Ranunculaceae (Crowfoot Family) of New York State. New York State Museum Bulletin 446.

Mitchell, Richard S. \& Gordon C. Tucker. 1997. Revised Checklist of New York State Plants. New York State Museum Bulletin 490.

Mohlenbrock, R. H. 1990. The Illustrated Flora of Illinois. Flowering plants, nightshades to mistletoe. Southern Illinois University Press, Carbondale and Edwardsville. 224 pp.

Morton, J. K. 1979. Observations on Houghton's goldenrod (Solidago houghtonii T. \& G.). Michigan Botanist 18:31-35.

Morton, J. K. \& Joan M. Venn. 1984. The Flora of Manitoulin Island. Publ. University Waterloo Biology Series 28: $181 \mathrm{pp}$.

Morton, J. K. \& Joan M. Venn. 1990. A Checklist of the Flora of Ontario Vascular Plants. University of Waterloo Biology Series 34. Obtainable from Department of Biology, University of Waterloo, Waterloo, Ontario, Canada, N2L 3G1.

Mosyakin, S. L. 1995. New taxa of Corispermum L. (Chenopodiaceae), with preliminary comments on the taxonomy of the genus in North America. Novon 5(4): 340-353.

Muenscher, W. C. 1946. The vegetation of Bergen Swamp. I. The vascular plants. Proc. Rochester Academy of Science 9:64-117.

New York Flora Association. 1990. Preliminary Vouchered Atlas of New York State Flora. Ed. 1. New York State Museum Intsitute, Albany. NYFA: New York Flora Association.

Oldham, M. J. 1988. Tall Thoroughwort (Eupatorium altissimum L.) in Ontario. The Plant Press 5(1):1619.

Oldham, M. J. 1999. Natural Heritage Resources of Ontario: Rare Vascular Plants. Third Edition. Peterborough, Ont.: Natural Heritage Information Centre, Ontario Ministry of Natural Resources.

Peterson, Lee. 1978. A Field Guide to Edible Wild Plants of Eastern and Central North America. Houghton Mifflin Co., Boston.

Peterson, Roger Tory and Margaret McKenny. 1968. A Field Guide to Wildflowers of Northeastern and North-central North America. Houghton Mifflin Co., Boston.

Phipps, J. B. 1988. Typification of Crataegus crus-galli L. (Rosaceae) and nomenclatural notes on some related species of hawthorn. Botanical Journal of the Linnean Society 96(4): 359-369.

Reznicek, A. \& P. M. Catling. 1984. Notes on Canadian sedges, Cyperaceae. Canadian Field Naturalist 98(2): 209-214.

Sargent, C. S. 1913. "Crataegus in New York” in New York State Museum Bulletin 167:53-124.

Schuyler, A. E. 1974. Typification and application of the names Scirpus americanus Pers., S. olneyi Gray and S. pungens Vahl. Rhodora 76:51-52.

Semple, John C. and Stephen B. Heard. 1987. The Asters of Ontario: Aster L. and Virgulus Raf. (Compositae: Astereae). University of Waterloo Biology Series 30.

Semple, John C. \& Gordon S. Ringius. 1983. Goldenrods of Ontario: Solidago and Euthamia. University of Waterloo Biology Series 26.

Smith, Stanley J. 1965. Checklist of the grasses of New York State. New York State Museum Bulletin 403:1-44.

Stone, Donald E. 1997. Carya, in Flora of North America, Oxford University Press, New York. pp. 417425

Stuckey, Ronald L. 1968. Distributional History of Butomus umbellatus (Flowering-rush) in the Western Lake Erie and Lake St. Clair Region. Michigan Botanist 7:134-142. 
Uphof, J. C. Th. 1968. Ed 2. Dictionary of Economic Plants. Verlag von J. Cramer, Stechert-Hafner Service Agency, Inc., New York, N.Y.

Voss, Edward G. Michigan Flora. 1972. Part I. Gymnosperms and Monocots. Cranbrook Institute of Science Bulletin 55 and University of Michigan Herbarium, Ann Arbor.

Voss, Edward G. Michigan Flora. 1985. Part II. Dicots (Saururaceae-Cornaceae). Cranbrook Institute of Science Bulletin 59 and University of Michigan Herbarium, Ann Arbor.

Voss, Edward G. Michigan Flora. 1996. Part III. Dicots (Pyrolaceae-Compositae). Cranbrook Institute of Science Bulletin 61 and University of Michigan Herbarium, Ann Arbor.

Wagner, Warren H, Robbin C. Moran \& Charles R. Werth 1993. Flora of North America Vol. 2: Pteridophytes and Gymnosperms. Oxford University Press. New York.

Wagner, Warren H. Jr. \& Joseph M. Beitel. 1993. "Lycopodiaceae" in Flora of North America Vol. 2: Pteridophytes and Gymnosperms. Oxford University Press. New York.

Wagnon, H. K. 1952. A revision of the genus Bromus, section Bromopsis, of North America. Brittonia 7:415-480.

Young, Steven M., 1998, New York Natural Heritage Program Rare Plant Status List. New York Natural Heritage Program. Latham, New York.

Zander, Richard H. \& Gary J. Pierce. 1979. Flora of the Niagara Frontier Region. Second Supplement and Checklist. Bull. Buffalo Soc. of Nat. Sci. Vol. 16. Buffalo, New York.

Zenkert, Charles A. 1934. Flora of the Niagara Frontier Region. Bull. of the Buff. Soc. of Nat. Sci. Vol. 16. Buffalo, New York. "Recently collected material" of Crataegus was determined by K. M. Wiegand (Cornell University. p. 175).

Zenkert, C. A. \& R. H. Zander. 1975. Flora of the Niagara Frontier Region, Supplement. Vol. 16. Bull. of the Buffalo Soc. of Nat. Sci., Buffalo, N.Y. 


\section{APPENDIX 1. NAMES NEW TO THE FLORA SINCE THE SECOND SUPPLEMENT (1979)}

There are 295 newly accepted names to the Flora, two of them doubtful. Of these, 12 are subspecies, 30 are varieties, 30 are hybrids; also 135 of the 288 are introductions. The New York Flora Association provided 101 names to the Niagara Frontier region from their online plant atlas, and these are marked "NYFA." A few names are reported elsewhere in the literature but the majority are deposited in the Clinton Herbarium of the Buffalo Museum of Science (BUF).

*Acer campestre L., Hedge-maple

*A. pseudoplatanus L., Sycamore-maple

A. rubrum $\times$ saccharinum $=$ A. $\times$ freemanii Murr., Soft Maple, Hybrid Swamp Maple

Actaea pachypoda $\times$ spicata $\mathrm{ssp}$. rubra, Baneberry

*Aegilops cylindrica Host, Jointed Goatgrass NYFA

*Aesculus glabra Willd., Ohio Buckeye

*Aethusa cynapium L., Fool's Parsley NYFA

Agrimonia rostellata Wallr., Agrimony

Agrostis perennans (Walt.) Tuckerm., Upland Bent, Autumn Bent NYFA

A. scabra Willd., Hairgrass, Fly-away Grass, Ticklegrass NYFA

Alisma gramineum Lej., Water-plantain, Grassyleaved Water-plantain

Allium burdickii (Hanes) A. Jones, Wild Leek

*A. cepa L., Garden Onion

*Alopecurus geniculatus L., Marsh-foxtail, Waterfoxtail

*Alopecurus pratensis L., Meadow Foxtail

*Althaea officinalis L., Marsh Mallow, White Mallow

*Amaranthus hypochondriacus L., Prince's Feather

*A. powellii S. Wats., Amaranth NYFA

*A. rudis Sauer, Water-hemp

*A. tuberculatus (Moq. ex DC.) Sauer, Rough-fruited Water-hemp, Tall Water-hemp

Amelanchier stolonifera Wieg., Serviceberry

*Amorpha fruiticosa L. False Indigo

*Ampelopsis brevipedunculata (Maxim.) Trautv., Porcelain Berry

*Athemis tinctoria L., Yellow Chamomile, Dyer's Chamomile

*Anthriscus cerefolium (L.) Hoffm., Chervil

*A. sylvestris (L.) Hoffm., Wild Chervil

*Arabis caucasica Schlect. ex Willd., Wall Rock-cress, Arrowleaf

A. drummondii $\times$ holboellii $=$ A. $\times$ divaricarpa $A$. Nels., Purple Rock-cress

*Aristida dichotoma L., Poverty Grass; Three-awn

*A. oligantha Michx., Prairie Three-awn NYFA

Aster dumosus L., Bushy Aster, Rice-button Aster
A. ericoides $\times$ novae-angliae $=$ A. $\times$ amethystinus Nutt., Amethyst Wreath Aster

A. nemoralis Ait., Bog Aster NYFA

A. ontarionis Wieg., Ontario Aster

A. puniceus var. firmus (Nees) Torr. \& A. Gray, Cornel-leaf Aster

A. schreberi Nees, Large-leaf Aster, Schreber's Aster

*Atriplex prostrata Boucher ex DC. in Lam. \& DC., Orach, Spearscale

*A. rosea L., Red Orach, Rosy Orach, Tumble-weed, Tumbling Orach

*Avena fatua ssp. fatua, Wild Oats

Baptisia lactea (Raf.) Thieret, Prairie False-Indigo

*Barbarea verna (Mill.) Aschers, Early Wintercress, Winter Belle-Isle, Land-Cress

*Betula pubescens Ehrh., Downy Birch NYFA

B. nigra L., River Birch, Red Birch

Brachyelytrum septentrionale (Babel) G. Tucker, Northern Shorthusk NYFA

*Brassica oleracea L., Cabbage, Kale, Broccoli, Cauliflower, Kohlrabi, Brussels Sprouts

*B. rapa L., Field Mustard, Turnip, Bok Choy, Chinese Cabbage

*Brunnera macrophylla (Adams) I. M. Johnston, Siberian Bugloss, Garden Forget-me-not

*Buddleja davidii Franch., Summer Lilac, Butterflybush, Buddleja NYFA

Bulbostylis capillaris (L.) Clarke, Sand-rush NYFA

*Cardamine flexuosa With., Rock-cress

Carex annectens (Bickn.) Bickn.

var. annectens

var. xanthocarpa (Bickn.) Wieg.

C. appalachica Webber \& Ball NYFA

C. emoryi Dewey ex Torrey, Emory's Sedge

C. flaccosperma Dewey, Sedge

C. formosa Dewey, Handsome Sedge NYFA

C. gracilescens Steud.

[C. haydenii Dewey, Hayden's Sedge doubtful report]

[C. hyalinolepis Steud., Sedge doubtful report]

C. meadii Dewey, Mead's Sedge

C. merritt-fernaldii Mackz., Fernald's Sedge NYFA

C. seorsa Howe ex Gord. \& Howe, Sedge NYFA 
C. sprengelii Dewey ex Sprengel

C. tonsa (Fern.) Bickn. var. rugosperma (Mackenzie) Crins, Umbelliform Sedge NYFA

Catalpa speciosa (Warder ex Barney) Engelm.: Catalpa, Indian-bean, Catawba, Cigar-tree

Celastrus orbiculata Thunb., Oriental Bittersweet

Chaerophyllum procumbens (L.) Crantz., Spreading Chervil NYFA

*Chenopodium album var. missouriense (Aellen) Bassett \& Crompton, Missouri Goose-foot

*C. bonus-henricus L., Good-King-Henry

*C. murale L., Sowbane, Nettle-leaf Goosefoot

*C. rubrum L., Red Pigweed, Red Goosefoot, Coatblite

C. strictum var. glaucophyllum (Aellen) Wahl, Goosefoot

*Chionanthus virginicus L. Old-man's Beard, Fringe tree

Citrullus colocynthis (L.) Shrad. ex Eckl. \& Zeyh. Watermelon

*Clematis terniflora DC., Yam-leaf Clematis, Yamleaf Virgin's-bower

*Commelina communis var. ludens (Miq.) Pennell NYFA

*Coriandrum sativum L., Coriander NYFA

*Corispermum nitidum Kitaibel ex Schultes

*C. orientale Lamarck

Cornus foemina * ${ }^{\mathrm{ssp}}$. microcarpa (Nash) J. S. Wilson

*Coronopus didymus (L.) Sm., Wart-cress, Wartgrass, Lesser Swinecress NYFA

*Corylus avellana L., European Filbert, European Hazel NYFA

*Cosmos bipinnatus Cav., Cosmos NYFA

*Cotinus coggygria Scop., Smoke-tree

Crataegus jesupii Sarg., Hawthorn

*Crepis tectorum L., Hawk's-beard

*Crypsis schoenoides (L.) Lam., Crypsis, Heleochloa, Swamp Timothy

*Cucumis sativus L., Garden Cucumber

*Cucurbita pepo L., Pumpkin, Acorn Squash, Summer Squash NYFA

*Cynanchum rossicum (Kleop.) Borh. Swallow-wort NYFA

* Cynodon dactylon (L.) Pers., Bermuda-grass NYFA

Cyperus flavescens L. Galingale, Yellow Cyperus NYFA

*C. fuscus L., Dark Galingale, Flatsedge

Cystopteris protrusa (Weath.) Blasd., Lowland Fragile Fern NYFA

C. tenuis (Michx.) Desv., Fragile Fern NYFA

Desmodium glabellum (Michx.) DC., Dillen's Ticktrefoil, Tall Tick-clover or Tick-trefoil

D. perplexum Schub., Tick-clover, Tick-trefoil NYFA

*Dianthus deltoides L., Maiden-pink, Meadow-pink
*Dioscorea batatas Dcne., Yam, Chinese Yam, Cinnamon-vine NYFA

*Dipsacus laciniatus L., Cut-leaf Teasel

Draba reptans (Lambert) Fernald, Whitlow-grass

Dracocephalum parviflorum Nutt., Dragonhead

Dryopteris carthusiana $\times$ cristata $=\mathrm{D} . \times$ uliginosa (A. Br. ex Dowell) Druce, Wood-fern

D. carthusiana $\times$ intermedia $=$ D. $\times$ triploidea Wherry, Wood-fern

D. celsa (Palmer) Knowlt., Palmer \& Poll., Log-fern NYFA

D. celsa $\times$ goldiana, Log-fern NYFA

D. clintoniana $\times$ goldiana Wood-fern

D. cristata $\times$ intermedia $=$ D. $\times$ boottii $($ Tuckerm. $)$ Underw. NYFA

D. goldiana $\times$ marginalis $=\mathrm{D} . \times$ neowherryi $\mathrm{W}$. Wagner, Wood-fern

Echinochloa walteri (Pursh) Heller (not Nash ex Britt.), Water-millet, Walter's Cockspur-grass

*Echinops ritro ssp. ruthenicus (Bieb.) Nyman, Globe Thistle

*E. sphaerocephalus L., Globe Thistle

Eleocharis flavescens (Poir.) Urban., Olive Spikerush

Enemion biternatum Raf., False Rue-Anemone NYFA

*Epilobium parviflorum Schreber, Small-flowered Willow-herb

Equisetum hyemale $\times$ variegatum $=\mathrm{E} . \times$ mackii (Newm.) Brich., Rough-tooth Scouring-rush, Hybrid Scouring-rush

Eragrostis capillaris (L.) Nees, Lacegrass

E. spectabilis (Pursh) Steud., Purple Lovegrass, Tumblegrass

*Eriochloa acuminata (J. Presl) Kunth, Cup-grass

*Euonymus fortunei var. radicans (Sieb. ex Miq.) Rehd.

Eupatorium altissimum L., Tall Thoroughwort

E. fistulosum Barratt, Joe-Pye-weed, Trumpetweed

*Euphrasia stricta Wolff ex Lehm., Eyebright NYFA

*Fraxinus excelsior L., European Ash

*Froelichia gracilis (Hooker) Moq., Cottonweed, Slender Snake-cotton NYFA

Fuirena pumila (Torr.) Spreng., Umbrella-grass

* Gaillardia aristata Pursh, Blanket-flower

Galega officinalis L., Catgut, Goat's-rue

Galium circaezans var. hypomalacum Fern.

Gentiana linearis Froel., Narrow-leaved Gentian NYFA

Geum laciniatum Murray, Rough Avens, Herb-bennet

*G. urbanum L., Town-avens

G. verum (Raf.) Torrey \& A. Gray, Spring-avens

Gnaphalium purpureum L., Purple or Sweet Everlasting, Cudweed

*Gypsophila elegans Bieb., Showy Baby's Breath NYFA 
*Hedera helix L., English Ivy

*Helianthus pauciflorus ssp. subrhomboideus $\times$ tuberosus $=\mathbf{H} . \times$ laetiflorus Pers., Sunflower NYFA

*Hieracium flagellare Willd., Hawkweed

H. gronovii $\times$ venosum $=\mathbf{H} . \times$ marianum Willd., Hybrid Hawkweed NYFA

*Hordeum vulgare L., Barley, Pearl-barley NYFA

Hydrocotyle ranunculoides L.f., Floating Pennywort

H. verticillata var. fetherstoniana (Jennings) Mathias NYFA

Hypericum hypericoides ssp. multicaule (Michx. ex Willd.) Robson

H. prolificum L., Shrubby St. John's-wort

*Impatiens balsamina L., Balsam, Garden-balsam NYFA

Iris virginica var. schrevii (Small) Anders.

Isoetes engelmannii $\mathrm{A}$. $\mathrm{Br}$.

I. lacustris $\times$ tuckermanii $=I . \times$ heterospora A. Eaton

Juncus effusus var. conglomeratus (L.) Engelm. ex A. Gray

J. effusus var. pylaei (LaHarpe) Fern. \& Wieg. NYFA

*Lactuca saligna L., Willow-leaf Lettuce

*Larix decidua Mill., European Larch NYFA

*Lathyrus sylvestris L., Perennial or Everlasting Pea

Lespedeza hirta $\times$ intermedia $=$ L. $\times$ nuttallii Darl. NYFA

L. stuevii $\times$ virginica $=$ L. $\times$ neglecta Mackz. \& Bush NYFA

L. violacea (L.) Pers., Bush-clover

*Liatris aspera Michx., Rough Blazing-star

*L. spicata (L.) Willd., Blazing-star

*Ligustrum obtusifolium Sieb. \& Zucc., Privet

Lilium michiganense Farw., Michigan Lily

*Linaria dalmatica (L.) Mill., Dalmatian Toadflax

Linum medium var. texanum (Planch.) Fern., Southern Yellow Flax NYFA

Littorella uniflora (L.) Aschers., Shore-grass, Littorella

*Lonicera japonica Thunb., Japanese Honeysuckle, Gold-and-Silver-flower

*L. maackii (Rupr.) Maxim., Honeysuckle

*L. morrowii $\times$ tatarica $=$ L. $\times$ bella Zabel, Flyhoneysuckle

*L. xylosteum L., European Fly-honeysuckle

Ludwigia polycarpa Short \& Peter, Many-fruited False Loosestrife

Luzula campestris var. echinata (Small) Fern. \& Wieg.

L. campestris var. pallescens (Wahl.) Wahl., Common Wood-rush NYFA

*Lycopersicon esculentum Mill., Garden Tomato

Lycopodium annotinum var. pungens (La Pyl.) Desv. NYFA
L. digitatum $\times$ tristachyum $=\mathrm{L} \cdot \times$ habereri House NYFA

Lycopus rubellus Moench, Gypsy-wort, Waterhorehound

Lyonia ligustrina (L.) DC., Maleberry, HeHuckleberry, Male Blueberry NYFA

*Mahonia aquifolium (Pursh) Nutt., Oregon Grape, Grape-holly NYFA

Malus glaucescens Rehd., American Crab, Wild Crabapple NYFA

* Marsillia quadrifolia L., Water-clover, Pepperwort

*Matricaria recutita L., Wild Chamomile, False Sweet Chamomile, German Mayweed NYFA

*Mentha arvensis $\times$ spicata $=$ M. $\times$ gracilis Sole, Red Mint, Scotch Mint, Spotted Mint, Creeping Whorled Mint

Mikania scandens (L.) Willd., Climbing Boneset

* Miscanthus sinensis Anderss., Eulalia

*Muhlenbergia asperifolia (Nees \& Meyer) Parodi

*Myosotis stricta Link ex R. \& S., Forget-me-not

Panicum boreale Nash, Northern Panic-grass NYFA

P. villosissimum Nash, Panic Grass

Paronychia fastigiata (Raf.) Fern., Forked Chickweed

Parthenium integrifolium L., Wild Quinine

*Parthenocissus tricuspidata (Sieb. \& Zucc.) Planch. ex DC., Boston Ivy, Japanese Ivy, Cottage-ivy

*Penstemon calycosus Small, Penstemon, Beardtongue NYFA

*Philadelphus coronarius L., Garden Syringa, European Mock-orange

Physalis virginiana Mills., Virginia Ground-cherry NYFA

*Pisum sativum L., Common, Garden-, Green or Fieldpea NYFA

Plantago cordata Lam., Heart-leaved Plantain

Platanthera leucophaea (Nutt.) Lindl., Eastern Prairie Fringed Orchid

P. macrophylla (Goldie) P. M. Brown, Large Roundleaf Orchid, Pad-leaf

Poa nemoralis L., Wood Bluegrass

* Polygonum aviculare ssp. depressum (Meisner) Arcangeli NYFA

P. buxiforme Small, Knotweed, Knotgrass NYFA

*P. cespitosum var. longisetum (DeBruyn) Stewart, Low Smartweed

P. punctatum var. confertiflorum (Meisn.) Fassett

*P. ramosissimum Michx. var. ramosissimum NYFA

Polypodium appalachianum Haufler \& Windham, Appalachian Rockcap Fern. NYFA

Potamogeton gramineus $\times$ illinoensis $=\mathbf{P} . \times$ spathuliformis (Robbins) Morong, Pondweed

P. pusillus var. tenuissimus Mert. \& Koch NYFA

Proserpinaca palustris var. crebra Fern. \& Grisc. 
*Puccinellia distans *ssp. borealis (Holmb.) W. E. Hughes

*P. distans (Jacq.) Parl. ssp. distans

Pycnanthemum verticillatum (Michx.) Pers. var. verticillatum Mountain-mint

Quercus alba $\times$ montana $=$ Q. $\times$ saulii, Oak

Q. coccinea Muenchh., Scarlet Oak

Q. ilicifolia Wang.; Scrub-oak, Bear-oak NYFA

Q. shumardii Buckl., Shumard Oak

Ranunculus abortivus var. eucyclus Fern.

*R. ficaria L., Lesser Celandine

R. hispidus var. nitidus (Muhl. ex Ell.) Duncan, Swamp-buttercup NYFA

*Reseda lutea L., Yellow Mignonette, Scrambling Rocket NYFA

Rhododendron viscosum (L.) Torrey, Swamp Azalea NYFA

*Rhodotypos scandens (Thunb.) Makino, Jetbead, White Kerria

Rhus copallinum L., Shining Sumac, Winged Sumac, Dwarf Sumac NYFA

Rosa acicularis $\times$ blanda $=$ R. $\times$ housei Erlanson, House's Rose NYFA

*R. cinnamomea L., Damask Rose

*Rubus bifrons Vest ex Tratt., Himalaya-berry

*R. idaeus L. ssp. idaeus, European Red Raspberry

*R. phoenicolasius Maxim., Wineberry

Rumex maritimus var. fueginus (Phil.) Dusén NYFA

Sagittaria graminea Michx., Grass-leaf Sagittaria NYFA

Salix babylonica $\times$ fragilis $=\mathrm{S} . \times$ pendulina Wenderoth, Wisconsin Weeping Willow NYFA

S. cordata Michx., Sand-dune Willow, Heartleafwillow

*S. matsudana Koidz., Dragon-claw Willow, Corkscrew-willow

S. myricoides Muhl.

*S. pentandra L., Bay-leaf Willow NYFA

*Scilla siberica Haw. ex Andr., Scilla, Squill

Scirpus georgianus Harper, Bulrush

S. hudsonianus (Michx.) Fern., Alpine Bulrush, Alpine Cottongrass NYFA

S. pungens Vahl, Bulrush NYFA

S. smithii Gray, Bulrush

Scleria reticularis Michx., Nutrush, Whipgrass

Scutellaria incana Biehl., Hoary Skullcap

* Secale cereale L., Rye NYFA

*Sedum sarmentosum Bunge, Sedum

Senecio aureus $\times$ pauperculus, Groundsel NYFA

*S. viscosus L., Sticky Groundsel NYFA

*Setaria faberi Herrm., Giant Foxtail

*Silene cserei Baumg., Campion, Balkan Catchfly NYFA
*Sisymbrium loeselii L., Tall Hedge-mustard NYFA

Solanum ptychanthum Dunal, Black Nightshade, Poisonberry NYFA

Solidago odora Ait., Sweet Goldenrod

S. sempervirens L., Seaside-goldenrod

S. uliginosa var. linoides (Torrey \& A. Gray) Fern. NYFA

*Sorbaria sorbifolia (L.) A. Br., False Spiraea

*Sorghum bicolor (L.) Moench, Sorghum, Broomcorn NYFA

Sparganium androcladum (Engelm.) Morong, Burreed

*Spergularia rubra (L.) J. \& C. Presl, Common Sandspurry, Purple Sand-spurry, Sandwort

*S. salina J. \& C. Presl, Saltmarsh Sand-spurry NYFA

Sphenopholis pensylvanica (L.) Hitchc., Swamp-oats

Spiranthes casei Catl. \& Cruise, Lady's-tresses NYFA

S. ochroleuca (Rydb. ex Britt.) Rydb., Creamy Lady'sTresses

*Succisella inflexa (Kluk) Beck, Devil's-bit

*Syringa vulgaris L., Lilac, Purple Lilac, White Lilac

*Taxodium distichum (L.) Rich., Bald Cypress

*Tilia cordata Mill., Small-leaved Linden, European Linden, Heart-leaf Linden

Torreyochloa pallida var. fernaldii (Hitchc.) Church NYFA

Toxicodendron rydbergii (Small ex Rydb.) Greene, Giant Poison-ivy, Poison-oak, Creeping Poison-ivy

*Tradescantia virginiana L., Spiderwort, Widow's Tears NYFA

*Tragopogon dubius Scop., Goat's Beard

$* \mathbf{T}$. porrifolius $\times$ pratensis $=\mathbf{T}$. neohybridus Farw., Goat's-beard

Triadenum fraseri (Spach) Gleason, Marsh St. John's Wort, Pink-flowered St. John's-wort

*Trifolium campestre Schreber, Hop-clover, Low Hop-clover, Shamrock, Cow-clover NYFA

Trillium flexipes Raf., Nodding Trillium

Tripsacum dactyloides (L.) L., Gamma-grass

*Triticum aestivum L., Wheat NYFA

*Urtica urens L., Burning Nettle, Dog-nettle NYFA

Vaccinium boreale Hall \& Hald., High-mountain Blueberry NYFA

Verbena urticifolia var. leiocarpa Perry \& Fern. NYFA

*Verbesina alternifolia (L.) Britt., WingstemNYFA

*Veronica hederifolia L., Ivy-leaf Speedwell NYFA

V. peregrina ssp. xalapensis (Kunth) St. John \& Warren

V. scutellata L., Marsh-speedwell NYFA

*V. serpyllifolia ssp. humifusa (Dicks. ex With.) Syme, Thyme-leaved Speedwell 
Viburnum lantanoides Michx., Hobblebush, Witchhobble, Moosewood, Tanglewood

Viola adunca Sm., Western Dogfoot-violet, Hookspurviolet, Labrador-violet

V. affinis $\times$ palmata $=$ V. slavinii House, Violet NYFA

V. affinis $\times$ sagittata var. ovata $=\mathrm{V} . \times$ hollickii House, Violet NYFA

V. pedata L., Bird's-foot Violet, Pansy-violet, Johnnyjump-up NYFA

V. sagittata $\times$ sororia $=$ V . conjugens Greene, Violet NYFA
Vitis aestivalis $\times$ labrusca $=$ Vitis $\times$ labrusca Bailey NYFA

V. labrusca $\times$ riparia $=$ V. $\times$ novae-angliae Fern., England Grape NYFA

Vittaria appalachiana Farrar \& Mickel, Appalachian Shoestring-fern, Appalachian Vittaria

*Yucca filamentosa L. Yucca, Adam's-needle, Spanish Bayonette

*Zea mays L., Corn, Sweetcorn, Maize NYFA 


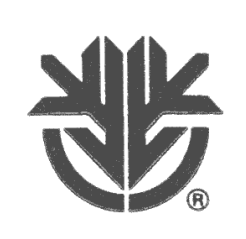

\section{MISSOURI BOTANICAL GARDEN}

\section{BIOGRAPHY}

Patricia Maureen Eckel was born in Niagara Falls, New York, and graduated from the State University of New York at Buffalo with B.A. Cum Laude in Greek and Latin Classics, 1981, B.A. Cum Laude in Art History, 1981, and M.A., Biology, 1984. She has contributed to the fields of plant taxonomy, botanical art, history of science, and botanical Latin.

Appointments include Research Scientist, Missouri Botanical Garden, 2002 to present; Research Associate, Division of Botany, Buffalo Museum of Science, 1979-1985 and 2003 to present, and Research Fellow, 1986-2002; Director of Moss Exchange, American Bryological and Lichenological Society, 1981-1992; Niagara Frontier Botanical Society founding member, Treasurer, 1983-1985, Secretary 1988-1990; Fellow (National), Explorer's Club, 1988 to present; Editor of the Clinton Papers, archives of the Research Library, Buffalo Society of Natural Sciences, 1990 to present; scientific illustrator, Flora of North America, Volumes 27-29 (bryophytes), 2000 to present, funded by National Science Foundation and Chanticleer Botanical Garden; collaborator, Department of Botany, Smithsonian Institution, 1985-1988.

She has published more than 75 scientific articles on both vascular plants and bryophytes since 1980. Her research focus is on the flora of the Niagara Falls region and the moss family Pottiaceae, including taxonomic treatments in the Flora of North America. Many of her contributions relevant to the botany and botanical history of western New York State and adjacent Ontario are online at two Missouri Botanical Garden Web sites:

http://www.mobot.org/plantscience/resbot/ and

http://www.mobot.org/plantscience/bfna/bfnamenu.htm.

Beginning in 1986, Ms Eckel published 27 articles on regional floristic botany published in Clintonia, the journal of the Niagara Frontier Botanical Society.

Ms. Eckel is presently a resident of St. Louis, Missouri, working at the Missouri Botanical Garden. 